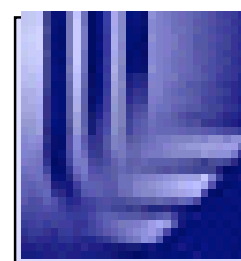

LAWRENCE LIVERMORE NATIONAL LABORATORY

\section{VISTA - A Vehicle for Interplanetary Space Transport Application Powered by Inertial Confinement Fusion}

\author{
C. D. Orth
}

May 16, 2003

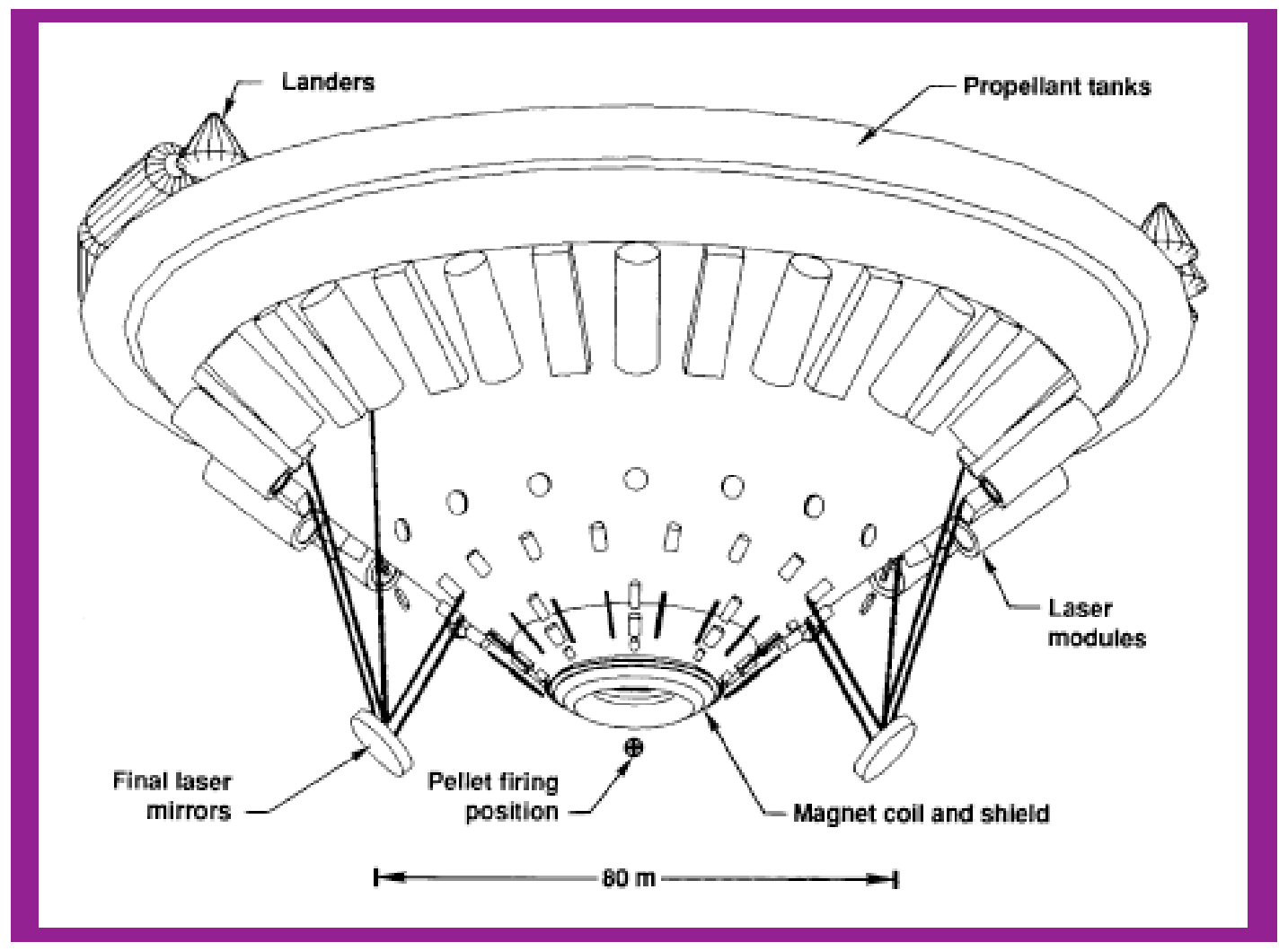


This document was prepared as an account of work sponsored by an agency of the United States Government. Neither the United States Government nor the University of California nor any of their employees, makes any warranty, express or implied, or assumes any legal liability or responsibility for the accuracy, completeness, or usefulness of any information, apparatus, product, or process disclosed, or represents that its use would not infringe privately owned rights. Reference herein to any specific commercial product, process, or service by trade name, trademark, manufacturer, or otherwise, does not necessarily constitute or imply its endorsement, recommendation, or favoring by the United States Government or the University of California. The views and opinions of authors expressed herein do not necessarily state or reflect those of the United States Government or the University of California, and shall not be used for advertising or product endorsement purposes.

This work was performed under the auspices of the U.S. Department of Energy by University of California, Lawrence Livermore National Laboratory under Contract W-7405-Eng-48. 


\section{VIST杰}

A $V$ ehicle for Interplanetary $S_{\text {pace }} \Gamma_{\text {ransport }}$

Application Powered By Inertinl Confinement Fusion

Charles D. Orth, PhD

Systems Analysis Final Report

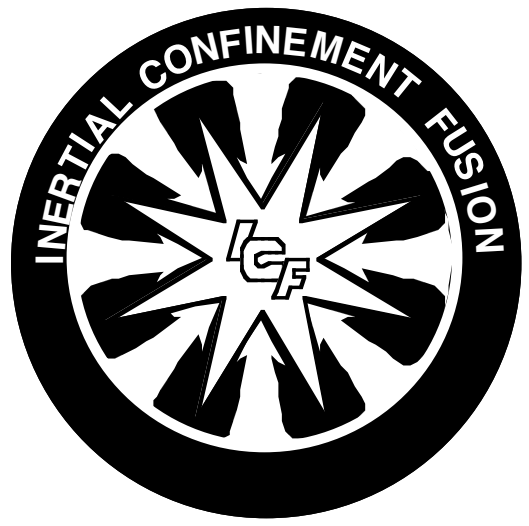

May 16, 2003

LAWRENCE LIVERMORE NATIONAL LABORATORY

University of California $・$ Livermore, California $\bullet$ 94551-0808 


\title{
NOTE:
}

\section{Original Manuscript Number} UCRL-LR-110500

\author{
LLNL IM System Number \\ UCRL-TR-110500 \\ Request for copies from LLNL \\ may use either number.
}




\section{TABLE OF CONTENTS}

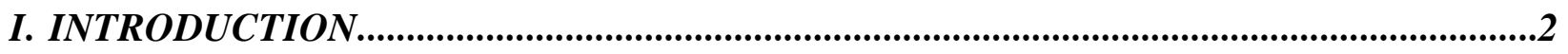

I.1 Transport Concepts for Mars Missions....................................................................................2

I.2 The Need to Consider Fusion for Space Propulsion ........................................................................

I.3 The Development of Fusion-Powered Spacecraft Concepts..........................................................5

I.4 The VISTA Concept ......................................................................................................................6

I.5 Intent and Overview of This Report....................................................................................

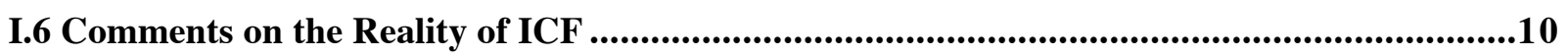

II. THE NATURE OF INERTIAL CONFINEMENT FUSION (ICF) .................................12

II.1 What is fusion?..........................................................................................................................12

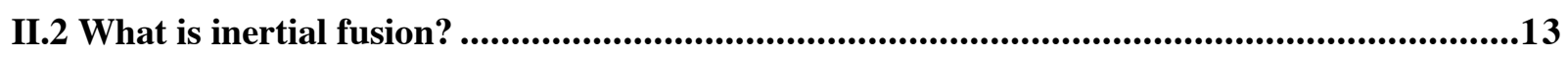

II.3 The Advantages of ICF...................................................................................................14

II.4 Why don't we have a terrestrial ICF power plant now? .........................................................15

II.5 Why Consider Fusion for Space Propulsion Systems?.............................................................16

II.6 Advanced ICF target concepts............................................................................................17

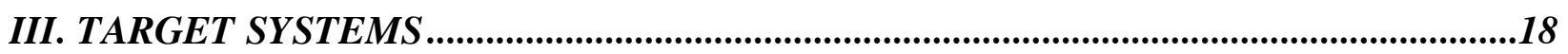

III.1 Capsule Design .........................................................................................................................18

III.2 Expellant..............................................................................................................19

III.3 Target Fabrication and Assembly .....................................................................................20

III.4 Target Acceleration, Injection, Tracking, and Beam Pointing.............................................20

III.5 Target Performance (Gain) .....................................................................................................22

III.6 Target Emissions............................................................................................................24

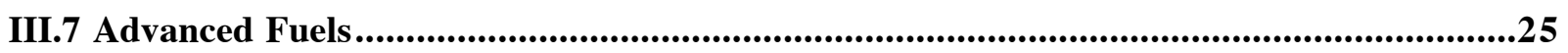

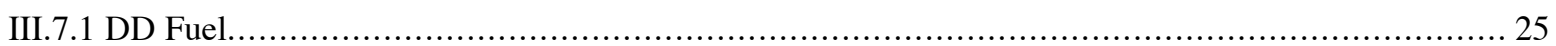

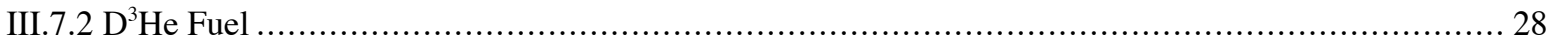

III.7.3 General Comments about Advanced Fuels and Antimatter ....................................... 28

III.8 Target and Propellant Storage ...........................................................................................29

III.9 Onboard Production of Tritium ............................................................................................30

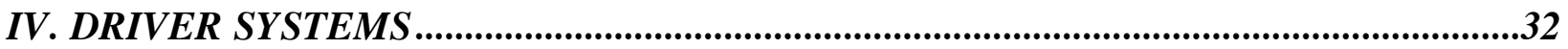

IV.1 A Survey of Terrestrial Driver Developments......................................................................32

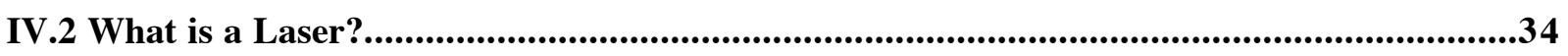

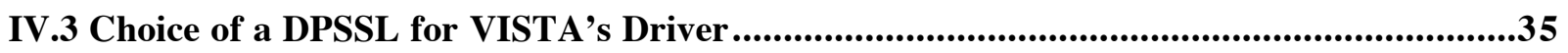

IV.4 A Conceptual Driver Alternative ......................................................................................38

IV.5 Beam Line Optics and Focusing .............................................................................................40

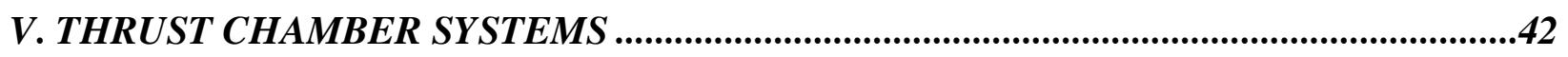

V.1 System Configuration and Basic Engine Operation..............................................................43

V.2 Superconducting Magnet ...................................................................................................44

V.3 Coil Shield.......................................................................................................................................4 
V.4 X-Ray Ablation Protection for First Wall \& Final Mirrors .............................................48

V.5 Target Debris Plasma Physics.................................................................................................49

V.6 Plume Formation and Plasma Collection Efficiency ...........................................................51

V.7 Jet Efficiency ...........................................................................................................51

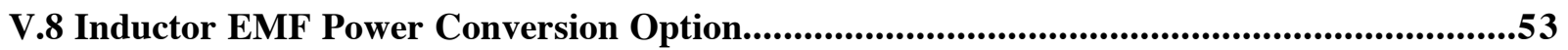

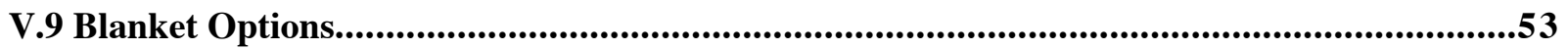

V.10 Shock Isolation......................................................................................................54

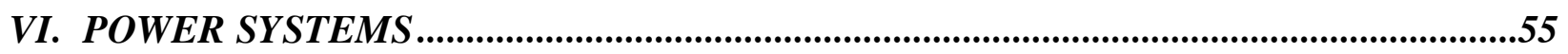

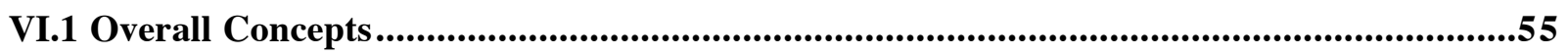

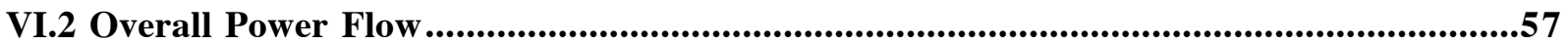

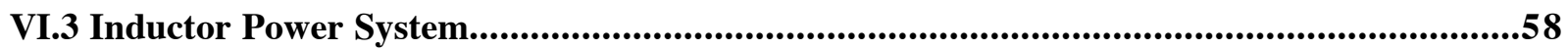

VI.3.1 Inductor Power Conversion Subsystem ............................................................ 59

VI.3.2 Inductor Power Conditioning Subsystem......................................................... 59

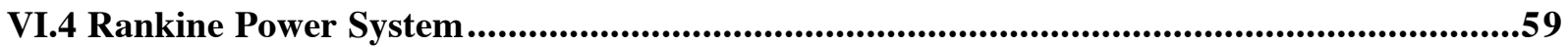

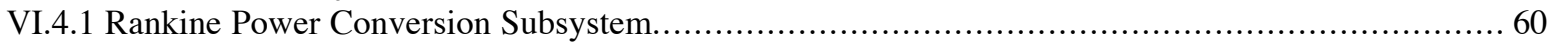

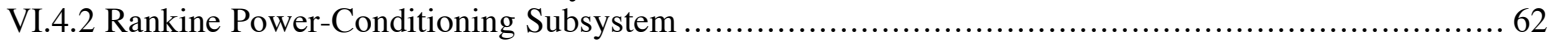

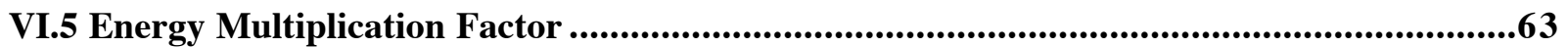

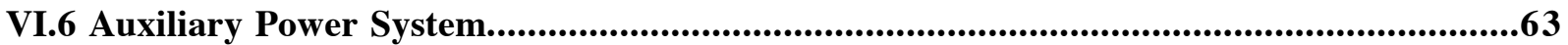

VI.7 Engine Startup Power System................................................................................64

VI.8 Uncertainties in Power Generation and Their Effects..........................................................64

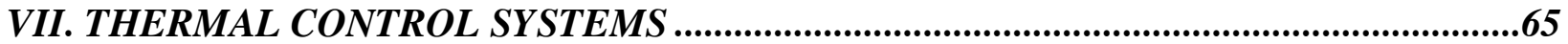

VII.1 Concepts and Effects of Technology Development..........................................................65

VII.2 Radiator Design With Micrometeoroid Shields......................................................66

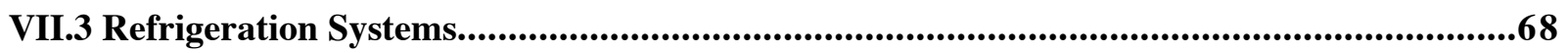

VII.4 Thermal Systems for the Magnet Coil Shield...................................................................68

VII.5 Thermal Systems for the Final Laser Mirrors....................................................................68

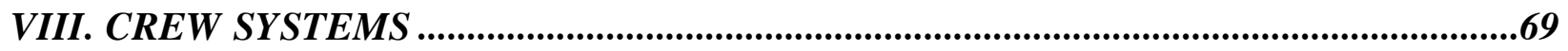

VIII.1 Types of Crew Systems.........................................................................................69

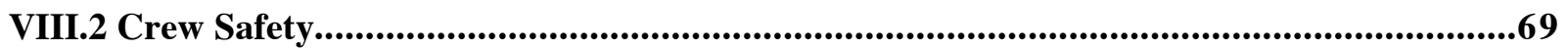

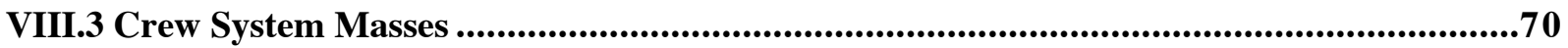

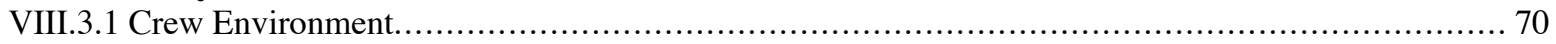

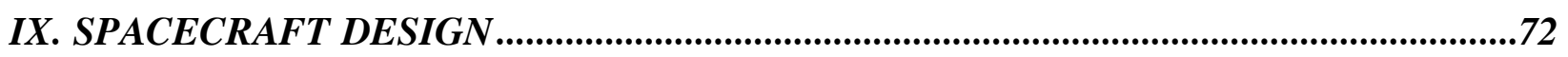

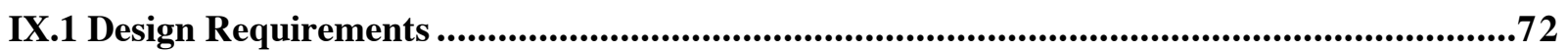

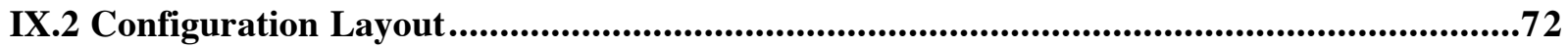

IX.3 Final Turning Mirror Structure ............................................................................................74

IX.4 Integration of Conical Shell and Radiator Structure ......................................................74

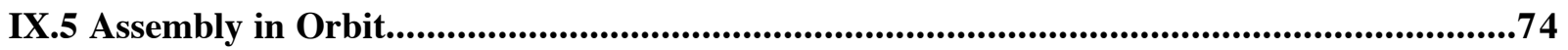

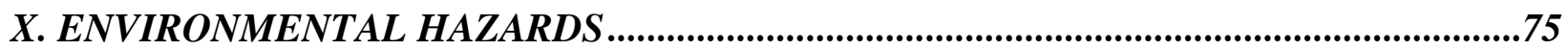

X.1 Tritium Hazards ...................................................................................................................75

X.1.1 Tritium Hazards for Fuel Transport Up Through the Atmosphere ...................................... 75 
X.1.2 Tritium Hazards for Onboard Crew Members................................................................. 79

X.2 Neutron Activation of Spacecraft Components............................................................79

X.3 Neutron Hazards to Other Spacecraft ......................................................................80

X.4 Exhaust and Particulate Hazards to Other Spacecraft ....................................................80

XI. TRAJECTORY ANALYSIS FOR MARS MISSIONS ...............................................81

XI.1 Simple Approximate Analysis.........................................................................................81

XI.2 Differences From Our Previous Publications .......................................................................85

XI.3 Loss in Performance due to Mass Contingencies ..........................................................85

XI.4 Loss in Performance due to ALL System Uncertainties................................................87

XII. TRAJECTORY ANALYSIS FOR MISSIONS BEYOND MARS...............................89

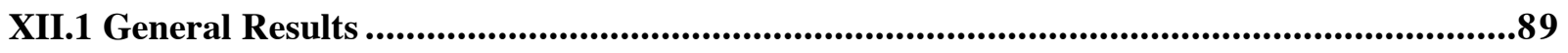

XII.2 Loss in Performance due to ALL System Uncertainties..............................................89

XIII. MISSIONS BEYOND THE SOLAR SYSTEM.................................................91

XIV. DEVELOPMENT STRATEGY and TECHNOLOGY READINESS ...........................93

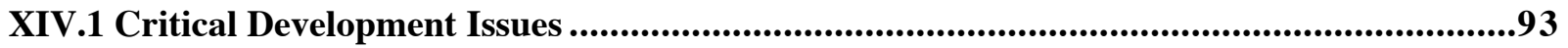

XIV.2 Current Status ....................................................................................................................95

XIV.3 Realistic Development Schedule and Costs.....................................................................96

XIV.4 Recommendation for Studies of Ways to Reduce Tritium Requirements ........................98

XIV.5 Preliminary Technology Readiness Assessment.............................................................99

XIV.6 A Fast-Track Approach to Fusion Propulsion ......................................................100

XIV.6.1 Synergism Possible From a NASA-DOE Dual Approach............................................100

XIV.6.2 Differences Between Terrestrial and Space Applications................................................ 101

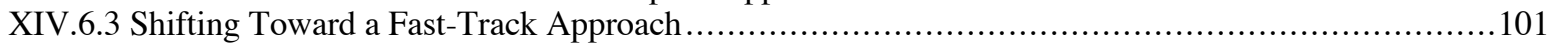

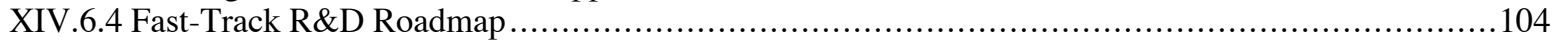

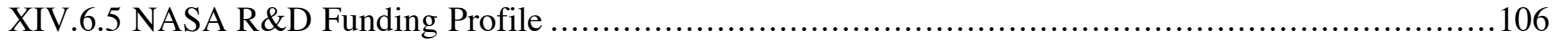

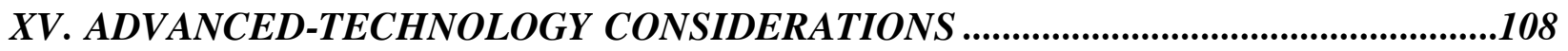

XV.1 Near-Future Advancements........................................................................................108

XV.2 Far-Future Advancements ............................................................................................109

XVI. COMPARISON WITH OTHER TECHNOLOGIES..........................................111

XVI.1 Magnetic Confinement Fusion (MCF) ............................................................................111

XVI.2 Nuclear Electric Propulsion (NEP) Concepts ........................................................112

XVI.3 Antimatter-Initiated Fission/Fusion......................................................................112

XVI.4 Orion-Type Pusher-Plate Concepts Using Nuclear Explosions....................................113

XVI.5 General Considerations ...................................................................................................113

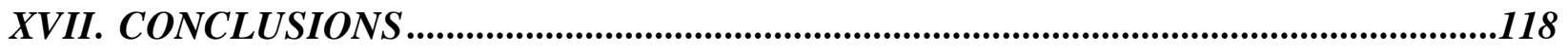

APPENDIX A：HIGH-PERFORMANCE SPACECRAFT SCALING ............................119

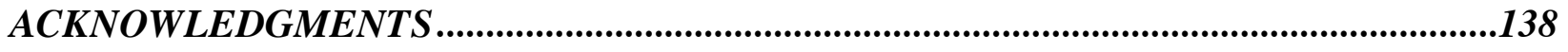

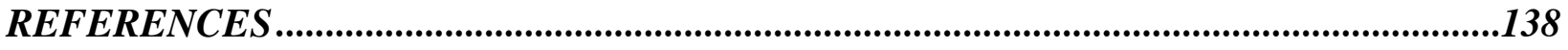




\section{LIST OF FIGURES}

I-1 The VISTA Spacecraft Configuration $\ldots \ldots \ldots \ldots \ldots \ldots \ldots \ldots$

III-1 A Generic Type of Target for Indirect Drive .............. 18

V-1 Magnetic Thrust Chamber Operation .................. 42

V-2 Superconducting Magnet/Shield Conceptual Design .......... 45

VI-1 Overall Power Flow with Inductor Power System. . . . . . . . . . 57

VI-2 Overall Power Flow with Ranking Power System. .......... 58

VI-3 Rankine-Cycle Power Flow Diagram .................... 60

XI-1 Comparison of Approximate/Exact Alpha Curves for Mars ..... 82

XI-2 Performance Variation with Driver Efficiency, Radiator Tech.... 84

XI-3 Performance Variation with Target G, Driver T, Payload M. .... . 84

XIV-1 Terrestrial IFE Development Strategy . . . . . . . . . . . . . . . . 97

XIV-2 DPSSL Integrated Research Experiment Configuration ......... 98

XIV-3 Roadmap for Development of Nuclear Fusion Propulsion ...... 105

XIV-4 Estimated NASA Fast-Track Direct-Cost Funding Profile . . . . . . . 107 


\section{LIST OF TABLES}

I-1 General Assumptions for Project VISTA $\ldots \ldots \ldots \ldots \ldots \ldots \ldots$

II-1 Attributes of Various Power Sources . . . . . . . . . . . . . . 17

III-1 Target Gains for Different Fuel Masses . . . . . . . . . . . . . . . . . 24

IV-1 Desired Features of VISTA's Target Driver............... 32

IV-2 Excimer Laser Processes . . . . . . . . . . . . . . . . . . . . . . . . . . 39

V-1 Assumed Magnet Coil Matrix Composition ................ 46

V-2 Magnet Coil Parameters ........................... 46

V-3 Magnet and Shield Composition .................... 47

XI-1 Parameters Assumed for Advanced-Technology Case ......... 82

XI-2 Distribution of Mass for Advanced Mission to Mars ........... 83

XI-3 Effect of mass contingencies on Mars Roundtrip Performance... $\quad 85$

XI-4 VISTA Mass Properties Table With Contingencies ........... 86

XI-5 Monte Carlo Variations in Trip Times to Mars ............. 88

XII-1 Mission Durations to the Planets for Different Target Gains .... $\quad 89$

XII-2 Monte Carlo Variations in Trip Times to Jupiter and Pluto ..... $\quad 90$

XIII-1 DT Missions Beyond Pluto ...................... 91

XIII-2 Time and Kinetic Energy Required to Get to $10^{4}$ AU. .......... 91

XIV-1 Development Issues for VISTA $\ldots \ldots \ldots \ldots \ldots \ldots \ldots \ldots \ldots . . \ldots 3$

XIV-2 Technology Readiness Level Estimates .................. 100

XIV-3 One Possible DOE Roadmap for IFE ................. 102

XIV-4 ICF Fusion-Propulsion Milestones and Key Decision Points ..... 103

XIV-5 NASA Fast-Track Fusion Propulsion Development Funding .... 106

XV-1 Mars Missions With Different Target Fuels ............... 108

XV-2 Mars Missions With Antimatter Fuel .................. 109 


\title{
PREFACE
}

This document represents the final report for the original VISTA systems study conducted in 1986 and 1987 by the following individuals, who formed the systems analysis team:

\author{
Charles D. Orth \\ Lawrence Livermore National Laboratory, Livermore, CA \\ Gail Klein and Joe Sercel \\ Jet Propulsion Laboratory, Pasadena, CA \\ Nathan Hoffman and Kathy Murray \\ (Formerly of) ETEC/Rocketdyne/Rockwell, Canoga Park, CA \\ Franklin Chang Diaz \\ NASA, Astronaut Office, Johnson Space Center, Houston, TX
}

Many other people in addition to those listed above contributed to this original study, including Bill Hogan and Max Tabak at the Lawrence Livermore National Laboratory (LLNL) and Hoppy Price, Stan Krauthamer, Carl Sauer, Andrey Sergeyevsky, Katie Barhydt, Alok Chatterjee, and Jayant Sharma at the Jet Propulsion Laboratory (JPL).

The original study was conducted to determine whether inertial confinement fusion (ICF) could be adapted for piloted space transport to Mars with a sufficient increase in speed over conventional rocket technology that the physiological deteriorations from exposures to zero gravity and cosmic rays could be reduced to a manageable level. The study was very successful, and produced the VISTA spacecraft concept, which is still the leading ICF-powered spacecraft concept in the world.

Since the late 1980's, the lead author has made extensive additions and improvements to the original study by (1) making the system mass estimates more realistic, (2) detailing the design of certain systems such as the superconducting magnet in the magnetic thrust chamber, (3) writing his own simple analytic mission-trajectory computer code to do approximate but comprehensive systems analysis combining both spacecraft system masses and mission trajectories, (4) extending the results to destinations other than Mars, and (5) writing and assembling this report. These extensions proved to be very important. For example, the minimum mission duration for a roundtrip to Mars has increased from 90 days to a more realistic 145 days. This document therefore includes a description of these enhancements and the extended mission analyses in addition to the methodology used in the original systems study. 


\title{
VISTA--A Vehicle for Interplanetary Space Transport Application Powered by Inertial Confinement Fusion
}

\begin{abstract}
Inertial Confinement Fusion (ICF) is an ideal technology to power self-contained single-stage piloted (manned) spacecraft within the solar system because of its inherently high power/mass ratios and high specific impulses (i.e., high exhaust velocities). These technological advantages are retained when ICF is utilized with a magnetic thrust chamber, which avoids the plasma thermalization and resultant degradation of specific impulse that are unavoidable with the use of mechanical thrust chambers. We started with Rod Hyde's 1983 description of an ICF-powered engine concept using a magnetic thrust chamber, ${ }_{1}^{1}$ and conducted a more detailed systems study to develop a viable, realistic, and defensible spacecraft concept based on ICF technology projected to be available in the first half of the $21^{\text {st }}$ century. The results include an entirely new conical spacecraft conceptual design utilizing near-existing radiator technology. We describe the various vehicle systems for this new concept, estimate the missions performance capabilities for general missions to the planets within the solar system, and describe in detail the performance for the baseline mission of a piloted roundtrip to Mars with a 100-ton payload. For this mission, we show that roundtrips totaling $\geq 145$ days are possible with advanced DT fusion technology and a total (wet) spacecraft mass of about 6000 metric tons. Such short-duration missions are advantageous to minimize the known cosmic-radiation hazards to astronauts, and are even more important to minimize the physiological deteriorations arising from zero gravity. These ICF-powered missions are considerably faster than those available using chemical or nuclear-electric-propulsion technologies with minimum-mass vehicle configurations. VISTA also offers onboard artificial gravity and propellant-based shielding from cosmic rays, thus reducing the known hazards and physiological deteriorations to insignificant levels. We emphasize, however, that the degree to which an ICF-powered vehicle can outperform a vehicle using any other realistic technology depends on the degree to which terrestrialbased ICF research can develop the necessary energy gain from ICF targets. With aggressive progress in such terrestrial research, VISTA will be able to make roundtrip missions to Pluto in $\sim 7$ years, and missions to points just beyond the solar system within a human lifetime.
\end{abstract}




\section{INTRODUCTION}

Man has always had a fascination for space travel and exploration of the universe. Great progress has already been made in exploring the Earth and the moon with piloted (manned) and unpiloted (unmanned) space missions, and in exploring other nearby bodies in the solar system using unpiloted probes. The focus for piloted transport in the next few decades will undoubtedly be missions to Mars-provided the desire and will to go there can kindle the development of the appropriate technology to get astronauts there and back in a safe manner.

Specifically, in 1990, President Bush approved a National Space Policy Directive reaffirming the commitment of the United States to space exploration, and outlining the steps to achieve the 1988-1992 Space Exploration Initiative (SEI) that included both piloted and unpiloted missions to the Moon and to Mars. His desire was to achieve piloted exploration of Mars by AD 2019. NASA therefore considered strategies for piloted interplanetary missions, specifically, a series of roundtrips to the surface of Mars, leading to the eventual establishment of a permanent Mars base. ${ }^{2}$ More recently, NASA's Jet Propulsion Laboratory (JPL) sent an orbiter (Mars Global Surveyor) and a lander (Mars Pathfinder) to Mars in 1996, and plans to fly two missions to Mars about every 26 months through 2005 as part of its Mars Exploration Program. Thereafter, the mission emphasis will probably shift to more distant planets and missions beyond the solar system.

Although much experience will presumably be required during Shuttle, lunar, and Space-Station missions prior to any piloted mission to another planet, it seems likely that a piloted mission to Mars will be the ultimate focus of the U.S. space program for the early 21st century. ${ }^{3}$ In this report, we focus on such interplanetary transport, and consider a piloted mission to Mars as the most relevant interplanetary mission.

\section{I.1 Transport Concepts for Mars Missions}

Most discussions of vehicle concepts for interplanetary transport have assumed the use of chemical fuels, with some mention of nuclear-fission-powered engines. Such a focus reflects near-term technology, which permits roundtrip missions to Mars with flight durations of typically one to three years using minimum-mass configurations. Such flight durations might seem satisfactory, except that the U.S. space flights already completed have demonstrated that space is a very hazardous radiation environment for such lengthy missions, ${ }^{4}$ and USSR Souyez flights have demonstrated that man's skeletal physiology suffers significantly for durations exceeding about 100 days in zero gravity. Longer flight durations result not only in astronauts being unable to stand up or walk without assistance, but also in a range of associated blood and physiological symptoms. ${ }^{5}$ In addition, for flights exceeding roughly one year, the galactic cosmic radiation produces significant physiological deterioration, resulting in predicted leukemias and associated ailments. ${ }^{6}$ Typically, any given human cell will be struck once every three days by a cosmic-ray proton, once a month by a helium ion, once in 9 years by a heavy ion with charge between 10 (neon) and 26 (iron), and 6\% of all cells will be hit two or more times by such heavy nuclei in a threeyear mission. ${ }^{7}$ Thus, potential physiological problems are becoming the most pressing concern of astronauts. Astronauts are therefore emphasizing the need for propulsion systems that will shorten mission durations. 
There are hence two development strategies for piloted missions to Mars carrying a 100-ton payload: 1) consider more advanced technologies so that total roundtrip missions of $<100$ days are possible, thereby directly avoiding both the significant radiation-hazard effects and the physiological deterioration; or 2) use a vehicle concept that includes large masses for radiation shielding and that also includes a capability for artificial gravity, thereby reducing the hazards for trips exceeding 100 days to insignificant levels. Technical issues involved in assessing the merits of these two strategies include whether the more advanced technologies are even feasible, and whether they require a greater increase in engine mass than would be incorporated in the radiation-shielding mass needed for more lengthy missions using conventional technologies. Economics, politics, and the innate desire for fast trips must also be considered. For this report, we shall assume that strategy \#1 (transport in $<100$ days) is beyond the reach of any technology for the foreseeable future (i.e., we believe that no concept will survive a realistic systems study and produce this kind of performance, because fission, fusion, and antimatter systems cannot achieve such performance with $21^{\text {st }}$-century technology). The challenge is thus how to use strategy \#2 and yet have trip times as close as possible to 100 days.

Given sufficient technological development, a relatively fast mission to Mars can be implemented with many different overall transport concepts, considering vehicle staging, multiple vehicles, specialized trajectories, etc. The concept we consider in this report is the usual self-contained single-stage vehicle that must supply its own power for propulsion. With conventional (non-nuclear) engines, this concept excludes fast missions to Mars because the added mass of the onboard engine cannot be offset with a suitably large engine output power to afford a large enough power/mass ratio. With nuclear fusion, however, we have been able to develop a viable systems concept that does have suitable power/mass ratio. Fusion is about 40 billion times more efficient in converting mass into energy than chemical reactions, and 4 times more efficient than nuclear fission. In addition, the extra engine equipment required to use fusion is not excessive. As a result, fusion technology - if successfully developed - can reduce the flight duration for a roundtrip to Mars to about 145 days with a self-contained single-stage spacecraft concept. Nuclear fusion can even permit roundtrip missions to Pluto in less than 8 years. Consequently, use of fusion as a power source can allow the flexibility in launch times and flight paths available with a self-contained vehicle, and permit safe fast transport within the interplanetary system.

Another way to accomplish fast interplanetary transport is to assume that the power source for the vehicle is fixed somewhere in the Earth-Moon system, such as on the lunar surface, ${ }^{8}$ and that power beamed to the spacecraft from this location is used to accelerate onboard propellant. This concept permits significantly higher spacecraft power-to-mass ratios because there is less onboard mass for engine and waste-heat-rejection equipment. Shorter trip times are therefore possible, and launch times are also flexible, but there are constraints on how far away the vehicle can go, and restricted capability to explore regions behind planets or other occulting objects.

A third concept for fast transport involves cycling spacecraft and transfer vehicles. In this concept, the cycling spacecraft is in a permanent eccentric orbit around the sun, and a transfer vehicle must take a payload from a lunar-libration-orbit spaceport (at the point of equal Earth and Moon gravity) up to meet the cycling spacecraft just as the cycling spacecraft passes by the Earth on its way out to Mars, as it does once every 4 years. ${ }^{9}$ The payload would stay with the cycling ship for the 5 to 7-month trip out to Mars, and then 
decouple for a transfer to the appropriate Mars orbit. The return trip to Earth would require waiting about one year on the planet to catch the cycling spacecraft on its way back toward the sun. This concept may save resources for many missions, but it restricts launch opportunities and choices for destinations (even with multiple cycling spacecraft), involves another crew which must be in space for extended periods, prolongs the stay on Mars, and limits how fast the trip can be made. A variation of this concept is to use rotating tethered systems circling Earth and Mars, ${ }_{10}^{10}$ but such systems assume pickup and dropoff of payloads at about $2 \mathrm{~km} / \mathrm{s}$ in eccentric sub-orbital planetary orbits using tethered masses roughly 15 times more massive than the payload.

Although these and other concepts permit fast interplanetary transport, we chose to consider only the first concept, that is, a self-contained single-stage spacecraft. In particular, the objective for the study reported here was to specify a viable vehicle systems concept for piloted missions within the solar system that would incorporate technology available in the first half of the $21^{\text {st }}$ century and enable transport of 100-ton payloads to Mars and back in a time as close as possible to 100 days. We included the 100-day criterion to minimize the physiological deteriorations to astronauts resulting from zero gravity, as well as the induced-cancer hazards resulting from in-flight cosmic-radiation exposures, even though we planned to develop a concept that could provide artificial gravity and cosmic-ray shielding for safe missions of longer durations. An additional purpose for including the near-100-day requirement was to demonstrate the advantages of nuclear fusion, because such fast relatively near-term piloted missions cannot be accomplished using minimummass configurations and either chemical or nuclear-fission technologies. As it turned out, our design not only demonstrates the need for the development of fusion propulsion, but also allows safe transport to any point within the solar system even though the roundtrip flight durations exceed the 100-day requirement.

\section{I.2 The Need to Consider Fusion for Space Propulsion}

With our current understanding, propulsion systems based on nuclear fusion are expected to provide the fastest transport to Mars in the $21^{\text {st }}$ century. Systems based on nuclear fission (e.g., nuclear-electric propulsion), although closer to reality than fusion systems, can only provide roundtrips to Mars of two-thirds of a year or more-certainly nothing close to 145 days. Thus, if man continues to emphasize speed (for whatever reasons), the development of fusion propulsion systems appears to be a necessity. We return to a discussion of the advantages of fusion in Sec. II.1, where we consider the subject in more detail. A basic understanding of the advantages of fusion is helpful at this point, however, so we now explain what fusion is and why it is so desirable.

Nuclear fusion is the process in which a small amount of mass is converted into energy through $\mathrm{E}=\mathrm{mc}^{2}$ when light elements (e.g., a deuterium isotope $\mathrm{D}$ and a tritium isotope $\mathrm{T}$ of hydrogen) are combined to produce heavier elements (e.g., helium He plus a neutron): D + $\mathrm{T}-->\mathrm{He}+\mathrm{n}+$ energy. This reaction releases $17.6 \mathrm{MeV}$, or $2.8 \times 10^{-12}$ joule per interaction, which amounts to about one hundred billion joules per gram of fuel (at $1 / 3$ burnup). Said differently, $0.45 \mathrm{~kg}$ (one pound) of fusion fuel releases as much energy as about 370,000 gallons of petroleum. Thus, although it might take 2,500,000 tons of coal or 160,000,000 gallons of oil or $25,000 \mathrm{~kg}$ of UO fuel to operate a 1000-MW terrestrial power plant for one year, it would take only about $200 \mathrm{~kg}$ of DT fusion fuel. Such huge specific energy outputs afford very high power/mass ratios (ten to several hundred watts per gram) and very high 
specific impulses (tens of thousands of seconds). High power/mass ratios permit high accelerations up to a speed at which a vehicle can coast, thereby conserving fuel for faster trips. Specific impulse is the ratio of the effective exhaust velocity $(\mathrm{m} / \mathrm{s})$ and the acceleration due to gravity $\left(9.8 \mathrm{~m} / \mathrm{s}^{2}\right)$, and hence has units of "seconds." High specific impulses enable high maximum vehicle velocities, and thus shorter trip times. Fusion thus has great advantages for interplanetary missions.

Before fusion can occur, fusion fuel must be heated (either by compression or external heating) to a temperature of about $10 \mathrm{keV}$ (i.e., about 100 million degrees K) and confined in its heated state long enough to exceed a minimum product of density and confinement time. If the fusion fuel is compressed to high density and confined for nanoseconds by its own inward-moving inertia, the fusion process is called Inertial Confinement Fusion (ICF); if the fusion fuel is not compressed but heated by external means and confined for many seconds by a magnetic field, the fusion process is called Magnetic Confinement Fusion (MCF). We will only consider ICF here, because it has already been shown to work with sufficient compressional energy input. In ICF, a "capsule" containing the fusion fuel is heated either indirectly or directly by lasers or ion beams from a "driver," which supplies the energy needed to compress the fuel (Section II.2 describes ICF in more detail).

\section{I.3 The Development of Fusion-Powered Spacecraft Concepts}

The application of ICF to rocket propulsion began in the early 1970's. Balcomb, et al. ${ }^{11}$ at the Los Alamos National Laboratory (LANL) proposed a laser-fusion concept that retained the idea of acquiring acceleration through particles striking a pusher plate. The pusher-plate idea originated in Project Orion, ${ }^{12}$ in which nuclear explosions were detonated behind a massive plate attached to the spacecraft through a pneumatic spring system. Then Hyde, Wood, and Nuckolls ${ }^{13}$ at the Lawrence Livermore National Laboratory (LLNL) proposed the use of laser fusion with a magnet to redirect the charged-particle debris from the fusion microexplosions, so that the debris would exit the rear of a thrust chamber and provide thrust. In their concept, the debris never touched any vehicle structure, and was hence never forced to thermalize with other materials. Avoidance of thermalization permitted specific impulses in the range from $10^{5}$ to $10^{6}$ seconds, which were much larger than the $10^{4}$ obtainable with the LANL concept.

At about this same time, Winterberg ${ }^{14}$ proposed the use of relativistic electron beams to initiate the fusion microexplosions, with a concave mirror reflector open on one side to redirect the debris. Winterberg's work motivated a large systems study by the British Interplanetary Society, called Project Daedalus. ${ }^{15}$ The Daedalus 2-stage propulsion system employed relativistic electron beams plus a magnetic reaction chamber. The objective of Project Daedalus was to design a vehicle to be able to go to Barnard's Star 6 light years away with a one-way trip time (without deceleration at the destination) of 50 years at about $12 \%$ of the speed of light.

Rod Hyde ${ }^{16}$, expanding on the previous LLNL work, developed a complete concept of a laser-fusion-powered pencil-shaped vehicle for interplanetary transport that uses DD fuel and assumes a capsule energy gain of 1000 at a driver energy of 2 MJ. His thrust chamber incorporated a 16-Tesla superconducting magnet to redirect the capsule debris, and operated at $100 \mathrm{~Hz}$ with an assumed jet efficiency of $42 \%$. His so-called VIP missions, with essentially no payload mass, allowed roundtrip times to Mars in less than 3 weeks, ignoring 
the significant effects of solar and planetary gravities; his so-called cargo missions to Mars would take only 45 days. These mission times would be larger if gravitation were included.

\section{I.4 The VISTA Concept}

In 1986, we began with Hyde's concept and conducted a detailed systems study subject to the assumptions listed in Table I-1 below. The study team included scientists with expertise in ICF capsule design, spacecraft trajectory analysis, thermal systems, power systems, mechanical systems, and actual Shuttle flight experience. The study was intended to identify the long-term mission benefits for NASA's advanced missions planning, and to identify the required technological development challenges as an aid to future technology monitoring, concept re-evaluations, and systems viability assessments. The total mass of the spacecraft had to be consistent with the weight capability of NASA's Heavy-Lift Launch Vehicle for assembly in orbit (then 200 tons per lift). Of particular interest was a pilotedMars mission, so we chose this as the baseline mission. In subsequent sections of this report, we give detailed results for this baseline mission, plus estimates of performance for missions to the outer planets and beyond. Our concept is called VISTA (Vehicle for Interplanetary Space Transport Applications). We assume the use of technology available by $\mathrm{AD} 2050$ or before, assessed as a reasonable and viable extrapolation of existing technology; nevertheless, we believe that this technology base could be developed by AD 2020 if there were sufficient national commitment. We label such technology as "early $21^{\text {st }}$ century technology."

\section{Table I-1. General Assumptions for Project VISTA.}

1. Use of a self-contained single-stage vehicle which carries its own power-generation equipment (as opposed to power transmission from a lunar base, etc.) and which carries the payload through the entire trip without the use of other orbit-transfer vehicles.

2. Use of flight trajectories in interplanetary space that include the effects of gravity.

3. Use of early $21^{\text {st }}$-century technology for all systems. In particular, we assumed the availability of a laser driver with a delivered output of $0.50 \mathrm{~W} / \mathrm{g}$, capsule gains in the range from 200 to 1500, and heat-pipe radiators with 0.07 to $0.15 \mathrm{~kg} / \mathrm{kWt}$ at $1000 \mathrm{~K}$.

4. Minimum costs (if possible) for R\&D, assembly, and operation, but we did not let costs become an overriding consideration. For example, we did not worry about the purchase or generation cost of 2.2 metric tons of tritium, but we assumed that $R \& D$ costs would be minimized by the compatible terrestrial R\&D development.

5. Use of a micrometeoroid model that is $10 \%$ worse than that given by the 1970 NASA model ${ }^{17}$.

6. No limit on total mass. However, we assumed that greater acceptance of a vehicle concept would occur if fewer launches by NASA's Heavy Lift Launch Vehicle (or equivalent) were required for assembly in orbit (at about 200 tons per launch).

7. Use of tritium is acceptable. That is, we assumed the incorporation of safety precautions and procedures that can reduce the risks of human exposure to tritium to acceptable levels during boost into orbit, assembly, and vehicle operation.

8. Use of DT fuel is acceptable. That is, we assumed the incorporation of safety precautions, procedures, and shields for personnel in nearby spacecraft to avoid physiological damage from solar-flare particles and cosmic-ray-induced neutron showers experienced in their spacecraft, thereby permitting the use of DT in VISTA even though VISTA emits copious neutrons as it accelerates away 
from another spacecraft. We merely assumed that the personnel in the nearby spacecraft can hide behind their own shields for a period of 6 to 12 hours until VISTA gets far enough away that the neutron exposure is no longer a hazard.

9. Artificial gravity is required for all flights exceeding roughly 100 days (to avoid skeletal and other physiological deteriorations in crew members).

10. Crew shielding against cosmic-ray-initiated neutron cascade showers is required for all flights exceeding $1 / 2$ to 1 year (to avoid significant rates of induced leukemias and other cancers).

Our results include an entirely new spacecraft concept (Fig. I-1), which can be described as follows. A 13-m-radius superconducting magnet generates a magnetic field that has a peak field of about 12 Tesla and defines the boundaries of a thrust chamber. Preassembled marble-size fuel capsules are filled enroute with cryogenic deuterium (D) and tritium (T), which are isotopes of hydrogen. The fuel capsules surrounded by about $50 \mathrm{~g}$ of added hydrogen mass (called the expellant) are accelerated, injected, and positioned at the center of the thrust chamber at a repetition rate of 0 to $30 \mathrm{~Hz}$, depending on the desired throttling (i.e., acceleration). Beams from a laser delivering about $5 \mathrm{MJ}$ with an efficiency of at least $6 \%$ are focused on the capsule, causing it to implode and release up to 7,500 MJ in neutron, $\mathrm{x}$-ray, and plasma-debris energy. The conical spacecraft design permits nearly all of the neutron and x-ray energy to escape to space because the spacecraft structure lies in the shadow of the thermal shield for the superconducting magnet coil. Only a small part $(4 \%)$ of the emitted capsule energy strikes the shield protecting the superconducting coil, and must be radiated as waste heat or used in a Rankine power cycle. The crew quarters reside inside the propellant tanks at the outer lip of the conical structure, and are thereby shielded from cosmic radiations. Crew access is thereby slowly restricted as the mission proceeds because the consumption of propellant removes the shielding in those regions of the spacecraft lip that have been depleted of propellant. The entire structure can be rotated about its axis to provide artificial gravity (e.g., $3 \mathrm{rpm}$ provides 1 gee at $\mathrm{R}=100 \mathrm{~m}$ ).

In the VISTA concept, the capsule debris, being conductive, expands spherically until the plasma pressure drops below the magnetic pressure. The debris is then deflected by the magnet, which stores an energy that is five times the debris kinetic energy. The debris, which is recombining and decreasing in conductivity, exits the thrust chamber in a limited solid angle, producing thrust and propelling the spacecraft with its blunt end forward. By simply changing the repetition rate, the ICF engine has variable thrust capability. By changing the amount of expellant mass surrounding each capsule (or by changing the capsule design), the ICF engine has variable specific-impulse capability. We here assume that the capsule gain (the ratio of the energy produced by the fusion to the driver energy supplied to the capsule) can be as large as 1500 for very advanced DT technology [see Sec. III.5, Eq. (4)]. Such gains allow the ICF engine to have a power $/$ mass ratio of about $20 \mathrm{~W} / \mathrm{g}$ at a repetition rate of $30 \mathrm{~Hz}$. The avoidance of debris thermalization with surrounding structures allows effective specific impulses (i.e., specific impulses multiplied by the squareroot of the jet efficiency) near 20,000 seconds. 
Figure I-1: The VISTA Spacecraft Configuration. Two final laser focusing mirrors are shown, but optional configurations incorporate one or more mirrors.

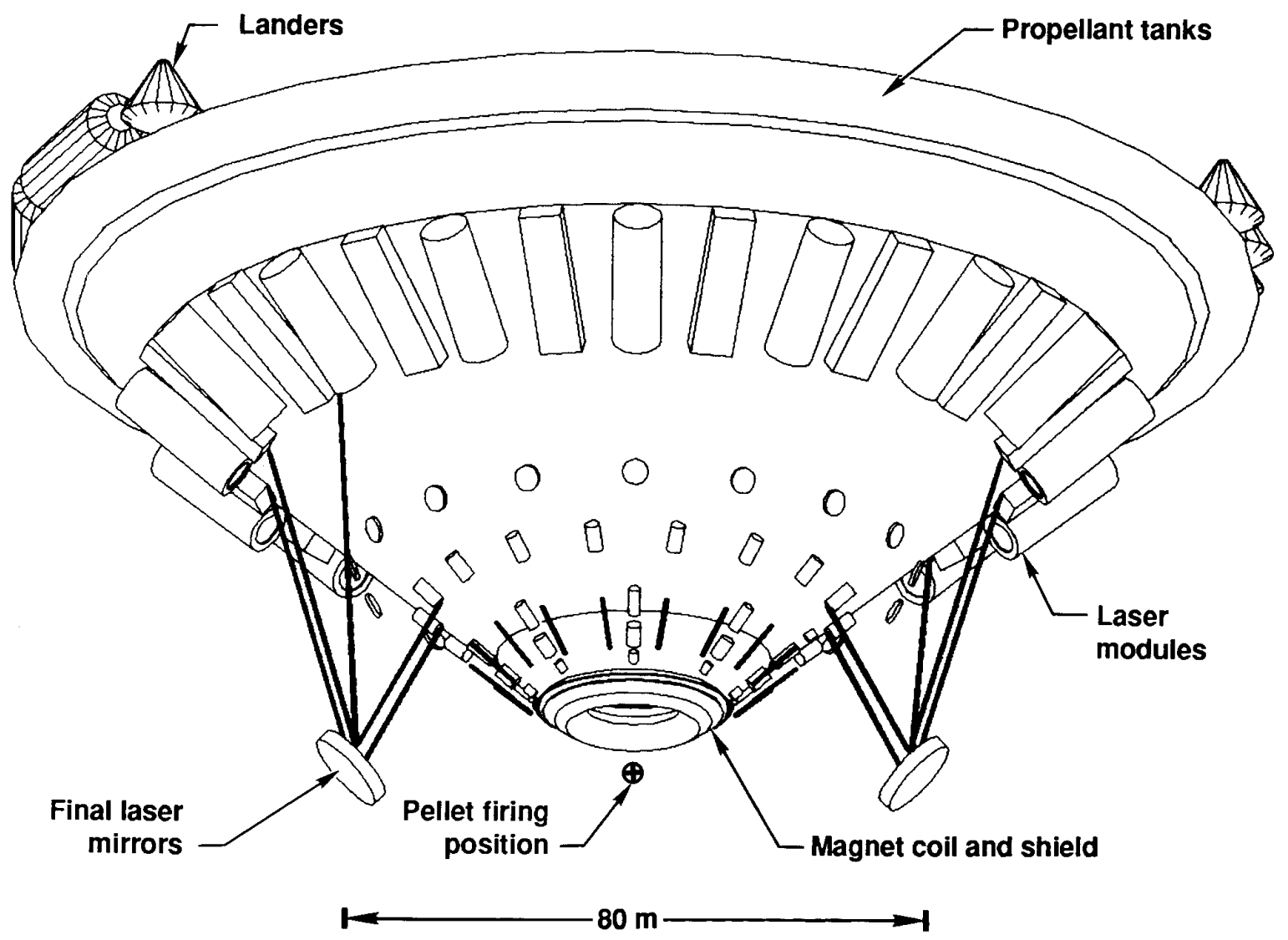

The basic engine operation of VISTA is thus as follows. ICF capsules are injected into the magnetic thrust chamber with the desired repetition rate and with the desired expellant mass per capsule. The capsule is tracked, and imploded using a 5-MJ laser. Depending on the capsule design employed, the released energy is 200 to 1,500 times larger than the laser energy. For the typical energy partition leaving the imploded capsule region, we estimate that one-half of the fusion energy is in the form of high-energy neutrons, one-fourth in $\mathrm{x}$ rays, and one-fourth in charged plasma debris. VISTA uses only the plasma debris because only the charged component is deflectable by the magnet-the rest of the energy is simply discarded. Typically $32 \%$ of the plasma energy is available for jet power as the plasma is redirected by the magnet into an exhaust plume. Consequently, VISTA uses only about $9 \%$ of the fusion output for propulsion. Another 5 to $10 \%$ of the plasma energy is extracted for power generation. As mentioned above, the engine is throttled by changing the capsule repetition rate. Specific impulse, which is set by the plasma temperature, is varied by changing the capsule design or (more simply) by changing the expellant mass per capsule. 
Steerage of the entire spacecraft is accomplished by injection of the ICF capsules slightly off the axis of the magnetic thrust chamber.

\section{I.5 Intent and Overview of This Report}

This report describes the results of our systems analysis performed in 1986-1987, plus the needed extensions and updates to enable the report to be complete enough to be foundational in establishing a realistic concept using fusion-powered space propulsion. The report is not meant to be exhaustive, and we have identified in the text where further studies could be beneficial to validate certain scientific and/or engineering principles.

We first acquaint the reader with ICF and its use in the VISTA concept. We describe the power conversion and conditioning systems needed to operate the laser driver and auxiliary equipment. For power conversion, we consider both a Rankine thermal cycle and Hyde's inductor pickup coil system. Radiators based on heat-pipe technology remove the waste heat from the driver and power systems. One notable aspect of the conical design is that the radiators (with appropriate mechanical stiffeners) actually form the conical surface.

We describe the capsule drivers currently under development for terrestrial ICF applications, and explain the areas where further development is required before applications to an inertial fusion rocket (IFR) are possible. We consider some advanced fusion fuels (DD and $\mathrm{D}^{3} \mathrm{He}$ ), and show that it is not necessary to pursue a technological development path for an IFR that is different from the terrestrial ICF program because the advanced fuels do not offer performance exceeding that for DT until extremely advanced technological levels are attained. However, there are advantages to using the advanced fuels, namely reduced tritium acquisition costs, reduced tritium hazards, and reduced hazards to personnel in other (nearby) spacecraft because of reduced neutron emissions. Therefore, because VISTA is capable of using any of these fuels, the development plan would begin with DT as the best choice for near-term missions, but would switch to DD for the intermediate term, and to DD or perhaps $\mathrm{D}^{3} \mathrm{He}$ in the far term (provided an economical source of ${ }^{3} \mathrm{He}$ can be developed).

With advanced DT technology, piloted-Mars missions can be conducted with a total duration of about 145 days, including a stay on the planet of about 10 days. Roundtrip missions to Pluto will require a little more than 7 years, and roundtrip missions 2.5 times further to $100 \mathrm{AU}\left(1 \mathrm{AU}=\right.$ sun-Earth distance $\left.=1.496 \times 10^{11} \mathrm{~m}\right)$ will require nearly 19 years. In essence, for roundtrips, VISTA reaches coast speeds of 50 to $60 \mathrm{~km} / \mathrm{s}$ for any destination within the interplanetary system.

VISTA was not designed to go to more distant objects, but if it did, peak speeds would still not exceed about $100 \mathrm{~km} / \mathrm{s}$. A roundtrip to 17,225 AU (the Oort Cloud) would therefore take over 1,630 years! A roundtrip to the nearest star, Alpha Centauri (4 light years away, or about 250,000 AU), would take over 12,000 years! Although this performance is considerably better than that possible with either chemical, nuclear-electric (fission), or even antimatter propulsion systems, it is rather clear that VISTA cannot be used to visit the stars and return within a human lifetime. It is possible, however, to go one-way to $10^{4} \mathrm{AU}$ in 20 years, with extremely advanced technology (see Sec. XV.2). 


\section{I.6 Comments on the Reality of ICF}

It should be noted that we emphasize reality in this report. Because of the excitement associated with space travel, many investigators have often introduced concepts for space vehicles and power systems that seem to have attractive features, but the concepts are found to suffer from one or more serious oversights after careful investigation is carried out years later. We take the opposite approach in this report, and describe concepts that have withstood a complete systems analysis. We therefore offer VISTA as a concept that we believe is truly realistic. Our approach is significantly different than that taken by authors who suggest concepts based only on conceptual ideas and/or trajectory analyses (e.g., the "rocket" equation).

Similarly, we use ICF based on the reality of the phenomena as proved both in the explosion of thermonuclear weapons and in experiments in which underground nuclear explosions in Nevada were used to generate $x$ rays to drive ICF capsules. In particular, although it is not well known, Halite/Centurion experiments conducted at the U. S. Nevada Test Site using energy from underground explosions to implode an inertial fusion capsule have already allowed the demonstration of excellent performance, putting to rest the fundamental questions about the basic feasibility to achieve high gain. In fact, the good performance in this series provides a new, higher level of confidence that it will indeed be possible to design working laboratory capsules, such as those for the U. S. National Ignition Facility (NIF).

It is therefore a misconception to believe that it might take decades to experimentally demonstrate that ICF can serve as a source of power for space propulsion. This and other misconceptions have led to many statements that have been published about ICF that are simply not true today-for example, that capsule gains that would be large enough are " 4 orders of magnitude beyond today's capability,"18 that "an order of magnitude in specific mass radiator performance is necessary," and that ICF concepts employing DT "will be unacceptable for long duration missions because of tritium's short 12.3 year half life."19

In addition, when people hear of fusion concepts, they usually think of magnetically confined fusion (MCF) and not ICF, presumably because of ICF's past veil of secrecy and the predominance in funding for MCF during past decades. However, as we stated above, ICF is already known to work with sufficient energy input. This statement cannot be made for MCF or any other advanced propulsion concept except those based on fission. This does not mean that ICF is already fully developed - the truth is quite the contrary-but it does mean that proof of principle for advanced technologies has been demonstrated only for ICF. Thus, the view that ICF is merely a backup for $\mathrm{MCF},{ }^{20}$ although perhaps politically expedient at the time the statement was made, is simply not a realistic perspective.

Consequently, the focus of terrestrial ICF efforts directed towards power production is in reality not a proof of the viability of ICF in principle but a way to improve the economics of fusion power plants-that is, to determine whether ICF will be able to generate power more cheaply than advanced fission reactors or coal/natural-gas-burning plants so that utility companies will want to invest large amounts of capital to develop commercial fusion power. Terrestrial research is therefore attempting to determine the minimum driver energy required with laboratory drivers, because the cost of electricity decreases with the size of the fusion driver that initiates the fusion reactions. This effort to operate near the ignition threshold places additional technical challenges on the field that are not directly relevant for VISTA, which operates well above any ignition threshold. Nevertheless, these terrestrial efforts are important for VISTA for two reasons: (1) greater performance in terms of higher capsule energy gain is required for really spectacular interplanetary transit 
times, and the methods to achieve such gains can presumably be developed at the lower driver energies applicable for terrestrial use; and (2) the best mission performance for space applications does indeed require the smallest overall driver mass, because this reduces the so-called "dry mass" of the spacecraft. For these and other reasons, most terrestrial ICF R\&D will automatically apply for space applications, but space applications will probably require even higher capsule gains and even lower driver masses than terrestrial ICF R\&D may want to pursue. Thus, to be able to use an IFR in the future, interest must be generated now so that development plans can be formulated. Chapter XIV details our discussion of the technology readiness for VISTA.

Another item of reality concerns advanced fusion fuels. The emphasis on the use of $\mathrm{D}^{3} \mathrm{He}$ fuel in some literature is in fact motivated by MCF requirements for high directthrust efficiency and the desire to reduce neutron shielding and radiator masses. ${ }^{21}$ On the other hand, ICF can use DT or DD or D ${ }^{3} \mathrm{He}$ fuels, although with increasingly more advanced requirements for capsule technology. Moreover, the use of matter-antimatter annihilation as a power source in the early $21^{\text {st }}$ century is, at least as seen at this time, beyond the limits of practicality. This is not to say that these other "advanced-technology" systems might somehow be developed at some future date, but only that they are not viable for the near term-and ICF is.

Unless otherwise specified, we will use units as follows: energy in MJ, time in seconds, power in MW, length in meters, and mass in metric tons (one metric ton $=10^{6}$ grams $=1$ tonne $=1 \mathrm{t}$ ). 


\section{THE NATURE OF INERTIAL CONFINEMENT FUSION (ICF)}

To describe the nature of fusion and how it can be applied to space propulsion, it is helpful to begin with a description of fusion in the context of terrestrial fusion power-plant development. It is in this context that many people were introduced to fusion in the past. Unfortunately, because of the limited information that was available at that time, many false notions arose concerning the readiness of the technology. The context we have chosen will help clear up these misconceptions.

\section{II.1 What is fusion?}

Fusion is the nuclear process by which nuclei lighter than iron (e.g., isotopes of hydrogen) are forced to combine (fuse) to form heavier nuclei (such as helium) through a process that releases fast neutrons or protons. Fission, in contrast, is the nuclear process by which the nuclei of elements heavier than iron (e.g., uranium) are bombarded with slow neutrons to produce unstable isotopes, which then decay (break up) into lighter nuclei through the process of radioactivity. Both fusion and fission release energy through the well-known Einstein equation $\mathrm{E}=\mathrm{mc}^{2}$, because the combined mass of the products is less than the sum of the masses of the starting nuclei-hence, mass has been converted into energy.

One difference between fusion and fission is that fusion releases about four times more energy per unit mass, so fusion is preferred from an energetics standpoint. Fusion also involves less radioactivity because, unlike fission, the process itself is not inherently radioactive, and any induced radioactivity (e.g., from neutron emissions activating nearby structures) can be kept to a minimum by the use of so-called low-activation materials. Fusion is also not subject to explosion (as at Chernobyl). Fusion is also not subject to the "China syndrome" (where the uranium fission fuel can melt down through its containment if emergency cooling fails) because light elements do not have the high heat contents typical of the heavy elements used in fission reactors. In addition, light elements have lower decay heats than the fission products produced in fission reactors. Fusion thus offers many advantages over fission.

The drawback for fusion has been the difficulty in developing the technology. Because the nuclei that are fused together in a fusion reaction are all positively charged, high temperatures (about $10 \mathrm{keV}$, or about 100 million degrees Kelvin) are required to overcome what is called the Coulomb (repulsive) barrier and get the nuclei close enough so that they can "stick" (fuse) together. Such high temperatures are achieved by heating the fuel in some way (e.g., by compression) so that the product of fuel density and the confinement time nt can exceed $10^{14} \mathrm{~s} / \mathrm{cm}^{3}$ (the Lawson criterion). Only then will the charged byproducts of the reactions deposit their energies in the fuel and "bootstrap" the temperature up to the required level. At such high temperatures, the fusion fuel cannot be contained by ordinary mechanical vessels, which would melt, so the fuel is confined either magnetically or inertially. Magnetic confinement (such as in a Tokamak) involves a lowdensity gaseous fuel (plasma) confined by heavy external magnets $\left(\mathrm{n} \sim 10^{14} / \mathrm{cm}^{3}\right)$, so seconds or more of confinement are needed to satisfy the Lawson criterion; inertial confinement involves densities nearly a thousand times liquid hydrogen densities (i.e., 200 to $300 \mathrm{~g} / \mathrm{cm}^{3}$ or $\mathrm{n} \sim 6 \times 10^{25} / \mathrm{cm}^{3}$ ), so it requires confinement times of $\sim 2 \times 10^{-12}$ second. The different types of fusion are therefore designated by the type of confinement: inertial confinement fusion (ICF), which is also called inertial fusion, and magnet confinement 
fusion (MCF). Fusion is also known to occur in the center of the sun, where gravitation provides the confinement and the high temperatures.

\section{II.2 What is inertial fusion?}

For inertial fusion, a capsule is prepared that is essentially a spherical "fuel" shell of hydrogen isotopes surrounded by what is called an ablator (i.e., a layer that can be burned away). Such a capsule is irradiated with beams of energy from a "driver." If the irradiation is directly on the capsule, the type of drive is said to be "direct drive." If the driver beams are focused into a small metallic cylinder (a "hohlraum" [German: empty room]) that heats up and irradiates the capsule with $x$ rays, the type of drive is said to be "indirect drive," and the hohlraum with the capsule mounted inside are said to be the "target."

The driver beams (or the $\mathrm{x}$ rays) heat up the surface of the ablator to such an enormous temperature that the surface material blows off radially at great speed. Because for every (momentum) action there is a (momentum) reaction, the blow off causes an inward compression (implosion) of the capsule. As the capsule implodes, the density and temperature at its center increase to the point at which the fusion reactions begin (i.e., the fuel "ignites"). At this time, the inward-moving fuel is confined by its own inertia, because the inward-moving fuel tends to continue moving inward. This explains why this type of fusion goes by the name inertial confinement fusion (ICF).

As the fusion fuel "burns," the nuclei fuse together and release energy in the form of $x$ rays and fast (14-MeV) neutrons as well as particulate kinetic energy (the energy of the vaporized target materials) that is referred to as plasma debris. This sudden release of energy stops the implosion, and reverses it to form a miniature explosion that blows the material apart. This disassembly of the fuel limits the fusion burn, as does the depletion of the $\mathrm{D}$ and $\mathrm{T}$ constituents as they are consumed, so that typically only about $1 / 3$ of the fusion fuel actually fuses (i.e., the burn efficiency is $\sim 1 / 3$ ).

For a terrestrial power-plant application, an ICF capsule is about the size of a marble, and its energy release is about $1 / 4$ ton of TNT equivalent. Such explosion sequences are repeated at the rate of about 5 to 10 targets every second. The energy from such explosions is collected as heat in the wall of a spherical fusion chamber that contains the explosions. This heat can be used to operate a power plant with a steam turbine in much the same way as a coal-fired plant. A fusion plant is hence very similar to a regular power plant, except that a fusion plant needs (1) a driver to generate beams of energy to compress the fusion fuel to the required conditions, and (2) a fusion chamber.

It is noteworthy that inertial fusion will work with different fuels. The simplest fusion reaction, and the one which is the easiest to initiate (because it has the highest cross section), is the combination of one deuterium (D) nucleus with one tritium (T) nucleus to make one helium nucleus ( $\alpha$ particle) plus an energetic $(14.1 \mathrm{MeV})$ neutron. This reaction is referred to as DT fusion, and is written as follows:

$$
\mathrm{D}+\mathrm{T}=\mathrm{n}(14.1 \mathrm{MeV})+\alpha(3.5 \mathrm{MeV})
$$

Deuterium is a heavy form of hydrogen (with one extra neutron in its nucleus), and tritium is a heavier form of hydrogen (with two extra neutrons). The DT reaction releases $17.6 \mathrm{MeV}$ per interaction, or 339 MJ per mg of DT (at 100\% burnup). Only about $1 / 4$ of the total energy released appears as kinetic energy of charged particles (see Sec. III.6). Although tritium is somewhat radioactive, and hence DT fuel is somewhat radioactive, the next generation of inertial fusion could use just deuterium (DD). This type of fuel has about the 
same energy output, is not radioactive, and can be extracted from (sea) water at the rate of about 1 / 15 gram per gallon (i.e., enough for 1 or 2 capsules); one gallon hence has the releasable energy content of 1.8 tons of TNT equivalent (at $1 / 3$ burn-up). The vastness of the oceans therefore provides an essentially inexhaustible supply of fusion fuel. The disadvantage of DD fuel is that it is harder to ignite, requiring about 3 times the ignition temperature (and hence a larger driver, and a different target design).There are also more advanced forms of fusion, such as $\mathrm{D}^{3} \mathrm{He}$ (cf. Sec. III.7).

The advantages of fusion (and ICF in particular) arise from its huge release of energy. It is this high specific energy output that enables ICF to have power/mass ratios from ten to several hundred watts per gram. The huge energy release enables the fuel and a surrounding mass of expellant to reach very high temperatures (typically near $1 \mathrm{keV}$, or $11,600,000 \mathrm{~K}$ ). These high temperatures produce particle speeds of typically $3 \times 10^{5} \mathrm{~m} / \mathrm{s}$, and hence specific impulses in the tens of thousands of seconds. No other viable technology can produce both high power/mass ratios and high specific impulses. For example, a high power/mass fission rocket may only produce a specific impulse of one to two thousand seconds.

In DT fusion Eq. (1), $80 \%$ (14.1 MeV) of the total energy release $(17.6 \mathrm{MeV})$ resides in the neutrons, upon their creation. The remaining energy $(3.5 \mathrm{MeV})$ resides in the alpha particles $\left({ }^{4} \mathrm{He}\right.$ nuclei). The ranges of the alpha particles are generally shorter than the size of the compressed capsule, so all alpha energy is deposited in the fuel. This deposition "bootstraps" the fuel to higher temperatures and higher fusion rates. Most, but not all, of the neutrons escape. In fact, assuming that the column density of a compressed fuel capsule must be at least a few $\mathrm{g} / \mathrm{cm}^{2}$, only about 60 to $70 \%$ of the total energy escapes the capsule in neutron energy. More neutrons are captured when expellant is added, especially hydrogen, which has a short neutron interaction mean free path $\left(4.0 \mathrm{~g} / \mathrm{cm}^{2}\right)$. Perhaps only 50 to $55 \%$ of the total energy will then escape from the capsule-expellant region. A small fraction $(4 \%)$ of these neutrons strike VISTA's magnet coil shield and contribute to vehicle waste heat. The rest of the neutrons travel away from the original location of the capsule isotropically out into space at about $1 / 6$ the speed of light.

The other half of the fusion energy is divided between $x$-rays and the plasma debris (cf. Sec. III.6). The x-rays are of course radiated away in the same manner as the neutrons, while the plasma thermal energy is converted into radial kinetic energy of the expellant ions as the expellant expands from the vicinity of the hot capsule. Put simply, the capsule microexplosion heats the expellant, and all of the mass expands, emitting $x$ rays in the process. By the time the debris has expanded more than one meter, its internal temperature is near $1 \mathrm{eV}$ (i.e., cold), so it stops radiating. The debris radial kinetic energy is still near $1 / 2$ to $1 \mathrm{keV}$, however, even though the plasma temperature is cold. Very crudely, if $E$ is the energy trapped in a debris mass $m$, then the final average debris velocity is approximately $(2 E / m)^{1 / 2}$. It is this high velocity, which basically reflects the original material temperature prior to expansion, that allows VISTA to have high specific impulse.

\section{II.3 The Advantages of ICF}

At this time, ICF has many advantages over MCF. First, ICF is now known to work with sufficient energy input, as explained in Section I.6. Basic scientific feasibility is therefore no longer an issue in principle for ICF, with sufficient energy input.

Second, DT fusion produces fast neutrons whose energy content can be collected-and the environment (e.g., crew quarters) shielded from induced 
radioactivity - only by the introduction of shielding. For ICF, this shielding is minimal because the source of the neutrons is essentially a point (the center of the imploded capsule). The shielding for a terrestrial plant can therefore be placed around the exterior wall of the fusion chamber as a spherical shell, and can even be designed to serve both as shielding and as (or in conjunction with) an absorber of neutron energy for the transfer of heat energy to a heat exchanger. For MCF, however, the source of the neutrons is a large extended source (e.g., the entire doughnut-shaped interior cavity of a tokamak). Consequently, the geometry of the shielding for MCF cannot be a spherical shell, so considerably more shielding is required. Third, because of the low-density nature of the MCF fuel, and the large amount of shielding required, a power plant utilizing MCF is much larger and hence more expensive (and more massive) than we think a power plant utilizing ICF will be.

Fourth, ICF has greater physical separability between the location of the fusion reactions and the emplacement of the high-technology equipment that energizes the fusion fuel. Fifth, ICF offers a clearer approach to maintenance-continuous beam maintenance may even be feasible by including extra driver beams. Sixth, ICF offers more control, because one driver can feed multiple fusion chambers, and variable repetition rate can be employed. Seventh, ICF has an attractive and shorter development path at lower cost (see Sec. XIV.3).

\section{II.4 Why don't we have a terrestrial ICF power plant now?}

Before utility companies will become interested in inertial fusion energy (IFE) - that is, to make energy in a power plant based on ICF-we must demonstrate that IFE can be economically feasible. The main problem for IFE (and fusion in general), other than the complex nature of the technology itself, is the great difficulty in designing a power plant that has a low enough construction cost to permit economic feasibility. The reason for this is that an IFE plant includes extra systems that a fossil-fuel plant does not have, namely, a driver and a fusion chamber. These systems are in addition to the thermal (steam-turbine balance-of-plant) equipment that is common to IFE as well as to other types of power plants. Because the fuel costs for IFE should be almost negligible, the combined cost of the driver and the fusion chamber for IFE must be compared with the cost of the boiler in a fossil-fuel plant and the operational cost of securing fossil fuel. Such a comparison indicates that IFE must demonstrate economic feasibility by designing a driver and a fusion chamber that are low enough in cost.

The size, and therefore the cost, of a fusion chamber is dependent primarily on the plant's thermal power, and only weakly dependent on the energy that the driver beams must focus on the target. In contrast, the cost of the driver is highly dependent on the delivered energy (cost $\sim \mathrm{E}_{\mathrm{dr}}{ }^{1 / 3}$ ). Thus, for a given size of power plant, the economic competitiveness of IFE depends on whether the technology will function reliably at the minimum driver energy. The amount of driver energy required is in turn determined by the target gain (i.e., the ratio of the energy produced by the target and the energy delivered to the target by the driver beams). One key to the economic feasibility of IFE is therefore the ability to operate ICF as close to its ignition threshold as is possible and still maintain reliability. Such operation introduces significant technological challenges in finding the target design that will produce a high-enough energy gain $(>100)$ at a small-enough driver energy ( 1 to 2 megajoules) when target gain tends to increase for a given type of target roughly as the $1 / 3$ power of the driver energy $\left(G \sim E_{d r}^{1 / 3}\right)$. Said differently, we can achieve a larger target gain if we use a larger driver, but economics (and dry-mass constraints for space applications) require minimum driver size. 
The lack of sufficient target gain has made another issue become a key to the economic feasibility of IFE. This secondary key is the scientific feasibility of using a laboratory driver to energize an available type of target. Targets requiring more energy require a larger driver, which is more difficult to develop because of the technological constraints in generating more energy in temporally shaped spatially smooth beams and in delivering this energy efficiently to a target. Because the most reliable target designs deliver calculated gains significantly less than 100 for small driver energies, a larger ( $\sim 2$ megajoule) driver had to be developed. It is the difficulties with the technological challenges dealing with target gain and the feasibility of the required driver that have constrained the early development of IFE.

\section{II.5 Why Consider Fusion for Space Propulsion Systems?}

We have seen that ICF as used by VISTA uses only about $1 / 4$ of the emitted fusion energy, discarding $3 / 4$ of the energy in the form of neutrons and $x$ rays and utilizing only the charged debris plasma in the magnetic thrust chamber. This inefficiency of $1 / 4$ would appear to exactly cancel fusion's inherent advantage of a factor of 4 relative to fission in converting mass into energy by $\mathrm{E}=\mathrm{mc}^{2}$ (see Table II-1). Fission can of course be used in the near term because it is a developed technology and fusion is not, but once fusion is developed, what then? Would fission and fusion be on an equal footing for space applications? No, they would not, because fusion produces high-quality emissions that are directly usable in high mass-flow-rate propulsion systems, and fission and antimatterannihilation concepts do not. Let's examine why this is so.

In practice, one must be able to use the energy emitted by the process being considered, and it is here that fusion is far better than any of the other power sources, including antimatter-matter annihilation. The reason for this is that, at high exhaust-mass flow rates, all of the other power sources require containment vessels to convert the emitted particle energy into a useable form to produce thrust in one direction, and the material temperature constraints to avoid melting the containment vessel constrain either the power/mass ratio or the specific impulse or both.

Chemical reactions, although they afford high power/mass ratios (101 to $\left.10^{3} \mathrm{~W} / \mathrm{g}\right)$, occur at inherently "low" temperatures, thereby producing low specific impulses (less than about $10^{3} \mathrm{~s}$ ). Fission reactions release high-energy neutrons and gamma rays, but they must travel through centimeters of material before interacting and delivering their energies to produce heat; the fission rocket thereby has high power/mass ratios $\left(10^{1}\right.$ to $\left.10^{2} \mathrm{~W} / \mathrm{g}\right)$ but only moderate specific impulses (less than several thousand seconds), while nuclearelectric propulsion (NEP) systems have high specific impulses ( $10^{3}$ to $10^{5} \mathrm{~s}$ ) but only moderate power/mass ratios (less than $1 \mathrm{~W} / \mathrm{g}$ ). Fusion, on the other hand, emits a highspeed debris plasma that is directable with a magnetic field, thereby allowing the inherently high $(\sim 1 \mathrm{keV})$ temperatures of the emitted particles to be directly converted into exhaust velocities without changing the particle speeds. Antimatter annihilation has such long-range reaction products that the use of appropriate expellant is impractical without exceeding the dynamical limits of the system (magnet) that redirects the products into an exhaust. Thus, only fusion allows both high power/mass ratios (101 to $\left.10^{3} \mathrm{~W} / \mathrm{g}\right)$ and high specific impulses $\left(10^{3}\right.$ to $\left.10^{6} \mathrm{~s}\right)$. 
Table II-1 Attributes of Various Power Sources

\begin{tabular}{|l|c|l|l|}
\hline \multicolumn{1}{|c|}{ Power Source } & $\begin{array}{c}\text { Total Efficiency For } \\
\text { Converting Mass } \\
\text { Into Energy }\end{array}$ & Emitted Particle & Range in Materials \\
\hline \hline Chemical reactions & Approx. 10-10 & Light & Angstroms \\
\hline Fission reactions & 0.0009 & $\begin{array}{l}\text { Neutrons } \\
\text { Gamma rays }\end{array}$ & $\begin{array}{l}\mathrm{cm} \\
\mathrm{cm}\end{array}$ \\
\hline Fusion reactions & 0.0038 & $\begin{array}{l}\text { X rays } \\
\text { Neutrons } \\
\text { Debris plasma }\end{array}$ & $\begin{array}{l}\mu \mathrm{m} \\
\mathrm{cm} \\
\text { Directable with a } \\
\text { magnet }\end{array}$ \\
\hline $\begin{array}{l}\text { Antimatter-matter } \\
\text { annihilation }\end{array}$ & 1.000 & $\begin{array}{l}\text { Pions } \\
\text { Gamma rays }\end{array}$ & $\begin{array}{l}\mathrm{cm} \\
\mathrm{cm}\end{array}$ \\
\hline
\end{tabular}

ICF is even expected to outperform MCF if ICF can achieve a low driver mass because (1) MCF uses a large volume of fuel, unlike ICF's point source, so large shielding masses are required to avoid neutron irradiation of spacecraft components and the crew; and (2) MCF incorporates high magnet masses at high engine power levels. Thus, it is a good guess that ICF systems $(10-20 \mathrm{~W} / \mathrm{g})$ will probably outperform the MCF systems $(1-10 \mathrm{~W} / \mathrm{g}) .^{22}$ Consequently, an ICF-powered spacecraft like VISTA is probably the fusion configuration with the best performance of any fusion system, and certainly the best system among practical non-fusion configurations for $21^{\text {st }}$-century interplanetary missions. Some of the reasons why NASA could consider fusion for its advanced planning have already been documented. ${ }^{23}$ Other MCF and ICF comparisons are described in section XVI.

\section{II.6 Advanced ICF target concepts}

We assume that capsule gains of 300 to 2000 must arise through advanced highly speculative target concepts such as the fast ignitor. ${ }^{24}$ This concept employs capsule compression less sophisticated than that for a normal target, followed by a $\sim 200$-ps $10^{18}$ $\mathrm{W} / \mathrm{cm}^{2}$ laser pulse to burn through the ablated material vaporized by the compressional driver beams and create a "channel" to the fuel core. Ignition is accomplished with a 30-ps $10^{20} \mathrm{~W} / \mathrm{cm}^{2}$ laser pulse directed through the channel. Such a concept holds the promise of target gains in the 500 to 1000 range, and perhaps higher, because less energy is used in compression of capsule material. Other concepts may be required to get gains well above 1000 . 


\section{TARGET SYSTEMS}

The term target refers to the entire laser target assembly including the capsule itself, the hohlraum (if present), and any surrounding expellant mass. We use the term target and not "pellet" because the whole assembly is specifically the target that a laser driver must "hit." The term capsule refers only to the spherical combination of the fusion fuel and other layers integrally attached to the fuel layer. The term propellant includes the fusion fuel (DT) and other target fluids (including the expellant placed around the hohlraum).

\section{III.1 Capsule Design}

The optimum design of a target, as we envision it for VISTA, includes a capsule driven indirectly with $x$ rays-that is, the target includes a high-gain capsule placed inside a hohlraum, which is in turn placed inside the expellant. Laser beams directed into the hohlraum through holes in the expellant create $x$ rays, which implode the capsule through intense vaporization (ablation) of its outer surface (that's why the outer capsule layer is called the ablator). A generic type of hohlraum target thus looks like the following:

\section{Figure III-1: A Generic Type of Target for Indirect Drive}

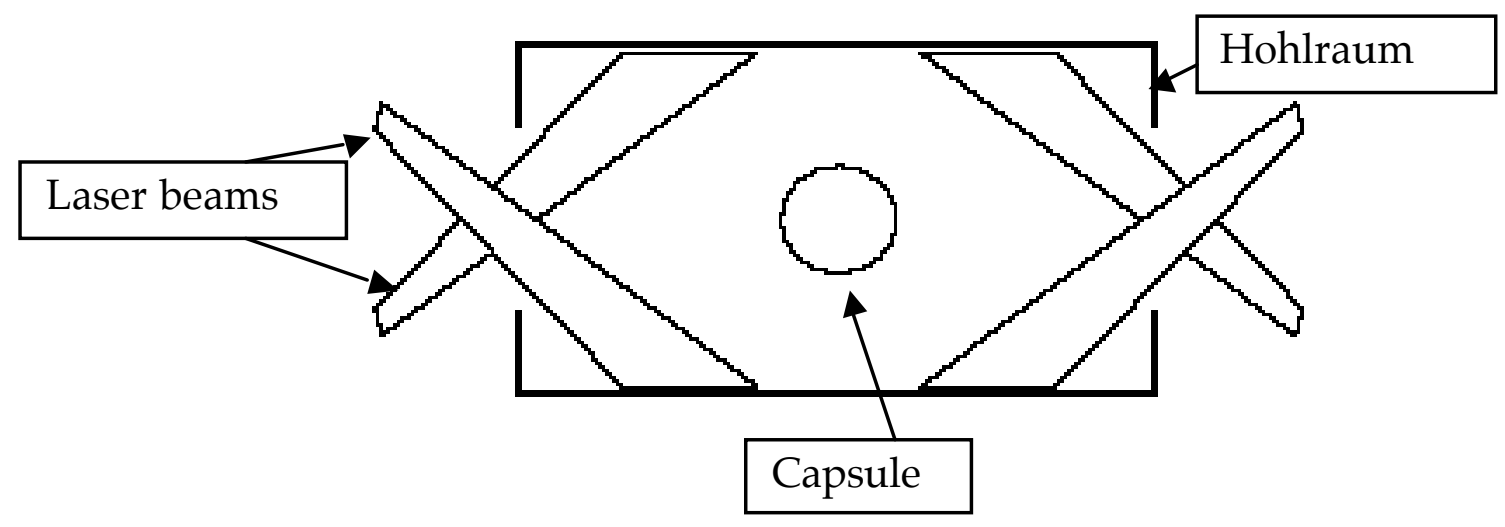

Indirect drive is preferred over direct drive for VISTA application because direct drive would require symmetrical illumination of a capsule, and hence many final-optic mirrors - not just the one or two needed for indirect drive as shown in Fig. I-1.

Because it is important for target assembly, handling, and tankage to have some idea of the overall concepts in ICF capsule design, we describe here some of the general features of capsules that might be used for VISTA. The canonical design of a high-gain capsule is composed of a cryogenic shell of DT fuel surrounded by some sort of ablator material, with a small amount of DT gas in the center. The DT fuel and the ablator material are both in their cryogenic states wicked into foam substrates or equivalent. ${ }^{25}$ The ablator material can in fact be hydrogen seeded with higher-Z contaminants. The amount of gas in the central volume depends on the equilibrium vapor pressure of the DT at the fielded temperature, and is therefore variable. This gas pocket serves as an ignitor for the capsule, because when the capsule is imploded, the DT gas is the first fuel to ignite. A burn front can then propagate throughout the remaining fuel region. 
Operation of a target is as follows. First, $x$ rays created inside the hohlraum by the laser beams ablate the out capsule layer. This ablation creates an inward force that implodes the fuel shell and compresses the central gas volume. The gas, being of lower density, reaches a much higher temperature than the fuel shell. The higher temperature indicates a greater heat content per unit mass, but because there is so little gas, the total energy concentrated in the gas is very small. Nevertheless, the higher gas temperature causes a much higher fusion rate, so the gas serves as an ignitor (hotspot) for the main fuel. By concentrating the compressional energy in the gas and not in the main fuel, a smaller laser system is required for the same fusion yield, thus permitting a more efficient implosion (i.e., higher gain: see Sec. III.5).

The maximum capsule output in VISTA is 7,500 MJ, assuming an arbitrary capsule gain of 1,500 with a 5-MJ driver (cf. Sec. III.5, Eq. 4). This release is equivalent to that of 1.8 tons of TNT, which is about eight times larger than that from the capsule envisioned for terrestrial ICF power plants. Because DT fusion releases $339 \mathrm{MJ} / \mathrm{mg}$, about $66 \mathrm{mg}$ of DT are needed to produce 7,500 MJ of fusion output if the fusion burn efficiency is $33 \%$, but only 46 $\mathrm{mg}$ of DT is required for a burn efficiency of $47.6 \%$ (which is assumed here for a compressed fuel column density of $5 \mathrm{~g} / \mathrm{cm}^{2}$ ). Because DT is $60 \%$ tritium by mass, each capsule therefore contains at least $28 \mathrm{mg}$ of tritium. Peak engine operation at $30 \mathrm{~Hz}$ will thus consume at least $120 \mathrm{~kg} /$ day of DT and $72 \mathrm{~kg} /$ day of tritium. The fuel and hohlraum masses, however, are essentially negligible in comparison with the typical expellant mass of 50 grams per target.

\section{III.2 Expellant}

Hydrogen has the highest probability for scattering a fusion neutron to absorb energy and increase the debris kinetic energy (and hence the specific impulse). The mean free path for neutron energy attenuation in hydrogen is only $4.0 \mathrm{~g} / \mathrm{cm}^{2}$. Hydrogen can also be stored, transported, and loaded in a capsule all in its liquid state. A cryogenic expellant can, in fact, be held firmly by capillary forces in spherical shells of plastic foam. ${ }^{26}$ Liquid hydrogen expellant also permits changing the mass of expellant for each target during the mission, if the need should arise.

We shall therefore assume that the expellant is hydrogen. Nevertheless, hydrogen has disadvantages. For example, its low density of $0.077 \mathrm{~g} / \mathrm{cm}^{3}$ causes the targets to have a very large size, which complicates the design of the target injection system. In addition, hydrogen debris plasma tends to be less conductive than higher- $Z$ expellants, thus exacerbating plasma decoupling in the plume, which can lower jet efficiency (see Sec. V.5). Moreover, tankage is at least twice as large for a fluid than for a solid, and it may be difficult to configure a liquid so it has holes for the driver beams to pass through to reach the hohlraum.

We, therefore, expect that it is quite likely that the expellant will have to be moderate$Z$ to high-Z, especially to obtain maximal jet efficiency. For VISTA, most any type of expellant can be used, if it can be stored, and later loaded around a target. If solid expellants are considered, we suggest merely using more massive hohlraums.

A more advanced consideration is whether the expellant should be spatially shaped to reduced neutron emissions in particular directions (e.g., toward spacecraft components, in order to reduce the need for neutron shielding). Although we left evaluation of this subject 
to a future study, Sahin et al. ${ }^{27}$ have since verified that such spatial shaping can reduce neutron irradiation of spacecraft components by factors up to about 12.5 .

\section{III.3 Target Fabrication and Assembly}

We envision that all of the hohlraums and capsules for a mission will have been prefabricated and pre-assembled prior to the mission, and stored onboard without their fillings of cryogenic fluids. This need not be the case, however, because fabrication in a onboard target factory is not only possible, but may have its advantages. For example, onboard fabrication could improve sphericity due to the long periods of reduced gravity (e.g., near the rotation axis), and would eliminate shipping and storage damage to the capsule assemblies. A foam substrate, if used for hydrogen expellant, will most likely be manufactured onboard due to the otherwise large volume required for capsule storage (cf. Sec. III.8).

Because the use of wetted foams is a relatively new technology, more development is required before final decisions can be made regarding the best place and methods of capsule fabrication. Nevertheless, we envision the use of automated fabrication techniques for all capsule materials patterned after those conceptualized and parameterized for the fabrication and electrostatic transport of laboratory glass-sphere targets. ${ }^{28}$ More research on this topic is definitely in order.

Once any final assembly is completed, if required, the DT fuel, hydrogen ablator, and hydrogen expellant will be wicked into their substrates immediately prior to use. From then on, the capsules must be kept cold (at about $20 \mathrm{~K}$ ). The size of each filled target assembly will be roughly the size of the liquid hydrogen expellant (density $0.077 \mathrm{~g} / \mathrm{cm}^{3}$ ); for a 50-g expellant, the target diameter will be about $12 \mathrm{~cm}$, which is the size of a large grapefruit.

\section{III.4 Target Acceleration, Injection, Tracking, and Beam Pointing}

Detailed analyses have not been conducted for the explicit systems needed for target acceleration, injection, and tracking for an IFR. However, many of the general concepts for a terrestrial fusion power plant should be directly applicable. Some of the problems associated with target injection, tracking, and laser-beam pointing have been discussed by Petzoldt and Monsler. ${ }^{29}$ Differences between the terrestrial and IFR systems result primarily because 1 ) the IFR target (with its expellant) is much larger, and 2) the IFR reaction chamber ambient pressure (and temperature) is zero. The expellant makes it more difficult to cool the fuel and remove the tritium radioactive-decay waste-heat generation of $1 / 3 \mathrm{~W} / \mathrm{g}$. The removal of this waste heat to prevent boiling of the cryogenic liquids during acceleration and injection is in fact the most crucial problem for the target systems. More massive targets are also harder to transport. On the other hand, the lack of an ambient chamber pressure reduces clearing times and target tracking errors for the IFR, and the small solid-angle subtended by spacecraft material as seen near the target-firing point reduces thermal constraints during injection.

The acceleration and injection phases are limited by physical as well as the thermal constraints. First, the minimum injection speed vinject is that which takes the target from the edge to the center of the thrust chamber (i.e., through a distance equal to the radius $r$ of the chamber) in a time interval equal to roughly $\mathrm{kn}^{-1}$, where $\mathrm{n}$ is the IFR repetition rate in $\mathrm{Hz}$ 
and the $\mathrm{k}$ is the fraction of the interpulse period that is available for injection (i.e., the fractional time the chamber is clear of debris and vaporized material from the previous pulse). Typically, $\mathrm{k}$ is about 0.7 and $\mathrm{r}$ is about 15 meters. The minimum injection speed is thus $v_{\text {inject }}=\mathrm{rn} / \mathrm{k}$, or $200-600 \mathrm{~m} / \mathrm{s}$ depending on $\mathrm{n}$. This speed assumes the use of a rotating shutter or similar apparatus to block the target accelerator output port for $1-\mathrm{k}=$ $30 \%$ of the interpulse period, to allow for chamber clearing from the previous pulse, and to protect the target accelerator from debris damage. Target injection speeds normally have an additional constraint arising from the requirement to keep the target cold as it traverses a hot terrestrial reaction chamber (e.g., a minimum of $300 \mathrm{~m} / \mathrm{s}$ may be required for an 800 $\mathrm{K}$ chamber ${ }^{30}$ ). In space, however, no such additional constraint is of great consequence.

A second constraint is that the acceleration of the target to vinject must not exceed $\mathrm{a}_{\max } \mathrm{g}$, where $\mathrm{a}_{\max }$ is the maximum number of gee's the target can withstand and $\mathrm{g}=9.8$ $\mathrm{m} / \mathrm{s}^{2}$ is the acceleration due to gravity. That is, the acceleration time $t_{\text {accel }}$ must be at least $\left(v_{\text {inject }} / a_{\max } g\right)$. For constant target acceleration, the required acceleration distance is thus at least

$$
\mathrm{s}_{\text {accel }}=(1 / 2) \mathrm{a}_{\max } \mathrm{at}_{\text {accel }}{ }^{2}=(\mathrm{rn})^{2} /\left(2 \mathrm{a}_{\max } \mathrm{g}\right) .
$$

Suppose we assume $\mathrm{a}_{\max }=100^{31}$ (wetted foam can withstand acceleration forces of hundreds of gee's or more, due to the strong capillary forces). For a chamber of radius $15 \mathrm{~m}$, the minimum accelerator distance is hence $3 \mathrm{~m}$ at $5 \mathrm{~Hz}$ and $10^{3} \mathrm{~m}$ at $30 \mathrm{~Hz}$. This shows that the spacecraft in Fig. I-1 is large enough to accommodate a target accelerator provided the repetition rate does not exceed a few tens of Hz. Note that the time scale for targets to traverse the accelerator may be much longer than the interval between fusion pulses. This merely requires targets to follow one another through the accelerator spaced at

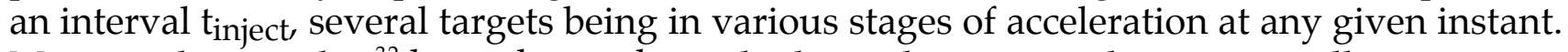
More modern studies ${ }^{32}$ have shown that a dual-membrane capsule support will permit target accelerations well in excess of $10^{3} \mathrm{~m} / \mathrm{s}^{2}$, which would allow smaller acceleration distances and higher repetition rates.

A cryogenic target will most likely not suffer thermal degradation during its transit through the thrust chamber, because the transit time is short and the effective temperature of the environment (looking mostly out into space) is not much above $3 \mathrm{~K}$. On the other hand, the target must be protected during acceleration, so the accelerator channel must be at cryogenic temperatures. In addition, some mechanism must be included to extract the $1 / 3 \mathrm{~W} / \mathrm{g}$ tritium decay heat. The concept generally envisioned to satisfy both of these requirements for terrestrial applications (without expellant) is to encase the target in a thermal shield called a sabot. The sabot is designed with enough thermal capacity to absorb the tritium decay heat, and in addition, to act as the bullet accelerated in a tight-fitting pneumatic barrel that adds rifling (rotation) to the exiting sabot for trajectory stabilization. In our application, such a sabot would have to be removed (by its own centrifugal force) before the target enters the thrust chamber, and be recycled, so that the sabots don't represent a mass loss. Alternately, it may be possible to coat the expellant with a thin metallic layer, and let the expellant act as the sabot (which becomes very easy if the expellant is iron). Perhaps a magnetic-induction accelerator could then be used instead of the pneumatic accelerator. More thermal calculations are needed like those done for terrestrial applications by Pitts ${ }^{33}$ to ensure that $30 \mathrm{mg}$ of tritium can in fact be buried inside $50 \mathrm{~g}$ of hydrogen (or iron) expellant. In addition, special precautions are needed in 
designing the outlet of the target injector, which is in direct line-of-sight to the target and, thus, susceptible to erosion from target emissions. A further complication for the space application is the location of the accelerator channel and / or sabot trajectory on the conical spacecraft frame that itself constitutes radiators operating at high temperatures to expel waste heat. In any case, to avoid unnecessary power consumption, future studies must determine how the capsule can be cooled and placed inside some sort of thermal barrier without cooling a high- $Z$ expellant to cryogenic temperatures.

The acceleration region is followed by a target coast region for several reasons. It is here that sabots are removed, if they are used. A coarse target trajectory is determined here, and modified by electrostatic or inductive means to provide for corrections or vehicle steerage. This trajectory offers an estimate of the position and time of arrival of the target near the center of the thrust chamber, where the laser beams must focus on the holes in the expellant.

General tracking of the target can be accomplished by illumination with a CW laser and use of a tracking telescope to track a bright glint off the target. The best tracker system uses the same optics as the main laser system, and is called a shared-aperture system. ${ }^{34}$ Such a system eliminates relative misalignment errors and vibrational jitter between the tracking and laser-pointing systems. Special features, yet unspecified, might have to be added to the expellant exterior to track the holes in the target, if it proves infeasible to detect the physical holes in the otherwise smooth (metallic) expellant coating. This issue becomes inconsequential, however, if the expellant is added by merely increasing the thickness of the hohlraum wall.

Laser-beam pointing will be accomplished by steering mirrors (deformable "rubber" mirrors are not needed because the chamber volume is not subject to turbulence, thermal boundary layers, ambient-gas breakdown, etc.). Because the final turning mirrors are unshielded against neutron damage, and because they are located in a region exposed to an x-ray fluence of about $0.4 \mathrm{MJ} / \mathrm{m}^{2}$, it might be better to steer the beams at the next upstream optical elements. X-ray protection of the final mirrors themselves can be avoided through use of special surface materials and placement far enough from the target. Use of techniques being designed for terrestrial mirror protection (e.g., a puff of gas in front of the mirrors for every pulse) cannot be employed without significant loss in jet efficiency, as well as degradation in focusability and pointing accuracy (see Sec. IV.3).

\section{III.5 Target Performance (Gain)}

The fusion release from a target is generally stated as the product of the energy delivered by the laser driver (e.g., $5 \mathrm{MJ}$ ) and a quantity called the gain of the target. The gain is defined as the ratio of the fusion energy produced by the target and the energy supplied to the target by the driver. Current DT technology permits target gains estimated to be as large as 50 to 100 (see below), while very advanced technology may permit gains larger than this by factors of at least 10 . According to simple analytic models, ${ }^{35}$ the target gain $G$ for the most optimum target compression is a function of the laser driver energy $E_{\mathrm{dr}}$ (in MJ) and the compressional efficiency $\varepsilon_{\text {comp: }}$

$$
\mathrm{G}=\mathrm{c} \varepsilon_{\mathrm{comp}}\left(\varepsilon_{\mathrm{comp}} \mathrm{E}_{\mathrm{dr}}\right)^{\mathrm{n}}
$$


For the Meyer-Ter-Vehn (MTV) Model, $\mathrm{c}=6,000$ and $\mathrm{n}=0.3$; for the Kidder Model, $\mathrm{c}=$ 30,000 and $n=0.4$. The MTV Model is the more conservative, and indicates gains about a factor of 6 less than the Kidder model. Use of polarized-DT fuel would increase the gain in either model by a factor of (1.5) $0.9=1.4 .^{36}$ Thus, for $\mathrm{E}_{\mathrm{dr}}=5 \mathrm{MJ}$ and maximum compressional efficiencies ( 0.2), G is expected to be between 1200 and 6000. Such high gains are speculative. Nevertheless, as an optimistic gain model based on both MTV and Kidder, we shall assume the following relationship here

$$
\mathrm{G}=877 \mathrm{E}_{\mathrm{dr}}{ }^{1 / 3}
$$

The exponent can vary from 0.3 to 0.4 , while for hydrodynamic efficiencies varying from $5 \%$ to $10 \%$, the leading coefficient can vary from 100 to 300 for an exponent of 0.3 , and from 450 to 1200 for an exponent of 0.4 . We hence chose a leading coefficient of 877 so Eq. (4) yields a gain of 1500 at $5 \mathrm{MJ}$.

Gains as high as those suggested by Eq. (4) are out of the question with near-term technology, but not with advanced technology. Near-term DT gains are better described by $^{37}$

$$
\left.\mathrm{G}=\left(1300 \mathrm{E}_{\mathrm{dr}}-1040\right)\right)^{0.53}
$$

or perhaps ${ }^{38}$

$$
\mathrm{G}=16 \mathrm{E}_{\mathrm{dr}}^{2 / 3}
$$

Equation (5a) indicates a gain of only 96 for 5 MJ. For the fast ignitor (Sec. II.6), the gain for $5 \mathrm{MJ}$ is assumed to be about 760. according to a formula like the following: ${ }^{39}$

$$
\mathrm{G}=400 \mathrm{E}_{\mathrm{dr}}^{0.4}
$$

There is hence a range of technologies that should become available for ICF, manifested in gains from 100 for near-term technologies to about 1000 or more for advanced technologies.

We emphasize that the worth of ICF as a source of engine power is dependent on the magnitude of gain that is achieved in future target development. If the maximum gains achieved are near 100, an IFR will not be competitive with other technologies. If the maximum gains achieved are anywhere near 1000, an IFR will probably outperform other technologies with ease. The target gain is hence the most important development item for an IFR. This is particularly true in light of the current laboratory gain achieved, which is less than 1. The NIF is expected to achieve gains of 10 to 20 for $E_{d r}=1.8 \mathrm{MJ}$. Gains near or above 1,000 thus represent a very large extrapolation from existing laboratory achievement, as well as that expected near ignition threshold on the NIF.

The importance of target gain can be illustrated by examining the theoretical maximum yields (i.e., energy releases) available from targets of various DT fuel masses. Assuming that yield is equal to the product of fuel mass, $3.4 \times 10^{5} \mathrm{MJ} / \mathrm{g}$, and a burn efficiency of about one-third, we obtain the set of yields and gain values shown in Table III1. Target yield is thus an indicator of the amount of fuel mass, but target gain for a given driver input is an indicator of target technology. 
C. Orth, UCRL-LR-110500

Table III-1 Phenomenological Target Gains for Different Fuel Masses

\begin{tabular}{|c|c|c|}
\hline $\begin{array}{c}\text { DT } \\
\text { Fuel Mass } \\
\mathbf{( g )}\end{array}$ & $\begin{array}{c}\text { Maximum Yield } \\
\text { for } \boldsymbol{\varepsilon}_{\text {bum }}=\mathbf{1} / \mathbf{3} \\
(\mathbf{M J})\end{array}$ & $\begin{array}{c}\text { Target Gain } \\
\text { If } \\
\mathbf{E}_{\mathbf{d r}}=\mathbf{5} \mathbf{~ M J}\end{array}$ \\
\hline 0.005 & 570. & 113. \\
\hline 0.01 & $1,130$. & 227. \\
\hline 0.02 & $2,270$. & 450. \\
\hline 0.05 & $5,670$. & $1,130$. \\
\hline 0.1 & $11,300$. & $2,270$. \\
\hline 0.2 & $22,700$. & $4,500$. \\
\hline 0.5 & $56,700$. & $11,300$. \\
\hline
\end{tabular}

\section{III.6 Target Emissions}

When the DT target burns inside the expellant, most of the yield is in the form of 14.1 $\mathrm{MeV}$ neutrons, which begin leaving the target at a speed of about $5 \times 10^{7} \mathrm{~m} / \mathrm{s}$. Some of these neutrons interact in the several $\mathrm{g} / \mathrm{cm}^{2}$ of compressed target material, and some interact in the hydrogen expellant. As a result, only about half of the neutrons leaving the target are still above $10 \mathrm{MeV}$. That is, there is a whole spectrum of neutron energies leaving the target; they are not all still at $14.1 \mathrm{MeV}$. The fraction of the total fusion energy that emerges from the target in the spectrum of neutrons can be approximated by the following formula:

$$
f_{\text {neut }}=0.80 \exp \left[-\left(x_{\text {target }} / 18\right)-\left(x_{\text {expellant }} / 4\right)\right]
$$

where the 0.80 factor is the fractional energy in neutrons at production (i.e., $14.1 \mathrm{MeV} / 17.6$ $\mathrm{MeV}), \mathrm{x}$ is the respective column density in $\mathrm{g} / \mathrm{cm}^{2}, 18 \mathrm{~g} / \mathrm{cm}^{2}$ is the mean free path of neutrons in the burned DT- ${ }^{4} \mathrm{He}$ mixture, and $4 \mathrm{~g} / \mathrm{cm}^{2}$ is the mean free path of neutrons in liquid hydrogen. For example, if the target is $5 \mathrm{~g} / \mathrm{cm}^{2}$ at peak compression and we employ about $1 / 3 \mathrm{~g} / \mathrm{cm}^{2}$ of liquid-hydrogen expellant, then $\mathrm{f}_{\text {neut }}=0.56$. Nevertheless, we emphasize that gamma rays can be produced when the emitted neutrons interact in the target material or the added expellant. We will therefore use $\mathrm{f}_{\text {neut }}$ to represent the total fractional energy in both neutrons and gamma rays as the emissions leave the expellant region. Precise neutronics code calculations are required to obtain more accurate information for any particular case. The energy in the neutron and gamma-ray emissions can then be increased because of exoergic nuclear reactions in the coil shield and/or blanket materials (see Sec. VI.5 for a discussion of the energy multiplication factor).

Although a small burst of high-energy $\mathrm{x}$-rays (above about $100 \mathrm{keV}$ ) is able to penetrate the target material and leave the target essentially instantaneously, the bulk of the x-ray emission occurs after the thermal wave from the hot core has propagated through all of the target and expellant layers to reach the surface of the expanding cloud of expellant. Then a burst of soft x-rays is emitted. This happens very quickly for hydrogen expellant, but occurs rather slowly for lead expellant. The energy not emitted as neutrons 
or x-rays remains in the plasma debris, and ends up as kinetic energy of the debris ions (which are mostly expellant ions, not target ions).

For scaling purposes, it is necessary for us to have relationships for the fractions of the total fusion energy that ends up in the form of $\mathrm{x}$-rays and debris, $\mathrm{f}_{\mathrm{x} \text {-ray }}$ and $\mathrm{f}_{\text {debris, }}$ as we change the mass of the expellant. These fractions will obviously change with the density and mass of the expellant. Unfortunately, however, the complex hydrodynamic phenomena associated with the explosion makes it very difficult to make an analytic calculation of these emission fractions. One might guess that the fractional energies in $\mathrm{x}$ rays and debris are divided roughly equally, with half in x-rays and half in debris, with a spread that varies from $\sim 25 \%$ to $\sim 75 \%$ for the expellant masses and densities of interest here. We therefore chose the following empirical formula to specify the debris fraction:

$$
\mathrm{f}_{\text {debris }}=\left(1-\mathrm{f}_{\text {neut }}\right)\left[0.25+0.50\left(1-\exp \left\{-(1 / 15) \mathrm{m}_{\text {expellant }} \operatorname{sqrt}\left(\rho_{\text {expellant }}\right)\right\}\right)\right]
$$

where the expellant mass $m_{\text {expellant }}$ is in grams and the expellant density $\rho_{\text {expellant }}$ is in $\mathrm{g} / \mathrm{cm}^{3}$. We then have

$$
f_{x-\text { ray }}=1-f_{\text {neut }}-f_{\text {debris }}-f_{\text {loss }}
$$

where $\mathrm{f}_{\text {loss }}$ is the fractional loss in coupling laser energy to $\mathrm{x}$ rays contained by the hohlraum, which we will arbitrarily assume to be of order 7\%. For example, for $50 \mathrm{~g}$ of expellant at density $0.077 \mathrm{~g} / \mathrm{cm}^{3}$ wicked into a foam shell of inner radius $1 \mathrm{~cm}, 56 \%$ of the fusion energy is in neutrons (not $80 \%$ ), $24 \%$ is in charged-particle debris plasma (not 20\%), and $20 \%$ is in x-rays. Thus $24 \%$ of the energy is directable by a magnetic field.

\section{III.7 Advanced Fuels}

The only advanced fuels that we consider here are DD and $\mathrm{D}^{3} \mathrm{He}$. We will not consider $\mathrm{p}^{11} \mathrm{~B}$, or other more exotic fuels because the $\mathrm{E}_{\mathrm{dr}}$ required to achieve ignition is much too large to make these fuels practical for space propulsion unless DT hotspots or other concepts can be employed, all of which is beyond the scope of this report.

\section{III.7.1 DD Fuel}

Complete DD fusion is specified by two primary reactions,

$$
\begin{aligned}
& \mathrm{D}+\mathrm{D}={ }^{3} \mathrm{He}(0.82 \mathrm{MeV})+\mathrm{n}(2.45 \mathrm{MeV}) \\
& \mathrm{D}+\mathrm{D}=\mathrm{T}(1.01 \mathrm{MeV})+\mathrm{p}(3.02 \mathrm{MeV})
\end{aligned}
$$

plus two secondary (catalyzed) reactions,

$$
\begin{gathered}
\mathrm{T}+\mathrm{D}={ }^{4} \mathrm{He}(3.5 \mathrm{MeV})+\mathrm{n}(14.1 \mathrm{MeV}) \\
{ }^{3} \mathrm{He}+\mathrm{D}={ }^{4} \mathrm{He}(3.6 \mathrm{MeV})+\mathrm{p}(14.7 \mathrm{MeV})
\end{gathered}
$$

(The reaction $\mathrm{n}+{ }^{3} \mathrm{He}=\mathrm{p}+\mathrm{T}$ is not expected to be significant.) Assuming the produced $\mathrm{T}$ and ${ }^{3} \mathrm{He}$ also burn, the net result is thus 
That is, per binary DD reaction, $14.4 \mathrm{MeV}$ are released per interaction, or $347 \mathrm{MJ}$ per $\mathrm{mg}$ of DD (assuming 100\% burn up). The energy release per mg is thus comparable to DT fusion $(339 \mathrm{MJ} / \mathrm{mg}$ ). For DD fusion, however, $61.7 \%$ of the total energy ends up in charged particles at each reaction site, instead of the $20 \%$ for DT, and $38.3 \%$ of the total energy is in neutron kinetic energy. That is, the range of a $3 \mathrm{MeV}$ proton in $\mathrm{H}_{2}$ is $0.006 \mathrm{~g} / \mathrm{cm}^{2}$, while the range of a $14.7 \mathrm{MeV}$ proton is $0.108 \mathrm{~g} / \mathrm{cm}^{2}$, so in $\mathrm{DD}$, which has the same number of electrons but twice the mass, the respective ranges ought to be roughly twice these values (because the energy loss is based on the electrons with which the proton would interact). Thus, only the neutrons from the DD reactions escape a target with a compressed column density of 3 to $5 \mathrm{~g} / \mathrm{cm}^{2}$. In fact, even some of the neutrons interact in this amount of material (as per Eq. 6), so the fractional energy into charged-particle debris arising from the heated fuel and expellant actually starts at a value somewhat higher than 61.7\% ( $74 \%)$. Some of this energy (perhaps half) is then radiated from the plasma as $x$ rays. The exact energy remaining in the charged debris plasma (i.e., the amount redirectable by a magnetic thrust chamber) depends on the target design and the amount of expellant around the capsule. As a rough approximation, we will use Eqs. (6) and (7) here, which indicate that $\sim 26 \%$ of the total energy emerges as neutron kinetic energy, $\sim 37 \%$ emerges as $x$ rays, and $\sim 37 \%$ emerges as charged debris plasma kinetic energy, the latter being only 1.5 times the fraction attainable from DT.

It is interesting to note that the DD cross sections for Eq. (9) are one and one-half decades smaller at a burn temperature of $100 \mathrm{keV}$ than the cross section for DT, and about one decade smaller than that for $\mathrm{D}^{3} \mathrm{He}$. The DT cross section peaks at $\sim 70 \mathrm{keV}$ and the $\mathrm{D}^{3} \mathrm{He}$ cross section peaks at $\sim 300 \mathrm{keV}$, but the DD cross section peaks well above $1 \mathrm{MeV}$. Consequently, DD fuel burns only weakly at ICF scales, because the burn temperature is typically less than $100 \mathrm{keV}$. The DD legs (Eq. 9) thus burn sluggishly, then when some T or ${ }^{3} \mathrm{He}$ is made, Eq. 10 proceeds more rapidly, providing most of the yield. The important point is that all of the reactions in Eqs. 9 and 10 proceed, so we indeed expect the neutron energy fraction to be roughly $26 \%$, as indicated by these equations (or perhaps slightly less when we consider the neutrons that interact before they leave the compressed fuel and the expellant).

In order to ignite DD (or any) fusion fuel, a driver must be used to hydrodynamically compress the capsule so the fuel temperature is raised to the temperature at which the fusion rate per gram is just larger than the electron bremsstrahlung loss rate per gram. Above this minimum fuel temperature, the ionizational energy losses of the charged fusion products can bootstrap the fuel to higher temperatures and produce what is called "runaway burn," which is what is meant by fusion burn. The minimum temperature for DT is about $4.5 \mathrm{keV}$, but the minimum for $\mathrm{DD}$ is $16 \mathrm{keV}$, and the minimum for $\mathrm{D}^{3} \mathrm{He}$ is $38 \mathrm{keV}$. Moreover, most target designs are based on achieving temperatures at least $50 \%$ larger than these minimums, to ensure reliable ignition. Unfortunately, it is somewhat impractical to compress a fuel to temperatures much above 10 to $20 \mathrm{keV}$ by normal means, so it is a challenge to ignite the advanced fuels by themselves, especially $\mathrm{D}^{3} \mathrm{He}$. (It is also more of a challenge to ignite these advanced fuels with the fast ignitor concept described in Sec. II.6 because the higher power requirement for the ignitor beam might make electrons of an energy too high to deposit energy locally enough to produce the required high ignition temperatures.) 
For these reasons, all advanced fuels considered here will be assumed to have a core of DT to act as a hotspot to ignite the advanced fuel. This core, if it is going to "trap" the charged reaction products, must be at least one alpha-particle mean free path in radius at the minimum burn temperature (i.e., 0.30 to $0.35 \mathrm{~g} / \mathrm{cm}^{2}$ ). To promote higher burn temperatures and hence more vigorous burn, we shall instead use $0.5 \mathrm{~g} / \mathrm{cm}^{2}$. In such a case, the fraction of the fuel mass that is tritium would be $0.2 \%$ of the total fuel mass if the total compressed fuel core column density is $\sim 3 \mathrm{~g} / \mathrm{cm}^{2}$, but only $0.04 \%$ if the total compressed fuel core column density is $\sim 5 \mathrm{~g} / \mathrm{cm}^{2}$. In either case, the hotspot burn temperature would be about $70 \mathrm{keV}$ (i.e., near the peak of the DT $<\sigma \mathrm{v}>$ curve). Such a hotspot causes the DD to burn typically at $\leq 100 \mathrm{keV}$.

In any fusion reaction, only a fraction $\mathrm{f}_{\text {burnup }}$ of the total fuel mass is able to burn before the target blows itself apart. This fraction, which is also called the burn efficiency, depends primarily on the column density $\mathrm{x}_{\text {fuel }}$ of the compressed fuel in $\mathrm{g} / \mathrm{cm}^{2}$, but also on a term $\Psi_{\mathrm{i}}$ dependent on the cross section at the burn temperature of the specific fuel utilized. A general expression, based on depletion of the needed ions, can be written as

$$
\mathrm{f}_{\text {burnup }}=\mathrm{x}_{\text {fuel }} /\left(\mathrm{x}_{\text {fuel }}+\Psi_{\mathrm{i}}\right)
$$

where $\Psi_{\mathrm{i}}$ is as follows:

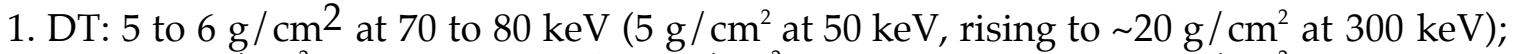

2. DD: $\sim 60 \mathrm{~g} / \mathrm{cm}^{2}$ at 70 to $80 \mathrm{keV}\left(\sim 70 \mathrm{~g} / \mathrm{cm}^{2}\right.$ at $50 \mathrm{keV}$, falling to $50 \mathrm{~g} / \mathrm{cm}^{2}$ at $100 \mathrm{keV}$, falling to $\sim 35 \mathrm{~g} / \mathrm{cm}^{2}$ at $\left.220 \mathrm{keV}\right)$;

3. $\mathrm{D}^{3} \mathrm{He}: \sim 50 \mathrm{~g} / \mathrm{cm}^{2}$ at $70 \mathrm{keV}\left(\sim 70 \mathrm{~g} / \mathrm{cm}^{2}\right.$ at $50 \mathrm{keV}$, falling to $\sim 39 \mathrm{~g} / \mathrm{cm}^{2}$ at $100 \mathrm{keV}$, falling to $\sim 34 \mathrm{~g} / \mathrm{cm}^{2}$ at $\left.220 \mathrm{keV}\right)$.

In short, $\Psi_{\mathrm{i}}$ is $\sim 5.5 \mathrm{~g} / \mathrm{cm}^{2}$ for DT, $\sim 60 \mathrm{~g} / \mathrm{cm}^{2}$ for DD, and $\sim 50 \mathrm{~g} / \mathrm{cm}^{2}$ for $\mathrm{D}^{3} \mathrm{He}$ (unless the advanced fuels can somehow be made to burn at temperatures well over $100 \mathrm{keV}$ ). Consequently, fburnup is roughly $35 \%, 4.8 \%$, and $5.7 \%$ for DT, DD, and $\mathrm{D}^{3} \mathrm{He}$ (respectively) for a capsule with a compressed column density of $3 \mathrm{~g} / \mathrm{cm}^{2}$, and $47.6 \%, 7.7 \%$, and $9.1 \%$ for a capsule with a compressed column density of $5 \mathrm{~g} / \mathrm{cm}^{2}$.

Target gain is equal to the product of the fuel mass, the burnup fraction $\mathrm{f}_{\text {burnup, }}$ various ablation and hydrodynamic efficiencies, the fusion energy release per gram, and $\left(1 / E_{d r}\right)$. Thus, for the same driver energy $E_{d r}$, the same fuel mass, and the same $x_{f u e l}$, the target gain for DD is only about one-seventh to one-sixth that for DT, due to the different burnup fractions, and the target gain for $\mathrm{D}^{3} \mathrm{He}$ is only about one-sixth to one-fifth that for DT. However, for an IFR with a magnetic thrust chamber, the important quantity is not the gain, but the product of the gain and $\mathrm{f}_{\text {debris, }}$, for it is the energy in charged debris plasma that is converted into thrust by the magnetic field. If this $\mathrm{Gf}_{\text {debris }}$ product is normalized to unity for DT, it is 0.42 to 0.51 for DD without expellant, and only one percentage point higher with $50 \mathrm{~g}$ of expellant.

Consequently, DD produces about half of the exhaust energy that DT produces, at the same mass flow rate, and hence a specific impulse only 70\% of that for DT. However, use of DD greatly reduces the tritium inventory, and also reduces the neutron emissions from $80 \%$ per reaction $(\sim 56 \%$ per target with expellant) down to $38 \%$ per reaction $(\sim 26 \%$ per target with expellant). We would therefore hope that there is the possibility of improving the DD target gain with creative ideas. 


\section{III.7.2 ${ }^{3}$ He Fuel}

The $\mathrm{D}^{3} \mathrm{He}$ form of fusion,

$$
\mathrm{D}+{ }^{3} \mathrm{He}=\alpha(3.6 \mathrm{MeV})+\mathrm{p}(14.7 \mathrm{MeV})
$$

releases $18.3 \mathrm{MeV}$ per interaction or $353 \mathrm{MJ}$ per mg of $\mathrm{D}^{3} \mathrm{He}$ (assuming 100\% burn up). Again, the total energy release per binary collision is about the same as for DT. Note, however, that $100 \%$ of the energy appears as kinetic energy of charged particles at each reaction site-that is, the fuel is "aneutronic" at production. All of the emitted particles in Eq. (13) deposit their energies inside a compressed fuel capsule having 3 to $5 \mathrm{~g} / \mathrm{cm}^{2}$, so we might expect this fuel to emit no neutrons and hence nothing but $x$ rays and chargedparticle plasma debris.

In actuality, however, some DD fusion will occur, even though the $\mathrm{D}^{3} \mathrm{He}$ cross section is somewhat larger than the DD cross section, so $\mathrm{D}^{3} \mathrm{He}$ is not aneutronic as applied to ICF. The situation is even worse when the $\mathrm{D}^{3} \mathrm{He}$ is ignited with a DT hotspot, because the 14.1$\mathrm{MeV}$ neutrons from the DT hotspot convert some of the ${ }^{3} \mathrm{He}$ and make tritium that can burn with the deuterium $\left(\mathrm{n}+{ }^{3} \mathrm{He}--->\mathrm{p}+\mathrm{T}\right) .{ }^{40}$ Much of the yield then comes from the DT reactions. Even at large target sizes that achieve high burn temperatures, 10 to $40 \%$ of the plasma emission is in the form of $\mathrm{x}$ rays having more than $100 \mathrm{keV}$ and hence ranges similar to neutrons. ${ }^{41}$ The net result here is that perhaps $\sim 40 \%$ of the energy of each reaction ends up as neutron kinetic energy. When Eq. (6) is used to estimate the neutron energy leaving the target (and expellant), we estimate that $28 \%$ of the energy emerges in the form of neutrons. Thus, $\mathrm{D}^{3} \mathrm{He}$ is definitely not aneutronic! The rest of the energy is typically split between $x$ rays and the debris plasma, according to Eqs. (6) and (7), so about $36 \%$ of the total energy is left to emerge as charged debris plasma kinetic energy, just as for DD fuel.

In the previous section, we showed that the burnup fraction for $\mathrm{D}^{3} \mathrm{He}$ is 5.7 to $9.1 \%$ respectively for compressed target column densities of 3 to $5 \mathrm{~g} / \mathrm{cm}^{2}$. The $\mathrm{Gf}_{\text {debris }}$ product (i.e., the fractional energy into charged debris plasma kinetic energy) for $\mathrm{D}^{3} \mathrm{He}$ is hence only 40 to $57 \%$ of that for DT, so the specific impulse at the same mass flow rate should be only $~ 70 \%$ of that for DT, just as for DD.

For VISTA, we therefore see that $\mathrm{D}^{3} \mathrm{He}$ is not as good for an IFR as one might at first have thought. In fact, it appears to be about the same as DD in all performance aspects. In addition, $\mathrm{D}^{3} \mathrm{He}$ has two other distinct disadvantages for IFR applications: (1) there is no major source of ${ }^{3} \mathrm{He}$, so its use might necessitate mining the lunar surface and/or the Jovian atmosphere to obtain sufficient ${ }^{3} \mathrm{He}$, and perhaps even worse, $(2)^{3} \mathrm{He}$ has no convenient cryogenic phase that is commensurate with deuterium (deuterium freezes before ${ }^{3} \mathrm{He}$ liquefies, so the two substances tend to separate). ${ }^{42}$

\section{III.7.3 General Comments about Advanced Fuels and Antimatter}

The simplistic calculations presented in the previous two sections suggest that the advanced fuels will not outperform DT, unless the burn temperature for these fuels can be significantly increased. Furthermore, we have not discussed the possibility of seeding these fuels with tritium, which greatly increases their yields. We leave to further studies just how one might optimize the performance with advanced fuels. 
Antimatter fuels are not considered to be a serious option for VISTA for many reasons. As we demonstrate in Section $\mathrm{XV}$, the performance with antimatter is very discouraging because the antimatter-matter annihilation produces pions and gamma rays that do not readily interact with matter because of their long mean free paths in both the fuel and the expellant. Consequently, antimatter-matter annihilations produce little charged plasma debris that is usable in VISTA's magnetic thrust chamber, and the charged-pion products themselves have energies that are much too large to be efficiently redirected by a magnetic field of any practical strength. Antimatter is also very difficult and costly to produce and store, let alone transport, although some advances are being made. Still, the cost of producing antimatter is enormous in comparison to the rather expensive cost of tritium, and without further innovation, similar masses of tritium and antimatter would be required for VISTA (see Sec. XV). Thus, were it not for the discouraging performance with antimatter, its cost would still be prohibitive enough to discourage its use.

In passing, it is important to note that a fusion target containing $\mathrm{DD}$ or $\mathrm{D}^{3} \mathrm{He}$ produces a single release of charged particles in the form of an expanding cloud of debris plasma consisting of heated capsule, hohlraum, and expellant material-there are few high-energy $(\mathrm{MeV})$ charged particles streaming away from the fusion site. The same is true for DT fuel. Therefore, when the debris from the advanced fuels is used with the VISTA magnetic thrust chamber, the jet efficiency is not reduced as it would be if the fuels produced a bimodal (e.g., double-peaked) energy distribution of released charged particles (see Secs. V.7 and XVI.1).

\section{III.8 Target and Propellant Storage}

Roughly 4,100 metric tons of expellant must be stored onboard for a VISTA mission to Mars. If it is hydrogen, the expellant could be stored as a liquid in tanks formed as a frustum extension of the open end of the spacecraft. If the radius of the cone at the open end is about $200 \mathrm{~m}$ and the thickness of the cone at this location is $9 \mathrm{~m}$ (both to allow for crew quarters and because the coil shield has a shadow at least this large), then 4,100 tons of hydrogen at density $0.077 \mathrm{~g} / \mathrm{cm}^{3}$ can be stored in $5.3 \times 10^{4} \mathrm{~m}^{3}$. This volume requires a tank whose circular cross section has a radius of only $3.7 \mathrm{~m}$, so the propellant tanks will easily fit inside a $9 \mathrm{~m}$ extension of the cone.

These expellant tanks would require at least 10\% tankage structure for containment and support, and would hence be at least 410 tons. Such tanks would intercept some scattered neutrons, and more importantly, would view some spacecraft radiator surfaces operating at $900 \mathrm{~K}$. Although these tanks would be designed to minimize this heat input by keeping them in the conical surface and/or using appropriate shields, the tanks or their shields will still see a portion of the spacecraft (radiator) interior. We leave to a future study just how much heat these tanks might absorb. Nevertheless, we emphasize that this is an important issue because any absorbed heat would be removed with a cryogenic refrigerator operating at perhaps 200 absorbed-watts per refrigerated-watt and $20 \mathrm{~kg} / \mathrm{W}$ specific refrigerator mass. The mass of such refrigeration systems must not be large.

About 3.72 metric tons of DT fuel must be stored onboard for a Mars mission. The deuterium fuel could be stored in the same way as the hydrogen expellant, and represents a very minor perturbation of the hydrogen mass and tankage. On the other hand, the 2.23 tons of tritium must be stored as a gas due to its radioactive decay heat of $0.324 \mathrm{~W} / \mathrm{g}$. If 
the tritium mass $\mathrm{m}_{\mathrm{T}}$ (tons) is stored in a spherical tank of radius $\mathrm{r}_{\mathrm{tank}}$ meters, its equilibrium temperature $T_{\text {tank }}(\mathrm{K})$ would be

$$
\mathrm{T}_{\text {tank }}=820\left[\mathrm{~m}_{\mathrm{T}} / \mathrm{r}_{\operatorname{tank}}\right]^{1 / 4} .
$$

For material of strength stank (psi) at this temperature and a strength safety factor of $\mathrm{f}_{\text {safety, }}$ and density $\rho_{\operatorname{tank}}\left(\mathrm{g} / \mathrm{cm}^{3}\right)$, the thickness of the tank $\mathrm{t}_{\text {tank }}(\mathrm{m})$ would have to be at least

$$
t_{\text {tank }}=2 \times 10^{4} \mathrm{~m}_{\mathrm{T}}^{5 / 4} \mathrm{r}_{\text {tank }}-5 / 2\left(\mathrm{f}_{\text {safety }} / \mathrm{s}_{\text {tank }}\right)
$$

and the tank mass $m_{\text {tank }}$ (tons) would be

$$
\mathrm{m}_{\text {tank }}=2.5 \times 10^{5}\left(\rho_{\text {tank }} \mathrm{f}_{\text {safety }} / \mathrm{s}_{\text {tank }}\right) \mathrm{m}_{\mathrm{T}}^{5 / 4} \mathrm{r}_{\text {tank }}{ }^{-1 / 2} \text {. }
$$

For example, SS304 stainless steel (density $7.8 \mathrm{~g} / \mathrm{cm}^{3}$ ) has a strength of about 28,000 psi near $400 \mathrm{~K}$, so storage of 2.3 tons of tritium with $\mathrm{f}_{\text {safety }}=4$ and $\mathrm{r}_{\text {tank }}=6.3 \mathrm{~m}$ would require a 309-ton tank of thickness $7.9 \mathrm{~cm}$ operating at $400 \mathrm{~K}$. A tank of this size will just fit inside the projected $13 \mathrm{~m}$-thick shadow of the coil shield at this location, but is obviously very massive. Clearly, future studies should seek a more favorable design, which should be able to reduce the mass by at least a factor of five with special materials and a slightly reduced safety factor; we assume that such an advanced design can be developed for VISTA. In any case, a desire to avoid excessive tankage mass for tritium-loaded fuels is a good reason to minimize the use of DT fuel, and to make tritium en route (see next Sec.).

Storage of pre-fabricated unfilled targets with their expellant foam substrates would require slightly more volume than the cryogenic expellant $\left(5.3 \times 10^{4} \mathrm{~m}^{3}\right)$, and may therefore be impractical. Storage of pre-fabricated unfilled targets excluding their expellant foam substrates would require only a few percent tankage. The target storage mass would then be a minor perturbation on the storage mass for the expellant and the tritium.

\section{III.9 Onboard Production of Tritium}

It is disadvantageous for VISTA to transport a lot of tritium for a number of reasons: (1) there is potential hazard transporting large quantities of tritium up through the atmosphere (see Sec. X.1.1); (2) a radioactive material represents a hazard for onboard personnel; and (3) tritium is very costly. The cost of small quantities of tritium can be estimated to be about $\$ 22 \mathrm{k} / \mathrm{g} .{ }^{43}$ For a mature process in a fusion economy, we must still expect the price to be at least $\$ 2 \mathrm{k} / \mathrm{g}$ to $\$ 3 \mathrm{k} / \mathrm{g}$. This price means that the tritium for a fast Mars transport would cost at least 5 to 7 billion dollars. In addition, it is very advisable to try to generate tritium on board for trips to the outer planets and beyond because the tritium half life is 12.3 years.

We therefore recommend studying ways to reduce tritium requirements at launch time from the maximum of 2.23 metric tons $(2,230 \mathrm{~kg})$ for a Mars roundtrip using DT fuel to something closer to several $\mathrm{kg}$. This might be accomplished by using DD fuel with a DT hotspot, which requires only $2.2 \mathrm{~kg}$ of tritium, that is, $4.4 \times 10^{26}$ tritons (see Sec. XV.1). Because rather hot $(1500 \mathrm{C}$ ) lithium is used as the superconducting magnet shield coolant,

tritium can be bred from the total number of neutrons emitted from the target site $\left(10^{29}\right.$ per 
Mars roundtrip) and extracted by cold trapping. Of the total number of neutrons emitted, $3.93 \%$ (i.e., $\sim 4 \times 10^{27}$ ) intercept the shield and can undergo multiple tritium breeding reactions (1.8 T are produced per $14.1 \mathrm{MeV}$ neutron in an infinite medium). Thus, because the $\sim 80-\mathrm{cm}$ lithium breeder zone would be rather neutronically thick $(15 \mathrm{~cm}$ being the mean free path for $14 \mathrm{MeV}$ neutrons), the favored reaction would be the high energy one ${ }^{7} \mathrm{Li}\left(\mathrm{n}_{\text {fast }} \mathrm{n}^{\prime} \mathrm{He}\right) \mathrm{T}$ rather than the thermal and epithermal reaction of ${ }^{6} \mathrm{Li}\left(\mathrm{n}_{\text {slow }}, \mathrm{He}\right) \mathrm{T} \cdot{ }^{44}$ If the production and recovery fraction could be only as high as half of the infinite-medium rate, all of the tritium could be bred en route (except for a start-up amount). Detailed neutron transport calculations will be needed to optimize the system and see how much tritium can be practically produced. The outcome of such a study could affect the target design by determining how much tritium is available for each target. In any case, it is obvious that a major fraction of the onboard tritium can be bred en route and not taken up through the atmosphere if DD fuel is used. Such breeding would necessitate bringing along additional (negligible) quantities of lithium, but would require the extra mass required for the tritium recovery system.

We note that the shield radiators have been sized assuming that all of the neutron energy that strikes the shield is absorbed. Reaction ${ }^{7} \mathrm{Li}\left(\mathrm{n}_{\text {fast }} \mathrm{n}^{\prime} \mathrm{He}\right) \mathrm{T}$ absorbs $2.47 \mathrm{MeV}$, so tritium breeding would lessen the burden on the radiators. We also note that lithium is easy to circulate and has received consideration for space applications. Future studies will have to emphasize low-mass, easy-to-maintain equipment to do the tritium handling and target fabrication en route. Additional comments are made in Sec. XIV.4.

Burning D- ${ }^{3} \mathrm{He}$ is also a possibility but requires getting large inventories of ${ }^{3} \mathrm{He}$, which are hard to obtain. For this reason, and difficulties in cryogenic fabrication of targets, as well as the lack of any significant advantage of $\mathrm{D}^{3} \mathrm{He}$ over DD (see Sec. XV), there may be no reason to pursue $\mathrm{D}^{3} \mathrm{He}$ unless creativity can overcome its practical limitations. 


\section{DRIVER SYSTEMS}

The system that supplies laser power to compress and ignite the target is called the driver. Because the driver operates repetitively at tens of Hertz, it requires a pulsed power conditioning system. The driver also needs its own radiator system to reject waste heat because it is rather inefficient in converting electrical energy into laser energy. The driver itself is composed of many modules, both for redundancy and to enable one or two final beams to be assembled from smaller-diameter beams without exceeding the maximum allowed fluence on any optical component (e.g., lenses, mirrors). Figure I-1 shows two final turning mirror assemblies directing the final beams toward the target, with the beams arranged into multiple beam cones. Table IV-1 shows the features desired for VISTA's driver, as compared with current capability.

Table IV-1 Desired Features of VISTA's Target Driver

\begin{tabular}{|l|c|c|}
\hline \multicolumn{1}{|c|}{ Item } & Desired Value & Current Capability \\
\hline \hline Type of target driver & DPSSL $^{*}$ & Prototypes already exist \\
\hline Driver efficiency & $6 \%$ & $3-10 \%$ \\
\hline Waste heat rejection T & $900 \mathrm{~K}$ & $\sim 300 \mathrm{~K}$ \\
\hline Driver energy & $5 \mathrm{MJ}$ & $\begin{array}{c}\sim 2 \mathrm{~J} \\
\text { (100 J under construction) }\end{array}$ \\
\hline Rep rate & $1-30 \mathrm{~Hz}$ & $1-25 \mathrm{~Hz}$ \\
\hline Shape of driver pulse & Pulse-shaped (not square) & Gaussian or square \\
\hline Reliability & $10^{8}$ pulses & $10^{9}$ for Nd:YAG lasers \\
\hline Mass & $\sim 150$ tons (w/o radiators) & $\begin{array}{c}\sim 10^{4} \text { tons at } 2 \mathrm{MJ} \\
\text { (Nd:glass laser) }\end{array}$ \\
\hline
\end{tabular}

${ }^{*}$ Diode-Pumped Solid-State Laser

\section{IV.1 A Survey of Terrestrial Driver Developments}

In order to choose the best concept for an IFR driver, we first examined the attributes and disadvantages of the drivers being developed for terrestrial ICF applications: heavy-ion beams, light-ion beams, free-electron lasers (FELs), krypton-fluoride (KrF) lasers, compact toruses (CTs), and diode-pumped solid-state lasers (DPSSLs).

A heavy-ion accelerator is one candidate for a terrestrial driver due to its high efficiency $(\leq 35 \%)$ and its use of established accelerator technology. A typical driver of this type could accelerate singly charged uranium or lead ions to a total energy of about $10 \mathrm{GeV}$ (i.e., $10^{10} \mathrm{eV}$ ). These ions would then be focused on the target. However, because this type of driver requires an accelerator, it is very massive. A typical example would include an induction accelerator section $3 \mathrm{~km}$ long at about 7 tons / meter (due to the iron magnets) plus transport sections $1 \mathrm{~km}$ long at about 0.1 tons $/$ meter. ${ }^{45}$ Obviously, such a device is too long and prohibitively massive for use on an IFR, unless it could be completely redesigned in concept and length so that its total mass were less than about 500 tons.

The light-ion beam concept is based on the limited development of the past two decades at Sandia National Laboratory to accelerate lithium ions to about $30 \mathrm{MeV}$ using 
pulsed-diode technology. Ions would either be focused on the target, or the diode energy could be discharged directly at the target through a mesh of small-diameter wires so as to create a plasma to implode a target. The direct application of the diode current through a set of wires would be extremely challenging in space. A great deal more development is necessary to prove the effectiveness of either of these applications as a terrestrial driver, even though the current machines (PBFA-II, Z) may ultimately attain an efficiency of 7 to $14 \%$. Critical design questions remain pertaining to the demonstration of the capability of producing the required power on target, the survivability of a diode for $10^{8}$ IFR pulses (the diode is destroyed every pulse in PBFA-II), the pulse-shaping capability, and the transport characteristics of the beam from the diode to the target. The greatest concern for IFR applications is that the light-ion concept involves a relatively short focusing distance, and thus inherently large beam divergence. Such a beam can not propagate without the aid of a plasma channel to guide it, and such a channel may require a gas pressure on the order of 10 Torr. Thus, the current light-ion-beam concept will not propagate through a vacuum (i.e., in space), and hence cannot be used in an IFR. It may be possible, however, to lengthen the diode to reduce the beam divergence, and to add co-moving cold electrons to neutralize the beam. This would permit propagation in a vacuum, but the development of such a scheme may be impractical.

FELs have also not been developed much beyond the concept-demonstration stage, so much development work is required. Such a driver employs an electron accelerator, plus a wiggler transport section in which the electron energy is transferred to laser light. The laser light is then focused on the target. One terrestrial advantage of an FEL is its maximum efficiency, estimated to be between $15 \%$ and $20 \%$. Another advantage is that it produces light at $1 / 4 \mu \mathrm{m}$ wavelength, which is ideal for target implosions. However, FEL systems are very massive: each terrestrial FEL beam may involve 200 tons for the accelerator plus 50 to 100 tons for each of at least 10 wiggler sections (i.e., about 800 tons per beam). ${ }^{46}$ Critical design issues include the attainment of a beam brightness at least 100 times greater than the maximum obtained to date, and the capability for pulse-shaping. In addition, the driver temperature can not exceed about $400 \mathrm{~K}$, due to the use of ferromagnetics. An overriding constraint, however, is that its diffraction-limited beam is not steerable in distances of interest (less than a kilometer), because the beam is too intense to allow the use of mirrors. Although one might expect that the beam could be expanded and then steered, no viable technique to accomplish this has yet been developed even in theory. Thus, the FEL is not applicable to an IFR unless its mass can be reduced and some technique for steering the beam can be developed.

The $\mathrm{KrF}$ laser driver generates a beam already at $1 / 4 \mu \mathrm{m}$ wavelength, and is capable of high repetition rate operation (because its lasing medium is a gas). The typical efficiency of such a driver is estimated to be between 3 and $6 \%$ up to about $600 \mathrm{~K}$, then dropping to about half this efficiency by $1000 \mathrm{~K}$ (due to thermal de-excitation); however, it is possible that all of these efficiency values might double with future technological developments. ${ }^{47}$ The typical $\mathrm{KrF}$ driver operates by accelerating an electron beam, passing the electron beam through a foil that separates the acceleration region from the $\mathrm{KrF}$ gas, and using the electrons to excite the $\mathrm{KrF}$ laser state. Critical design developments must address the lifetime of the foil, the beam quality degradation from density inhomogeneities in the gas, the long-term damage for $1 / 4 \mu \mathrm{m}$ light on mirrors and other optical elements, and the lack of sufficient beam brightness. In short, a $\mathrm{KrF}$ driver could be appropriate for an IFR only with considerable development, if then. 
The compact torus (CT) accelerator technology is a possibility, but is not being developed at this time. In this concept, a doughnut-shaped magnetized cloud of ions is accelerated, focused, and converted to $x$ rays, which are directed towards the target. Critical design problems must address the capability for any pulse shaping, and the possibility of hot-electron preheat on the target generated by the large magnetic field time derivative upon interaction of the torus with the target. ${ }^{48}$ More importantly, the material that guides the CT to the target is destroyed each shot. Such a situation may be acceptable for terrestrial application because the material can be recycled, but for space application, this material would cause significant degradation of jet efficiency.

The DPSSL is the most developed technology, which is based on gain media that are doped crystals such as $\mathrm{Yb}^{3+}$-doped fluorapatite $\mathrm{Sr}_{5}\left(\mathrm{PO}_{4}\right)_{3} \mathrm{~F}$ [i.e., $\mathrm{Yb}$ :S-FAP] instead of the neodymium-doped glass typical of solid-state lasers of the past. These lasers are solid-state lasers, so they have a very mature experimental base consisting of decades of experimentation on terrestrial Nd:glass lasers. A DPSSL driver with the above gain medium can generate laser light at $1.047 \mu \mathrm{m}$ wavelength, which can be frequency-shifted to $1 / 3$ or $1 / 2 \mu \mathrm{m}$ for efficient coupling to the target, and then focused on the target. Methods of cooling the crystals for high rep-rate operation have already been developed, enabling DPSSLs to achieve 25-Hz operation, but at only $2 \mathrm{~J}$ of output. A 100-J Yb:S-FAP DPSSL (the Mercury Laser) is now under construction at LLNL that expects to achieve efficiencies of about $10 \%$, but it is merely one example of many DPSSLs worldwide. Important design issues include a determination of the maximum operating temperature, and the degree of beam quality (smoothness) required on target. Based on current information, DPSSLs are clearly the best candidate for VISTA, especially if ceramic gain media can be developed.

\section{IV.2 What is a Laser?}

In the next Section, we will explain why a DPSSL is currently the best choice for VISTA's driver. It may therefore be helpful at this point to understand what a laser really is.

When certain materials are subjected to an intense burst of light from something like a xenon flashlamp, which is similar to the light used to time automobile engines (but more intense), the atoms in the material are raised to an "excited" state. Rather than de-excite very quickly (in nanoseconds) down to the original "ground" state, as most materials do, the atoms in these special materials instead de-excite only slightly, down to a "metastable" state that tends to stay excited for a considerable length of time (hundreds of microseconds to about one millisecond). If a light beam traverses these materials while the atoms are in this metastable state, and if all the light rays (photons) in the beam have an energy exactly equal to the energy difference between this metastable state and the ground state, then the light beam will stimulate these excited atoms to de-excite by creating an additional photon having the same energy as the beam photons and traveling in the same direction. Thus, the original light beam has been amplified. The term "laser" merely describes this process as Light Amplification by Stimulated Emission of Radiation (LASER). Thus, the term laser is actually an acronym.

The special materials having such lasing properties are called laser materials or gain media, because they produce gain. The materials are usually made by adding a special dopant substance (e.g., neodymium ions) to a host material like glass, and it is the dopant ions that have the metastable states. The laser is then named by these two ingredients (e.g., $\mathrm{Nd}$ :glass, meaning neodymium-doped glass). Such laser materials are said to be "pumped" 
by the light from the flashlamps because some of the flashlamp energy becomes "stored" in the lasing material for a certain "storage time." Thus, such a laser might be referenced as a flashlamp-pumped Nd:glass laser. The original light beam is called the "injected" beam, and the process of removing the energy from the excited states is called "extraction." People using lasers must then be concerned about imperfections in the optics that transport the amplified light beams from place to place, because such imperfections can re-orient the light rays so that they are not all traveling in exactly the same direction. Such disruption of the light during propagation is referred to as "wavefront distortion," which causes the light to be less able to focus to what is desired when lasers are used to illuminate an ICF target-which is a very small intense spot. To eliminate some of these distortions, some laser applications require the beams to be "smoothed."

\section{IV.3 Choice of a DPSSL for VISTA's Driver}

It is clear from Section IV.1 that a DPSSL is the most likely candidate for VISTA's driver, both from the perspective of the maturity of solid-state lasers (SSLs) and the theoretical expectation that DPSSLs can indeed be adapted for space applications. Of course, DPSSLs are not fully developed, but they have already demonstrated amazing progress: 1) a 2-J DPSSL has demonstrated operation at $25 \mathrm{~Hz}, 2$ ) SSLs have demonstrated the illumination and subsequent implosion of real ICF targets, and 3) the NIF, which is a singleshot machine, is expected to demonstrate capsule gains of 10 to 20 in the first decade of the $21^{\text {st }}$ century using only $1.8 \mathrm{MJ}$. The engineering development of a terrestrial power plant based on a DPSSL driver could follow this rather quickly. As a matter of fact, perhaps the most complete systems analysis ever conducted for a terrestrial fusion power-plant driver has already been completed, and it was for a 3.6-MJ DPSSL. ${ }^{49}$

It is important to note that the DPSSL approach is directly related to flashlamppumped Nd:glass solid-state lasers with regard to pumping, energy storage, extraction, multi-pass amplifier architecture, light propagation, linear and nonlinear wavefront distortions, frequency conversion, temporal pulse shaping, and beam smoothing. The DPSSL approach therefore builds on the extensive solid-state laser and target development accomplished during the last two decades with Nd:glass lasers. In particular, DPSSLs represent an extension of SSL technology toward inertial fusion energy (IFE) - that is, terrestrial power production-AND space applications because they can employ the following:

- Narrow-frequency-band laser-diode arrays instead of wide-frequency-band flashlamps to pump the gain media, therefore offering higher pump efficiency with less heat deposition because more of the pump light is absorbed by the laser gain medium.

- Yb:crystal ${ }^{50}$ or doped-ceramic gain media instead of Nd:glass, therefore offering four times the thermal conductivity, thus permitting near-sonic helium cooling ${ }^{51}$ of the gain media for rep-rated operation without stress fracture of the gain medium.

The Yb:crystal gain media also provide an energy storage lifetime of $1.1 \mathrm{~ms}$, which is three times larger than that for Nd:glass; the longer storage lifetime reduces the cost required for the laser diodes. Therefore, if the price of diodes decreases in response to a growing market size, a DPSSL driver may turn out to be practical because a DPSSL appears as though it might be able to (1) scale to MJ output energies on target and (2) achieve an affordable development path because of its modularity. To this end, a number of DPSSL facilities have been envisioned at LLNL to integrate the approaches and develop new capabilities for 
irradiating inertial-fusion targets. These begin with the Mercury Laser having $100 \mathrm{~J}$ at 10 $\mathrm{Hz}^{52}$ then an Integrated Research Experiment (IRE) laser driver delivering a few $\mathrm{kJ}$, leading eventually to a MJ-class driver. This DPSSL development track could proceed in parallel with NIF target research.

It is also important to note that a 5-MJ DPSSL for VISTA would be composed of perhaps hundreds of beams, each beam being a collection of smaller beamlets, and each beamlet being essentially a DPSSL by itself-just as envisioned for a terrestrial power-plant driver. ${ }^{53}$ The reason for this subdivision is to be able to grow the crystalline gain media only at the size required for the beamlets, whereas the beams could use the larger-sized fused-silica $\left(\mathrm{SiO}_{2}\right)$ lenses and mirrors that have typically been used at ICF research facilities to date. Such modularity of a DPSSL means that its functionality can be validated at the component level or at the single-beamlet level, thus permitting experimental verification at reduced development cost. In addition, many of the novel laser-diode technologies envisioned for a terrestrial IFE driver are relevant to a variety of scientific, industrial, and military applications and will therefore continue to advance in parallel with ICF developmental efforts.

One area of particular importance for a direct-drive DPSSL is the achievement of adequate beam smoothness on target. We mention this subject here, not because it is relevant for VISTA's indirect-drive targets, but because it was originally thought to be an issue, perhaps even for indirect drive; however, it is now known that beam smoothness is only a minor issue for VISTA's DPSSL. Specifically, sufficient laser bandwidth $(\geq 1 \mathrm{THz})$ is required for direct-drive targets to avoid implanting perturbations during the early stages of a capsule's implosion that can lead to significant hydrodynamic instability growth. Calculations now show that a DPSSL can achieve this required beam smoothness. ${ }^{54}$ In particular, DPSSLs have the modularity to permit separate generation of the initial "foot" and following "main" portions of a capsule temporal drive pulse. These portions of the temporal pulse can be subsequently combined using a total solid angle at the final turning mirror(s) of order 5\% or less. The foot pulse could comprise 10 to $15 \%$ of the energy using $\mathrm{Nd}$ :glass amplifiers and multi-crystal triplers to produce as much as $3 \mathrm{THz}$ of bandwidth. Even though the tripler might be operated at reduced efficiency, the consequence to the overall efficiency would be mitigated because the foot pulse contains only a small fraction of the total pulse energy. Such a large bandwidth laser configuration for the foot pulse would minimize seeding of Rayleigh-Taylor instabilities in the initial implosion. The main pulse could be an assemblage of beamlets operating at slightly different wavelengths, using the four gain peaks (i.e., four colors) available from the ytterbium-doped fluorapatite gain media [i.e., based on $\mathrm{M}=\mathrm{Sr}, \mathrm{Ca}$, and $\mathrm{Ba}$ in the chemical formula $\left.\mathrm{Yb}^{3+}: \mathrm{M}_{5}\left(\mathrm{PO}_{4}\right)_{3} \mathrm{~F}\right]$. Each of these gain peaks is known to have $\sim 0.3 \mathrm{THz}$ bandwidth, so the assemblage of beamlets can have sufficient beam smoothing for the main peak (and $\sim 80 \%$ conversion to UV). In addition, relative to the foot pulse, the main-pulse beams could offer a smaller spot size to accommodate the reduced size of the imploding target. Such a multi-color approach provides a net beam smoothness that exceeds what the NIF will be able to achieve for a bandwidth of $1 \mathrm{THz}$.

The development of a DPSSL is proceeding at many laboratories in the U.S., Japan, and elsewhere. Although the Mercury laser now under construction at LLNL is merely one example, we describe it here for illustrative purposes. The goals of the Mercury Project ${ }^{55}$ are to produce a DPSSL delivering $100 \mathrm{~J}$ with an overall electrical efficiency of $\geq 10 \%$ at the first harmonic $(1047 \mathrm{~nm})$, a final focus less than 5 times the size for a diffraction-limited beam, pulse lengths of 2 to $10 \mathrm{~ns}$, and a repetition rate of $10 \mathrm{~Hz}$. Although small in scale, the Mercury laser will demonstrate the utility of a DPSSL as an IFE power plant driver in terms of pulse duration, laser efficiency, and thermal management, but not in total energy output 
(although the energy output will be more than an order of magnitude larger than any other pulsed diode-pumped laser ever built). Subsequent research must demonstrate efficient average-power frequency conversion and beam smoothing, and temporal pulse shaping that permits an overall efficiency of $10 \%$.

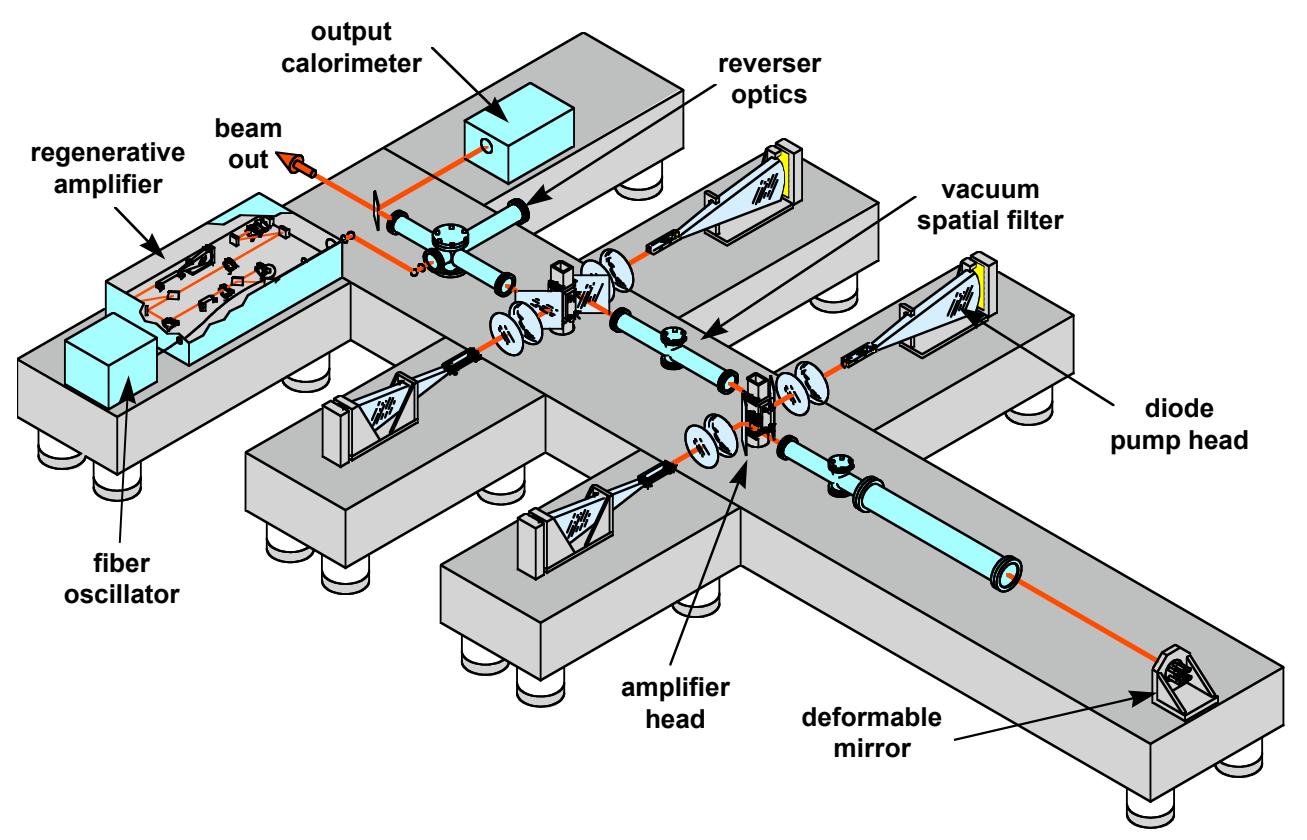

Fig. IV-1 Table layout of the Mercury baseline architecture.

The original baseline architecture for Mercury (Fig. IV-1) consists of a four-pass cavity with angular multiplexing and a reverser section to reverse the beam direction between passes two and three. Each of the two amplifier heads contains seven Yb:S-FAP crystals each $0.75 \mathrm{~cm}$ thick and at the appropriate size for the $3 \times 5 \mathrm{~cm}^{2}$ active beam area. ${ }^{56}$ Each amplifier head is pumped along the laser beam direction by two arrays of $900-\mathrm{nm}$ laser diodes, one array with its homogenizer tube and telescope optics on each side of the amplifier head. During a 1-ms pump pulse, each laser diode array exhibits thermal sag $<10 \%$ and acceptable thermal chirp (linewidth $<5 \mathrm{~nm}$ FWHM) ${ }^{57}$ VISTA must use a design much better than that for Mercury, however, for two reasons: (1) the reverser architecture must be replaced by the more robust NIF-type Pockels Cell optical switch architecture (which Mercury couldn't use because a high-power Pockels Cell wasn't available); and (2) the gain medium must have at least 2 to 3 times the transverse dimension that is currently possible for growth of $\mathrm{Yb}$ :S-FAP crystals. The latter therefore requires further crystal development, or (e.g.) development of isotropic doped-ceramic gain media (which would be highly preferred).

A laser driver must be affordable and environmentally acceptable as well as technically viable. The direct affordability of a DPSSL driver depends primarily on the price of the laser diodes, and DPSSL drivers will be practical only if the expected price reductions occur as volume production in a fusion economy begins. In addition, the final optics must be protected from x-ray and neutron bombardment to enable the long life required for minimal maintenance. Low-dose experimental exposures of fused-silica materials support the idea that final optics for a terrestrial DPSSL driver can be operated at $400{ }^{\circ} \mathrm{C}$ to continuously anneal defects from neutron interactions for final wavelengths $\lambda \geq \sim 350 \mathrm{~nm}$, but high-dose experimental verification is lacking. 
In summary, DPSSLs exist, are rep-ratable, satisfy most of the requirements for an IFR driver, and appear to have a relatively straight-forward affordable development path for terrestrial applications. The further development required for space applications might seem to be nominal, but the development of terrestrial DPSSL and SSL systems has not addressed either the total system mass (which is perhaps the most important feature for an IFR driver) nor VISTA's driver operating temperature requirement of $900 \mathrm{~K}$. Application of a DPSSL to VISTA may therefore require development beyond the extensive development already planned for terrestrial DPSSL applications in the energy, scientific, military, and commercial fields. In addition, the lifetimes and damage thresholds for DPSSL laser optics and coatings operating at sub-micron wavelengths, which are currently not well known (especially in the presence of high neutron fluences), represent extrapolations beyond current technology.

Concerning the operation of a DPSSL at temperatures higher than the terrestrial operating point of $\sim 330 \mathrm{~K}$, we have found by running our DPSSLIFE systems analysis code for terrestrial IFE drivers that the efficiency of such a DPSSL can decrease by over a factor of three for operation above $800 \mathrm{~K}$ because of reduced extraction efficiency for Yb:S-FAP crystals. To be sure, this performance is much superior to that of a Nd:glass SSL, which suffers significantly in efficiency for temperatures above $\sim 420 \mathrm{~K}$ because the lower laser state becomes thermally populated. Nevertheless, the terrestrial system is not designed with space applications in mind, nor for maximum efficiency. Thus, we leave to a future study the determination of the maximum effective operating temperature of a (possibly doped-ceramic) DPSSL, and in particular, whether such a DPSSL can achieve an efficiency of at least 6\% operating at a temperature of at least $600 \mathrm{~K}$ (see Sec. XI.1). We also leave to future study the determination of the minimum mass of a DPSSL driver mounted on the VISTA radiator trusses. The crystals and optics will be several tens of tons, but the amount of stabilizing structure for the optics is a key but rather uncertain mass component. For parametric studies, we assumed a minimum mass including power conditioning subsystems of $50+20 \mathrm{E}_{\mathrm{dr}}$ tons, with the driver output energy $\mathrm{E}_{\mathrm{dr}}$ in $\mathrm{MJ}$.

\section{IV.4 A Conceptual Driver Alternative}

Although it seems as though a DPSSL is a good candidate for VISTA's driver, it may not prove adaptable for the reasons stated in the previous Section. If DPSSLs are unable to work for VISTA, we would then need a backup. Based on the information presented in Section IV.1 and the anticipated developments in gaseous lasers, we believe that the best backup driver system for VISTA would be a gaseous excimer laser operating with $6 \%$ efficiency at a waste-heat-rejection temperature of $900 \mathrm{~K}$ (as required in Table IV-2). This system is like a $\mathrm{KrF}$ system, which seems undesirable now, but we left the gas medium unspecified pending future developments that should overcome the few disadvantages of the $\mathrm{KrF}$ system. We chose these efficiency and temperature specifications independently, but they are identical to those used by $\mathrm{Hyde}^{58}$ for $\mathrm{KrF}$.

The primary way that the driver system affects spacecraft performance is through the total mass of the driver equipment, the power-conditioning equipment, and the radiators for both subsystems. System studies indicate that the most important feature of the baseline driver is the mass of the laser driver subsystem itself-not the masses of the power subsystem or radiators. In fact, our studies indicate that IFR performance is near optimum for any efficiency near or above 5\%, and any operating temperature above about $500 \mathrm{~K}$, provided the driver with its power-conditioning subsystem (but not its radiators) can maintain a mass $\leq 300$ tons for $5 \mathrm{MJ}$ on target. That is, in the baseline VISTA design, the total 
driver system represents about $4.5 \%$ of the total spacecraft launch mass, and the waste-heat radiators represent about $44 \%$ of the total driver mass. If the radiator temperature drops much below $500 \mathrm{~K}$, or the driver efficiency drops below $5 \%$, the radiator mass begins to significantly increase the total driver mass because the radiator mass increases as the 4th power of the temperature and approximately inversely with the driver efficiency. Nevertheless, a decreasing efficiency would normally indicate that more driver modules are necessary to output the same $5 \mathrm{MJ}$, but this is not considered in the values quoted above.

\section{Table IV-2}

\section{Excimer Laser Processes}

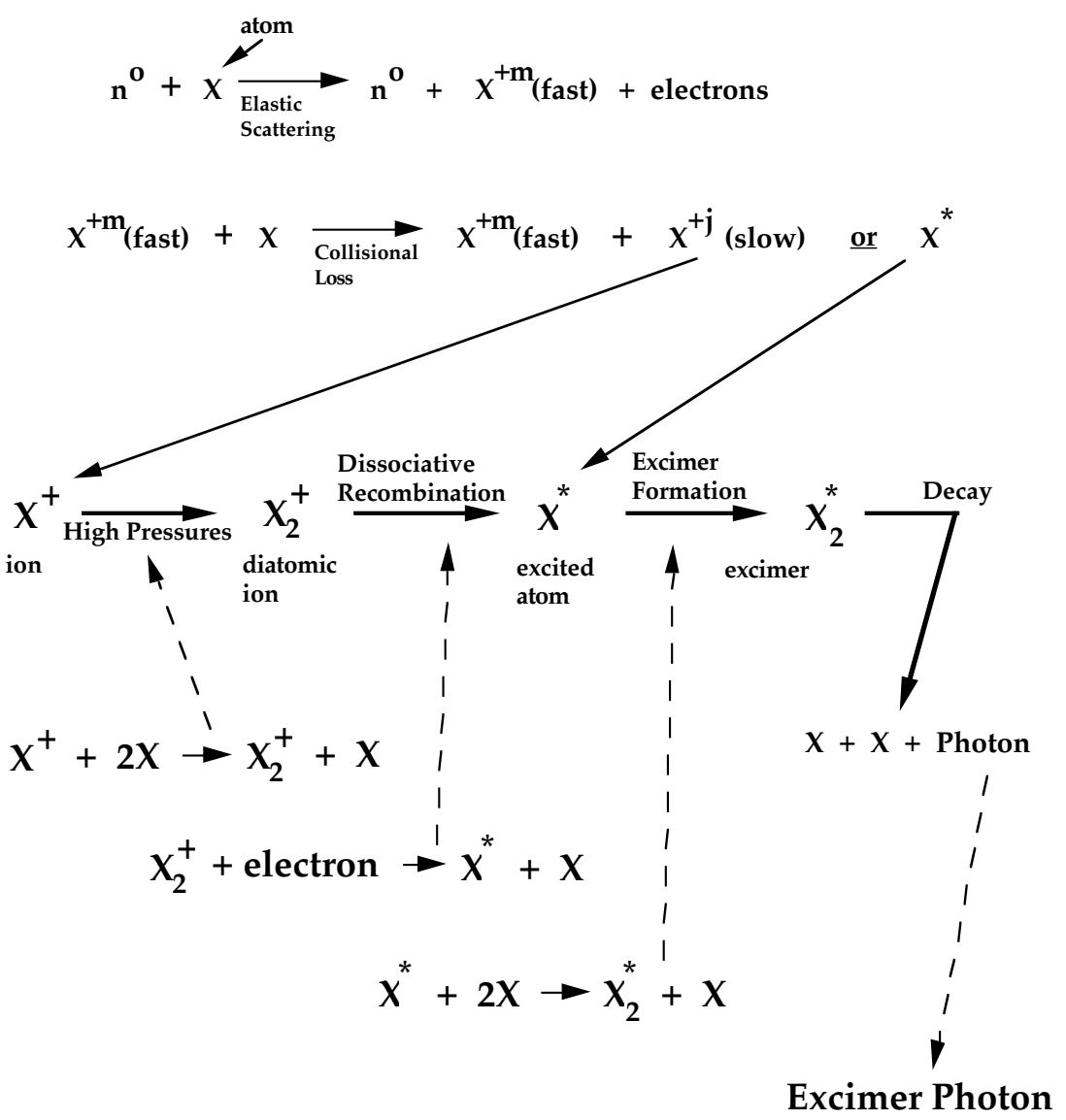

For excimer-laser parametric studies, we assumed that the driver mass (laser plus power conditioning subsystems) was $50\left(1+E_{d r}\right)$ tons, with the driver output energy $E_{d r}$ in MJ. The masses of the power-conditioning and radiators subsystems are discussed in Sections VI and VII, respectively. We assumed that the pulsed power requirements for the excimer laser pumps would be similar to those for KrF systems, namely, $600 \mathrm{kV}$ pulses 400 ns wide at repetition rates from 1 to $50 \mathrm{~Hz}$. The $400 \mathrm{~ns}$ value is appropriate for $\mathrm{KrF}$ systems, and is required due to the inherent internal losses of the $\mathrm{KrF}$ lasing medium. If such a long pumping time is utilized, hardware must be incorporated to compress the output laser pulse to the tens of nanoseconds required by the target. We made no attempt to detail the 
designs of these or any of the other driver subsystems (main oscillators, pumps, amplifiers, spatial filters, etc.).

\section{IV.5 Beam Line Optics and Focusing}

We envision the driver system to be composed of a many (at least 60 to 100) laser modules, with each module including equipment to generate, focus, and steer a beam toward one of the final turning mirrors. A critical factor for extended driver performance for an IFR is the lifetime of the beam-line optics. Although a KrF system operates at $1 / 4 \mu \mathrm{m}$ wavelength, both laser-target coupling and optics damage would dictate an optimum wavelength between $1 / 3$ and $1 / 2 \mu \mathrm{m}$, as provided by a DPSL. At $1 / 4 \mu \mathrm{m}$ and $1 \mathrm{~ns}$ pulsewidths, bare polished metallic mirrors are damaged above 5 to $6 \mathrm{~J} / \mathrm{cm}^{2}$, while mirrors coated with highly reflecting coatings are damaged above only 2 to $3 \mathrm{~J} / \mathrm{cm}^{2}$, using 1986 technology.$^{59}$ These damage thresholds increase for longer pulse-widths, scaling approximately according to the 0.4 power. ${ }^{60}$ Thus, for 30 ns pulse-widths, the above thresholds would increase by a factor of 4 . The thresholds also increase if the wavelength is increased, increasing by another factor of 2.5 to 3 if the wavelength is $1 / 2 \mu \mathrm{m} .{ }^{61}$ These thresholds are relatively independent of incident angle because the dominant damage mechanism is through defect sites that are essentially spherical. Damaged thresholds for coated transmitting (glass) optics (as opposed to mirrors) are generally somewhat higher. It is not known how any of the damage thresholds are dependent on the number of pulses for numbers above $10^{4}$ pulses, and we need at least $10^{8}$.

In this report, we assume that there are only two final turning mirror assemblies; however, future technological developments must determine whether the optimum number of final beams is one, two or many, and their spatial configuration, according to the achievable damage threshold at the pulse duration and wavelength used. If we assume a typical lifetime IFR damage threshold of 5 to $12 \mathrm{~J} / \mathrm{cm}^{2}$, which is 0.05 to $0.12 \mathrm{MJ} / \mathrm{m}^{2}$, then a 5 MJ output requires at least 40 to $100 \mathrm{~m}^{2}$ of optics. This means that each of two final turning mirrors must be at least 20 to $50 \mathrm{~m}^{2}$, and that a minimum of 40 to 100 driver modules is required for $1 \mathrm{~m}^{2}$ apertures. We will here consider $20 \mathrm{~m}^{2}$ final mirrors as the absolute minimum size, which requires materials development (see Sec. V.4).

The two final turning mirror assemblies shown in Fig. I-1 must be located opposite each other, at perhaps 179 degrees (or whatever angle is appropriate so that a laser firing that misses a target won't cause light to reflect up the opposite beam line and cause damage-but also at an angle that won't disrupt the illumination symmetry for the target). Each mirror assembly could be a single mirror or a ring of mirrors configured to illuminate the target with a hollowed cone of illumination less than is usually necessary for indirectdrive targets to have full gain. The use of a smaller beam cone angle will degrade the target gain somewhat, and this degradation must be taken into account in the systematics for VISTA if two-sided target illumination is used.

The two mirror assemblies, unlike the other optics, are exposed to the soft x-rays from each target firing. For a $5 \mathrm{MJ}$ driver and a target gain of 1000, with about $20 \%$ of the target energy going into x-rays (Eq. 8, Sec. III.6), these mirrors see a fluence of $0.1 \mathrm{MJ} / \mathrm{m}^{2}$ even if they are placed 25 meters from the target. Our own computer code for calculating x-ray deposition profiles, masses vaporized, and ablation momentum transfers indicates that this exposure is enough to ablate about $2 \mu \mathrm{m}$ of $\mathrm{Al}$ off each mirror every pulse, if the mirror were $\mathrm{Al}$, but the exposure would not even melt beryllium. Because it is imperative to avoid 
mass loss per pulse of any significance in comparison with the $50 \mathrm{~g}$ expellant employed per pulse, some metal like Be (with a large cohesive energy density ${ }^{1}$ and good thermal conductance) must be used for the front mirror faces, and not polymer highly reflective (HR) coatings. HR coatings will not withstand the x-ray thermal load, and may also be damaged by the integrated neutron fluence. Some reflectance loss may thereby result at 0.3 to $0.5 \mu \mathrm{m}$ wavelengths, but the loss in terms of net laser efficiency is negligible. Such reflectance loss at these final turning mirrors depends somewhat on wavelength, and is therefore a mild constraint on the final choice of driver wavelength.

Grazing-incidence metal mirrors (GIMMs) were proposed ${ }^{62}$ for the final optics for terrestrial IFE applications, but later damage studies discovered the above-mentioned fact that the damage threshold for metallic reflecting optics is relatively independent of incident angle. The use of GIMMs has therefore not been pursued, but instead, grazing-incidence liquid-metal mirrors (GILMMs) have received some attention. ${ }^{63}$ Such mirrors (e.g., liquid $\mathrm{Na}$ ) would require a centrifugal gravitational field similar to that already incorporated for VISTA, but could probably not keep a flat-enough figure in VISTA's vibrational environment. Future study could evaluate whether GILMMs have any merit for this application.

${ }^{1}$ The cohesive energy density is defined as the energy required per unit mass to heat the material from room temperature up through vaporization. 


\section{THRUST CHAMBER SYSTEMS}

The thrust chamber is the heart of the IFR engine, in which the charged plasma-debris energy from a target is converted into vehicle thrust. The chamber extent is defined by the envelope of particle trajectories followed by the target debris as it expands away from the target's firing position and interacts with a powerful magnetic field (Fig. V-1). As the debris interacts with the magnetic field, it is deflected rearward, creating a pulse of thrust with an effective interaction time with the spacecraft structure of roughly $50 \mu$ s.

Figure V-1: Magnetic Thrust Chamber Operation

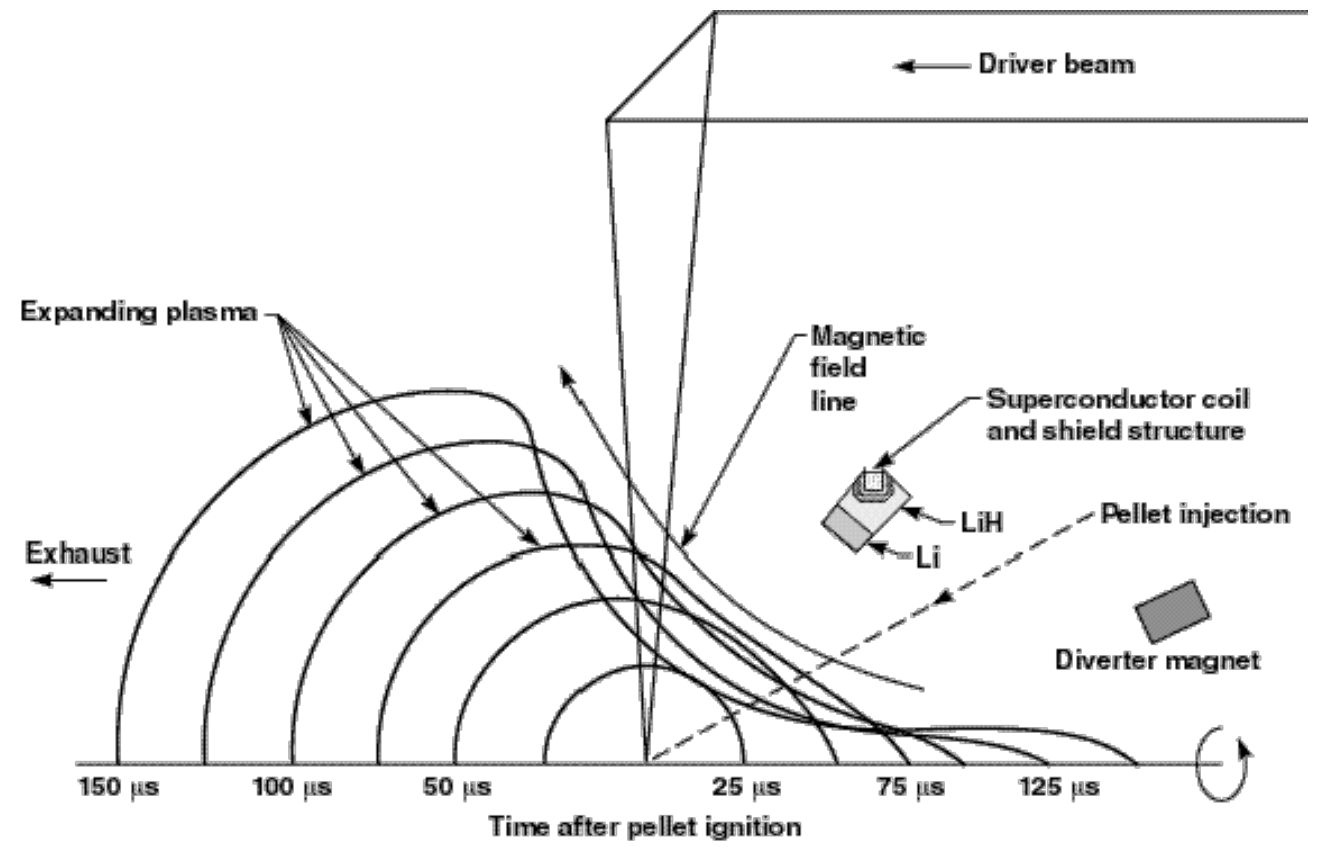

Orth art 1

The thrust-chamber system includes the evacuated region specified as the thrust chamber, the large superconducting magnet whose field determines the extent of the chamber, and all of the subsystems needed for the magnet coil: electrical charging circuitry, superconductor refrigeration equipment, neutron shields, shield coolant recirculating subsystem, shield x-ray ablation protection subsystem, and optional electrical power inductor pickup probe. It also includes any extra magnets (such as diverter magnets, if needed) to shape the exhaust plume for higher jet efficiency. 


\section{V.1 System Configuration and Basic Engine Operation}

The system configuration (Fig. V-1) and its overall operation are similar to those conceptualized by Hyde ${ }^{64}$. The biggest difference in the configuration is that the entire structure has been enlarged by a factor of two. This was done to avoid excessive ablation of the inner coil shield by the target $x$ rays (see below).

The operation of the thrust chamber is as follows. When the target is ignited by the laser beams, photons from the hot fusion core immediately ionize all of the expellant. Most of the more energetic photons (above about $100 \mathrm{keV}$ ) are able to penetrate all of the expellant and escape. These photons form an extremely short burst of energetic x-rays streaming out from the target location isotropically in all directions at the speed of light. Because the total energy content of these $\mathrm{x}$-rays is very small, they can be ignored.

Next to be emitted are the $14.1 \mathrm{MeV}$ neutrons, which also stream out isotropically from the burn region in all directions, but at one-sixth the speed of light. In reality, because many of the neutrons interact with the compressed target and expellant material, a distribution of neutron energies results, with the maximum energy being about $14.1 \mathrm{MeV}$. These neutrons carry away 50 to $60 \%$ of the DT fusion energy (they would carry away $80 \%$ based on a single reaction, but some interact before leaving the target -- see Sec. III.6). Consequently, they must not be allowed to impart energy to the magnet superconductor, or the superconductor would transition to its normal state and stop the engine. A thick coil shield is therefore placed on the target side of the coil inner windings to absorb the energy of all incoming neutrons (Fig. V-2 below). Based on solid-angle considerations, 3.93\% of the neutrons strike this shield, which must be cooled by a waste-heat radiator system. The rest of the neutrons continue on their way and leave the vicinity of the spacecraft in a time on the order of $1 \mathrm{~ms}$ (except for a small fraction which interacts with the final turning mirrors and the target injector outlet port). Soon thereafter, when the hot target thermal front burns its way through to the surface of the expellant, a burst of soft x-rays travels outward from the target site and interacts with the same equipment.

As the thermal wave burns through the expellant, before the emission of the burst of soft x-rays, the ionized expellant debris plasma begins propagating outward from the target isotropically in all directions as its thermal energy is converted into radial kinetic energy. The debris, being charged (i.e., conducting), does not allow any significant magnetic field to penetrate its boundaries. (Actually, the magnetic field lines do penetrate the surface of the plasma, but only to a depth to which surface currents are generated whose induced magnetic fields oppose the main field and inhibit its penetration into the plasma). The debris expansion thereby compresses the magnetic field lines, and this compression generates forces that oppose the motion of all debris traveling towards the magnet coil. When the generated magnetic pressure balances the plasma pressure, the debris is deflected rearward because the plane of the coil is forward of the target firing position (Fig. V-1). Thus, a significant fraction of the original plasma kinetic energy is transferred into magnetic potential energy stored in the field of the superconducting magnet, like the slowing of a ball thrown at a spring. After deflection, the stored energy is returned to the debris kinetic energy as the debris is pushed away from the magnet coils in the direction of the exhaust.

The impulse imparted to the magnet coil by the deflection of the debris plasma produces thrust. In a total elapsed time of order $100 \mu \mathrm{s}$, the debris has traveled rearward enough to allow the field lines to move back close to their original configuration, with the duration of the thrust pulse to the structure holding the magnet being essentially only a few 
to $10 \mu \mathrm{s}$. Note that this engine utilizes only that part of the fusion energy leaving the target site as debris kinetic energy; the target neutrons and $x$ rays thus constitute waste energy.

Hyde $^{65}$ calculated the debris trajectories shown in Fig. V-1 using a hydrodynamics program modified with a subroutine to determine the interaction of a highly conducting plasma with his particular design of superconducting magnet. We use a slightly different magnet design, but the basic operation is the same because the basic geometry is the same (just scaled in size by a factor of 2). Hyde assumed that the plasma would recombine (neutralize) by the time it exited the thrust chamber, thereby permitting complete decoupling of the debris from the magnetic field lines so that no residual plasma would spiral around the field lines and return to strike the spacecraft at another location. The validity of this assumption is unknown, and experimental tests are needed to verify its validity (see Sec. V.5).

\section{V.2 Superconducting Magnet}

The superconducting magnet creates the strong magnetic field that deflects the isotropically expanding conductive target debris plasma so that it exits the spacecraft in a small solid-angle range to provide thrust. The diameter and positioning of this magnet coil relative to the target firing location determine the geometry of the spacecraft (see Sec. IX.2). Although other options are available, we adopted the same relative geometry as optimized by Hyde ${ }^{66}$, but enlarged by a factor of two: the coil radius is $13 \mathrm{~m}$, with the plane of the coil placed about $11 \mathrm{~m}$ forward of the target firing position. Figure V-2 shows a preliminary conceptual design of the coil geometry in relation to its neutron-gamma-ray shield (Sec. V.3).

Hyde selected a $\mathrm{V}_{3} \mathrm{Ga}$ superconducting material embedded in a matrix of $\mathrm{V}$ and $\mathrm{Al}$, operating at $85 \%$ capacity, and cooled by liquid helium at $4.8 \mathrm{~K}$. Advances in the development of near-room-temperature superconductors ${ }^{67}$ suggest that it is highly likely that a suitable superconductor will be developed by the mid-21 $1^{\text {st }}$ century that can be cooled by liquid nitrogen or even warmer refrigerants. Further development is required, however, to ensure that both high magnetic field capability and zero resistance can be achieved simultaneously at these warmer temperatures. Use of warm superconductors would make both the mass of the refrigerator to cool the coil, and its power consumption, negligible in comparison with the other spacecraft dry mass.

Because of these anticipated developments, we chose to use a warm superconductor of unspecified material for the baseline VISTA coil design. This lowers the mass of the refrigerator, and helps offset the increased coil mass required by the larger dimensions. Due to the uncertainties in the choice of superconducting material, we did not design the refrigerator or the coolant-flow cycle or the coil winding configuration and cryostat/dewar. We did assume roughly the same $\left(60 \times 60 \mathrm{~cm}^{2}\right)$ cross section of coil windings in sizing the coil shield as Hyde, and thus assumed a capacity of $4.7 \mathrm{kA} / \mathrm{cm}^{2}$ for the superconductor matrix. The matrix consists of the yttrium-barium copper oxide wire or equivalent, with a copper wire core, plus a normal-conducting material into which the wire is embedded. The embedding restricts the wire's movement and thereby stabilizes the superconductor against transition [quenching] to its normal state by avoiding "flux jumps" that produce magnetic fields above the wire's critical field. 
Figure V-2: Preliminary Conceptual Design of the Superconducting Magnet and Magnet Shield

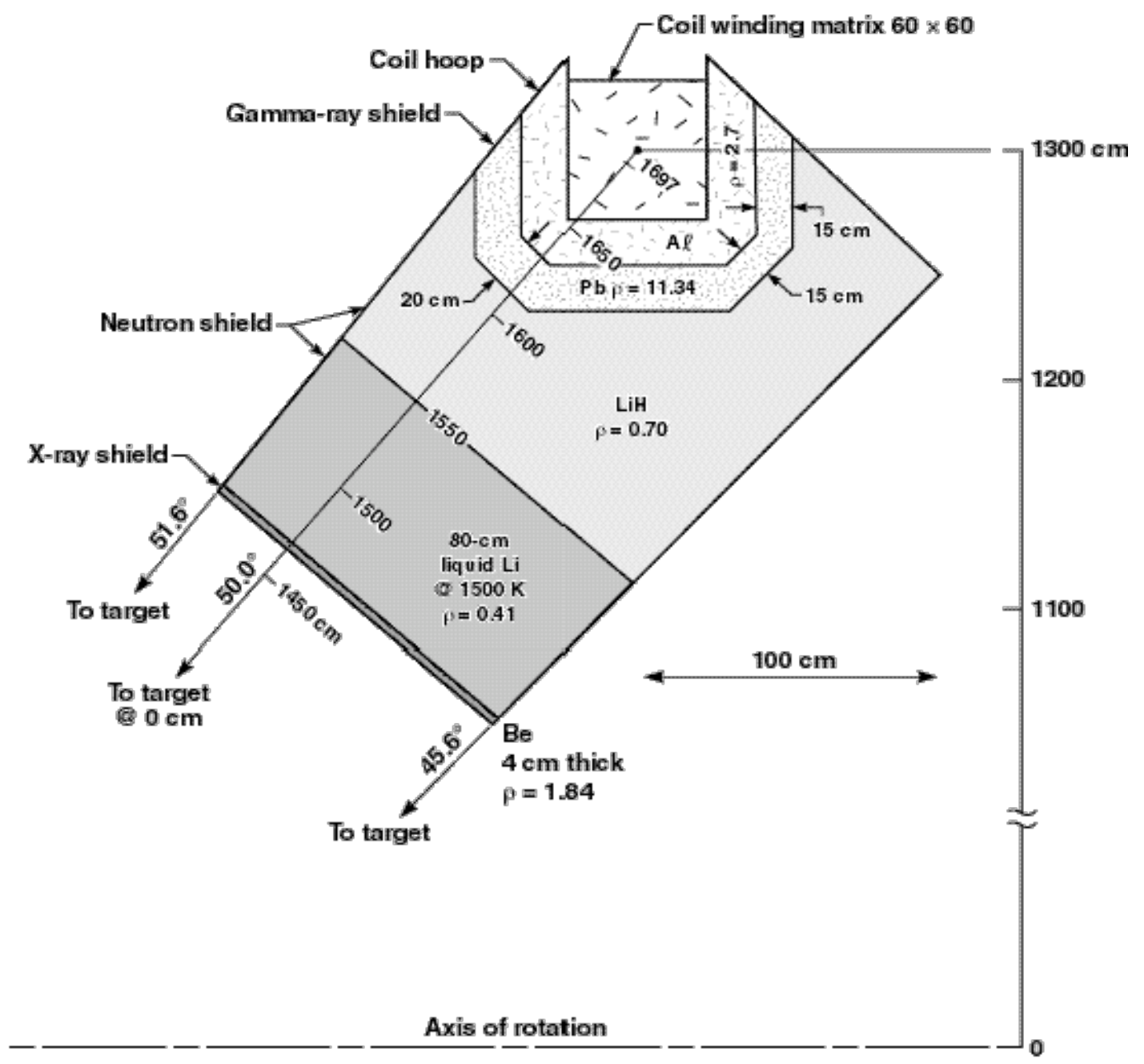

Orth art 2

Hyde's coil design used 22 MA-turns with a peak field of 15.8 Tesla and a total stored energy of about 6,500 MJ to deflect 1,280 MJ of debris. Since our maximum debris kinetic energy is 1,840 MJ, we increased the coil current-turns by the square root of the energy ratio. However, the larger coil has an inductance $(65 \mu \mathrm{H})$ about 2.4 times larger, and the current-turns can be decreased by the square-root of this factor. VISTA therefore uses 16.8 MA-turns, with a stored energy of about 9,200 MJ (still 5 times the maximum debris kinetic energy) and a peak coil field of 12 Tesla. 
C. Orth, UCRL-LR-110500

Table V-1 Assumed Magnet Coil Matrix Composition

$\left(\right.$ Density $=4.3 \mathrm{~g} / \mathrm{cm}^{2}$ ) For Fig. V-2 Design

\begin{tabular}{|c|c|}
\hline Element & Fraction by weight \\
\hline $\mathrm{V}$ & 0.487 \\
\hline $\mathrm{Al}$ & 0.315 \\
\hline $\mathrm{C}$ & 0.103 \\
\hline $\mathrm{Ga}$ & 0.079 \\
\hline $\mathrm{H}$ & 0.016 \\
\hline
\end{tabular}

Table V-2 Magnet Coil Parameters

\begin{tabular}{|c|c|}
\hline Parameter & Value \\
\hline Current-turns & $16.8 \mathrm{MA}$-turns \\
\hline Stored Energy & $9,200 \mathrm{MJ}$ \\
\hline Peak Field & $12 \mathrm{Tesla}$ \\
\hline Inductance & $65 \mathrm{\mu H}$ \\
\hline Radius & $13 \mathrm{~m}$ \\
\hline
\end{tabular}

Hyde's coil mass and structural composite mass were 8.7 and 8.5 tons respectively. At twice the coil radius, the values for VISTA could be quadruple those of Hyde. The values could be even larger if the coil current-turns were increased to reproduce Hyde's field values at his radii from the target, instead of preserving the ratio of stored field energy to plasma kinetic energy. For VISTA, a preliminary design of the magnet and shield (Fig. V-2) suggests that the masses of the coil and shield should in fact be about 216 tons and 505 tons, respectively (Table V-3). Although some materials development and advanced design might decrease the values in Table V-3, we shall use them here to be conservative, assuming that the 505 tons for the shield includes its thermal system. We also assume that the coil will have a refrigeration subsystem and a structural support subsystem separate from the main vehicular trusses which all together account for about 18 tons.

A superconducting coil requires an electrical charging circuit with an auxiliary power source to energize the magnetic field upon engine startup. We did not design the charging circuitry, which is existing technology, but typical charging times are on the order of several hours for a $1 \mathrm{MW}$ charging system.

One precaution with such a strong magnet onboard is to avoid the use of materials or equipment nearby that might be affected by a steady (essentially DC) magnetic field. Because the dipole field decreases with distance $d$ away from the magnet as $d^{-3}$, there should be little worry for the crew and crew systems. On the other hand, equipment located closer to the magnet (target injector, laser systems, etc.) must be designed to avoid magnetic influences. 
C. Orth, UCRL-LR-110500

Table V-3 216-ton Magnet and 505-ton Shield Composition Corresponding to Fig. V-2

\begin{tabular}{|l|c|c|c|}
\hline \multicolumn{1}{|c|}{ Material } & $\begin{array}{c}\text { Density } \\
(\mathbf{g} / \mathbf{c m} 3)\end{array}$ & $\begin{array}{c}\text { Layer Thickness } \\
(\mathbf{c m})\end{array}$ & $\begin{array}{c}\text { Approx. Mass } \\
\text { (metric tons) }\end{array}$ \\
\hline $\mathrm{Be}$ & 1.84 & 4 & 8 \\
\hline $1500 \mathrm{~K}$ liquid Li & 0.41 & 80 & 36 \\
\hline $\mathrm{LiH}$ & 0.70 & $\sim 120$ & 101 \\
\hline $\mathrm{Pb}$ & 11.34 & 15 to 20 & 360 \\
\hline $\mathrm{Coil}$ Al hoop & 2.7 & 20 & 90 \\
\hline Coil Winding Matrix & 4.3 & 60 & 126 \\
\hline Totals & & & 721 \\
\hline
\end{tabular}

\section{V.3 Coil Shield}

A shield composed of $\mathrm{Li}, \mathrm{LiH}$, and $\mathrm{Pb}$ sections patterned after those of $\mathrm{Hyde}^{68}$ lies on the target side of the superconducting coil to minimize the heat flux from target neutron emissions into the superconducting medium (Fig. V-2). For high-performance operation, typically $7 \mathrm{GW}$ of neutrons (and gamma rays) must be absorbed by this shield and removed by the coil waste-heat rejection subsystem, assuming a fractional solid angle of the shield as seen at the target of 0.0393 . Lithium is a good choice as a neutron absorber because it is low- $Z$ and can act as the heat-transfer fluid for the waste-heat rejection system. Lithium-hydride can be used for the rear portion of the shield because its higher density and hydrogen content make it a more effective neutron absorber, even though it can not withstand high temperatures and remain solid. Lead is used between the $\mathrm{LiH}$ portion of the shield and the coil to stop gamma rays created in the lithium material by $(\mathrm{n}, \gamma)$ reactions.

Because the mean free path of $14-\mathrm{MeV}$ neutrons in $\mathrm{Li}$ is about $0.15 \mathrm{~m}$, Hyde had to include approximately $1 \mathrm{~m}$ of $\mathrm{Li}$ and $1 \mathrm{~m}$ of $\mathrm{LiH}$ to reduce the heat load sufficiently for lowmass refrigeration subsystems to maintain $5-\mathrm{K}$ superconducting media. Use of warm superconductors reduces the requirement to about $3 / 4$ these dimensions. Without knowledge of what the superconducting wire will ultimately be, we estimate that the coil's minimum dimensions will be $60 \times 60 \mathrm{~cm}^{2}$ in square cross section. Such a coil subtends $2 \%$ of the solid angle at the target $17 \mathrm{~m}$ away. We assumed that this must be expanded to about $4 \%$ for the Li material to avoid neutrons scattering into the superconductor from the edges of the shield, and to increase the tritium breeding ratio (Section III.9). The fractional solid angle of the shield as viewed at the target in Fig. V-2 then becomes [Cos(45.6)-Cos(51.6)]/2 $=0.0393$. This shield includes 137 tons of $\mathrm{Li}$ and $\mathrm{LiH}$. To this we must add a $15-\mathrm{cm}$ thickness of lead for a gamma-ray shield at 16.5-m radius, whose mass is 360 tons. The total shield mass, including supports and 8 tons of Be as an x-ray shield (see next Section), is therefore about 505 metric tons.

The thermal control system for the shield uses the $\mathrm{Li}$ in its liquid state operating at $1500 \mathrm{~K}$ as the transfer fluid from the coil to the coil waste-heat radiators. The heat arises from neutrons (and gamma rays) from the target reacting in the Li fluid. We ignore here the design details of just how the $1500-\mathrm{K}$ material is thermally isolated from the magnet coil matrix, which of course must remain in its superconducting state. 
One potential problem with the use of a conducting-metal shield is a possible dissipation of the distorted dipole magnetic field through radiation or induced-current losses. The latter would also constitute an added heat source for the shield radiators. Future studies must reveal whether or not these issues are important. If resonant dipole oscillations ever become important, power losses can be reduced by injecting targets antiphased with the dipole fluctuations.

The optimum size of the shield is a trade-off between excessive mass for a large shield, and insufficient tritium breeding and increased neutron-gamma irradiation of spacecraft components and crew quarters for a small shield. Specifically, Hyde assumed 0.030 was a sufficient solid angle for his geometry, but expanded it to 0.055 to enhance tritium breeding when Monte Carlo neutronics codes indicated that the tritium breeding ratio would be only $54 \%$ for a solid-angle fraction of 0.030 . We have not performed additional calculations for the VISTA design, and leave to a future study just how these trade-offs would best be optimized. Nevertheless, Sahin, et al. ${ }^{69}$ have calculated the neutron and gamma-ray heating for our shield design and found it to provide adequate protection for the superconducting material for total fusion yields up to at least $17.5 \mathrm{GW}$. They recommend, however, that the center-line of the magnet within the shield be shifted from 50.00 to 50.15 degrees in Fig. V.2 to reduce peak heating in a few isolated spots within the shield.

\section{V.4 X-Ray Ablation Protection for First Wall \& Final Mirrors}

Target x-ray emissions do not heat the Li inside the coil shield due to their short ranges, but they can cause serious erosion of the front metallic surface of the shield's Li containment vessel and for the front faces of the final laser turning mirrors. Without considerable design effort to minimize this ablation, the mass loss per pulse can greatly exceed the expellant employed per pulse, and mission performance is severely degraded. Such ablation therefore sets one of the most important design constraints on the size of the superconducting magnet, and hence on the entire spacecraft. This fact has not been appreciated by any previous author in this field.

Using our own computer code for calculating $\mathrm{x}$-ray deposition profiles, masses vaporized, and ablation momentum transfers, we calculate that the ablation of hundreds of grams of material per pulse off the inner coil shield from each 7500-MJ target fired in the Hyde geometry can be avoided if 1) the shield inner surface is much further away than about $10 \mathrm{~m}$ from the target, and 2) the material employed for the front surface is one with a large cohesive energy density, like carbon or beryllium. The last point can be understood by noting that most materials have a similar cross section for x-ray absorption in terms of $\mathrm{g} / \mathrm{cm}^{2}$, so the material that is best is the one that can absorb the most energy per gram before it melts or vaporizes. Moreover, our calculations suggest that it is impossible to reduce Hyde's vaporization mass loss through protective schemes employing techniques to coat the inner surface with expendable liquids (e.g. by wicking), or to puff gas in front of the surfaces to absorb the target emissions, because such techniques require about the same mass in the protecting agent as would be vaporized directly from the surface. These techniques would therefore degrade jet efficiency because they do not effectively enhance engine thrust, and eject low-velocity material at large solid angles. In addition, to accommodate the optional emf-generating coil power-conversion system discussed in Sec. VI.3, the inner coil surface must be metallic (i.e. a good electrical conductor). The material must also be a good thermal conductor, to facilitate heat removal. We therefore use 
beryllium metal for VISTA. As added protection, we also plan to ripple or "spike" the surface to increase the surface area and thereby decrease the heat loading per unit of material area.

We also suggest using some form of polished beryllium as the surface of the final laser turning mirrors, and to place them at a distance of $40 \mathrm{~m}$. (The soft $\mathrm{x}$ rays from a 7500-MJ target would still ablate at least $1 \mu \mathrm{m}$ of $\mathrm{Al}$ at $25 \mathrm{~m}$.) Distances greater than $40 \mathrm{~m}$ would reduce the thermal loading and should therefore be considered as well, for we know of no limiting constraint on the placement of these mirrors-except for their mass, which would have to increase for larger distances. Unfortunately, pure polished beryllium has a reflectance of about $43 \%$ at 0.3 to $0.5 \mu \mathrm{m}$, so use of beryllium requires a development project to obtain a surface having both high heat of vaporization and high reflectance. Stated differently, the final choice of driver wavelength may depend partly on the reflectivity of Be: a reflectivity too low will require a larger driver mass, and may introduce complexity and mass in heat-rejection systems. Activation of the beryllium from target neutron emissions should not create a radiological problem for the crew and should not significantly affect the structural integrity of the beryllium. On the other hand, in the unlikely case that grazing-incidence liquid-metal mirrors (GILMMs) can be developed for this dynamical environment (complete with vibration), then reflectivity is not a problem.

\section{V.5 Target Debris Plasma Physics}

Although one might at first view the expansion of the target debris plasma and its interaction with the magnetic field as a simple adiabatic expansion of a hot plasma into a vacuum and the deflection of this plasma by a huge magnetic field, closer inspection reveals that rather complicated phenomena are involved. We have not taken the effort to understand much of the physics of these phenomena, even though some aspects are crucial to the engine's basic operation. A future study must therefore more fully address these important issues.

The most important issue needing further experimental understanding is the time history of the conductivity of the debris plasma. This plasma must be sufficiently conductive to expel the magnetic field and thus allow redirection of the plasma rearward, or the engine will not operate. Initially, just after termination of the fusion burn, the plasma temperatures vary from a few to tens of $\mathrm{eV}$ at its surface to hundreds of $\mathrm{eV}$ on its interior, and is therefore highly conductive. After 1 to $10 \mu \mathrm{s}$, when the plasma has expanded to a radius of roughly $1 \mathrm{~m}$, the thermal wave from its interior has already propagated outward and reached the surface, releasing a burst of soft x-rays, and the plasma has cooled down to the order of $1 \mathrm{eV}$. This cooling process involves the conversion of the thermal energy into radial kinetic energy, thus providing the high engine specific impulse (debris speeds near $1 / 4 \mathrm{~m} / \mu \mathrm{s}$ ). The cooling process does not depend on external radiation (the hydrogen expellant has no bound electrons when ionized). Even at $1 \mathrm{eV}$, the plasma conductivity is still within about 3 orders of magnitude of metallic conductivities. Although there are questions of plasma collisionality and plasma instabilities, the important question is how cold the plasma will become as it continues to expand, while continually being "reheated" through its own recombination. Sufficient cooling would of course lower the conductivity enough that some magnetic field could penetrate the outer portions of the debris during the deflection process, thus lowering the efficiency of the engine through energy lost during field decoupling in the plume. That is, the outer fringes of the exhaust volume would 
suffer a kinetic-energy loss to supply the energy necessary to extract the field lines, and this momentum loss would lower the effective jet efficiency. In addition, there is the possibility that charged particles that remain in the field-penetrated regions may tend to spiral around the field lines and eventually impinge on spacecraft structures. Future studies may want to investigate the value of attempting to raise the plume's surface conductivity using blasts from other lasers.

Decoupling of the plasma from the magnetic field in the plume depends on the beta of the plasma, which is the ratio of the particle pressure and the magnetic field pressure (i.e. the ratio of the kinetic energy density of the plasma and the energy density of the magnetic field):

$$
\text { Beta }=n k T /\left(B^{2} / 2 \mu_{0}\right)
$$

At the point of deflection, beta is near unity. In the plume, B is decreasing as $\mathrm{r}^{-3}$, while the particle energy is essentially constant, so beta increases rapidly. The plasma should therefore decouple from the field with high efficiency, but detailed calculations proving this are still lacking. Low betas would of course inhibit decoupling, thus causing drag, decreased jet efficiency, and unwanted particle flow forward around the field lines and onto spacecraft structures.

Zakharov, et al. ${ }^{70}$ have confirmed the general features expected for the expansion of a plasma in a dipole field through initial calculations and experiments. If future moredetailed studies indicate that plasma decoupling does cause excessive drag, one idea that has been proposed is to add cold expellant to the exterior envelope of the exhaust as it exits the thrust chamber. This is generally not a good idea, however, because the resulting layered plume then has a less conductive edge, which would promote further magnetic field penetration with subsequent increased drag; moreover, a non-thermalized exhaust component would reduce jet efficiency. Some investigators have also proposed using this hybrid-plume technique to control exhaust velocity in order to optimize propulsive efficiency (the ratio of vehicle power to engine power) by making exhaust velocity match vehicle velocity, but this would work only if the cold and hot exhaust components can fully thermalize-otherwise the jet efficiency is reduced. Techniques for plume velocity modulation and plasma tuning at high temperatures $(800 \mathrm{eV})$ have been extensively studied by F. Chang-Diaz of NASA, and by T. Yang and W. Krueger of the MIT Plasma Fusion Center. ${ }^{71}$ A more efficient way to control plume velocity, therefore, is simply to vary the mass of the expellant.

We here ignore the possibility of Rayleigh-Taylor and other instabilities in the plasma flow because we believe that the time scale for the instabilities is much longer than the transit time of the plasma through the engine. Total transit of the debris through the thrust chamber takes 100 to $150 \mu$ s. Detailed calculations of instabilities, however, are lacking. In the unlikely event that future studies indicate that instabilities are important, quench margin may have to be added to the design of the superconducting magnet. 


\section{V.6 Plume Formation and Plasma Collection Efficiency}

The debris exiting the spacecraft from the thrust chamber and decoupling from the magnetic field constitutes the exhaust. The plume geometry is determined by the distribution of this exhaust in solid angle. This distribution is determined by the interaction of the debris with the magnetic field, and was calculated by Hyde. ${ }^{72}$

If future studies determine that it is necessary to increase jet efficiency by further restricting the solid angle of exhaust exit, the plume can be shaped by employing other magnets. Additional magnets, however, would involve additional dry mass, primarily in support structure to resist the huge forces between magnet coils, and the additional neutron coil shields that would be required to protect the superconducting media from thermal influx. The additional coils and shields would also constitute mass not situated behind the coil shield for the thrust-chamber magnet, as viewed from other spacecraft structure, so target neutrons could scatter in the mass of these other coils and shields and produce a neutron background for the entire spacecraft. Thus, although plume-shaping coils are an option, their use would complicate the spacecraft design.

The plasma collection efficiency of the thrust chamber with just one coil was calculated by Hyde to be $64 \% .^{73}$ This value not only includes the loss of some plasma forward along the axis of the magnetic field, but also the effect of projecting all exiting debris momenta along the single rearward thrust vector. This efficiency can be compared with a maximum value of $75 \%$, which is obtainable only when the thrust chamber acts like a hypothetically perfectly reflecting paraboloid. The $64 \%$ can be increased very slightly by moving the target somewhat further away from the plane of the magnet coil, because Hyde reduced this distance (relative to the optimum distance) to enhance tritium production in the lithium coil shield. We will adopt the conservative value of $64 \%$ for VISTA.

\section{V.7 Jet Efficiency}

The physics of the plasma dynamics in the magnetic thrust nozzle of an IFR is not fully understood, but certain qualitative comments can be made. The jet efficiency of an IFR is

$$
\eta_{\text {jet }}=P_{\text {jet }} / P_{\text {input }}
$$

where we take the input power to be the sum of the driver power and the power released by the fusion target, $P_{\text {input }}=P_{d}+P_{\text {target }}=P_{d}(G+1)$, and the jet power is defined by

$$
P_{\text {jet }}=F^{2} /(2 d m / d t)
$$

Here $\mathrm{dm} / \mathrm{dt}$ is the total rate of mass flow from the propulsion system and $\mathrm{F}$ is the magnitude of the directed propulsive force or thrust exerted by the propulsion system. In a traditional rocket motor, $\mathrm{dm} / \mathrm{dt}$ is the propellant mass flow rate, but in general, $\mathrm{dm} / \mathrm{dt}$ must also include the flow of any non-propellant masses required for operation of the propulsion system (such as non-recovered target radiation shields). Note that the definition of specific impulse, using these variables, is

$$
\mathrm{I}_{\mathrm{sp}}=\mathrm{F} /(\mathrm{dm} / \mathrm{dt})
$$


which has the units of velocity in MKS units (but pound-force-seconds per pound-mass in English units). This is the reason why the term effective exhaust velocity is sometimes used instead of specific impulse, but specific impulse is equal to exhaust velocity only for a perfect mono-velocity beam of expellant fluid.

Several loss mechanisms have been identified that reduce jet power, and hence jet efficiency. The best understood of these mechanisms is the hydrodynamic loss caused by the imperfect collimation of the expanding plasma debris. As we mentioned above, not only is there a distribution of exiting angles scattered about the direction of thrust, but there is a small loss of plasma forward along the axis of the superconducting magnet. Hyde ${ }^{74}$ addressed these effects based on the assumption of perfect conductivity for the plasma (no magnetic field penetration of the plasma), and determined a loss in jet power of near $59 \%$ (that is, $1-0.64^{2}$ ). For comparison, a specularly reflecting hemisphere deflecting the plasma debris in a manner similar to that employed in the Daedalus study ${ }^{75}$ would lose $75 \%$ of the jet power.

Diffusion of the magnetic field into the plasma prior to plasma-field decoupling also causes a loss in jet efficiency, because the plasma is not perfectly conducting. A qualitative measure of this loss can be obtained by the ratio of the transit time of the plasma through the thrust chamber and the characteristic time for magnetic field diffusion into the plasma. The latter is given by

$$
t=\left(\mu_{0} L^{2}\right) / h
$$

where $\mu_{0}$ is the permeability of the vacuum ( $4 \pi \times 10^{-7}$ in MKS units), L is the scale length of the spatial variation of the magnetic field (a few meters), $h$ is the (Spitzer) resistivity of the plasma,

$$
\mathrm{h}=0.05 \mathrm{~T}_{\mathrm{ev}}{ }^{-3 / 2} \mathrm{ohm}-\mathrm{cm}
$$

where $\mathrm{T}$ is the electron temperature of the plasma. If the plasma temperature were to remain at its cooled state of $1 \mathrm{eV}$ even though it expanded beyond a radius of about $1 \mathrm{~m}$, the resulting time $t$ of about $250 \mu$ s would be comparable to the 100-150 $\mu$ s transit time through the thrust chamber. Thus, the IFR operates in a realm where the details determine whether the plasma conductivity is high enough to keep the magnetic field out. As indicated above, a future study must address these details. Here, we will arbitrarily assume that the diffusion loss is about $10 \%$. Due to the uncertainty in this value, we will not discuss in detail what happens to the plasma penetrated by the magnetic field. Some of this plasma will eventually separate from the magnetic field by ambipolar diffusion and exit the spacecraft in arbitrary directions. Some may remain trapped in the field and interact with spacecraft structure.

Lost thermal energy stored in the ionization of the expellant as the exhaust departs from the vicinity of the spacecraft is one frozen-flow loss that is potentially significant for low exhaust velocities. For our system, however, operating with a typical exhaust velocity of $270,000 \mathrm{~m} / \mathrm{s}$, the kinetic energy of each $\mathrm{H}$ expellant ion is about $400 \mathrm{eV}$, while the ionization energy is $13.5 \mathrm{eV}$. Low recombination rates would thus imply a loss of $3 \%$ at most. However, we anticipate high recombination rates by the time the exhaust is exiting the thrust chamber (even though this may cause plasma drag as explained in Section V.5), so we will ignore ionizational losses. 
Thermal radiation from the plasma can cause losses in jet power, but not for hydrogen until recombination occurs, because there are no bound electrons in the plasma state. This is one advantage for using a hydrogen expellant. Jet power can also be lost due to a nonmono-energetic exhaust velocity distribution. Such a velocity distribution can result directly from the engine operation, or indirectly through multiple ion species of widely different atomic masses (and therefore different velocities). In the IFR case with hydrogen expellant and no hybrid plume components, only the dispersion in velocity distribution is important. We did not determine the nature of this dispersion for an IFR, however, because the dispersion of velocities of the ions exiting the target location is modified by interaction with the magnetic field to form a more mono-velocity distribution. The target dispersion is roughly a factor of 2, so the final dispersion should typically be much less than a factor of 2 in velocity. A linear velocity distribution over a relative velocity range of 1.0 to 1.5 would lead to a loss of only $1.3 \%$ in jet efficiency.

Another consideration is whether the spacecraft structure absorbs some of the impulse vibrational energy from the repetitive target firings, thereby causing a decrease in the effective jet efficiency. Preliminary calculations, however, suggest that this is a negligible loss $(\mathrm{Q}<<1)$.

The above losses in jet power total at least $68 \%$, but a lower value is appropriate for a more optimum placement of the target in relation to the magnet coil plane, or if additional (but perhaps impractical) plume-shaping coils are employed. For VISTA, we originally assumed a total loss of $66 \%$ and thus a jet efficiency of $(1.00-0.66)$ or $34 \%$ so that the momentum efficiency of the engine (which is the square root of the jet efficiency) was 0.583 (i.e., 0.6). However, with the later, more-accurate system descriptions (see Sec. XI.2), the jet efficiency dropped to $32 \%$.

\section{V.8 Inductor EMF Power Conversion Option}

One option for power conversion is the use of a conducting path on the inside of the coil shield so that, when the debris interacts with the magnetic field and compresses the field lines, these lines cross through the conducting path and generate an emf. This emf can be utilized as discussed in Sec. VI.3 to generate power for the driver. The advantages of such a system are a great reduction in mass and improved efficiency of the powerconversion system in comparison to those for a Rankine system.

The most suitable choice for the conducting path is the interior surface of the coil shield itself, because the emplacement of an additional coil here would complicate the shielding and create huge structural forces between the main magnet coil and any physically separate pickup coil. The choice of beryllium as the interior shield liner for ablation protection is commensurate with the conducting requirements for the conducting path. Thus, the ablation protection system can serve both functions with no additional mass required.

\section{V.9 Blanket Options}

Another concept for power conversion is the use of spherical sectors purposely placed in the path of the target neutrons at about the same radius (or perhaps slightly larger radii) from the target than the coil shield. These sectors would have an interior beryllium ablation liner, just like the coil shield, and would contain liquid lithium for heat transfer (see Sec.

VI.1). Such systems would act much like a coil shield that subtends much more than $4 \%$ of 
the total solid angle (see Fig. V-2). They are referred to as blankets (because of the analogy with similar systems in terrestrial IFE facilities). The purpose of these blankets is to absorb more neutron energy, and convert the energy to power suitable to operate the driver.

The best location for the blankets depends on their subtended angle. Small blankets

could in effect be extensions of the coil shield, but larger systems could be located extending from the coil shield towards the coil axis. One constraint is to avoid neutron scatter towards other spacecraft systems, so blankets must be nearly as thick as the coil shield. As such, blankets are very massive, at least 30 tons for every $1 \%$ of solid angle (and 20 to $50 \%$ would be required!). This is just one feature that makes blankets vastly inferior to the inductor coil option.

\section{V.10 Shock Isolation}

Significant impulse is imparted to the coil structure only during a few meters of debris transit around the place of peak field compression. Engine impulse therefore lasts only on the order of $10 \mu \mathrm{s}$. A NASTRAN computer analysis of the conical spacecraft structure indicated that special shock isolation of spacecraft components from the magnetic drive coil impulsive shock loading is not required. The mass of the drive coil itself and the compliance of the surrounding support structure form a sufficient mass-spring momentum absorber to sharply attenuate the drive pulse peak loads. Several meters out along the conical shell away from the drive coil, only an averaged acceleration is seen by the structure because the effective spring-constant time of the structure is much longer than the inverse target repetition frequency of roughly $1 / 30 \mathrm{~Hz}=30 \mathrm{~ms}$. 


\section{POWER SYSTEMS}

The power systems must be capable of supplying pulses to operate the laser driver plus approximately $1 \mathrm{MW}$ of auxiliary dc power. The power for the driver depends inversely on the efficiency of the driver, and is a maximum of $2.5 \mathrm{GW}$ at $30 \mathrm{~Hz}$ for a $6 \%$-efficient driver, as originally envisioned for VISTA, but only $1.25 \mathrm{GW}$ at $30 \mathrm{~Hz}$ for VISTA's 12\%-efficient DPSSL driver. The power systems consist of two subsystems: a power-conversion system to extract power from the fusion process, and a powerconditioning system to form the proper electrical pulse shapes to operate the driver and other electrical equipment.

\section{VI.1 Overall Concepts}

Power to operate spacecraft systems must come from the fusion reactions because the gigawatts of average power required cannot be obtained with solar arrays of practical size (nor do we want VISTA to be dependent on solar illumination). The total power available from the fusion reactions is up to 100 times the required electrical power. The emphasis in the design of the power systems is thus how to extract a small part of the fusion power using a system of minimal mass.

The mass of the power system is in turn very dependent on the technique used to obtain the power, and the available techniques depend on the timing pulse widths considered. For example, the fusion reactions offer a 10- $\mu$ s time scale of the fusion impulse in the thrust chamber as well as an essentially dc pulse available by absorbing the neutron and / or x-ray emissions as heat energy in either the coil shield or an external blanket absorber. To use the thrust-chamber pulse, the power systems must convert the 10- $\mu$ s time scale to the millisecond pulse widths required by the laser diodes used to pump the amplifier gain media for a DPSSL driver. This can be done directly, or by going through a dc stage. If the system uses neutron depositions in an external blanket, the technique would force the use of a dc heat stage. Any system requiring conversion through a dc stage will generally be more massive than a system which merely extends the $10-\mu$ s scale by a factor of 100 and stays in the pulsed domain.

The pulse width required by the driver is also important. If a KrF driver were considered instead of a DPSSL, for example, the short relaxation times of the $\mathrm{KrF}$ lasing state would require input pulses with a width of only $~ 400 \mathrm{~ns}$. Compressing the time scale of the 10- $\mu$ s thrust-chamber pulse to $400 \mathrm{~ns}$ might require more complicated circuitry than lengthening it to $1 \mathrm{~ms}$ for a DPSSL. Although future developments in excimer lasers might increase the required pulse lengths for practical excimer lasers, we will here assume that 1ms pulses will be required, as for a DPSSL. (The time scale for a DPSSL might even be longer than $1 \mathrm{~ms}$, depending on the development of crystal gain media with longer storage lifetimes.)

The primary objective for an IFR power-conversion system is thus to extend the few to 10- $\mu$ s pulse period of the thrust impulse to get the 1-ms pulse widths needed for the laser pump diodes for a DPSSL. Two overall systems concepts were considered for such powerconversion in VISTA: 1) a Rankine system utilizing the dc waste heat deposited in the superconducting magnet coil's lithium shield by target neutrons and gamma rays, and 2) an 
inductor-coil option that generates an emf through magnetic induction upon compression of the coil's magnetic field by the expanding target debris plasma.

The Rankine system starts with a dc heat source created as the lithium shield integrates incident neutron energy. It uses current technology (and is therefore a good backup option) and was designed in detail. Unfortunately, the Rankine system is only $20 \%$ efficient if optimized for minimal mass. As a result of both the dc nature of the conversion (which necessitates a massive electrical storage system) and the system inefficiency (which increases the mass in waste-heat radiators), the mass of the Rankine system is over 1,000 tons. Use of the Rankine system would in fact increase the dry mass of the spacecraft by more than $50 \%$ relative to the dry mass that results for use of an induction system. This is unfortunate, because the thrust chamber utilizes only the debris-plasma target emissions and treats the absorbed neutron/gamma-ray energy that heats the lithium in the coil shield as something to expel as waste heat, whereas the Rankine system would use a portion of these other target emissions that are simply radiated away as waste heat.

The inductor system, originally proposed by Hyde, ${ }^{76}$ uses the inherent time scale of the target debris emissions, and therefore involves minimal mass for the conversion system. The total mass of the power system is therefore determined more by the associated power-conditioning system. In particular, if the inductor power is rectified so that capacitative storage is required, the power-conditioning mass will be at least 100 tons. Although this mass is much less than that for a Rankine system, the objective is obviously to design an inductor conditioning system that uses efficient pulse expansion (and hence no dc stage) to achieve minimal added system mass. We have not developed such a design, so we leave that as a task for a future study. To be conservative, the baseline option for VISTA is the inductor-coil power-conversion system with a capacitative-based power-conditioning subsystem. The total mass of this system is about 115 tons.

It is very important to note that the induction system extracts power that would otherwise be used for thrust, whereas the Rankine system does not. The induction system is therefore practical only for large target gains, because only for such gains is the debrisplasma energy very large in comparison with the energy $\left[\mathrm{E}_{\mathrm{dr}} /\right.$ (driver efficiency $\mathrm{x}$ inductorpower-conversion efficiency)] required for the driver input.

Utilization of the fusion reactions for power conversion obviously necessitates the use of another electrical power generation system to start the fusion engine. We have not designed this engine startup system in detail, but its design is not critical, and is addressed in Sec. VI.7. 


\section{VI.2 Overall Power Flow}

Figure VI-1 shows the overall power flow for VISTA for a piloted Mars mission with target gain $\mathrm{G}=1500$ (advanced technology) using the inductor power system and maximum reprate $(30 \mathrm{~Hz})$. Note that $207 \mathrm{GW}$ of the $225 \mathrm{GW}$ of fusion power are simply radiated to space! The neutron-multiplication factor of 1.15 adds $0.74 \mathrm{GW}$ of waste heat in the coil shield A little more than $1 \%$ must be recycled to operate the driver through the induction power system. The jet efficiency is $18.86 /(52.81+2.50)=34 \%$ here, although $32 \%$ is more precise for the updated system descriptions of Sec. XI.2. Figure VI-2 shows the power flow using a Rankine system, which optimizes at $12.5 \mathrm{~Hz}$ and a jet efficiency of $35.7 \%$. The energy-multiplication factor of 1.15 is described in Sec. VI.5.

Figure VI-1: Overall Power Flow (in GW) for the Advanced-Technology Mission to Mars Using the Inductor Power System and Maximum Reprate

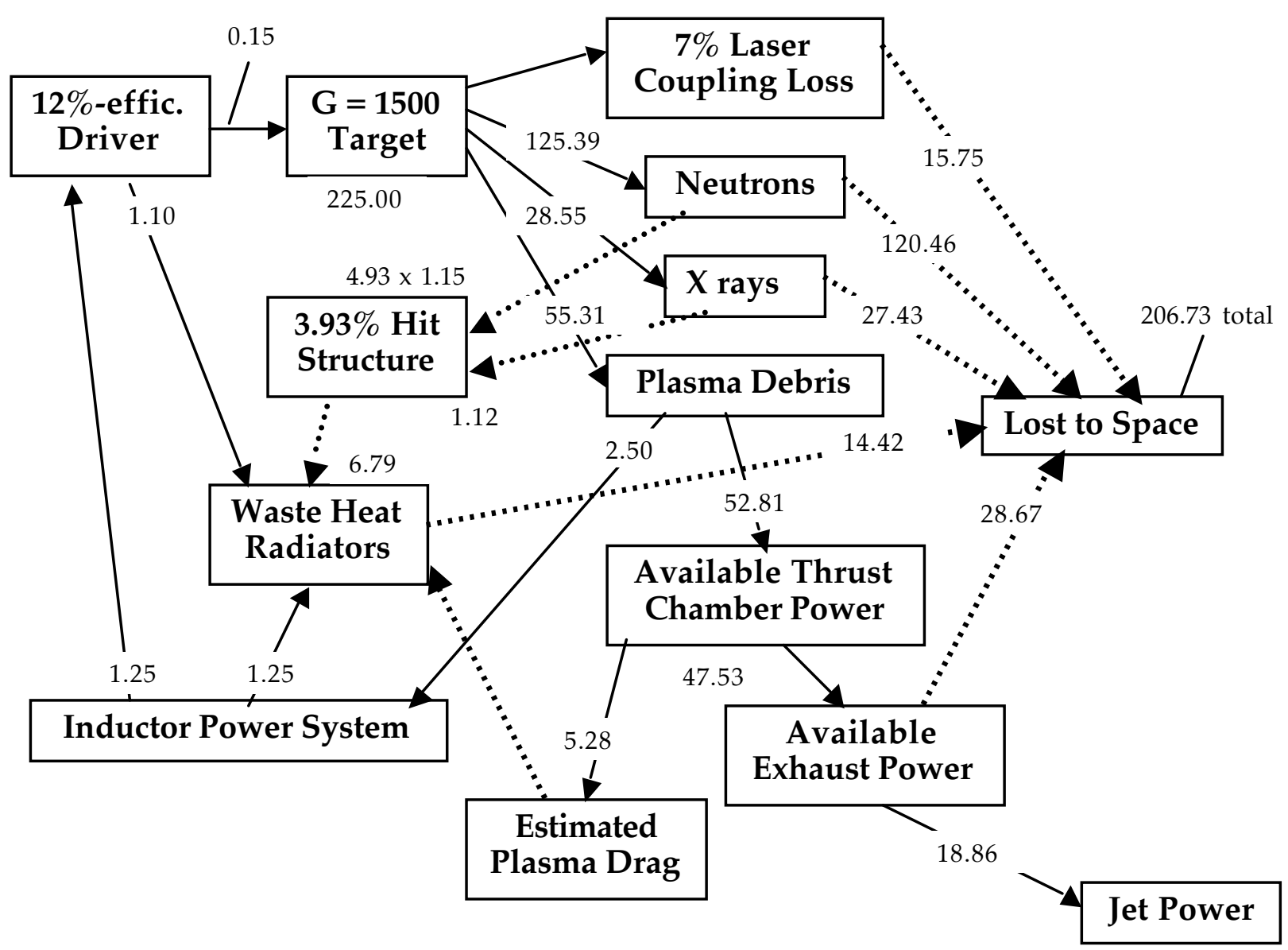

Obviously, one effective way to increase the performance of VISTA is to improve the efficiencies of the various subsystems. 


\section{VI.3 Inductor Power System}

The inductor system extracts power from the expanding target debris plasma as the magnetic-field lines are shoved ahead of the plasma and compressed and then decompressed in the process of deflecting the plasma rearward for thrust. The aspect of this process that is important here is that the magnetic field lines are moved in space toward and then away from the coil windings. Therefore, if another (inductor) coil loop is placed inside the superconducting coil so that the moving magnetic-field lines cross the inductor coil, an emf is generated according to the change in magnetic flux passing through the inductor loop. The emf would have one sign during compression of the lines, and the opposite sign during decompression of the lines. The emf thus has the same time scale as the debris-magnet interaction ( $\mathrm{a}$ few to $10 \mu \mathrm{s}$ ). The extracted energy due to the induced emf is small $(\leq 5 \%)$ compared with the total energy being converted from plasma kinetic energy to magnetic potential energy and back to kinetic energy, so engine thrust is not significantly affected. This is the essence of the inductor power-conversion system. Several options are then available for the power-conditioning subsystem.

Figure VI-2: Overall Power Flow (in GW) for the Advanced-Technology Mission to Mars Using the Rankine-Cycle Power System

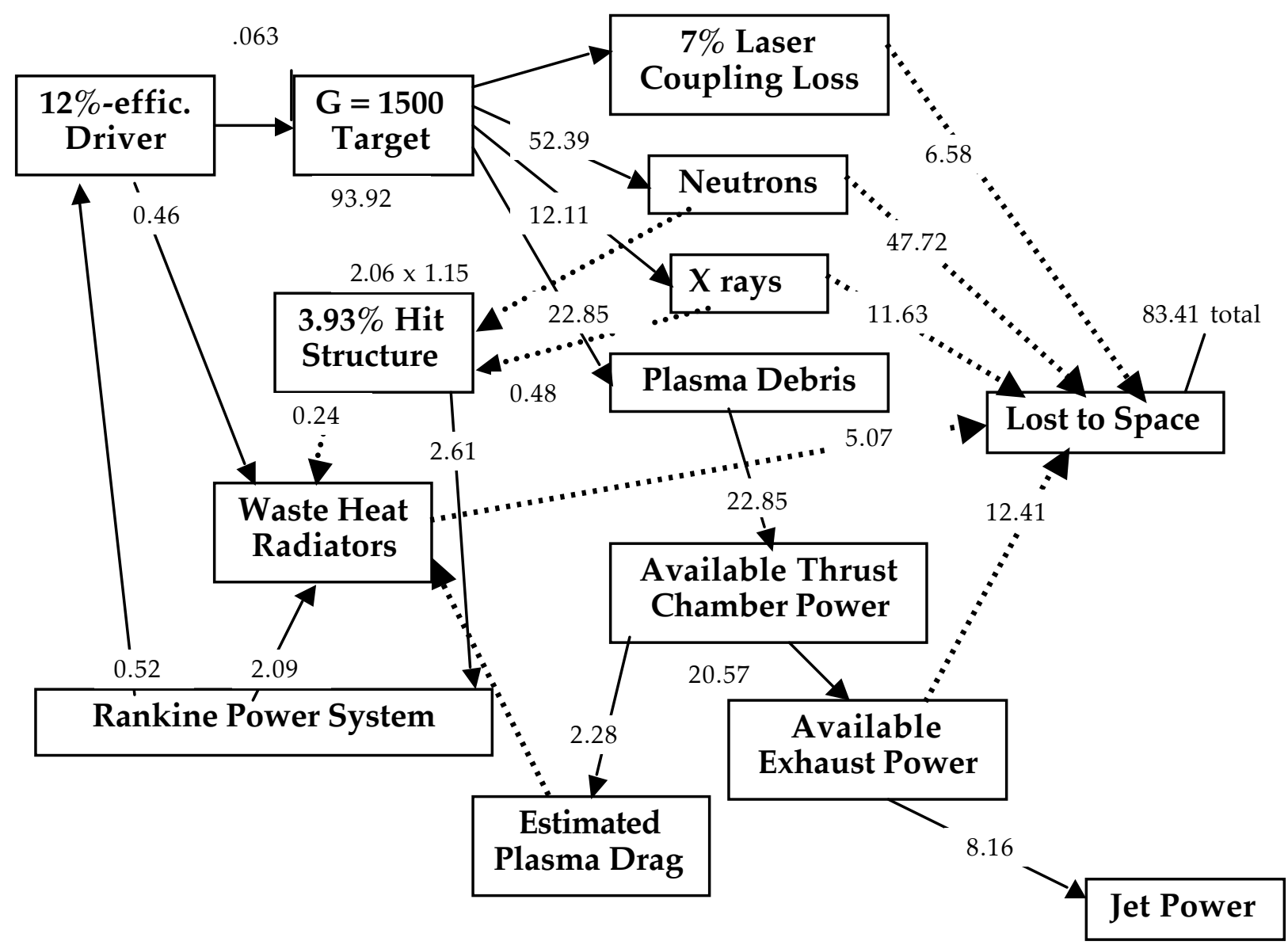




\section{VI.3.1 Inductor Power Conversion Subsystem}

Large forces may develop between the superconducting coil and the inductor coil because the generated emf produces fields which oppose the change in magnetic flux through the inductor loop. It is therefore practical to use just the interior surface of the superconducting magnet coil shield (i.e., the beryllium interior ablation liner) to serve as the inductor loop, instead of incorporating a physically separate inductor loop. It may even be possible to use the lithium liquid in the coil shield as the loop conductor. We leave to a future study just how this system should be optimized.

If the interior surface of the coil shield serves as the inductor loop, it has three functions: 1) to contain the lithium neutron absorber, 2) to act as an inductor coil, and 3) to prevent material vaporization from incident $x$-rays. This material may be electrically divided into the equivalent of multiple windings to form a modular and/or redundant subsystem, if needed. The total mass of this part of the subsystem is insignificant, so various options should be considered.

For a typical pulse, the rise and fall time of the inductor signal is roughly several microseconds. The bulk of the power conversion system lies in expanding this signal to the 1-ms pulse needed for the driver laser diodes, and in delaying the converted pulse for one engine cycle. We did not design this important part of the conversion subsystem, and leave such details to a future study.

As a backup conversion system, we examined the feasibility of a modular system to convert the inductor signals to a dc $600-\mathrm{kV}$ level for use by a KrF-type driver. For $5 \mathrm{MJ}$ on target and a $6 \%$ efficient driver, $83 \mathrm{MJ}$ are required per pulse. As an example, note that this is easily supplied electrically by 260 modules, each of which supplies $320 \mathrm{~kJ}$ through an equivalent circuit that is a series LC of $0.5 \mathrm{mh} \times 4 \mathrm{mf}$ with an opening switch. The switch opens at peak field compression, and must be able to carry $750 \mathrm{kA}$ and resist a back voltage during the field decompression phase. Even though the availability of such switches in the VISTA time frame is highly likely, this subsystem is quite massive because it introduces capacitative storage and is thus undesirable.

\section{VI.3.2 Inductor Power Conditioning Subsystem}

Because we did not design the inductor power system in detail, we cannot give precise masses for the subsystems. We feel, however, that the baseline inductor system will have a mass that is insignificant compared with other spacecraft systems, unless the backup capacitative subsystem is incorporated. In the latter case, the same type of system employing resonant capacitative discharge or the discharge of charged transmission lines can be used here as for the Rankine power-conditioning subsystem.

\section{VI.4 Rankine Power System}

The backup power system consists of a 20\%-efficient Rankine power cycle utilizing the neutron deposition in the superconducting magnet coil shield and a companion lithium blanket as a heat source. The Rankine system feeds a power conditioning system employing a high-frequency turbo-alternator with capacitative storage. The relatively low efficiency for the Rankine system is based on minimum-mass rather than maximumefficiency considerations. We designed the entire system based on a KrF-type driver, as 
originally envisioned, although a DPSSL was chosen in the final selection process for rather obvious reasons. Driver pulses can hence be formed through either resonant capacitor discharge, or by the discharge of charged transmission lines.

\section{VI.4.1 Rankine Power Conversion Subsystem}

The Rankine power cycle utilizes saturated potassium vapor as the working fluid, in a design similar to one presented for a fusion-based power study. ${ }^{77}$ Figure VI-3 is a flow sheet for this power cycle. Fusion neutron energy is deposited as heat in a 1.5-m thick shield of liquid lithium encased in a refractory metal sheet (coated with beryllium) and located in front of the superconducting coil. The lithium is pumped out of the shield and through a counterflow tube and shell boiler, transferring the heat to boiling potassium inside the tubes. Potassium vapor exits the boiler and flows to the main turbine inlet, with a side stream flowing to an auxiliary turbine that drives the centrifugal feed pump. Fluid entering the boiler is preheated by one or more shell and tube regenerative feed heaters. In Fig. VI3 , only one feed heater is shown. Additional heaters utilize turbine extraction vapor. The temperature of the potassium leaving the boiler was selected as $1390 \mathrm{~K}(2500 \mathrm{R})$; this is compatible with components made of Astar-811C and Columbium-1 Zirconium.

Figure VI-3: Power Flow Diagram for the Backup Rankine-Cycle Thermal System

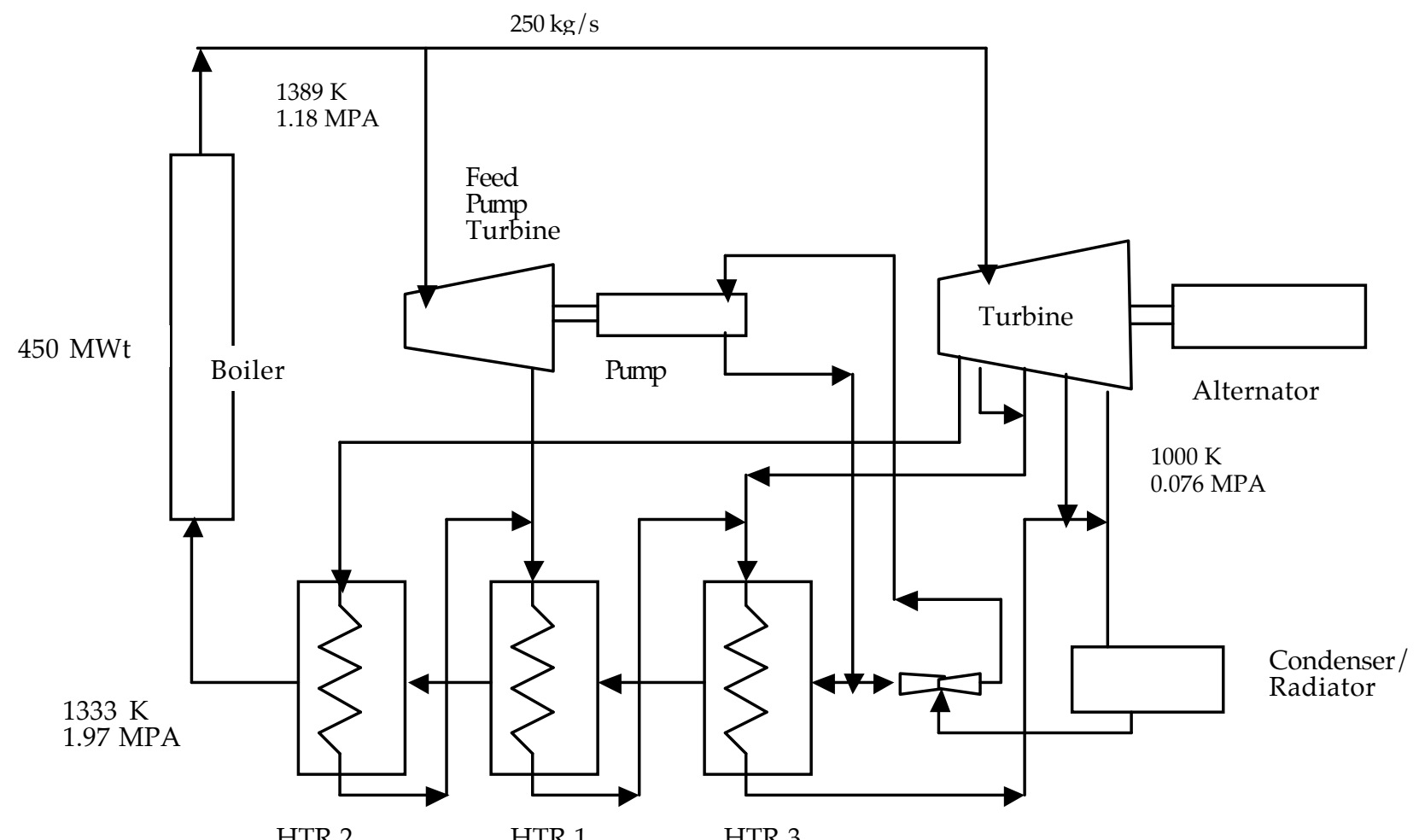

HTR 2

HTR 1

HTR 3

The mass of the Rankine power system was estimated as $1.55 \mathrm{P}^{0.95}$ tons for $\mathrm{P}$ equal to the auxiliary power plus the input power to the driver. This formula was derived by using the computer program ALKACYCL ${ }^{78}$ to determine cycle efficiency for various values of radiator temperature, and varying the temperature at the turbine exit while calculating the mass of each component in the conversion cycle to find the temperature corresponding to 
the minimum total mass. The program also outputted the energy balance, line sizes, and masses for the piping and feed heaters. We determined the masses of the major components separately, using this program. Individual components, and the scaling laws used for each, are discussed in the following paragraphs. For the case shown in Fig. VI-2, the total mass was 569 tons in equipment plus 140 tons in radiators (709 metric tons total); this is much more than the 115 metric tons required for the inductor power system.

We included the possibility of adding a lithium blanket enclosed by $\mathrm{Nb}-1-\mathrm{Zr}$ walls (coated on the interior side with beryllium) for the case in which the $3.93 \%$ solid angle occupied by the coil shield proved insufficient in absorbing enough power from target neutrons and gamma rays to operate the driver. The thickness of all walls was to be estimated for a $99 \%$ survivability in the interplanetary micrometeoroid environment for a mission of at least 80 days. We found, however, that only 3.5\% of the solid angle was necessary for power absorption with the Rankine system, so no additional blanket was required. Nevertheless, we continued to design the Rankine system as if a blanket were to be incorporated.

We sized the lithium piping of the primary heat transport loop to provide steady-state heat removal from the lithium blanket. For this purpose, we assumed a 200-C temperature drop through the boiler and a flow velocity of $6.1 \mathrm{~m} / \mathrm{s}$. Like the lithium shield, the piping wall thickness was selected for protection from micrometeoroid damage.

An EM pump circulates the lithium through the boiler. The pump mass is a function of both the volumetric flow rate and the pressure drop through the lithium piping. The flow rate increases directly with system thermal power. For a fixed length of piping, the pressure drop decreases slightly as the flow rate increases, due to increased pipe size. As a result, pump mass scales very closely with system thermal power.

The turbine is an axial flow type with nine stages and two interstage moisture separators. The stage efficiency is equal to the dry stage efficiency $(0.85)$ degraded by stage moisture. Turbine size depends largely on the total volumetric flow through the turbine. Based on a previous study ${ }^{79}$ a specific mass of $70 \mathrm{~kg} /\left(\mathrm{m}^{3} / \mathrm{s}\right)$ of turbine outlet flow was calculated for a turbine of $100 \mathrm{~kW}_{\mathrm{e}}$ output. The scaling of turbine size with power is difficult to determine, because potassium vapor turbines for megawatt size have not been built. As the turbine size increases, its efficiency increases, suggesting a scaling exponent less than unity. However, the wall thickness must also increase as power goes up due to the large component size. We determined a scaling exponent of 0.9 by comparing the turbine masses for $350 \mathrm{~kW}_{\mathrm{e}}$ and $10 \mathrm{MW}_{\mathrm{e}}$ potassium Rankine power cycle designs having approximately the same temperature conditions. ${ }^{80}$

We assumed that the generator mass scales like a transformer mass, namely to the 0.75 power. ${ }^{81}$ At $5 \mathrm{MW}_{\mathrm{e}}$ output, we assumed that the generator has a specific mass of 0.1 $\mathrm{kg} / \mathrm{kW}$. The number of boiler tubes, and thus the total tube mass, is directly proportional to the thermal power absorbed by the shield. The boiler shell diameter and shell thickness both increase with the square root of the thermal power; thus, the total scaling factor is a direct function of thermal power. We obtained a boiler specific mass of $0.025 \mathrm{~kg} / \mathrm{kWt}$ based on an overall heat transfer coefficient of $8500 \mathrm{~W} / \mathrm{m}^{2} \mathrm{~K}^{82}$ a tube-to-shell temperature difference of $111 \mathrm{C}$, and a factor of 1.5 to account for shell mass. We obtained secondary loop sizes and feed-heater masses from the computer program ALKACYCL. The secondary piping also has micrometeoroid-protective shielding. The heat pipe radiator considered for these calculations, however, is not shielded; we sized the wall thickness to 
accommodate internal pressure only. We assumed that excess heat pipes are installed at the beginning so that $10 \%$ can be lost to micrometeoroid damage during operation.

We assumed here that the radiator was an array of heat pipes of $\mathrm{Nb}-1-\mathrm{Zr}$ construction using sodium as a working fluid. Potassium vapor leaving the turbine condenses as it comes in contact with the evaporating sections of several heat pipes embedded in the turbine outlet piping. The piping tapers as the potassium condenses and subcools before entering the feed pump. We assumed that the radiating temperature is $100 \mathrm{~K}$ less than the turbine outlet temperature. The radiator design has an assumed specific mass of 0.13 $\mathrm{kg} / \mathrm{kW}$ of reject heat at $900 \mathrm{~K}$, and scales with radiator temperature to the fourth power. More advanced radiator designs are of course possible.

We estimate that a minimum total mass of $450,000 \mathrm{~kg}$ occurs at a radiator temperature of $900 \mathrm{~K}$. Of this mass, $14,000 \mathrm{~kg}$ is due to the alternator. Thus the total Rankine-system mass is $436,000 \mathrm{~kg}$. At $900 \mathrm{~K}$, the lithium blanket is the heaviest component. It increases with temperature because the cycle efficiency decreases, and a greater thermal power is necessary to output the required electric power.

The turbine mass decreases with radiator temperature. As the radiator temperature increases, the mass flow rate increases to provide the same power output at a lower efficiency. However, a higher radiator temperature corresponds to a higher saturated liquid pressure, which has the effect of reducing volumetric flow rate even though the mass flow rate is increasing. The turbine curve turns upward at higher temperatures when the mass flow increase dominates the volumetric flow decrease. The generator mass remains constant as the radiator temperature increases, since electric power output is held constant.

A minimum radiator mass occurs because specific mass decreases with radiator temperature while the amount of heat rejection increases due to the decreasing cycle efficiency. A minimum mass of $130,000 \mathrm{~kg}$ occurs at $1000 \mathrm{~K}$ although the curve is very flat between $950 \mathrm{~K}$ and $1025 \mathrm{~K}$.

The boiler mass increases with radiator temperature as the efficiency decreases. Primary piping and EM pump mass both increase in order to accommodate the larger flow rate due to a higher thermal power. Secondary piping mass decreases because although the flow rates are increasing, the turbine outlet pressure also increases with temperature, keeping the piping sizes small.

The high-frequency turbo-alternator delivers 3-phase ac current at $1500 \mathrm{~Hz}$ and typically $15 \mathrm{kV}$. The 3-phase output allows acceptable low voltage ripple on the dc bus, requires a modest rectifier parts count, gives adequate rectifier utilization, and simplifies alternator and transformer design.

\section{VI.4.2 Rankine Power-Conditioning Subsystem}

The purpose of the power conditioner is to accept alternator output voltage and convert this ac source of power into high-energy short-duration pulses used to drive the fusion driver. In order to minimize system mass and optimize efficiency, the power conditioner uses a single multi-megawatt 3-phase transformer to perform primary ac voltage step up and electrical isolation. The transformer output is processed with a 3-phase solid-state bridge silicon-controlled rectifier/voltage regulator, and an inductor to provide signal filtering and current limiting in the case of driver fault. The processor is assumed to incorporate high-efficiency, low-mass, moderate-to-fast fault interruption. The output of the processor feeds a capacitor-storage network. We selected capacitor storage to obtain a 
system with high specific energy, low specific mass, and rapid rise-time discharge. The driver is powered from the capacitor bank through a pulse-forming network and a load switch. The capacitors can be resonantly discharged into the load, or even better, through the discharge of charged transmission lines. Distribution to other systems would be through conditioners that would provide the correct potential and modulation for each subsystem.

For the case of a $280 \mathrm{MW}_{\mathrm{e}}$ unit, we estimated the combined specific mass of the Power Conditioning/Processing Unit (PPU) to be $0.37 \mathrm{~kg} / \mathrm{kW}_{\mathrm{e}}$ exclusive of radiators. The design of the buffer capacitors is based on both the solid dielectric capacitor technology developed by Maxwell Labs (funded by the Defense Nuclear Agency), and state-of-the-art polypropylene film technology developed by Sandia National Laboratory (G. W. Mauldin, principal investigator). The total specific energy will be between $400 \mathrm{~J} / \mathrm{kg}$ and $4000 \mathrm{~J} / \mathrm{kg}$. The specific volume is between 0.6 and $0.8 \mathrm{~J} / \mathrm{cm}^{3}$, with $2 \mathrm{MV} / \mathrm{cm}$. The plate material is aluminum.

The mass of the capacitor network can vary from 5 to 55 tons at $20 \mathrm{MJ}$, using the above parameters. The total mass of the power conditioning subsystem for typical driver input requirements (6\%-efficient driver with 5-MJ illumination on the fusion target, and hence $83 \mathrm{MJ}$ of driver input-not $20 \mathrm{MJ}$ ) is correspondingly between 100 and 400 tons exclusive of radiators. Total system efficiency is $97.5 \%$. New device developments assumed include improved semi-conductor switches (high-voltage and high-current, and very fast deep-impurity double-doped silicon devices), capacitors (new materials and new cooling techniques with objective of high energy density and low loss for pulsed operation), and high-frequency magnetic materials for transformer size reduction and efficiency improvement. Obviously, without further technological developments, the Rankine powerconditioning subsystem will be very massive.

\section{VI.5 Energy Multiplication Factor}

As the neutrons and gamma rays leave the target/expellant region and interact in the structure of the coil shield and/or thermal blanket, exoergic (energy-producing) nuclear reactions can occur (e.g., in Be and ${ }^{7} \mathrm{Li}$ ). These reactions can typically increase the deposited energy by a factor of 1.14 through immediate neutron and gamma-ray multiplication. Short-lived activation products tend to increase the energy multiplication factor to 1.17 or 1.18 as these products decay (e.g., by beta decay). If materials such as manganese are added to take advantage of additional exoergic $\left(n, n^{\prime}\right)$ reactions, the energy multiplication factor can be increased even further. For VISTA, we assume a conservative value of 1.15. The power flow in VISTA is therefore enhanced by this factor for all energy arising from $\mathrm{f}_{\text {neut }}$ the fractional energy in "neutron" emissions (Sec. III.6). We emphasize that such energy multiplication is not desirable for VISTA, as it is for terrestrial power production, so our approach here with a multiplication factor of 1.15 is very conservative! We assume that future studies will demonstrate how this multiplication factor can be substantially reduced.

\section{VI.6 Auxiliary Power System}

Approximately $1 \mathrm{MW}$ of dc power must be generated to operate auxiliary equipment, such as the following: crew systems and payload housekeeping; Rankine-cycle blanket EM pumps; EM pumps for coil shield and driver coolants; refrigeration systems (compressors 
and pumps) for the superconductor, propellant tanks, and final laser turning mirrors; target fabrication, acceleration, and injection facilities and coolant systems; load controllers; system and reaction (attitude) controllers; and communications. This power can easily be supplied as a negligible addition to the main power-conditioning subsystems. About $10 \%$ of the power can be supplied by the engine startup power system. In either case, essentially no additional mass penalty is incurred by the auxiliary system, because the subsystems required are already in existence.

\section{VI.7 Engine Startup Power System}

A separate power-generation system is needed to supply roughly $83 \mathrm{MJ}$ to fire the first driver pulse, melt the lithium in the coil shield, and supply power for crew needs during coasts periods or when orbiting a planet. If a $100 \mathrm{~kW}$ SP-100-type system is used, it could supply the start-up power in less than 10 minutes. Such a small fission reactor would have an estimated mass (including coolant and all of the associated equipment) of 5 tons. This system could be kept at minimum power output during engine operation, and would then supply a negligible part of the auxiliary power requirements for the spacecraft.

\section{VI.8 Uncertainties in Power Generation and Their Effects}

It is important to understand that, when flight hardware is developed and a flight of VISTA occurs, there will be uncertainties in power generation, systems masses, and system performance that can each affect the roundtrip time for any mission. These uncertainties are many, including particularly the attained performance of various engine and power-system components relative to what VISTA design specifies for performance and system mass (e.g., uncertainties arising from an incomplete understanding of each system, and the mass contingencies considered section XI.3). There are also the variations from specified performance under actual flight conditions due to unforeseen or uncontrollable environmental factors or whatever. Of special concern is the effect of these uncertainties on jet power, radiator performance, and other system factors that can affect performance directly or indirectly through increases in vehicle wet mass. Rather than describe the details for each system mentioned in this report, we have instead made Monte Carlo runs in which EVERY system parameter for VISTA was randomly varied with its own Gaussian distribution having a standard deviation of $10 \%$. We report the effects on trip time in Sections XI.4 for Mars missions and XII.2 for missions to Jupiter and Pluto. The net uncertainty in trip time had a standard deviation that varied from only 7 to $13 \%$ depending on the mission. 
C. Orth, UCRL-LR-110500

\section{THERMAL CONTROL SYSTEMS}

Thermal control systems are required to remove waste heat generated from inefficiencies in the driver and power systems, and from the deposition of target emissions in the coil shield (and blanket, if used). Thermal control (refrigeration) is required to maintain the temperature of the superconductor for the magnet, and the temperature of the propellant tanks.

\section{VII.1 Concepts and Effects of Technology Development}

The most massive thermal-control systems are the radiators that remove waste heat. These systems must radiate about 6,000 MW at peak engine output. With current technology suggesting specific masses near $0.2 \mathrm{~kg} / \mathrm{kW}$ (i.e., 0.2 tons / MW) for heat-pipe radiators operating at $1,000 \mathrm{~K}$ with $14 \mathrm{~kg} / \mathrm{m}^{2}$, the mass of these systems is $6,000 \times 0.2=$ 1,200 tons. Such a mass would severely restrict vehicle performance.

The radiator mass can be reduced by operating at higher temperatures, and using more advanced radiator designs. Because the radiators for the coil shield eliminate most of the radiated power, we have chosen their operating temperature to be $1500 \mathrm{~K}$. This temperature is compatible with a lithium transfer fluid, which is already present in the coil shield. We also anticipate (and assume) that the specific radiator mass at 1,000 K can be lowered to about $0.07 \mathrm{~kg} / \mathrm{kW}$ at $1,000 \mathrm{~K}$. The detailed design of the radiators is left for a future study, but is essentially current technology. The results of these two improvements reduce the total mass of radiators down to a couple hundred tons.

It is important to understand that vehicle performance is highly dependent on radiator technology (i.e. radiator specific mass) even though the fraction of total spacecraft mass in radiators is typically near $10 \%$ for VISTA. The fractional vehicle mass in radiators, $\mathrm{w}_{\text {radiators, }}$ can be approximated for a continuously running engine by the ratio of the radiator mass and the wet mass, the latter being roughly the propellant mass divided by 0.7 (because the propellant mass is roughly 0.7 of the wet mass). Thus,

$\mathrm{w}_{\text {radiators }}=\mathrm{k} \mathrm{E}_{\mathrm{dr}}\left[1.15 \mathrm{G} \Omega \mathrm{f}_{\text {neut }}+\left(1-\varepsilon_{\mathrm{dr}}\right) / \varepsilon_{\mathrm{dr}}\right] /\left\{\left[\mathrm{t} \mathrm{m}_{\text {target }}\right] / 0.7\right\}$

where $\mathrm{k}$ is the specific radiator mass, $\mathrm{E}_{\mathrm{dr}}$ is the driver energy in $\mathrm{MJ}, \mathrm{G}$ is target gain, $\Omega$ is the fractional solid angle subtended by the coil shield, $\mathrm{f}_{\text {neut }}$ is the fractional fusion energy into neutrons, $\varepsilon_{\mathrm{dr}}$ is the driver efficiency, $\mathrm{t}$ is the roundtrip travel time in seconds, $\mathrm{m}_{\mathrm{target}}$ is the total target mass in tons, and we have not explicitly shown the dependence on temperature $\mathrm{T}$. Within any one material range, the radiator mass varies as $\mathrm{T}^{-4}$, but over a wider range than just one material and when increased pipe wall thicknesses are considered for higher temperatures, it varies approximately as $\mathrm{T}^{-2}$. We have assumed the latter dependence, so that we can cover wide ranges in T. In any case, for VISTA parameters and a 140-day mission, Eq. (23) shows that Wradiators is approximately $\mathrm{k} / 4$. Thus, with $0.2 \mathrm{~kg} / \mathrm{kW}$, we could expect about $5 \%$ of the vehicle liftoff mass to be in radiators. The radiator mass would hence seem to be a small fraction of the total vehicle mass.

However, when the trip time is optimized with coast periods so that the propellant consumption is roughly fixed, Eq. (23) does not apply because the radiator mass still varies with repetition rate but the total propellant consumption does not. As a result, $\mathrm{w}_{\text {radiators }}$ 
can increases by a significant factor, but we typically find for VISTA that it is near $10 \%$ for vehicle masses not greatly exceeding 6,000 tons. Thus, even for optimized performance, $\mathrm{w}_{\text {radiators }}$ is relatively small.

Nevertheless, the specific radiator mass is very important because it has a major impact on vehicle performance. In order to minimize trip time for a particular mission, we must minimize spacecraft dry mass (i.e. non-propellant hardware mass). This would not ordinarily seem to be the case, because trip time for a continuously running engine with fixed specific impulse is determined only by the vehicle mass ratio (i.e., the ratio of wet mass to dry mass). Hence, if more mass is added in the form of radiators, it would seem that we could add about 4 times this same mass increase in propellant to maintain the same mass ratio, and the trip time would stay the same. In practice, however, there are limits to the total vehicle mass for timely assembly in orbit. In addition, the specific radiator mass also affects the minimum trip time that is possible when the trajectory is optimized with coast periods. In particular, at $0.07 \mathrm{~kg} / \mathrm{kW}, 145$-day roundtrip Mars missions are possible with VISTA; at $0.2 \mathrm{~kg} / \mathrm{kW}$, they are not.

What is important for vehicle performance is thus whether the radiator mass is large compared to the masses of other spacecraft hardware, such as the driver and coil systems. At $0.07 \mathrm{~kg} / \mathrm{kW}$, the radiator mass is comparable to the driver mass; at $0.2 \mathrm{~kg} / \mathrm{kW}$, the radiator mass completely dominates the dry mass. VISTA performance is thus dependent on the level of technology assumed for the radiator systems, down to a specific radiator mass of about $0.05 \mathrm{~kg} / \mathrm{kW}$. Below this level, the radiator mass is much less than the mass of the driver system, so the driver becomes the primary limitation to improved performance. For further details, see Sec. XI.1.

\section{VII.2 Radiator Design With Micrometeoroid Shields}

As indicated in Sec. IX, we designed the spacecraft as a cone with the target at the apex in order to 1) minimize the impingement of target emissions with structural components, 2) produce a stable mechanical structure, and 3) to afford a mobile vehicle with a stable thrust vector under steerage. Because the conical surface must exist to support the driver and other systems, and to separate the crew from the target explosions, we designed the wasteheat radiators so that they would actually form the conical surface. Appropriate stiffeners were added to increase rigidity. We chose heat-pipe radiators because of their conservative design, although there are many other types of radiators.

We did not design the heat-pipe radiator system in detail, but the basic structural layout of the radiators can be easily conceptualized based essentially on existing heat-pipe technology. We envision a central heat-exchange ring near the magnet coil with the main feed pipes branching out from it. These main feed lines, when strengthened, form the main rigidity components of the cone. Lateral heat pipes emanate from these main feeds throughout the rest of the conical surface.

We envision the lower portion of the cone being devoted to the driver radiators operating at $900 \mathrm{~K}$, and the upper portion being devoted to the coil-shield radiators operating at $1500 \mathrm{~K}$, provided the hotter coil-shield fluid can be efficiently transported to the upper portions of the conical surface. The reason for this layout is that it is slightly more efficient to have the hotter radiators on top when the thermal viewfactors (form factors) are considered. These viewfactors consider that both the inside and the outside of the cone are active radiator surfaces, but the inside is less efficient (and increasing less efficient 
towards the apex) because part of the viewing solid angle of the radiators is not cold space (assuming the sun is not an issue here) but opposing radiating surfaces. The viewfactor that accounts for this change in viewing efficiency must of course be modified for area devoted to systems structure (driver, target-factory structure, etc.) and the occlusions that such equipment produces. The net result is that use of the inside as well as the outside of the cone adds only at most $67 \%$ more radiating area-it does not double the area. Geometrically, the viewfactor for inside surfaces varies from about 0.22 near the coil shield to about 0.88 near the spacecraft forward rim, with an overall average of about 0.67 . The viewfactor for exterior surfaces is about unity. These viewfactor estimates must be modified to include any micrometeoroid shielding (see below) and re-radiation of power absorbed from other radiating surfaces. A complete thermal analysis must await future study, which may indeed reveal that it is better to locate the coil-shield radiators near the coil shield instead of near the cone rim. With the radiated powers required for VISTA, it is simply not a significant issue thermally where the coil-shield radiators are located.

Considerations of the radiator specific mass are as follows. If we begin with SP-100 specifications and use the NASTRAN computer code to design the required cone strength, we get an average specific radiator mass of about $14 \mathrm{~kg} / \mathrm{m}^{2}$. This corresponds to approximately $0.2 \mathrm{~kg} / \mathrm{kW}$ at $1000 \mathrm{~K}$. However, further investigation reveals that we can incorporate most of the ideas of Rod Hyde's heat-pipe design ${ }^{83}$ and thereby reduce the specific mass to about $5 \mathrm{~kg} / \mathrm{m}^{2}$, or $0.07 \mathrm{~kg} / \mathrm{kW}$ at $1000 \mathrm{~K}$. Both the SP-100-based design and the Hyde-based design allow an extra $10 \%$ in area to accommodate damage from micrometeoroids. One critical difference in these designs is that the Hyde design allows the feed-to-heatpipe transfer region to enclose the main feed lines, thereby offering micrometeoroid protection for the main lines without added mass. The differences between the radiator designs for the driver and the coil shield arise primarily from the different temperatures, $900 \mathrm{~K}$ and $1500 \mathrm{~K}$, respectively. If radiators are needed for the Rankine cycle, they would operate at 1,000 K and would be similar in design to the driver radiators.

A very important issue is whether it is adequate for micrometeoroid protection to merely allow a $10 \%$ loss of radiators during one mission. Although this is the standard approach in the field, we assume that it is not adequate at speeds of several tens of $\mathrm{km} / \mathrm{s}$. Small particulates swept up by VISTA traveling at such speeds will create a hole in the first surface encountered, producing a dispersing shower of reduced-energy particulates out the rear of the first surface. Such a shower will generally not produce a hole when it impacts a second surface a short distance away. We have therefore conservatively assumed that, in addition to the $10 \%$ overall loss, the entire radiating surface of VISTA must be covered with one additional surface (a micrometeoroid shield) to effectively stop these so-called micrometeoroids from damaging the heatpipes. We have assumed that this micrometeoroid shield can be very thin, and hence will add only $10 \%$ more mass to the radiators. Unfortunately, such an extra surface, if it is opaque to the heatpipe radiation, can reduce the thermal efficiency of the radiating system by a factor of two (i.e., the effective radiating temperature of the heatpipes would be reduced by the fourth root of two). The radiator system must therefore be designed to minimize this thermal penalty, starting with the Hyde concepts, in order to obtain both the size of VISTA and the mass per kW that we report. We have incorporated the extra $10 \%$ mass penalty, and assumed that technology will permit the radiator design with the shield to total $0.077 \mathrm{~kg} / \mathrm{kW}$ at $1000 \mathrm{~K}$. A future study must assess whether this level of technology is a significant increase relative to 
existing technology when the extra micrometeoroid shield is incorporated in a system based on the Hyde design concepts.

\section{VII.3 Refrigeration Systems}

We did not design the refrigeration systems for the superconducting medium of the magnet coil, because we have not yet evaluated the heat input from radiation and conduction exposure from the conical radiators, and from neutrons scattering off miscellaneous structures. We also do not yet know the operating temperature of this "warm" superconductor, but we expect it to be significantly above $100 \mathrm{~K}$. We have therefore ignored any significant addition to the vehicle mass from refrigeration systems for the superconducting magnet. A future study must assess whether this approach is reasonable.

As indicated in Sec. III.8, the tritium storage tank equilibrium temperature will be near $400 \mathrm{~K}$, whereas the tritium used in the targets must be liquefied $(\sim 22 \mathrm{~K})$. This requires removing $\sim 2.5 \mathrm{MJ} / \mathrm{kg}$ from the stored gas to make targets. At a repetition rate of $30 \mathrm{~Hz}$ and $2.8 \times 10^{-5} \mathrm{~kg}$ of tritium per target, a refrigerator must be employed to remove a maximum of $2.1 \mathrm{~kW}$. Onboard hydrogen and deuterium must also be liquefied, but they will be stored at a temperature much less than $400 \mathrm{~K}$, because they have no internal source of heat. We have assumed that a 50-ton refrigerator will take care of all of these needs. A future study must address the design of these systems in detail.

\section{VII.4 Thermal Systems for the Magnet Coil Shield}

The energy deposited by neutrons and gamma rays in the coil shield must be distributed to the radiator network (if using the inductor power system) or the Rankine power system and the radiator network (if using the Rankine power system). This system, which was not designed in detail, consists of pumps and pipes to transport 1500K lithium to heat exchangers that can interface with the heat-pipe network.

\section{VII.5 Thermal Systems for the Final Laser Mirrors}

The two final beryllium turning mirrors absorb energy from three sources: 1) direct neutron, gamma-ray, and x-ray emissions from the target, 2) infrared radiation from the hot heat-pipe radiators forming the conical surface of the spacecraft, and 3) heat from the reflection of laser light off their beryllium faces, with a non-100\% reflectivity. The x-ray deposition, infrared input, and laser light absorption all energize the front surface of the mirrors, while the neutron and gamma-ray depositions energize the material volumetrically. We leave to a future study just how these mirrors should dispense with this heat input, and thus not melt. We note, however, that beryllium is second to carbon in its ability to avoid melting, because its cohesive energy density $\varepsilon_{\text {coh }}$ (i.e., the energy required per unit mass to heat the material from room temperature up through vaporization) is approximately $44 \mathrm{MJ} / \mathrm{kg}\left(\varepsilon_{\text {coh }}=9\right.$ for $\mathrm{Fe}, 13$ for $\mathrm{Al}, 37$ for $\mathrm{B}, 60$ for $\left.\mathrm{C}\right)$. 


\section{CREW SYSTEMS}

The Mars mission assumes a payload of 100 tons, complete with landing vehicles and other crew systems to be described below, with 6 to 10 astronauts. The total mass of the crew systems could easily be extended to 200 tons without serious impact on mission performance.

\section{VIII.1 Types of Crew Systems}

The crew systems consist of a spacecraft portion, and a portion for experiments and experiment probes deployed during the mission. The spacecraft portion includes (e.g.) the Mission Module (MM) and the Mars Excursion Module (MEM). The MM includes 3 SpaceStation (SS)-type modules consisting of 2 Habitability Modules and a Laboratory / Logistics Module, as modified for the Mars mission. The MEM consists of a descent stage that stays on the Mars surface, and an ascent stage for return of the crew and samples to Mars orbit for rendezvous with the MM.

Existing solid rocket de-orbit motors based on a late-1980's design are used for deorbiting the MEM prior to Mars landing. An entry heat shield is provided for deceleration and protection during entry, after MEM deployment. Propulsive braking and attitude control are employed for the landing. Descent engines are arranged such that one is subsequently reused for ascent, all using liquid oxygen and monomethylhydrazine (LO2/MMH) propellant.

The lander portion of the vehicle includes a pressurized crew module/laboratory, experiments, and exploration provisions. The latter include surface mobility provisions such as a rover vehicle having power, communications, and thermal control capability. Extra Vehicular Activity (EVA) capability is provided from the crew module. Upon completion of the surface mission, the crew and samples return to Mars orbit in the ascent stage, leaving most of the landed mass on the surface. The MM remains in Mars orbit with a crew of 2 persons.

The crew spacecraft concept is based on a partial-gee in-transit environment that provides the simplest configuration approach. Two end-to-end large-diameter modules utilize a floor across their midsections, which would house much of the Environmental Climate Laboratory Safety System (ECLSS) as well as power and other required equipment. The cylindrical walls would be free for experiments, bunks, and other facilities. The EVA airlock rests between the two modules, granting access from both. Crew quarters are housed inside the propellant tanks for radiation shielding.

\section{VIII.2 Crew Safety}

Preliminary calculations show that the total spacecraft systems mass should be sufficient to provide adequate protection for the crew from background cosmic radiation, solar flares, and scattered fusion neutrons if the mass distribution could be effected to be equivalent to a protective shell of water at least 3 to $4 \mathrm{~m}$ thick (see general discussion in Section XVI.5). The best way to accomplish such a distribution is to locate the crew quarters inside the propellant tanks located at the lip of the cone. No detailed layout activity has been performed for this arrangement, but it would require the crew to reduce its 
circumferential access through the tanks as propellant is used during the mission (e.g., $100 \%$ access initially, 50\% access when the propellant is half gone). Equipment distribution would necessitate packaging most of the spacecraft equipment around the walls of the pressure vessels for maximum shielding effectiveness. Packaging of fluids such as the expellant around certain habitable modules may be required, but we did not design the layout in detail. Difficulties associated with such a shielding concept include propellant boil-off routing, tank-mass support arrangement, interfaces, integration, and module visibility to space.

\section{VIII.3 Crew System Masses}

We considered only a conceptual layout of the MEM propulsion subsystems and their masses. These included the aerobrake/ heat shield mass for the MEM. The 2.44-m (80-ft) reusable aerobrake mass for the aerobraking vehicles was estimated including heat tiles of the Orbiter type. We used the N204/MMH (storable) concept as the reference including the descent and ascent stages. We considered 3 engines during descent to the Mars surface, but only one for the ascent phase of the mission. We considered preliminary mass estimates for crew consumables, as scaled from a 2-year to a 150-day mission, and for the Habitability Module \#1, Habitability Module \#2, Laboratory/Logistics Module, the MEM, and the Science Probes. The micrometeoroid shield and outer insulation masses were included with the structures. We estimated an airlock mass for the Lab/Log Module on the same line with the mass for an aerobrake/ heat shield for the MEM. The Habitability Module would supply power and ECLSS to the Lab/Log Module. A 15\% contingency was included on all the dry masses because most of the hardware was new and considered current technology equipment. We included spares for non-structural masses at 3\% / year. Fluids, consumables, and propellants were considered separately for each module. The de-orbit propulsion system included extra propellants for limited plane changes and landing site selection capability. The mission/science masses were only representative and would change as requirements are established. The crew masses included six people with flight suits. Shielding could be provided in the modules, mostly from the equipment and consumables, provided the layout of each module is carefully done with shielding as the driving requirement. If this is done, we estimated the effective thickness of aluminum to be approximately $3.8 \mathrm{~cm}$ for the Habitability Modules and $4.7 \mathrm{~cm}$ for the Lab/Log module, assuming even distribution of equipment throughout each module. The minimum thickness required is estimated to be $4.5 \mathrm{~cm}$, ignoring the cancer risks discussed in Sec. $X$, so a primary challenge for spacecraft designers will be to package equipment sufficiently densely (at least in a "storm shelter" region) and to utilize surrounding propellant storage in such a way that additional shielding mass will not be required.

\section{VIII.3.1 Crew Environment}

There should be little audible indication of engine operation at repetition rates under $20 \mathrm{~Hz}$, except through harmonics. Vibrational hazard should be minimal, because the flexibility of the conical structure is sufficient to damp out impulsive loading within about 1 $\mathrm{m}$ of the coil. Thermal and other environmental requirements are provided by the usual crew systems. 
Artificial gravity of $0.0011 \mathrm{w}^{2} \mathrm{R}$ gee is available at radius $\mathrm{R}$ meters if VISTA is rotated about its axis at $\mathrm{w}$ rpm, so 2 to $3 \mathrm{rpm}$ should provide adequate gravity at the crew quarters inside the propellant tanks (e.g., $3 \mathrm{rpm}$ provides 0.8 gee at $\mathrm{R}=80 \mathrm{~m}$ ). Future studies must ascertain the tradeoff between lower rpm for structural considerations and higher rpm for sufficient crew physiological health. 
C. Orth, UCRL-LR-110500

\section{SPACECRAFT DESIGN}

\section{IX.1 Design Requirements}

Figure I-1 shows the overall spacecraft structure. We chose this design based on a number of design requirements:

1. An orientation of the target firing location in relationship to the plane of the superconducting coil that maximizes jet efficiency.

2. A design that minimizes direct radiation exposure for all systems and the payload.

3. An x-ray ablation loss per fusion detonation that is negligibly small compared with the expellant incorporated in each target.

4. A geometry that would provide for large radiator surfaces for waste-heat removal, placed in a structurally efficient manner.

5. A geometry that minimizes radiation exposure due to scattered neutrons.

6. A coil shield geometry that essentially excludes all neutrons and gamma rays from getting to the magnet superconductor material (matrix).

7. Minimum radiative view factor of cryogenic components (coil, propellant tanks, target launcher) and payload to hot radiative surfaces.

8. A geometry that is steerable in a stable manner.

9. A geometry that provides, in a structurally efficient manner, mounting locations and appropriate fields of view for the magnet coil, coil shield, coil refrigerator and coolant pumps, startup nuclear reactor, inductor-coil system, target accelerator/injector and coolant systems, laser driver and components (capacitor bank, power processors, mirrors), final laser turning mirrors and coolant system, propellant tankage and refrigerator, thermal radiators, payload, target storage and assembly facility, etc.

Many of these requirements, but not all, can be satisfied by starting with the geometry specified by Hyde, ${ }^{84}$ who had already determined the optimum target-coil geometry to maximize jet efficiency with the added constraint of producing some tritium in the lithium of the coil shield. Although the latter constraint is important, it is not necessarily relevant here, but we nevertheless retain Hyde's basic geometry with a 50-degree angle between the axis of symmetry and a line from the target through the coil (see Fig. V-2). The target location could be moved slightly away from the coil plane and increase the jet efficiency, but only very slightly (see Sec. V.6 and the next section below), so we ignore this change.

\section{IX.2 Configuration Layout}

The optimum spacecraft geometry is determined by the size of the magnet coil and the positioning of the target relative to the plane of the magnet coil. The size of the magnet is subject to two constraints: 1) the magnet structure and shield must be far enough away from the target so that material is not ablated each pulse by target emissions; and 2) the magnet must not be so far away that the expanding target debris plasma cools excessively to allow significant recombination (and hence magnetic-field penetration) until after being deflected by the magnet. The former constraint requires the coil shield to be roughly $15 \mathrm{~m}$ 
from the target, assuming use of the most resistant materials (e.g., Be, C, $\mathrm{B}_{4} \mathrm{C}$ ); the latter constraint is not well understood at this time.

The positioning of the target relative to the plane of the coil must be accomplished to maximize jet efficiency. Hyde ${ }^{85}$ did this for a 6.5 -m-radius coil using a computer program that calculated the interaction of the debris with the magnetic field, including the hydrodynamics of the debris. (He then moved the target slightly closer to the coil plane to enhance tritium production in the lithium coil shield, but this is not of great significance here.) We therefore started with Hyde's general layout, increased in scale by a factor of two to get the shield at least $15 \mathrm{~m}$ from the target. This layout has a 50-degree half-angle between the axis of the magnet coil and the cone subtended by the centerline of the coil at the target (see Fig. V-2). We shall assume that this scaled-Hyde configuration is not too large for the constraint based on recombination cooling of the plasma. A future study must prove whether this assumption is valid.

The optimum spacecraft geometry based on this layout is determined by the neutron/gamma-ray shield for the magnet's superconductor (the coil shield). This shield must be located on the interior side of the coil, facing the target, to shield the magnet superconductor from neutron and gamma-ray depositions. Otherwise, the resultant heat input to the superconductor could cause it to transition to a normal state, causing shutdown of the engine. The coil shield is sized in angular extent (as seen at the target) for minimal neutron impingement consistent with neutron scattering in the shield itself. That is, the shield is sized first by line-of-sight considerations from the target to the angular limits set by the coil windings and associated hardware. The shield is then enlarged slightly to protect the superconductor from neutrons that might scatter in the edges of a smaller shield and still penetrate the superconductor. The shield design that satisfies these constraints fills $3.93 \%$ of the solid angle seen by the target (see Fig. V-2).

This shield casts a conical shadow that is free of all direct target emissions, because material thick enough to stop neutrons and gamma rays will certainly stop the $\mathrm{x}$-rays (and any stray debris). All spacecraft systems, including laser drivers, power processors, target injectors, etc., can thus be located within this shadow and thereby be protected from target emissions. In fact, it is this shadow concept that determines the conical shape of VISTA, and differentiates our design from the pencil-like design of Hyde, which has less steerage stability. The conical design minimizes the fraction of fusion neutrons that are intercepted by the spacecraft, thus minimizing both the amount of heavy shielding and the amount of spacecraft heating (and therefore the resultant mass in waste-heat radiators). Some components mounted in the shield's shadow may still require local shielding from neutrons scattered from the coil shield or secondarily from the radiator surfaces.

The conical surface is the primary load-bearing structure, onto which must be attached the driver systems, target injector, power processors, et cetera. Local stiffening is provided as needed in the shell to support mounted components and provide structural attachment points. The conical surface is composed of many heat-pipe radiators integrated together and spaced between the longitudinal load members of the cone. The cone thus serves a dual function, and no additional surfaces are required to radiate waste heat from the driver, coil, laser mirrors, and power systems.

The torroidal propellant tank, which is mounted on the base of the cone to minimize its field of view to the hot radiator surfaces, also serves dually as a stiffening ring for the conical structure. The payload is mounted topside (or even perhaps inside) of the propellant 
tanks, minimizing its radiation exposure, minimizing its thermal loading from the radiators, and providing maximum structural isolation from the pulsed fusion drive.

Figure I-1 shows the overall spacecraft structure. The detonation site for the fusion microexplosions is located at its apex, and the entire vehicle travels with the blunt end of the cone facing forward. The torroidal magnetic thrust coil $26 \mathrm{~m}$ in diameter is located $10.9 \mathrm{~m}$ forward of the detonation site, and its magnetic field deflects aftward the charged debris from the fusion microexplosions.

The conical shell is beneficial for additional reasons. Such a vehicle has favorable inertial properties to be spun about its centerline axis (the drive axis) to provide artificial gravity for the crew. The cone might also serve as a substrate for a very large electronically steerable phased array communication antenna able to point its beam in any desired direction. The open cone could also serve as a hydrogen scoop to gather supplementary expellant mass on an interstellar mission.

\section{IX.3 Final Turning Mirror Structure}

The two 12-m-diameter final laser turning mirrors are mounted off the conical shell on two 30-m-long support trusses so as to be opposite each other, each $40 \mathrm{~m}$ from the detonation site. These mirrors and their pointing systems will be similar to those developed for the SDIO Beam Control Experiment spacecraft. ${ }^{86}$ These two mirror systems, their support structure, and the outlets of the target injectors will be the only spacecraft components outside the drive coil shield's shadow and exposed to direct neutron radiation. This radiation exposure presents major challenges for their design, and they will require active cooling systems to support them (Sec. VII.5).

\section{IX.4 Integration of Conical Shell and Radiator Structure}

Section VII.2 describes the design of the radiator subsystems. A NASTRAN computer run for the spacecraft structure composed of heat-pipe radiators having mass area densities near $10 \mathrm{~kg} / \mathrm{m}^{2}$ indicated that stresses will be under $3 \mathrm{kpsi}$. Radiators with mass area densities as low as $1.5 \mathrm{~kg} / \mathrm{m}^{2}$ might still be acceptable for idealized stresses, but one must be concerned about stiffening for local buckling, structural stiffness (first mode frequency) for stability and control, and manufacturability, among other things. Clearly much more work is needed to understand the subtleties of the integrated conical shell/radiator structure.

\section{IX.5 Assembly in Orbit}

The fully assembled spacecraft does not have the configuration or the structural integrity for an earth-surface launch. We assume that the ship would be assembled from sections launched into low earth orbit (LEO). After assembly, the ship would be translated into an orbit above the radiation belts (e.g., a 700-km nuclear-safe orbit). At this higher orbit, fueling and final checkout would be performed by remote handling techniques. During these procedures, only the small fission reactor for auxiliary power would be activated. Only after final checkout would the ship be piloted. If the launch is made from a lunar orbit, manning would proceed after insertion into and checkout in the lunar orbit. 
C. Orth, UCRL-LR-110500

\section{ENVIRONMENTAL HAZARDS}

\section{X.1 Tritium Hazards}

\section{X.1.1 Tritium Hazards for Fuel Transport Up Through the Atmosphere}

\section{X.1.1.1 General Considerations}

Tritium is a low energy $\beta^{-}$emitter, having a maximum energy of $18 \mathrm{keV}$ and an average energy of $5.5 \mathrm{keV} .{ }^{87}$ As such, tritium is not a serious radiological hazard unless it is taken inside the body, because low energy $\beta^{-}$emissions can be harmlessly stopped by clothing or the outer layers of skin. However, once inside the body, tritium can cause serious or even fatal radiation damage if present in sufficient quantities.

One method of bodily entry for tritium is respiration. A passing gas cloud containing tritium (diatomic $\mathrm{T}_{2}$ ) can enter the lungs. Once there it can either directly attack the lungs or can be absorbed into the blood stream and spread throughout the body. At this point it may attack the body as diatomic tritium or may be converted into tritiated water as a result of metabolic processes or molecular conversion.

The primary danger of tritium lies in its chemical similarity to normal hydrogen $(\mathrm{H})$. One of the results of this chemical similarity is molecular exchange. ${ }^{88}$ This is simply a process by which a hydrogen atom and a tritium atom swap places when placed in contact. (e.g., $\mathrm{H}_{2} \mathrm{O}+\mathrm{T}_{2} \rightarrow \mathrm{HTO}+\mathrm{HT}$ ) This can happen in any substance containing hydrogen that is in the path of the tritium cloud. This represents a danger to plants, animals, and man if significant portions of their environment or even their own bodies become radioactive.

While not yet proven, it is believed that some molecular exchange may be the result of a photochemical process in which $\mathrm{H}$ atoms are liberated by incident photons. ${ }^{89} \mathrm{~A}$ subsequent recombination with tritium rather than ordinary hydrogen results in a "molecular exchange." This process would be important in plants and on tissues exposed to sunlight during the tritium release. Isotopic exchange, where adjacent atoms trade places without any exterior influence, is also a probable contributor to molecular exchange. ${ }^{90}$ Isotopic exchange effects are not normally considered as one hydrogen atom simply takes the place of another. However, if tritium becomes involved in the exchange, HTO will be produced.

Tritiated water can also be formed by combustion. In the lift booster explosion scenario, combustion would be the primary process for the formation of tritiated water. It is estimated that such an explosion within the atmosphere, would result in the complete conversion of all the carried tritium fuel into tritiated water. This is probably a good (if not totally accurate) assumption and is certainly a worst case because tritiated water is a far greater threat to the environment than is tritium itself.

Once in the water form, tritium begins to permeate the environment. Further inhalation occurs from $\mathrm{HTO}$ or $\mathrm{T}_{2} \mathrm{O}$ vapor suspended in the air. Respiration and soil processes equilibrate the internal tritium concentration of plants (feed grass, vegetables, etc.) with that of the tritium cloud in a matter of hours. Milk and beef produced by cattle eating contaminated grass reach high levels of concentration within a few days.

The problem of airborne tritium remains even after the passage of the main plume. This is due to re-emission from the soil and transpiration of water vapor into the air from the leaves of plants in the original contamination area. ${ }^{91}$ This effectively increases the 
duration of the total exposure to humans beyond the original release. This "groundshine" re-emission is not as intense as the first wave because the soil and plants do not reequilibrate with the non-contaminated air as quickly as they were contaminated by the tritium cloud. Part of the reason for this may be the organic binding of tritium within the plant due to metabolic processes and molecular exchange: the tritium that entered freely from the air is now bound to the structure of the plant and cannot return to the atmosphere.

While tritiated water permeates and expands throughout the environment quickly, this association with water gives tritium the following positive qualities over other radioactive contaminants. First, tritium does not seem to seek out and concentrate in specific positions of the body. Since water is present throughout the body, tritium spreads more or less uniformly (as opposed to iodine that seeks out the thyroid, for example). While different organs may have a higher susceptibility to radiation damage, they should all be exposed to the same level of irradiation.

Second, the half-life of water in the human body is roughly ten days. ${ }^{92}$ It would be expected that within ten days of removal from the contaminated area, the tritium inventory in a person's body would be half what it was before removal. The possibility of reducing this half-life exists if measures are undertaken to increase fluid transfer through the body, such as increasing non-contaminated fluid intake and sweating. However, tritium that entered the body as water, may become incorporated into organic structures. Organically bound tritium within the human body would appear to be broken up into two more halflife periods of roughly one month and one year. ${ }^{93}$ The reasons for these staggered "organic" half-lives are presently unknown and more investigation will be required.

Studies have suggested that skin contact with a tritium contamination is sufficient to begin organic bonding. ${ }^{94}$ However, organic bonding resulting from the intake of tritiated water appears to occur more slowly and becomes significant only after prolonged exposure. Washing the skin with alcohol has proven to be an effective decontamination method. Such action must be undertaken soon after exposure, however, because its effectiveness decreases rapidly with time. It would appear that organic bonding of tritium can largely be avoided by timely removal from the contaminated area and subsequent decontamination.

\section{X.1.1.2 Accident Scenarios}

Transferring tritium fuel to orbit will present several possibilities for its entry into the atmosphere. The worst case would be the explosive destruction of the booster due to accident or down-range detonation. Both cases would result in release of the entire tritium inventory and at least a partial conversion into tritiated water (an explosive release in the thin upper atmosphere may disperse the tritium below combustion concentration before it can be completely converted). It will be assumed that the booster used will either be the space shuttle or some proposed heavy lift launch vehicle (which is based on the existing parameters of the shuttle's external fuel tank). In either case the hydrogen/oxygen fuel on board would detonate as roughly 1.5 million pounds of TNT (700 metric tons).$^{95}$

An upper atmosphere explosion (above the stratosphere) would most likely present the least environmental danger. The thin atmosphere will typically allow only $50-75 \%$ of the booster's fuel (assumed hydrogen/oxygen) to be consumed in the explosion, with the rest being dispersed below combustion concentration. In this case it would be possible for a portion of the tritium to remain in an elemental state, and escape the Earth's gravitation. Any tritium compounds too heavy to escape should remain in the upper atmosphere for up to five years and could be accounted for in a world-wide background dose. 
There is a possibility that the $\beta^{-}$emitted by upper atmospheric tritium could cause damage to orbiting satellite solar power systems. While their energy is too low to directly damage satellite components, it is possible that they could prompt the creation of colorcenters in the fused quartz that is used as a protective covering for the solar arrays. These color centers may reduce the power available to a satellite enough to make it unusable. At geosynchronous orbit $(30,000 \mathrm{~km})$, the maximum electron trapping flux of the Earth's magnetic field is on the order of $10^{15} \beta^{-} / \mathrm{cm}^{2} /$ day. It is estimated ${ }^{96}$ that 3 tons of tritium will produce a $\beta$ flux of $3 \times 10^{13} \beta^{-} / \mathrm{cm}^{2} /$ day within one day of being released, and therefore should not exceed the trapping flux maximum. The $\beta^{-}$released could remain within the magnetic field for up to a year before becoming de-trapped. This converts to a dose to the satellite surface of $10^{-6} \mathrm{rad} / \beta^{-} /$day, with a total dose of close to $10 \mathrm{Mrad} /$ day. In general, fused quartz coverings are designed to absorb $10 \mathrm{Mrad}$ over the lifetime of the satellite, and the slight discoloration is not normally a constraint on the lifetime of the satellite. Further studies are needed to determine exactly how much discoloration can be tolerated before power drain exceeds power produced. However, at a lifetime dose per day, this could be a significant problem.

A stratospheric explosion would likely result in complete conversion of elemental tritium into tritiated water. The stratosphere's stability would prevent any substantial amount of plume rise as a result of the explosion; therefore, the tritium can be assumed to have been released at the altitude of the explosion. Stratospheric water vapor spends about two years in the stratosphere before entering the troposphere on a world wide average. This average will change with a given latitude: longer for equatorial and polar regions and shorter for the mid-latitudes. An explosion in the stratosphere would most likely be accounted for in a world-wide dose as horizontal transport and mixing is much faster than vertical transport and mixing in the stratosphere. ${ }^{97}$

The worst case is an explosion in the troposphere, not only because of its closeness to the Earth's surface, but also because of the instability of the troposphere. This instability will allow a substantial plume rise following an explosion. This plume rise will have the effect of lessening the dose received near the explosion site (relative to the dose without plume rise) but will increase the area over which the contamination is spread. Upon detonation of the booster's liquid fuel tank, a plume would be created with a 250-m radius at detonation level. By the time the plume is stabilized, it would be $750 \mathrm{~m}$ in radius, centered $2500 \mathrm{~m}$ above its initial position. ${ }^{98}$ These values will hold for virtually any altitude in the troposphere except near the tropopause. The stability of the stratosphere will impede entry of any gases from the troposphere and will most likely reflect them back down.

It is estimated that the recycle time of water vapor in the troposphere is between 1 and 2 weeks. As a tropospheric explosion would most probably result in complete conversion, it could be assumed that the tritiated water will have been deposited on the surface within two weeks (ignoring "groundshine" re-emission). Exceptions to this may result if the release is near the jet stream, because releases of $\mathrm{Pu}^{238}$ near the jet stream have resulted in such quick global mixing that they were absorbed into the world-wide background. ${ }^{99}$

We used the HOT SPOT computer program, ${ }^{100}$ developed by Steve Homann at LLNL, to simulate an explosive tritium release from the launch pad. The program includes factors of skin absorption and inhalation (excluding those from re-emissive groundshine) and does not include any factors for ingestion. This makes the program an approximation only for areas in which local food and water are not consumed. Both of these are assumed to come from outside the affected area. It is assumed that 3 metric tons $\left(3 \times 10^{10} \mathrm{Ci}\right)$ of tritium are released under moderately stable (class F) weather conditions, with a ground 
wind speed of $3 \mathrm{~m} / \mathrm{s}$. These parameters constitute a worst-case release of the entire tritium inventory into a relatively non-dispersive environment. The program found doses out to $300 \mathrm{~km}$ from the explosion site and computed a fifty year dose commitment (CEDE) for a $100 \%$ tritiated water release. Dose commitment is the summation of the initial dose and the doses due to residual contamination. We also considered a non-explosive case that assumed a 20 meter initial release height, which models a containment breach without fire or explosion and a release of $100 \%$ elemental tritium. (The HOT SPOT program normally produces results out to $100 \mathrm{~km}$. Steve Homann modified his copy of the program to extend the distance to $300 \mathrm{~km}$, even though the Gaussian plume dispersion model becomes less reliable with distance. Calculations near $300 \mathrm{~km}$ are therefore more illustrative than accurate, but the program modification did not effect the accuracy of the program at distances less than $100 \mathrm{~km}$, and the graphics capabilities were left unmodified.)

In the explosive case, the maximum dose was 910 rem at a range of $0.1 \mathrm{~km}$ and dropped to $21 \mathrm{rem}$ at the $300 \mathrm{~km}$ limit of the program. The non-explosive release provided a maximum dose of $0.617 \mathrm{rem}$ at $1.16 \mathrm{~km}$ and dropped to $2.5 \mathrm{mrem}$ at $300 \mathrm{~km}$. The HOT SPOT runs for both the explosive and non-explosive cases provide interim data and include CEDE deposition plots and contour profiles.

Absorption of tritiated water through skin absorption and inhalation is $10^{4}$ times that for elemental tritium. In the event of a non-combustive release, atmospheric tritium has been shown to oxidize relatively slowly, about $1.5 \%$ / hr. ${ }^{101}$ However, if this tritium should reach the ground, oxidation occurs very quickly, with complete conversion in only a few hours. This makes soil conversion the dominant non-combustive oxidation method, with atmospheric conversion becoming negligible by comparison.

\section{X.1.1.3 Possible Safe Transport Scenarios}

Evacuation-level dose limits must be considered for any transport scenario. Current limits make an evacuation plan a consideration if public dose levels could fall between 1 and 5 rem. Dose levels over 5 rem require evacuation. ${ }^{102}$ The cost and political implications of requiring local citizens to acknowledge an evacuation plan is something that needs to be considered if releases producing greater that 5 rem are possible.

Three possible safe transport scenarios seem feasible: tritium production in space, launching from a remote site, and division of the tritium between several launches. Tritium production in space would completely alleviate Earth-bound tritium considerations and would be the least environmentally hazardous. It would require the construction and manning of a facility that could manufacture and store the tritium, or require onboard production of tritium (Sec. III.9). In the case of a separate facility in space, it is assumed that tritium would be made from neutron bombardment of lithium. The facility would need to contain a neutron source and would need to have a dependable supply of lithium. While this is the safest method, it would be costly, both in time and expense. The size of the facility required to produce a sufficient amount of tritium by the launch date will need to be considered. Terrestrial production of tritium may take place at many locations and the fuel brought together at the launch site. Space production will be restricted to a single facility (assuming redundant facilities would not be economical) and subject to any shutdowns that might occur.

Launching from a remote site, while moving the danger away from population concentrations, does not reduce the hazard to the local environment and will very likely meet with severe political opposition. Considering the nation's current difficulties of finding mutually agreeable burial sites for even low-level nuclear wastes, it is not likely that anyone will passively accept the hazard level that the release of any amount of tritium might produce. 
The most promising approach may be the distributed launching of the tritium load. Any given accident would not release the entire tritium inventory and the case in which every launch would involve such an accident is unlikely. Many launches will be needed in any case to transport spacecraft components to the orbital construction site. Depending on the number of launches required for normal construction, it may be feasible for a small amount of tritium to be carried on each of these flights. However, this would require a large number of launches.

Assuming a launch site boundary of $5 \mathrm{~km}$ (the worst case for Cape Canaveral), the HOT SPOT program was run again to determine how much tritium could be on board and still meet the EPA evacuation restrictions mentioned above. A dose of 1 rem was received at $5 \mathrm{~km}$ for an explosive release of $5.5 \mathrm{~kg}\left(5.5 \times 10^{7}\right.$ curies $)$ of tritium. If 3 tons of tritium were needed in orbit (which is somewhat more than is needed for DT fuel), this would require 546 launches. This prohibitively large number of launches could be lessened by increasing the allowable dose regulations or increasing the distance between launch sites and the site boundary. For example, to decrease the number of launches to $400,7.5 \mathrm{~kg}$ of tritium could be carried per launch producing an explosive accident dose of $1 \mathrm{rem}$ at $10 \mathrm{~km}$. To decrease the number of launches to $112,27 \mathrm{~kg}$ of tritium could be carried per launch, producing an explosive accident dose of $5 \mathrm{rem}$ at $5 \mathrm{~km}$. On the other hand, if only $2 \mathrm{~kg}$ of tritium is needed, as for DD fuel with DT hotspots (Sec. XV.1), then only a single launch is required to meet EPA evacuation restrictions.

In summary, onboard generation of tritium as explained in Sec. III.9 may be the most environmentally preferable scheme, especially for outer solar system missions. Nevertheless, if DD fuel is used with DT hotspots so the tritium inventory drops to several kilograms (Sec. XV.1), then only a single transport through the atmosphere is required. Thus, for DD fuel and the precautions mentioned above, the tritium risks become manageable for either case: transport through the atmosphere or onboard generation of tritium. The makes the use of DD fuel more advantageous.

\section{X.1.2 Tritium Hazards for Onboard Crew Members}

From the previous section, it is obvious that there is no hazard to crew members while they are in their space suits, because the emitted beta ray cannot penetrate even one layer of thin clothing. However, systems must be included to prevent any tritium from getting into the food or water ingested, or from getting into the gases breathed by the crew members. In either case, the tritium could end up in a water state inside the human body, and could thereby cause serious consequences. Nevertheless, a modification of procedures used at terrestrial laboratories (e.g., LLNL) should suffice for adequate crew protection.

\section{X.2 Neutron Activation of Spacecraft Components}

There are two types of neutron activation of spacecraft components. One is the direct activation of components (such as the coil shield itself and the final turning mirrors) that are situated near the target and not shielded by the superconducting-magnet coil shield. The other is the minimal activation of almost all components resulting from neutrons scattering from the coil shield so as to be redirected to strike components that would otherwise be shielded.

The direct activation of components near the target could be significant, especially if refurbishment of these components becomes necessary. The treatment of this activation and its effects on refurbishing operations must await future investigation. On the other 
hand, the activation resulting from scattered neutrons is not considered to be significant, but it too must await future detailed computer calculation.

\section{X.3 Neutron Hazards to Other Spacecraft}

At peak engine output $(30 \mathrm{~Hz}$ ) for DT fuel and a target gain of 1000 (i.e., a target yield of 5,000 MJ), VISTA creates nearly $6 \times 10^{22} 14-\mathrm{MeV}$ neutrons every second. These neutrons leave the engine compartment isotropically (in all directions) at $1 / 6$ the speed of light. In order to reduce the flux of neutrons to a level between $3 \times 10^{8}$ and $3 \times 10^{9}$

$\mathrm{MeV}$-neutrons $\mathrm{m}^{-2} \mathrm{~s}^{-1}$ to avoid hazardous radiation exposures to astronauts, VISTA must be at least 8,000 to $36,000 \mathrm{~km}$ from unshielded personnel. Thus, astronauts on nearby spacecraft must hide behind their solar-flare shields for 6 to $12 \mathrm{hrs}$ while VISTA accelerates 8,000 to $36,000 \mathrm{~km}$ away. This hazard is only moderately reduced by using advanced target fuels such as DD or $\mathrm{D}^{3} \mathrm{He}$ (see Table XV-1).

Initial startup of VISTA from LEO or lunar orbit with DT fuel will hence depend on the number of people in these locations who might be exposed. There is no hazard for terrestrial beings, however, because the atmosphere is roughly seven neutron mean free paths thick. Further study is required to evaluate the hazard for high-flying aircraft.

\section{X.4 Exhaust and Particulate Hazards to Other Spacecraft}

This potential threat has not been investigated yet. In concept, the hazard is similar to that from other spacecraft, except VISTA's exhaust velocity is higher. If the exhaust were to impinge on a nearby surface so as to cause the exhaust to thermalize, the temperature of the gases could easily exceed tens of $\mathrm{eV}$. There is hence a potential problem associated with the heating or ablation of other surfaces if they are nearby. There is, however, no significant impact on massive spacecraft from momentum transfer from the exhaust gases. 
C. Orth, UCRL-LR-110500

\section{TRAJECTORY ANALYSIS FOR MARS MISSIONS}

\section{XI.1 Simple Approximate Analysis}

For accurate flight analysis, we used the NASTRAN computer code at the Jet Propulsion Laboratory (JPL). For simple estimates of flight times, we used an analytic trajectory code written by Charles Orth called IFRTRIP. This analytic code uses sophisticated rocket equations derived for relativistic conditions (i.e., based on an additive velocity parameter), which were then simplified for velocities much less than the speed of light. The code includes all of the systematics of the ICF spacecraft with a pulsed engine, including coasts, and computes the minimum flight time between two end points by simultaneously varying and optimizing four parameters: the total fusion energy expended during the trip, the fraction of this energy used in the outgoing leg, the jet power, and the effective specific impulse (which is the specific impulse multiplied by the square root of the jet efficiency). Additional assumptions were as follows:

1. Trajectories are one-dimensional, except that the distance traversed is specified threedimensionally.

2. The thrust chamber does not alter the debris speed.

3. Propellant is consumed at a constant linear rate while the engine is operating (i.e., not in coasts) even though the engine may be pulsed.

4. The target firing rep rate is constant throughout the mission, during engine operation.

5. The target mass including expellant is constant throughout the mission.

6. Changes in the fractional energies into $x$ rays, plasma debris, and neutrons caused by changes in target mass can be ignored.

7. All propellant is consumed by the end of the mission.

8. The distance going is identical to the distance coming back.

9. The time to spiral out from or into a planetary orbit must be added to the trip time calculated by the code.

10. There is no change in vehicle velocity during coasts (i.e., ignore acceleration or deceleration during coasts due to solar gravity).

11. There is no change in solar gravity during the trip except that which accounts for the overall change in gravitational potential, which can be included at the end points of the trajectory legs through negative velocities.

As a check on the accuracy of this code, we compared its output for a Mars mission with that obtained from the NASTRAN code, excluding the VISTA systematics (Fig. XI-1). Note that the agreement is satisfactory, considering that the JPL calculation indicates the extreme case of minimum trip time for a given "alpha" (with Isp varying along this optimized curve). This alpha parameter is the ratio of the spacecraft "dry" mass (with no propellant) and the jet power, in units of $\mathrm{kg} / \mathrm{kW}$, and is the only parameter governing this minimum trip time. Using IFRTRIP and the parameters shown in Table XI-1, we calculate a total flight time to Mars and back of 145 days, with a mass distribution as listed in Table XI-2. 
Figure XI-1: Comparison of one particular VISTA alpha curve with the exact JPL curve indicating the minimum trip time for any given alpha for any spacecraft to Mars.

Alpha Curves for Earth-Mars Roundtrip

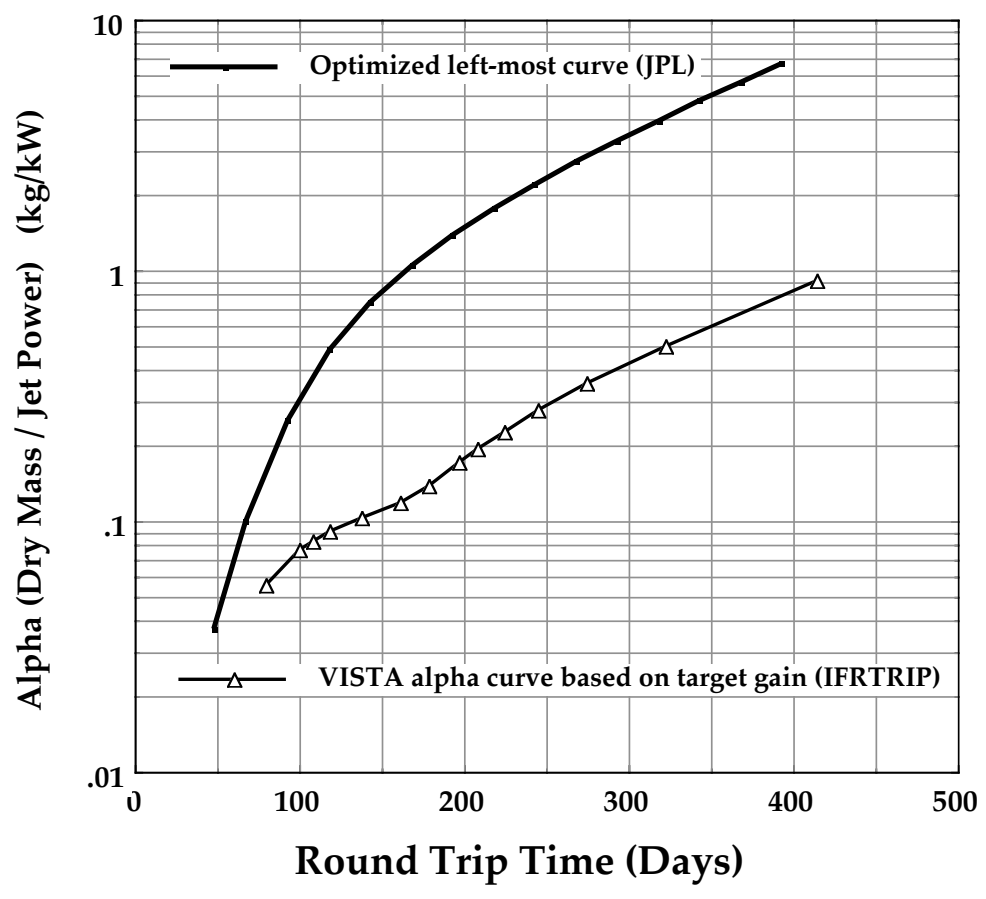

Table XI-1: Parameter Values Assumed for Advanced-Technology Case

\begin{tabular}{|c|c|c|c|}
\hline Parameter & Value & Parameter & Value \\
\hline Driver energy & $5 \mathrm{MJ}$ & $\begin{array}{l}\text { Extra } \mathrm{kg} / \mathrm{kW}_{\text {th }} \text { for micrometeoroid } \\
\text { shield }\end{array}$ & 0.007 \\
\hline Driver efficiency & $12 \%$ & $\begin{array}{l}\text { Induction electrical system } \\
\text { efficiency }\end{array}$ & $50 \%$ \\
\hline Expellant density & $0.077 \mathrm{~g} / \mathrm{cm}^{3}$ & Jet efficiency & $32 \%$ \\
\hline Expellant type & $\mathrm{H}_{2}$ & Magnet coil radius (m) & 13.0 \\
\hline Fuel type & $\overline{\mathrm{DT}}$ & Maximum pulse rate $(\mathrm{Hz})$ & 30 \\
\hline Fuel compressed $\rho \Delta \mathrm{r}\left(\mathrm{g} / \mathrm{cm}^{2}\right)$ & 5.0 & Radiator Temperature, coil (K) & 1500 \\
\hline Fuel capsule gain & 1500 & Radiator Temperature, driver (K) & 900 \\
\hline $\begin{array}{l}\text { Heat-pipe radiator } \mathrm{kg} / \mathrm{kW}_{\text {th }} @ \\
1000 \mathrm{~K}\end{array}$ & 0.07 & $\begin{array}{l}\text { Radiator Temperature, thermal } \\
\text { systems (K) }\end{array}$ & 1000 \\
\hline
\end{tabular}


Our calculations reveal that the critical parameters are the engine mass (i.e., the mass of the magnet coil/ shield and the laser driver), and the target gain (see Fig. XI-2). Note that the shapes of the curves in Fig. XI-2 reveal that it is not really necessary to have a driver efficiency much above $\sim 10 \%$ or a driver radiator temperature much above $\sim 700 \mathrm{~K}$, because little reduction in total trip time results for further increases in these parameters. It is therefore possible that a DPSSL might be used for a VISTA application, because it probably cannot exceed $700 \mathrm{~K}$ in operating temperature. On the other hand, steady improvement in performance occurs for increased target gain or improved radiator technology. Results are independent of the inter-pulse timing.

Table XI-2 Distribution of mass in metric tons for VISTA advanced-technology mission to Mars (see Table XI-4)

\begin{tabular}{|l|c|c|}
\hline \multicolumn{1}{|c|}{ Sub-system } & Mass & $\begin{array}{c}\text { Total } \\
\text { Mass }\end{array}$ \\
\hline \hline Payload System & & 289. \\
\hline Payload & 100. & \\
\hline Payload Shield & 189. & \\
\hline Propellant System & & 4458. \\
\hline Non-fuel target mass & 36.5 & \\
\hline DT fuel & 3.72 & \\
\hline Expellant $\left(0.077 \mathrm{~g} / \mathrm{cm}^{3} \mathrm{H}_{2}\right)$ & 4100. & \\
\hline Tritium refrigerator & 50. & \\
\hline Propellant tanks & 207. & \\
\hline Tritium tank & 61. & \\
\hline Driver System & & 268. \\
\hline Laser driver & 150. & \\
\hline Driver radiators & 118. & \\
\hline Thrust Chamber System & & 805. \\
\hline Coil & 216. & \\
\hline Coil shield + structure & 523. & \\
\hline Radiators & 66. & \\
\hline Auxiliary Systems & & 180. \\
\hline Startup reactor equipment & 5. & \\
\hline ALL radiator shields & 18. & \\
\hline Trusses & 42. & \\
\hline Inductor-coil power system & 115. & \\
\hline Total DRY MASS & & $\mathbf{1 8 6 0 .}$ \\
\hline TOTAL WET MASS & & $\mathbf{6 0 0 0 .}$ \\
\hline
\end{tabular}


Figure XI-2: Variation of Advanced-Technology Mission Performance to Mars With Variation in Driver Efficiency and Radiator Technology

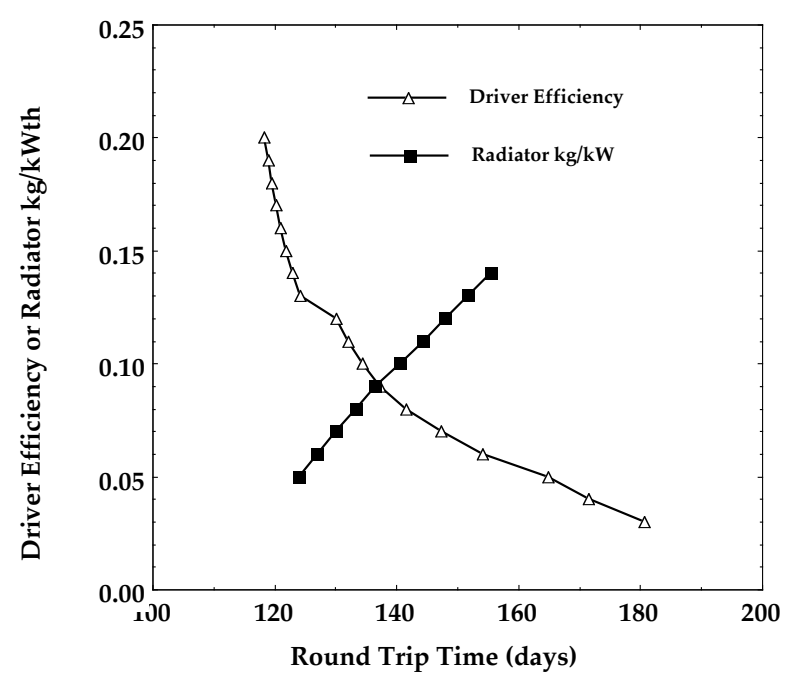

Figure XI-3: Variation of Advanced-Technology Mission Performance to Mars With Variation in Target Gain, Driver Radiator Temperature, and Payload Mass

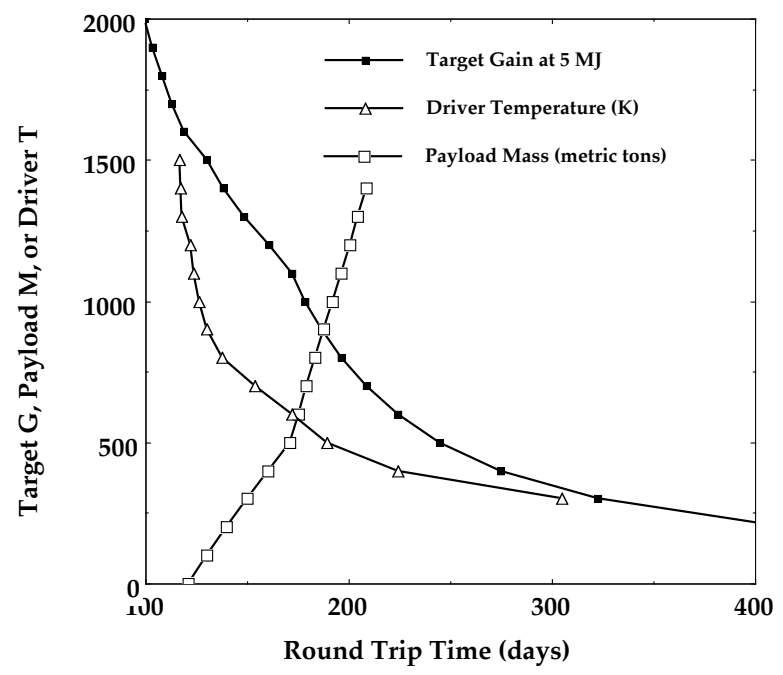




\section{XI.2 Differences From Our Previous Publications}

We have published preliminary results ${ }^{105}$ indicating Mars roundtrip mission durations of 100 to 130 days, whereas here we report 145 days. We believe the 145 -day value is more accurate, for the following reasons. We are now using a crew shield that is 50 tons larger for added crew safety. We are now including the special 61-ton tank to hold tritium at $400 \mathrm{~K}$ because of its $1 / 3 \mathrm{~W} / \mathrm{g}$ radioactive-decay heat, which we had originally forgotten to incorporate. We are now using a detailed design of the superconducting magnet coil and coil shield that has added 125 tons to the total thrust-chamber mass. On the other hand, we are now using a more realistic diode-pumped solid-state laser (DPSSL) driver that has about half the mass as the original excimer laser. We are therefore being more accurate and more detailed, and as one might expect, the more accurately the system is modeled, the more mass is typically required and the longer the missions become.

In our original (unrevised) paper for the ICENES'98 conference, ${ }^{106}$ we inadvertently labeled the 3.75 tons of DT fuel mass and the 36.7 tons of non-DT target (hohlraum) mass calculated at that time as being 40 tons of "fuel" in Table 2. This was an error (our previous publications had used the term "target materials"). We have always claimed that 3.5 to 4.0 tons of DT would be required for a roundtrip advanced-technology mission to Mars, and we support that claim here as well. Specifically, we have not changed the amount of tritium that would be required for a DT-fueled mission.

\section{XI.3 Loss in Performance due to Mass Contingencies}

It is invariably true that the launch mass for any spacecraft is always larger than that for the original design by at least $25 \%$. If we add a $30 \%$ contingency to the dry mass and also account for propellant reserves and losses, then the mission duration for a roundtrip to Mars becomes 183 days and the performance parameters change as shown in Table XI.3. Table XI.4 shows the breakdown of masses with such contingencies using the standard generic mass template adopted in 1999 by a NASA Technical Interchange Meeting. We urge all investigators to use this type of table for reporting mass distributions.

Table XI.3 Effect of mass contingencies on performance parameters for Mars Roundtrip with advanced technology

\begin{tabular}{|l|c|c|}
\hline \multicolumn{1}{|c|}{ Performance Parameter } & $\begin{array}{c}\text { Value With } \\
\text { Mass Contingency }\end{array}$ & $\begin{array}{c}\text { Value Without Mass } \\
\text { Contingency }\end{array}$ \\
\hline \hline Jet efficiency $(\%)$ & 32. & 32. \\
\hline Specific impulse, $\mathrm{I}_{\mathrm{sp}}(\mathrm{s})$ & $2.72 \times 10^{4}$ & $2.72 \times 10^{4}$ \\
\hline Effective $\mathrm{I}_{\mathrm{sp}}=\mathrm{I}_{\mathrm{sp}}\left(\varepsilon_{\mathrm{jet}}\right)^{0.5}(\mathrm{~s})$ & $1.55 \times 10^{4}$ & $1.55 \times 10^{4}$ \\
\hline Mass flow rate $(\mathrm{kg} / \mathrm{s})$ & 1.36 & 1.54 \\
\hline Thrust $(\mathrm{N})$ & $2.06 \times 10^{6}$ & $2.35 \times 10^{6}$ \\
\hline Jet power $(\mathrm{GW})$ & 15.65 & 17.80 \\
\hline One-way distance $(\mathrm{cm})$ & $2.718 \times 10^{13}$ & $2.423 \times 10^{13}$ \\
\hline Alpha $(\mathrm{kg} / \mathrm{kW})$ & 0.151 & 0.104 \\
\hline Total wet mass $($ metric tons) & 6500. & 6000. \\
\hline Mission duration (days) & 183. & 145. \\
\hline
\end{tabular}


Table XI.4 Distribution of mass in metric tons for VISTA's advanced-technology mission to Mars including contingencies (ia. = included in value above; ib. = included in value below)

\begin{tabular}{|c|c|c|}
\hline Mass Property Item & $\begin{array}{l}\text { Mass } \\
\text { Value }\end{array}$ & $\begin{array}{l}\text { Section } \\
\text { Subtotal }\end{array}$ \\
\hline \multicolumn{2}{|l|}{ I. Fusion Engine } & \\
\hline \multicolumn{2}{|l|}{ A. Input power systems } & \\
\hline Input power generators & 150. & \\
\hline Power transfer systems & ia. & \\
\hline $\begin{array}{l}\text { Radiators \& Thermal Systems (including direct } \\
\text { structure/pipes, heat exchangers, coolant, pumps, } \\
\text { refrigeration, insulation) }\end{array}$ & 104. & \\
\hline B. Fusion Chamber Wall systems & 0. & \\
\hline \multicolumn{2}{|l|}{ Vessel Wall systems } & \\
\hline \multicolumn{2}{|l|}{$\begin{array}{l}\text { Radiators \& Thermal Systems (including direct } \\
\text { structure/pipes, heat exchangers, coolant, pumps, } \\
\text { refrigeration, insulation) }\end{array}$} & \\
\hline \multicolumn{2}{|l|}{ C. Magnet systems $\quad$ (Thrust Chamber System) } & \\
\hline Magnets (Superconducting Coil) & 216. & \\
\hline Direct support structure & 16. & \\
\hline Radiation shielding & 505. & \\
\hline Thermal shielding & ia. & \\
\hline $\begin{array}{l}\text { Radiators \& Thermal Systems (including direct } \\
\text { structure/pipes, heat exchangers, coolant, pumps, } \\
\text { refrigeration, insulation) }\end{array}$ & 58. & \\
\hline D. Magnetic Nozzle systems & 0. & \\
\hline \multicolumn{2}{|l|}{ Nozzle } & \\
\hline \multicolumn{2}{|l|}{ System for adding expellant in nozzle } & \\
\hline \multicolumn{2}{|l|}{ Diverter \& other magnet systems } & \\
\hline \multicolumn{2}{|l|}{ Thrust vector control system } & \\
\hline \multicolumn{2}{|l|}{$\begin{array}{l}\text { Radiators \& Thermal Systems (including direct } \\
\text { structure/ pipes, heat exchangers, coolant, pumps, } \\
\text { refrigeration, insulation) }\end{array}$} & \\
\hline E. Fusion fuel systems & 5. & \\
\hline \multicolumn{2}{|l|}{ Target assembly } & \\
\hline \multicolumn{2}{|l|}{ Injector/Positioner } & \\
\hline \multicolumn{2}{|l|}{ Tritium breeding systems } & \\
\hline \multicolumn{2}{|l|}{$\begin{array}{l}\text { Radiators \& Thermal Systems (including direct } \\
\text { structure/ pipes, heat exchangers, coolant, pumps, } \\
\text { refrigeration, insulation) }\end{array}$} & \\
\hline \multirow{2}{*}{\multicolumn{2}{|c|}{$\begin{array}{c}\text { F. Concept-specific systems } \\
\text { Other acceleration/grid systems }\end{array}$}} & \\
\hline & & \\
\hline \multicolumn{3}{|l|}{$\begin{array}{l}\text { Radiators \& Thermal Systems (including direct } \\
\text { structure/pipes, heat exchangers, coolant, pumps, } \\
\text { refrigeration, insulation) }\end{array}$} \\
\hline \multicolumn{2}{|l|}{$\begin{array}{cc}\text { Fusion Engine Subtotal } \\
\end{array}$} & 1054. \\
\hline \multicolumn{2}{|l|}{ II. Fluids } & \\
\hline \multicolumn{2}{|l|}{ A. Main Propellants/Expellants } & \\
\hline Main impulse & 3835. & \\
\hline Flight performance reserve ( $\sim 1 \%$ of Main) & $\mathrm{ib}$. & \\
\hline Boiloff/residuals/losses ( $\sim 3 \%$ of tankable) & 271 & \\
\hline B. Stage dry ( $\geq 10 \%$ of tankable cryogenic propellants) & ia. & \\
\hline C. Adapter ( $\sim 3 \%$ of Main) & ia. & \\
\hline D. Fusion fuels & 3.5 & \\
\hline E. Other fluids & 34. & \\
\hline \multicolumn{2}{|l|}{ Fluids Subtotal } & 4144. \\
\hline \multicolumn{2}{|l|}{ III. Reaction Control Systems (included in fusion fuel sys) } & \\
\hline \multicolumn{2}{|l|}{ A. Control System } & \\
\hline \multicolumn{2}{|l|}{ B. Reaction control fluids ( $\sim 2 \%$ of Main propellant) } & \\
\hline \multicolumn{3}{|l|}{$\begin{array}{l}\text { C. Radiators \& Thermal Systems (including direct structure/pipes, } \\
\text { heat exchangers, coolant, pumps, refrigeration, insulation) }\end{array}$} \\
\hline Reaction Control Systems Subtotal & & 0 . \\
\hline IV. Tanks & & \\
\hline
\end{tabular}


C. Orth, UCRL-LR-110500

\begin{tabular}{|c|c|c|}
\hline A. Tanks (normally $\sim 15 \%$ of propellant (incl. tritium tank $=56$. ) & 263. & \\
\hline B. Insulation & ib. & \\
\hline C. Refrigeration systems (including radiators) & 50. & \\
\hline D. Pumps (including radiators) & ia. & \\
\hline E. Power systems (including radiators) & ia. & \\
\hline Tanks Subtotal & & 313. \\
\hline \multicolumn{3}{|l|}{ V. Structural Components } \\
\hline A. Primary structure (trusses, etc.) & 37. & \\
\hline B. Micrometeoroid shields (all systems) & 16. & \\
\hline C. Interface hardware & ia. & \\
\hline \multicolumn{2}{|l|}{ Structural Components Subtotal } & 53. \\
\hline \multicolumn{3}{|l|}{ VI. Payload Systems } \\
\hline A. Payload & 100. & \\
\hline B. Payload radiation \& thermal shields & ib. & \\
\hline C. Crew quarters & $\mathrm{ib.}$ & \\
\hline D. Solar-flare shields & 172. & \\
\hline E. Avionics \& communications $(\sim 0.2 \%)$ & ia. & \\
\hline $\begin{array}{l}\text { F. Radiators \& Thermal Systems (including direct structure/pipes, } \\
\text { heat exchangers, coolant, pumps, refrigeration, insulation) }\end{array}$ & ia. & \\
\hline \multicolumn{2}{|l|}{$\begin{array}{ll}\text { Payload Systems Subtotal } \\
\end{array}$} & 272. \\
\hline \multicolumn{3}{|l|}{ VII. Power (Electrical) Systems } \\
\hline A. Power conversion system & 90. & \\
\hline B. Power conditioning/processing system & 25. & \\
\hline C. Energy Storage systems & ia. & \\
\hline D. Distribution systems & ia. & \\
\hline E. Direct support structure & ia. & \\
\hline $\begin{array}{l}\text { F. Radiators \& Thermal Systems (including direct structure/pipes, } \\
\text { heat exchangers, coolant, pumps, refrigeration, insulation) }\end{array}$ & ia. & \\
\hline \multicolumn{2}{|l|}{ Power (Electrical) Systems } & 115. \\
\hline \multicolumn{3}{|l|}{ VIII. Startup/Restart Power System } \\
\hline A. Reactor & 5. & \\
\hline B. Electrical storage systems & ia. & \\
\hline C. Power conditioning/processing system & ia. & \\
\hline $\begin{array}{l}\text { D. Radiators \& Thermal Systems (including direct structure/pipes, } \\
\text { heat exchangers, coolant, pumps, refrigeration, insulation) }\end{array}$ & ia. & \\
\hline \multicolumn{2}{|l|}{ Startup/Restart System Subtotal } & 5. \\
\hline \multicolumn{3}{|l|}{ IX. Shutdown and Disposal } \\
\hline \multicolumn{2}{|l|}{$\begin{array}{c} \\
\text { Shutdown and Disposal Subtotal }\end{array}$} & 0. \\
\hline \multicolumn{3}{|l|}{ X. Total Masses (metric tons) } \\
\hline \multicolumn{2}{|l|}{ A. Total dry mass } & 1813. \\
\hline \multicolumn{2}{|l|}{ B. Total non-dry mass } & 4143. \\
\hline \multicolumn{2}{|l|}{ C. Mass growth contingency ( $30 \%$ of dry mass) } & 544. \\
\hline TOTAL WET MASS at launch & & 6500 \\
\hline
\end{tabular}

\section{XI.4 Loss in Performance due to ALL System Uncertainties}

It is important to understand that, when flight hardware is developed and a flight of VISTA occurs, there will be uncertainties in power generation, systems masses, and system performance that can each affect the roundtrip time for any mission. These uncertainties are many, including particularly the attained performance of various engine and power-system components relative to what VISTA design specifies for performance and system mass (e.g., uncertainties arising from an incomplete understanding of each system, and the mass contingencies considered in the previous section). There are also the variations from specified performance under actual flight conditions due to unforeseen or uncontrollable 
environmental factors or whatever. Of special concern is the effect of these uncertainties on jet power, radiator performance, and other system factors that can affect performance directly or indirectly through increases in vehicle wet mass. Rather than describe the details for each system mentioned in this report, we have instead made Monte Carlo runs in which EVERY system parameter for VISTA was randomly varied with its own Gaussian distribution having a standard deviation of $10 \%$. We report the effects on trip time in Table XI-5 below for Mars missions for the advanced technology case as well as for target gain of 1000 .

Table XI-5 Monte Carlo Variations in Roundtrip Trip Times to Mars For Variations of $10 \%$ in EVERY System Parameter in VISTA

\begin{tabular}{|c|c|c|}
\hline Planet Destination & Mean Target Gain & $\begin{array}{c}\text { Mean Trip Time and } \\
\text { Monte Carlo } \\
\text { Standard Deviation }\end{array}$ \\
\hline \hline Mars & 1500. & $145 \pm 18$ days \\
\hline Mars & 1000. & $184 \pm 17$ days \\
\hline
\end{tabular}


C. Orth, UCRL-LR-110500

\section{TRAJECTORY ANALYSIS FOR MISSIONS BEYOND MARS}

\section{XII.1 General Results}

A 1989 study $^{107}$ by Kathy Murray and Nathan Hoffman, two members of the original systems analysis team, was the first step in assessing the consequences for missions to planets beyond Mars. That study adapted VISTA for a mission to Titan, a moon of Saturn, with the resultant spacecraft design called "Gevaltig" (which is an approximate English pronunciation-equivalent for the German adjective "gewaltig" meaning "powerful, strong, mighty"). Considering Gevaltig to have only $\sim 60 \%$ of the dry mass of VISTA, they concluded that 500-day roundtrips to Titan would be feasible with a 100-ton payload and 1400 tons of propellant, for a total (wet) mass of 2500 tons.

Using the modern VISTA parameters, keeping the total (wet) mass near 6000 metric tons, ignoring any "spin out" to leave a planetary orbit, and using our simple systems code, we calculate the performances shown in Table XII-1 for the advanced technology case. By relaxing the restriction on total propellant mass, these flight times can be shortened by $\sim 25 \%$ at most.

Table XII-1 VISTA total roundtrip mission durations from Earth to the planets for different target gains $G$

\begin{tabular}{|c|c|c|c|}
\hline $\begin{array}{c}\text { Destination } \\
\text { Planet }\end{array}$ & $\begin{array}{c}\text { RT Time }(G=600) \\
(\text { days })\end{array}$ & $\begin{array}{c}\text { RT Time }(G=1000) \\
(\text { days })\end{array}$ & $\begin{array}{c}\text { RT Time (G=1500) } \\
(\text { days })\end{array}$ \\
\hline \hline Mercury & + & 191 & 160 \\
\hline Venus & $H$ & 163 & 108 \\
\hline Mars & 229 & 184 & 145 \\
\hline Jupiter & 632 & 486 & 422 \\
\hline Saturn & 1081 & 842 & 735 \\
\hline Uranus & 2093 & 1637 & 1425 \\
\hline Neptune & 3236 & 2527 & 2134 \\
\hline Pluto & 4087 & 3191 & 2692 \\
\hline \multicolumn{4}{|l|}{ Code didn't converge. }
\end{tabular}

These results differ from what we have previously published ${ }^{108}$ for the same reasons listed in Sec. XI.2. Note that the performance indicated in Table XII-1 allows roundtrip missions to Pluto in a little over 7 years with advanced technology. No other technology can match this performance.

\section{XII.2 Loss in Performance due to ALL System Uncertainties}

It is important to understand that, when flight hardware is developed and a flight of VISTA occurs, there will be uncertainties in power generation, systems masses, and system performance that can each affect the roundtrip time for any mission. These uncertainties are many, including particularly the attained performance of various engine and power-system components relative to what VISTA design specifies for performance and system mass (e.g., uncertainties arising from an incomplete understanding of each system, and the mass contingencies considered section XI.3). There are also the variations from specified 
performance under actual flight conditions due to unforeseen or uncontrollable environmental factors or whatever. Of special concern is the effect of these uncertainties on jet power, radiator performance, and other system factors that can affect performance directly or indirectly through increases in vehicle wet mass. Rather than describe the details for each system mentioned in this report, we have instead made Monte Carlo runs in which EVERY system parameter for VISTA was randomly varied with its own Gaussian distribution having a standard deviation of $10 \%$. We report the effects on trip time in Table XII-2 below for missions to Jupiter and Pluto for the advanced technology case as well as for target gain of 1000. The mean trip times differ slightly from those given in Table XII-1 because of the skewness of the Monte Carlo distributions.

Table XII-2 Monte Carlo Variations in Roundtrip Trip Times to Jupiter and Pluto For Variations of $10 \%$ in EVERY System Parameter in VISTA

\begin{tabular}{|c|c|c|}
\hline Planet Destination & Mean Target Gain & $\begin{array}{c}\text { Mean Trip Time and } \\
\text { Monte Carlo } \\
\text { Standard Deviation }\end{array}$ \\
\hline \hline Jupiter & 1500. & $420 \pm 26$ days \\
\hline Jupiter & 1000. & $498 \pm 35$ days \\
\hline Pluto & & \\
\hline Pluto & 1500. & $2685 \pm 180$ days \\
\hline
\end{tabular}




\section{MISSIONS BEYOND THE SOLAR SYSTEM}

For destinations beyond the outer reaches of the solar system (i.e., beyond $\sim 40 \mathrm{AU}$ ), the technology required for piloted missions of "reasonable" duration rapidly increases to points beyond what is now credible — but such missions may be possible with very advanced technology. This is easily seen in the following table, which shows the roundtrip durations for VISTA missions to points out to $10^{4} \mathrm{AU}$ for a wet mass near 6000 metric tons. Approximately $25 \%$ reductions in flight times can be realized for missions with increased propellant.

Table XIII-1 VISTA roundtrip DT missions to destinations beyond Pluto

\begin{tabular}{|c|c|c|c|c|}
\hline Item & $100 \mathrm{AU}$ & $1,000 \mathrm{AU}$ & $10,000 \mathrm{AU}$ & Alpha Centauri \\
\hline \hline Flight duration (yrs) & 18.8 & 182 & 1,805 & $4.7 \times 10^{4}$ \\
\hline Coast Velocity $(\mathrm{km} / \mathrm{s})$ & 51.5 & 52.4 & 52.7 & 52.7 \\
\hline Alpha $(\mathrm{kg} / \mathrm{kW})$ & 0.6 & 2 & 6 & 31 \\
\hline
\end{tabular}

Clearly, VISTA missions to the stars are not credible with current technology. Even at 10\% of the speed of light, it takes $\sim 80$ years to go to and return from the nearest star-and it is exceedingly difficult to get any mass up to $10 \%$ of the speed of light. This is obvious from the following discussion.

The kinetic energy of a dry mass (payload) of $\mathbf{M} \mathrm{kg}$ traveling at a fraction $\boldsymbol{\beta}$ of the speed of light $\mathrm{c}$ is given relativistically as

$$
T=M c^{2}\left\lfloor\frac{1}{\sqrt{1-\beta^{2}}}-1\right\rceil
$$

Noting that 1 jerk $=10^{9}$ joules and $\mathrm{Mc}^{2}=9 \times 10^{7}$ jerks per kilogram, we can compute how long it will take to go one-way to $10^{4} \mathrm{AU}$ as a function of $\beta$, and how much kinetic energy is involved, as follows:

Table XIII-2: Time and Kinetic Energy Required to Get to $10^{4} \mathrm{AU}$

\begin{tabular}{|c|c|c|c|}
\hline$\beta$ & $\begin{array}{c}\text { Time to go } \mathbf{~ 1 0}^{4} \mathbf{A U} \\
\text { (years) }\end{array}$ & $\begin{array}{c}\text { Kinetic energy T per kg } \\
\text { (jerks/kg) }\end{array}$ & $\mathbf{T} / \mathbf{M c}^{2}$ \\
\hline \hline 0.001 & 158 & 44.9 & $5.00 \times 10^{-7}$ \\
\hline 0.005 & 31.6 & $1,123.4$ & $1.25 \times 10^{-5}$ \\
\hline 0.01 & 15.8 & $4,494.1$ & $5.00 \times 10^{-5}$ \\
\hline 0.05 & 3.16 & $1.1255 \times 10^{5}$ & $1.25 \times 10^{-3}$ \\
\hline 0.1 & 1.58 & $4.5277 \times 10^{5}$ & $5.04 \times 10^{-3}$ \\
\hline 1.0 & 0.158 & infinity & infinity \\
\hline
\end{tabular}

Obviously, $\beta=10^{-3}$ is not fast enough because 158 years is longer than the lifetime of a human being, but $\beta \geq 0.005$ might provide an acceptable mission duration. Also, because 
the maximum energy conversion efficiency for DT fusion is (340 jerks $/ \mathrm{gm}) /\left(\mathrm{c}^{2}\right)=0.00377$, it is clear that DT fusion cannot possibly accelerate any mass to speeds greater than that corresponding to $\mathrm{T} / \mathrm{Mc}^{2}=0.00377$, or $\beta=0.0866$ at $100 \%$ burnup, unless multiple spacecraft stages are employed. Said differently, the T/ $\mathrm{Mc}^{2}$ entry for $\beta=0.1$ is beyond the reach of DT fusion without employing multiple spacecraft stages. Such an entry is essentially beyond the reach of antimatter propulsion as well, even though one might think that antimatter has $\mathrm{T} / \mathrm{Mc}^{2}=1.000$.

Nevertheless, with extremely advanced technology, there are ways to go to $10^{3}$ to $10^{4} \mathrm{AU}$ within a human lifetime. We discuss mission performance for such advanced spacecraft designs in Sec. XV.2 and Appendix A. 
C. Orth, UCRL-LR-110500

\section{DEVELOPMENT STRATEGY and TECHNOLOGY READINESS}

\section{XIV.1 Critical Development Issues}

Some of the issues that warrant consideration in future studies are listed in Table XIV-1 below. There are only two critical issues: 1) the maximum target gain that can be developed, and 2) whether the expanding plasma cools too much to make the use of a magnetic thrust chamber viable. But many other issues need consideration.

\section{Table XIV-1 Development Issues for VISTA}

\begin{tabular}{|l|l|l|}
\hline \multicolumn{1}{|c|}{ Issue } & How critical? & Comments \\
\hline \hline Thrust Chamber (Coil, Shield, Inductor) & & \\
\hline $\begin{array}{c}\text { Rate of decrease of plasma plume } \\
\text { conductivity, and effect on jet efficiency }\end{array}$ & $\begin{array}{l}\text { Potentially } \\
\text { critical }\end{array}$ & $\begin{array}{c}\text { Loss of conductivity } \\
\text { produces drag and lowers } \\
\text { jet efficiency }\end{array}$ \\
\hline $\begin{array}{l}\text { Rate of neutron damage to containment } \\
\text { vessel for Li coil shield }\end{array}$ & Moderate & \\
\hline $\begin{array}{l}\text { Rate of x-ray ablation of front surface of coil } \\
\text { shield }\end{array}$ & Moderate & \\
\hline Magnitude of hoop stress in coil structure & Moderate & \\
\hline $\begin{array}{l}\text { General effects of 30-Hz magnetic-field } \\
\text { fluctuations (eddy-current heating, EMP } \\
\text { on electronics, EMP on crew, thrust - } \\
\text { impulse effect on hot Li liquid) }\end{array}$ & Moderate & \\
\hline $\begin{array}{c}\text { Determination of the dissipation of the } \\
\text { distorted dipole magnetic field through } \\
\text { radiation or induced-current losses in the } \\
\text { conducting material of the coil shield }\end{array}$ & Moderate & \\
\hline $\begin{array}{l}\text { Optimization of the coil shield design for } \\
\text { tritium production en route }\end{array}$ & Moderate & \\
\hline $\begin{array}{l}\text { Determination of the minimum energy } \\
\text { multiplication factor for neutron and } \\
\text { gamma-ray interactions in the coil shield } \\
\text { (M < 1.15). }\end{array}$ & Slight & \\
\hline $\begin{array}{l}\text { The impact of Rayleigh-Taylor instabilities on } \\
\text { the flow of the plasma through the thrust } \\
\text { chamber }\end{array}$ & Slight & \\
\hline \hline Crew or Aircraft Safety & Moderate & \\
\hline Scattered neutron flux at cargo/ crew areas & & \\
\hline $\begin{array}{l}\text { Impact on high-flying aircraft of VISTA } \\
\text { operation in Earth orbit }\end{array}$ & Moderate & \\
\hline \hline Structural Layout & & \\
\hline Degree to which meteorite shield stops & Moderate & \\
\hline
\end{tabular}


C. Orth, UCRL-LR-110500

\begin{tabular}{|c|c|c|}
\hline $\begin{array}{l}\text { particulates but transmits radiation after } \\
\text { many months in space }\end{array}$ & & \\
\hline $\begin{array}{c}\text { Vibrational effects of } 30-\mathrm{Hz} \text { impulsive drive } \\
\text { on component lifetimes (added stress, } \\
\text { fatigue, dissipation in damping elements) }\end{array}$ & Moderate & \\
\hline \multicolumn{3}{|l|}{ Power Systems } \\
\hline $\begin{array}{l}\text { Accurate amount of mass and power needed } \\
\text { for refrigeration }\end{array}$ & Moderate & \\
\hline $\begin{array}{l}\text { Detailed design of the inductor power } \\
\text { conversion system, especially how laser } \\
\text { energy can be stored for inter-pulse period } \\
\text { ( } \geq 1 / 30 \text { second) }\end{array}$ & Moderate & \\
\hline $\begin{array}{l}\text { Detailed design of the inductor power } \\
\text { conditioning system }\end{array}$ & Moderate & \\
\hline \multicolumn{3}{|l|}{ Target/Target Systems } \\
\hline Maximum target gain $\mathrm{G}$ & $\begin{array}{l}\text { Potentially } \\
\text { critical }\end{array}$ & $\begin{array}{l}\text { G must be larger than } \\
\text { several hundred to make } \\
\text { VISTA technology } \\
\text { outperform other } \\
\text { technologies }\end{array}$ \\
\hline $\begin{array}{l}\text { Degree of difficulty in aspherically shaping } \\
\text { the expellant so as to minimize neutron } \\
\text { irradiation of spacecraft components }\end{array}$ & Minor & \\
\hline $\begin{array}{l}\text { Optional ways to optimize the performance } \\
\text { using DD or } \mathrm{D}^{3} \mathrm{He} \text { fuels, and different } \\
\text { ways to reduce the mass of tritium at } \\
\text { launch time (e.g., by using DD fuel) }\end{array}$ & Moderate & $\begin{array}{l}\text { Use of DD can reduce the } \\
\text { tritium inventory by } 3 \\
\text { orders of magnitude, but } \\
\text { reduces performance }\end{array}$ \\
\hline $\begin{array}{l}\text { Design for the tritium storage tank using } \\
\text { advanced materials }\end{array}$ & Moderate & \\
\hline Onboard tritium-handling procedures & Moderate & \\
\hline $\begin{array}{l}\text { Best method of tritium production enroute, } \\
\text { and its impact on the } 3.93 \% \text { coil-shield solid } \\
\text { angle }\end{array}$ & Moderate & \\
\hline $\begin{array}{l}\text { Design of target manufacturing/assembly } \\
\text { area }\end{array}$ & Moderate & \\
\hline \multicolumn{3}{|l|}{ DPSSL driver } \\
\hline $\begin{array}{l}\text { Determination of the maximum operating } \\
\text { temperature of a DPSSL (specifically, } \\
\text { whether the efficiency can be } \geq 6 \% \\
\text { operating at } \geq 600 \mathrm{~K} \text { with a mass excluding } \\
\text { radiators of }<300 \text { metric tons) }\end{array}$ & Moderate & \\
\hline \multicolumn{3}{|l|}{ DPSSL Optics Systems } \\
\hline $\begin{array}{l}\text { Detailed design of the final (Be) turning } \\
\text { mirrors (specifically to obtain a reflectivity }\end{array}$ & Moderate & \\
\hline
\end{tabular}




\begin{tabular}{|c|l|l|}
\hline$>80 \%)$. & & \\
\hline \hline Thermal Systems & & \\
\hline $\begin{array}{c}\text { Design of the thermal system for the two } \\
\text { final turning mirrors }\end{array}$ & Moderate & \\
\hline $\begin{array}{c}\text { Detailed design of the heat-pipe radiator } \\
\text { system }\end{array}$ & Moderate & \\
\hline $\begin{array}{c}\text { Accurate determination of how much heat } \\
\text { input the propellant tanks absorb from } \\
\text { opposing heat-pipe radiator surfaces }\end{array}$ & Moderate & \\
\hline
\end{tabular}

\section{XIV.2 Current Status}

Although it has not been well communicated to those in the scientific arena, ICF is already known to work for sufficiently large energy inputs. In particular, Halite/Centurion experiments conducted at the U.S. Nevada Test Site using energy from underground explosions to implode an inertial fusion capsule have already allowed the demonstration of excellent performance, putting to rest the fundamental questions about the basic feasibility to achieve high gain. In fact, the good performance of one capsule in this series provides a new, higher level of confidence that it will indeed be possible to design working laboratory capsules, such as those for the U. S. National Ignition Facility (NIF). The NIF is now under construction at LLNL, and is scheduled for the demonstration of target gains in the 1 to 20 range by roughly the year 2010 with $1.8 \mathrm{MJ}$ of driver energy at 350-nm wavelength. Research on the NIF is also expected to provide the needed understanding for such things as the precise requirements for shaping each laser pulse in time, how spatially smooth each focused laser beam must be (e.g., the required bandwidth for smoothing), and complete understanding of the relevant plasma instabilities inherent in the coupling of the laser energy into an indirect-drive hohlraum and generating $x$ rays to implode a capsule in a stable manner. The fast ignitor target concept, although extremely speculative, is now under intensive research that is showing some degree of promise. The fast ignitor will supposedly enable target gains well into the hundreds or more. Concepts for even higher gains have been studied, but realization of very high target gains requires future research.

Although the NIF is a solid-state flashlamp-pumped Nd:glass laser, it is directly related to DPSSLs with regard to pumping, energy storage, extraction, multi-pass amplifier architecture, light propagation, linear and nonlinear wavefront distortions, frequency conversion, temporal pulse shaping, and beam smoothing. The DPSSL approach therefore builds on the extensive solid-state laser and target development accomplished during the last two decades with Nd:glass lasers, as well as what will be accomplished at the NIF. Moreover, DPSSLs are now being built which operate in the pulse-repetition range needed for VISTA, as explained in Sec. IV.3. A 2-J DPSSL has already operated at $\leq 25 \mathrm{~Hz}$, and the 100-J 10\%-efficient Mercury laser is under construction and further development along with many other DPSSLs worldwide. Laser-diode pump sources have already been fabricated, and their design is now being optimized. Good Yb:S-FAP lasing crystals have been grown at the few-cm size, great progress having been made in understanding the sources for cracking, bubbles, and other defects. Larger crystals $(5-10 \mathrm{~cm}$ diameter) should be available in the near future. However, true scalability to the MJ size may require large doped-ceramic gain media, which are already under development, isotropy being a major issue. If isotropic ceramics can be developed, a kJ to MJ-class DPSSL may not be that far away. 


\section{XIV.3 Realistic Development Schedule and Costs}

The $\angle \$ 3 B$ cost of the NIF is being paid by the terrestrial ICF Stockpile Stewardship Program as part of the U.S. weapons (defense) programs. Much of the development cost to achieve high-gain targets will therefore be automatically covered by such agencies. The important cost for VISTA is really the cost to extend the technology to space systems.

One key advantage in using a DPSSL for VISTA is that the laser is fully modular. Such modularity not only offers redundancy for space mission reliability, but it also considerably reduces the cost of the development path. Another advantage is that a DPSSL is a solid-state laser, so it has a very mature experimental base consisting of decades of experimentation on terrestrial Nd:glass lasers. It is therefore certain that a DPSSL is the lowest-risk driver option for VISTA application.

The terrestrial (Savannah River) cost of tritium was near $\$ 30 \mathrm{k} / \mathrm{g}$, but could possibly be reduced to about $\$ 3 \mathrm{k} / \mathrm{g}=\$ 3 \mathrm{~B} /$ ton for VISTA, assuming one atom of ${ }^{235} \mathrm{U}$ can be converted to one atom of tritium for an incremental cost of $240 \$ / \mathrm{g} .{ }^{109}$ Even at the reduced rate, the 2.24 tons of tritium needed for a Mars mission would cost at least $\$ 7 \mathrm{~B}$. This expenditure could be reduced by about 3 orders of magnitude if DD fuel is used with DT hotspots. It is therefore very advantageous to use DD fuel (or even $\mathrm{D}^{3} \mathrm{He}$ ) rather than $\mathrm{DT}$, to reduce the purchase cost of the tritium to the order of $\$ 10 \mathrm{M}$.

Figure XIV-1 shows a mid-1990's plan to develop an attractive commercial IFE power plant for terrestrial use. Between then and AD 2002, the objective was to down-select the type of driver (but a DPSSL is the most developed and hence the most reliable). The plan indicates that the most important step after AD 2002 is to coordinate the operation of the National Ignition Facility (NIF), advanced driver and target R\&D, and supporting technology R\&D with results from a facility called the Integrated Research Experiment (IRE). The objectives of the IRE and these other activities are to demonstrate the integration of IFE physics at a scale reduced from that appropriate for a power plant, and to justify the scalability of the critical technologies to the next step, which is the Engineering Test Facility (ETF). The IRE must therefore convincingly demonstrate the integrated operation of a target chamber with a rep-ratable solid-state laser, complete with target injection and tracking, beam transport and focus, materials survivability, chamber clearing, and final optics protection. Most all of the dates shown in Fig. XIV-1 must now be delayed by perhaps 10 years. 


\section{Figure XIV-1}

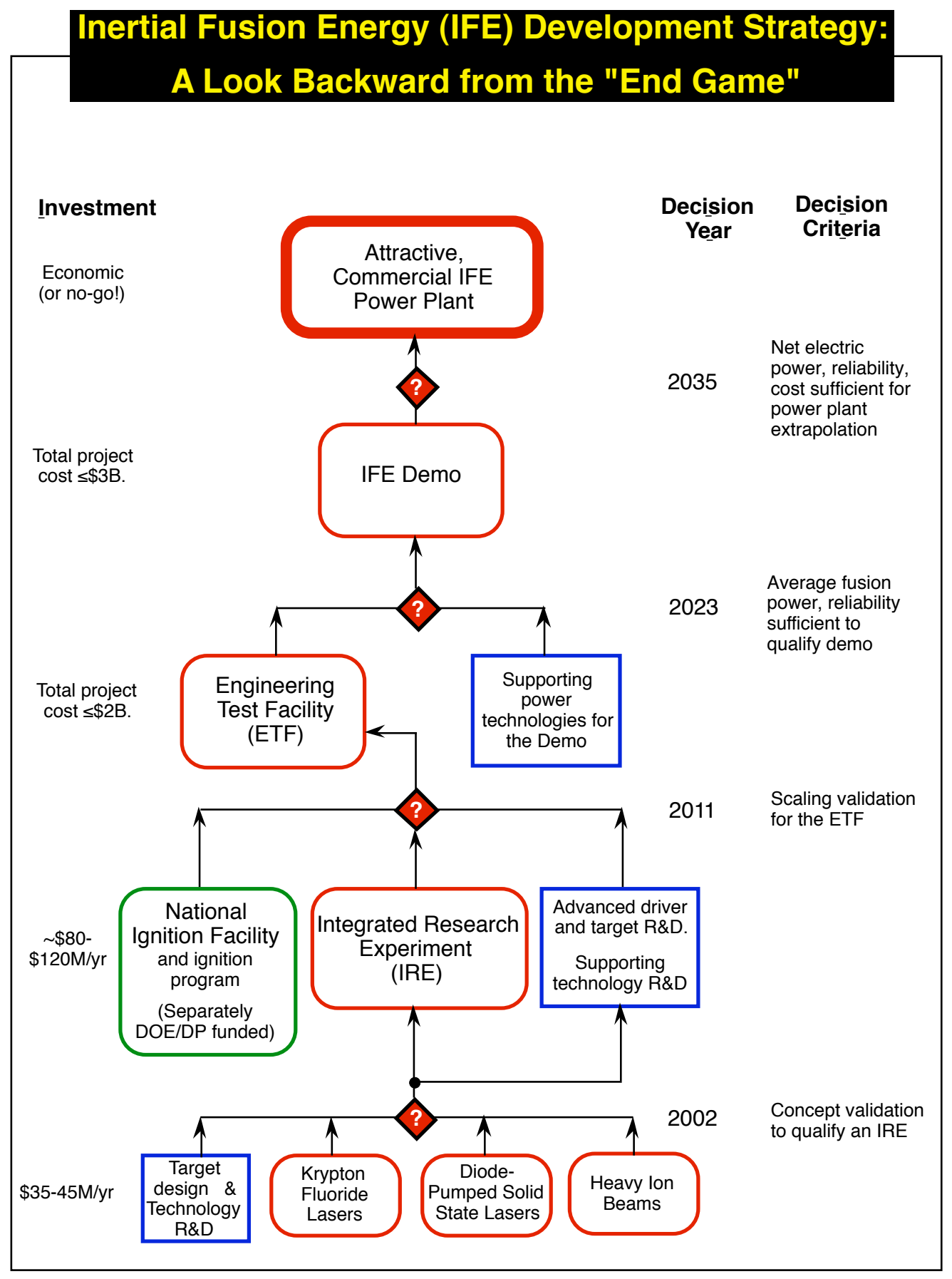

Figure XIV-2 displays one possible configuration for the IRE, namely a $4-\mathrm{kJ} 10-\mathrm{Hz}$ 2-ns 0.35- $\mu \mathrm{m}$ 0.5-THz-bandwidth DPSSL firing through a final optic in a transport tube connected to a target chamber filled with a residual gas density. Target injection could be handled by vertically dropping Xe droplets or Au targets. The laser beams striking such targets would generate $\geq 100 \mathrm{~J} / \mathrm{sr}$ of $\mathrm{x}$ rays near the normal to provide IFE-like $\mathrm{x}$-ray exposures of $7 \mathrm{~J} / \mathrm{cm}^{2}$ in a 1-keV blackbody spectrum for samples placed less than $4 \mathrm{~cm}$ from 
the targets. Beam-target coupling could be assessed with the aid of a backlighter laser. Laser operation at $1.05 \mu \mathrm{m}$ and $1 \mathrm{~kJ}$ with 1 -ps pulse widths could supply $1 \mathrm{PW}$ peak power and 10 $\mathrm{kW}$ average power for $\mathrm{MeV}$ x-ray generation. Various x-ray experiments could investigate liquid-metal film wall erosion and hydrodynamic instability of planar foils. Other experiments, coordinated with results from the NIF, could study chamber debris and gas clearing, and spin-off effects for biology, solid-state physics, plasma physics, and defense physics. A kilojoule-class DPSSL is thus a significant component of the overall program plan for energy and defense, and hence for space power applications.

\section{Figure XIV-2 One Configuration for a DPSSL Integrated Research Experiment (IRE)}

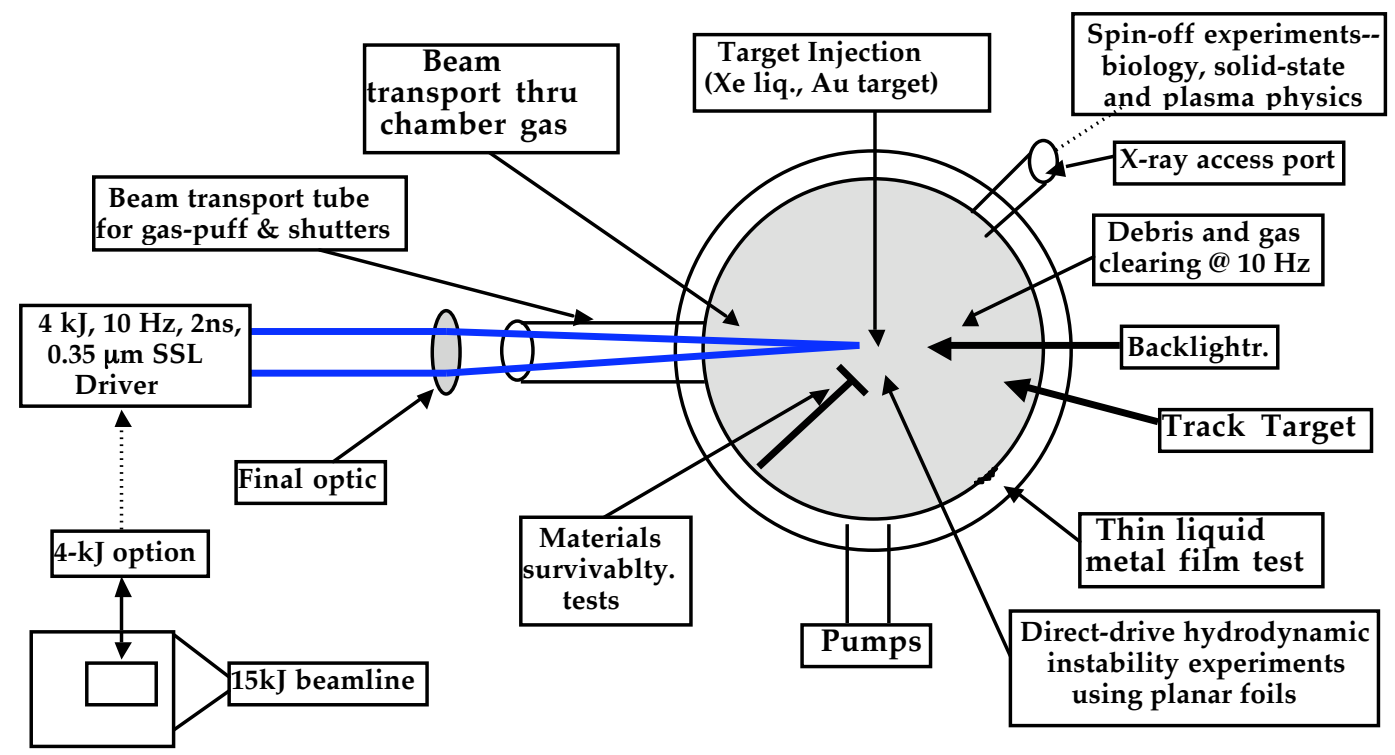

\section{XIV.4 Recommendation for Studies of Ways to Reduce Tritium Requirements}

As mentioned in detail in Sec. III.9, we recommend studying ways to reduce tritium requirements at launch time so that the 2.24 metric tons of tritium needed for a Mars mission can be reduced to as little as a few kilograms of initial tritium. Otherwise, both the time to produced the required tritium as well as the cost could easily be detriments for both the VISTA development schedule and the environmental impact of transporting so much tritium through the atmosphere. Significant reduction in the required tritium can be accomplished by using DT hotspots to burn DD or $\mathrm{D}^{3} \mathrm{He}$ fuels as described in Sec. XV.1, and by developing creative concepts such as the fast ignitor target design. With small amounts of tritium required, tritium can be produced en route, and we recommend studies to optimize the superconducting magnet shield for such a dual purpose.

Specifically, the hot $(>1500 \mathrm{C})$ liquid lithium used as a coolant for the superconducting magnet shield, collecting a solid angle of $3.93 \%$ of the neutrons emerging from the fusion reactions, can be partitioned to include a tritium-breeding zone (Fig. V-2). Because the lithium breeder zone of depth $\leq 50 \mathrm{~cm}$ would not be neutronically thick (14 $\mathrm{MeV}$ neutron mean free path is $15 \mathrm{~cm}$ ), there would be relatively little neutron slow down; hence, the reactions would be mostly high energy ones such as ${ }^{7} \mathrm{Li}\left(\mathrm{n}, \mathrm{n}^{\prime} \mathrm{He}\right) \mathrm{T}$ rather than the thermal and epithermal reactions of ${ }^{6} \mathrm{Li}(\mathrm{n}, \mathrm{He}) \mathrm{T}$. If $1 \%$ of the reactions are from the DT hotspot, then all that is needed is to have $25 \%$ of the $3.93 \% \approx 4 \%$ of the neutrons that strike 
the lithium zone breed a triton. A tritium breeding of 0.25 atoms for each neutron to strike the surface seems like a very reasonable outcome to expect. The tritium embedded in the Li could be extracted by cold trapping.

Detailed neutron transport calculations will be needed to optimize such a system and see how much tritium can practically be produced. The outcome of that study will affect the target design by determining how much tritium is available for each target. The emphasis will be on low-mass, low-maintenance equipment to do the tritium handling and target fabrication. The VISTA development schedule must include such considerations.

\section{XIV.5 Preliminary Technology Readiness Assessment}

As an aide to estimates as to when VISTA might fly, we have prepared a table showing the technology readiness levels (TRLs) for the various systems in VISTA. The TRL definitions are as follows:

\section{Technology Readiness Levels}

Level 1 -- Basic physical principles observed and reported.

Level 2 -- Technology concept and/or application formulated (e.g., through feasibility studies of concepts for engineering hardware).

Level 3 - Proof of concept for analytical or experimental critical function and/or characteristic.

Level 4 - Validation of a component and/or breadboard in a laboratory environment.

Level 5 -- Validation of a component and/or breadboard in the relevant (space) environment.

Level 6 -- Demonstration of system/subsystem model or prototype in a relevant environment (ground or space).

Level 7 -- Demonstration of a system prototype in a space environment.

We make the following assumptions, which set the time scale for the list of TRLs. Note that year ZERO is merely the year chosen for VISTA to be completely flight ready, whatever year that turns out to be (e.g., AD 2050).

\section{Assumptions For The TRLs}

- The National Ignition Facility is fully operational before year (-22).

- A full system integration test of VISTA can occur in year (-3).

- VISTA is flight operational by year ZERO.

The TRLs can then be estimated to occur at certain years as indicated in Table XIV-2. We emphasize that these years are only estimates, because politics can affect how fast IFE is developed. Actual dates indicate when the TRL was accomplished; otherwise, the time is specified relative to year zero for TRL 7. 
Table XIV-2 Technology Readiness Level Estimates

\begin{tabular}{|l|c|c|c|c|c|c|c|}
\hline \multicolumn{1}{|c|}{ VISTA Item } & TRL 1 & TRL 2 & TRL 3 & TRL 4 & TRL 5 & TRL 6 & TRL 7 \\
\hline \hline DRIVER & & & & & -6 & -3 & 0 \\
\hline Low-mass high-temperature DPSSL & $1972-75$ & 1996 & 2000 & -12 & & & \\
\hline Low-damage 0.3 or 0.5- $\mu$ m optics & $1980 \mathrm{~s}$ & $1980 \mathrm{~s}$ & 1993 & 2010 & & & \\
\hline Lifetime of 108 repetitions & $1980 \mathrm{~s}$ & $1980 \mathrm{~s}$ & $1990 \mathrm{~s}$ & -10 & & & \\
\hline TARGET & & & & & -6 & -3 & 0 \\
\hline Working high-gain targets & $1980 \mathrm{~s}$ & $1990 \mathrm{~s}$ & 2010 & 2015 & & & \\
\hline Target gains of 1000 & 2010 & 2012 & -15 & -10 & & & \\
\hline Integrated target fabrication & $1980 \mathrm{~s}$ & $1990 \mathrm{~s}$ & $1990 \mathrm{~s}$ & -7 & & & \\
\hline Target injection/ tracking system & $1980 \mathrm{~s}$ & $1990 \mathrm{~s}$ & 2005 & -7 & & & \\
\hline MAGNETIC THRUST CHAMBER & & & & & -5 & -3 & 0 \\
\hline 12-T (warm) superconducting magnet & $1980 \mathrm{~s}$ & $1990 \mathrm{~s}$ & $1990 \mathrm{~s}$ & -15 & & & \\
\hline Chamber plasma transport & 1986 & 1987 & 2008 & -10 & & & \\
\hline Efficient plume decoupling & 1986 & 1987 & 2008 & -7 & & & \\
\hline 0.07-kg/kWt HEATPIPE RADIATORS & $1980 \mathrm{~s}$ & $1990 \mathrm{~s}$ & 2005 & -20 & -10 & -3 & 0 \\
\hline INDUCTOR POWER SYSTEM & $1800 \mathrm{~s}$ & 1987 & 2010 & -14 & -8 & -3 & 0 \\
\hline STRUCTURE AND MISCELLANEOUS & & & & & -8 & -3 & 0 \\
\hline Micrometeoroid shield & $1980 \mathrm{~s}$ & $1990 \mathrm{~s}$ & $1990 \mathrm{~s}$ & -15 & & & \\
\hline Crew quarters & $1990 \mathrm{~s}$ & $1990 \mathrm{~s}$ & $1990 \mathrm{~s}$ & -10 & & & \\
\hline Fuel tanks & $1990 \mathrm{~s}$ & $1990 \mathrm{~s}$ & $1990 \mathrm{~s}$ & -10 & & & \\
\hline Startup fission reactor & $1990 \mathrm{~s}$ & $1990 \mathrm{~s}$ & $1990 \mathrm{~s}$ & -10 & & & \\
\hline
\end{tabular}

\section{XIV.6 A Fast-Track Approach to Fusion Propulsion}

\section{XIV.6.1 Synergism Possible From a NASA-DOE Dual Approach}

The time estimates given above have no absolute scale for the completion dates, because of the variability possible from the level of national commitment, financial uncertainties, politics, et cetera. Nevertheless, one might ask whether VISTA could be developed to launch in, say, 20 to 25 years after the start of a really "dedicated" NASA "fusion propulsion program." In other words, if the nation were really committed to such an endeavor, could VISTA be launched in 20 years? Of course, 20 years is less than half the time one would normally allot for the development of fusion space propulsion if NASA were to wait for DOE to develop inertial fusion technologies before initiating its own dedicated fusion propulsion program.

Significant shortening of the expected time scale is possible, however, based on a two-pronged NASA-DOE approach directed at developing in parallel both (1) the specific space applications as well as the applicable propulsion technologies needed for VISTA, as funded primarily by NASA, and (2) the physics and engineering concepts for terrestrialbased ICF technologies, as funded primarily by DOE. This approach can be successful because of the synergism expected with both NASA and DOE expending efforts in fusion science and technology for their respective applications. The crux is for NASA to extract certain key technologies from DOE at appropriate times, and proceed to meet certain milestones in a parallel, not series, effort with DOE's terrestrial program. DOE research would also prosper by a similar extraction of technology developed in the NASA-funded path. In this way, technical information on underlying fusion science and technology could 
flow in both directions between NASA and DOE to the benefit of both the space program and the terrestrial program.

\section{XIV.6.2 Differences Between Terrestrial and Space Applications}

The technical and programmatic requirements for fusion propulsion have significant differences as well as similarities from those of terrestrial energy production. Some of the differences conceivably could make the propulsion application of fusion easier technically, while some might make it more challenging. For example, the fusion cycle can be operated in an open (uncontained) mode for propulsion, whereas the operating cycle must necessarily be closed (contained) for energy generation. This difference could potentially lead to entirely different fusion approaches that are far easier to accomplish for propulsion than for energy applications. In addition, the naturally occurring vacuum environment of space is a big advantage for many fusion systems. On the other hand, space applications require much more stringent adherence to low mass, compactness, and insensitivity to vibrations or positional precision. These factors are not direct design considerations in the DOE program except insofar as they might impact the cost of the power plant. Highly creative innovations that might represent fundamental departures from the terrestrial program are therefore anticipated for the propulsion application.

In addition to the technical differences, DOE's terrestrial fusion energy program is driven programmatically by the economics of the terrestrial cost of energy (pennies per $\mathrm{kW}-\mathrm{hr}$ ) and by the absence of a global energy crisis for perhaps another 50 years. The latter tends to set the time frame for DOE's technical program. On the other hand, NASA could use fusion propulsion right now to great advantage, if it were available. In a sense, NASA is the more anxious customer for fusion.

Because of these differences between the terrestrial and propulsion applications, if NASA wishes to have fusion propulsion be a reality, it needs to take full responsibility for developing the technology in areas where it can (and therefore should). While doing so, it must maintain close collaboration with DOE and other technical partners by utilizing, leveraging, synergizing, complementing, and supplementing the DOE fusion efforts and resources.

\section{XIV.6.3 Shifting Toward a Fast-Track Approach}

Table XIV-3 describes a generalized DOE roadmap for the development of inertial fusion energy (IFE) systems for terrestrial power production using inertial confinement fusion (ICF) with a heavy-ion, $\mathrm{KrF}$, or diode-pumped solid-state laser (DPSSL) driver. This table is based on the fact that ICF has already been shown to work for sufficient energy input. Investigators are therefore reasonably confident that the U. S. National Ignition Facility (NIF) can demonstrate ignition and propagating burn in DT in the laboratory near the threshold for ignition to establish the minimum driver energy needed for an IFE power plant.

Because more facilities than just the main fusion facilities are required at each level to develop supporting technologies (such as 14-MeV-neutron sources for materials development), at least two projects ("machines") would probably be built for each level for IFE. The total facility construction cost to develop terrestrial fusion power is thus going to be at least $\$ 10 \mathrm{~B}$ to $\$ 20 \mathrm{~B}$, and involve a duration of as much as 60 years. Consequently, to enable the launch of a fusion propulsion system in a shortened time scale, a more-efficient, enhanced route to success must be accomplished in the areas that are specific to fusion propulsion. These areas must hence have associated "milestones" that keep the program on 
schedule. There are also associated "key decision points" that must be reached to enable the milestones to be met.

Table XIV-3 One Possible DOE Roadmap for IFE

\begin{tabular}{|c|c|c|c|c|}
\hline Level & Description & $\begin{array}{l}\text { Typical One- } \\
\text { Project Cost } \\
\text { (\$B) }\end{array}$ & $\begin{array}{l}\text { Typical } \\
\text { Project } \\
\text { Duration } \\
\text { (years) }\end{array}$ & $\begin{array}{c}\text { Typical } \\
\text { Operating } \\
\text { Cost } \\
(\$ M / y r) \\
\end{array}$ \\
\hline $\begin{array}{l}\text { Validate } \\
\text { concepts }\end{array}$ & $\begin{array}{l}\text { Beam-line experiments to } \\
\text { validate and down- } \\
\text { select concepts for an } \\
\text { IRE }\end{array}$ & 0.3 to 0.4 & 5 to 10 & $\overline{50}$ \\
\hline $\begin{array}{l}\text { NIF + Integrated } \\
\text { Research } \\
\text { Experiment } \\
\text { (IRE) }\end{array}$ & $\begin{array}{l}\text { Demonstration of ignition } \\
\text { and energy gain in the } \\
\text { laboratory, and } \\
\text { validation of scaling to } \\
\text { the ETF }\end{array}$ & 3 & 10 to 15 & 80 to 120 \\
\hline $\begin{array}{l}\text { Engineering Test } \\
\text { Facility (ETF) }\end{array}$ & $\begin{array}{l}\text { Down-selection and } \\
\text { demonstration of } \\
\text { average fusion power } \\
\text { with sufficient reliability } \\
\text { to qualify a DEMO }\end{array}$ & $\begin{array}{l}\text { At least } \\
2 \text { to } 3\end{array}$ & 10 to 15 & 100 to 200 \\
\hline $\begin{array}{l}\text { Engineering } \\
\text { DEMO }\end{array}$ & $\begin{array}{l}\text { Demonstration of net } \\
\text { electric power, } \\
\text { reliability, and cost to } \\
\text { qualify POWER PLANT }\end{array}$ & 3 to 4 & 15 to 20 & 200 to 300 \\
\hline
\end{tabular}

Table XIV-4 lists one possible set of milestones and key decision points that might enable development in a shortened time scale of roughly 20 years. We first note that NASA funding will undoubtedly permit research on many different propulsion concepts, not just VISTA's [e.g., Innovative Confinement Concepts (ICCs) like magnetized target fusion (MTF) might also be involved]. In such a case, the down-selection to one concept per mission type might be moved back to as late as year 15 in Table XIV-4, but no concept can be researched if it doesn't pass a "proof of principle" demonstration. This demonstration must include proof that the fusion process can indeed produce a propagating burn with net (ICF) energy gain using a practical fusion fuel (DT). In particular, contrary to the common practice in the DOE development path, the "proof of principle" demonstration cannot consist of an experimental verification at a lower performance plus credible computer simulations to extrapolate the performance to a real net energy gain. The reason for demanding such "proof" here is that we must be this strict in order to meet the 20-year timeline. Specifically, for an ICF "proof of principle" experiment, this means that we require net energy gain from the capsule, but the capsule need not be injected at high speed and imploded "on the fly." 
C. Orth, UCRL-LR-110500

Table XIV-4 ICF Fusion-Propulsion Milestones and Key Decision Points

\begin{tabular}{|c|c|c|}
\hline $\begin{array}{l}\text { Years After NASA } \\
\text { Program Start } \\
\end{array}$ & $\begin{array}{c}\text { Key Decision Points } \\
\text { (Yes/No) }\end{array}$ & Milestones \\
\hline & 1. "Proof of principle" demonstrated? & \\
\hline \multirow[t]{3}{*}{5} & & $\begin{array}{l}\text { 1. Demonstration of a } \\
\text { propagating fusion burn using } \\
\text { a practical fusion fuel }\end{array}$ \\
\hline & $\begin{array}{l}\text { 2. Nozzle shown to meet thrust } \\
\text { requirements in an EDGT*? }\end{array}$ & \\
\hline & $\begin{array}{l}\text { 3. Flight fusion system shown to meet } \\
\text { steady-state physics requirements } \\
\text { (n } \tau \mathrm{T} \text {, etc.) in an EDGT*? }\end{array}$ & \\
\hline 12 & & $\begin{array}{l}\text { 2. Successful ground engineering } \\
\text { test of integrated fusion } \\
\text { systems }\end{array}$ \\
\hline \multirow[t]{2}{*}{15} & $\begin{array}{l}\text { 4. Choice made to develop (i.e., "cut } \\
\text { metal" on) only one fusion concept } \\
\text { per mission type (down-selection)? }\end{array}$ & \\
\hline & $\begin{array}{l}\text { 5. Flight hardware developed (i.e., } \\
\text { designed and a prototype built)? }\end{array}$ & \\
\hline \multirow[t]{2}{*}{18} & & $\begin{array}{l}\text { 3. Successful ground engineering } \\
\text { test of integrated flight and } \\
\text { fusion systems }\end{array}$ \\
\hline & $\begin{array}{l}\text { 6. Adequate } \mathrm{ETO}^{* *} \text { mass capability } \\
\text { developed? }\end{array}$ & \\
\hline 20 & & 4. Integrated flight test \\
\hline
\end{tabular}

The tough milestone to meet is the demonstration by year 5 of a propagating fusion burn. The NIF would be capable of such demonstration, but certainly not before the year 2006 (or perhaps even 2010). Seven years after the completion of milestone \#1, NASA must demonstrate milestone \#2, which is a successful ground engineering test of the integrated fusion system. Such a test demands that two key decision points be reached: a successful demonstration of the required physics parameters in terms of the Lawson criterion $(n \tau T=$ product of burn density, confinement time, and burn temperature) and a successful demonstration that the nozzle for each concept can indeed meet the projected thrust requirements for the missions involved. The integration of the fusion system therefore relies on successful demonstration of the two key subsystems of the fusion system: the basic engine compartment (i.e., fusion chamber), and the nozzle. Operation of the fusion chamber requires sufficient energy input from laser beams, and sufficient fuel or capsule input for sustained operation. These subsystems must therefore be developed slightly faster than the level achieved in the DOE-funded R\&D program. Separate identification of the nozzle subsystem can be rather independent of the DOE program, but is especially important here because it determines the level of thrust. Said differently, the nozzle can have its own problems with such things as drag from sluggish decoupling from nozzle magnetic fields or lowered jet efficiency from non-thermalized (hybrid) mass flows. NASA must therefore emphasize achievement of the proper nozzle projected thrust. 
After down-selection to one fusion concept per mission type, NASA must develop the flight hardware (i.e., "cut metal" by designing and building at least one prototype) and be ready for milestone \#3, which is a successful integrated test of the combined flight hardware and fusion systems on the ground. In the mean time, NASA must ensure that it will have the needed heavy-lift launch vehicle or its equivalent, whatever is required, to assemble the fusion-propulsion systems in Earth orbit. Only then can the actual flight test occur.

\section{XIV.6.4 Fast-Track R\&D Roadmap}

Figure XIV-3 shows the layout of the above milestones and key decision points, as well as the interfaces between NASA and DOE for a fast-track approach. Note that the DOE timeline at the bottom of this figure has been listed differently than in Table XIV-4. This was done for two reason: to combine MFE and IFE levels into one generic DOE listing, and to highlight the features important to NASA. The nature of the lines drawn on the figure indicate the type of funding and the federal agency involved, as explained in the legend at the bottom.

The Integrated Performance Extension box shown along the DOE timeline is a combination of the Performance Extension for MFE systems and the NIF/IRE level for ICF systems. This box is meant to show interim achievement along the way to the development of an ETF facility. The construction and operation of the ETF, however, is not required for NASA to attain a launch of a fusion propulsion system, as shown. Only the inputs indicated with arrows proceeding from the DOE line to NASA key decision points and milestone boxes are required for NASA to benefit from the DOE-funded research. DOE, in turn, can prosper from the accelerated NASA path, but arrows indicating this technology transfer are not shown for reasons of clarity.

Figure XIV-3 thus shows the main interface connections between DOE and NASA, at which times NASA must rely on the R\&D achievements of the terrestrial research. This figure therefore illustrates how the DOE development of fusion for terrestrial power plants can proceed at a more modest rate while NASA develops fusion for space propulsion along a faster parallel path. It is important to realize to what extent this approach is dependent on the DOE research path, and how the appropriately timed extractions of DOE technology can offer NASA the opportunity to develop a fusion propulsion application at an accelerated rate. 


\section{Roadmap for Development of Nuclear Fusion Propulsion}

\section{Figure XIV-3}

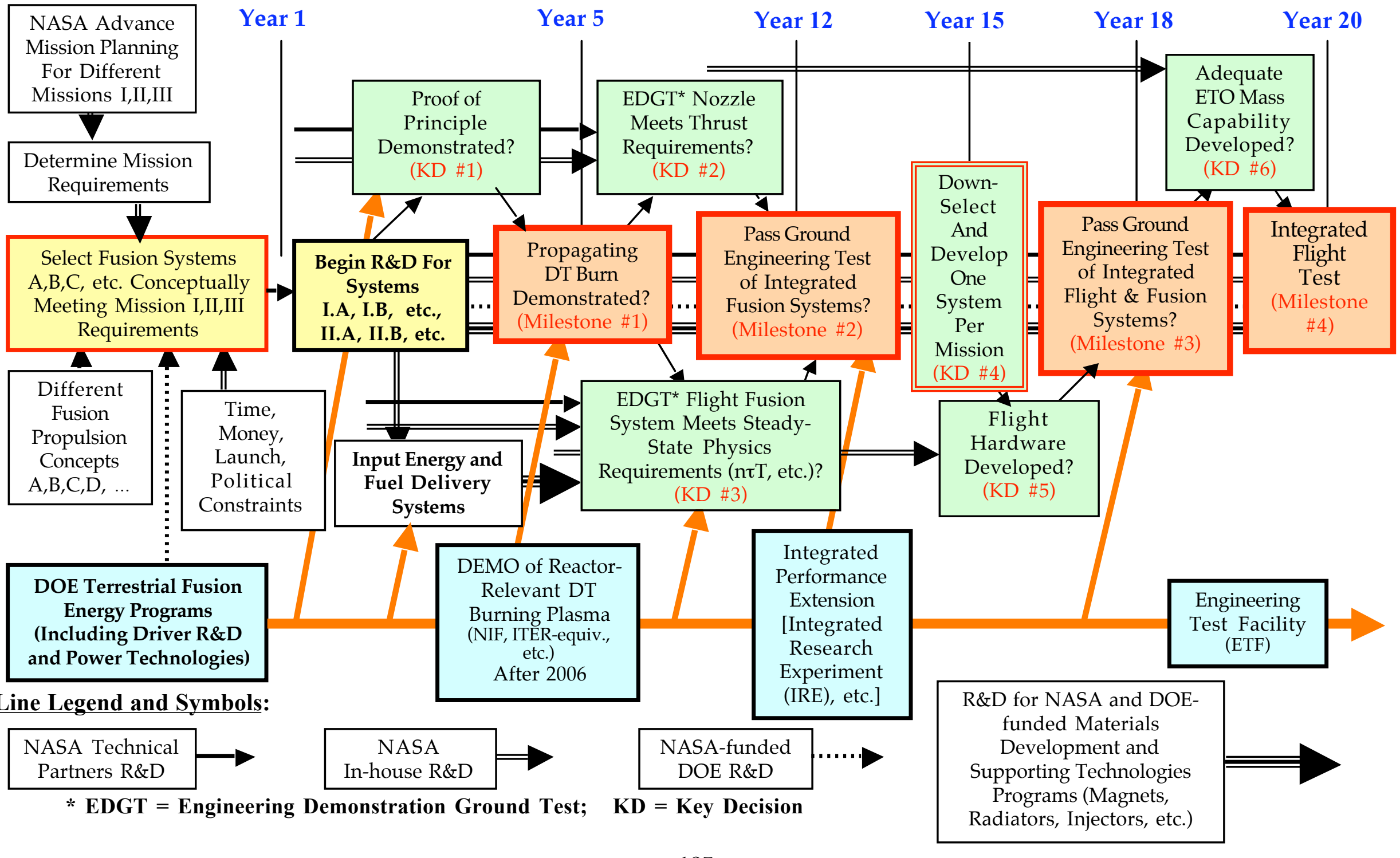




\section{XIV.6.5 NASA R\&D Funding Profile}

A truly accurate determination of NASA's funding requirements to develop fusion propulsion as outlined in Fig. XIV-3 will take a great deal more study than can be represented here. Nevertheless, we can provide a rough estimate of the minimum funding profile by making the following four assumptions:

1. DOE will provide essentially all of the funding to accomplish the "proof of principle" experiments.

2. Wherever possible, NASA will accomplish its key decisions and milestones using demonstration experiments attached to and run "piggy-back" on DOE experimental facilities (the NIF, etc.), so NASA will not have to build separate facilities.

3. NASA must fund a significant portion of the expense for the development of subsystems that DOE would eventually fund, but required by NASA at times sooner than required in the DOE path (e.g., accelerators for ICF capsule injection, and kilojoule beam lines representing the final scaling to full ICF driver energies).

4. NASA must fund the development of the technologies specific to space applications (e.g., the nozzle tests, the development of the flight hardware, and the ETO launch capability).

With these assumptions, we can estimate the minimum direct-cost funding that NASA must add to planned DOE funding to achieve the key decisions and milestones outlined in Figure XIV-3. The results are shown in Table XIV-5.

Table XIV-5 Minimum Direct-Cost Funding Base (in AD 2000 \$) for NASA's Fast-Track Development of Fusion Propulsion

\begin{tabular}{|c|c|}
\hline Item & $\begin{array}{c}\text { Minimum Direct } \\
\text { Cost (\$M) }\end{array}$ \\
\hline \hline KD \#1: Proof of Principle & 10. \\
\hline Milestone \#1 Burning Plasma Demo & 20. \\
\hline KD \#2: Nozzle Thrust Test & 150. \\
\hline KD \#3: Steady-State Physics Demo & 50. \\
\hline Milestone \#2: Integrated Fusion System Test & 260. \\
\hline KD \#4: Down-selection & 10. \\
\hline KD \#5: Flight Hardware Development & 100. \\
\hline Milestone \#3: Integrated Test of Flight and Fusion systems & 400. \\
\hline Total & $\mathbf{1 , 0 0 0 .}$ \\
\hline
\end{tabular}

Figure XIV-4 displays an estimated funding profile for these outlays, in terms of the number of years after the start of a dedicated NASA Fusion Propulsion Program. 
Figure XIV-4 Estimated NASA Fast-Track Direct-Cost Funding Profile

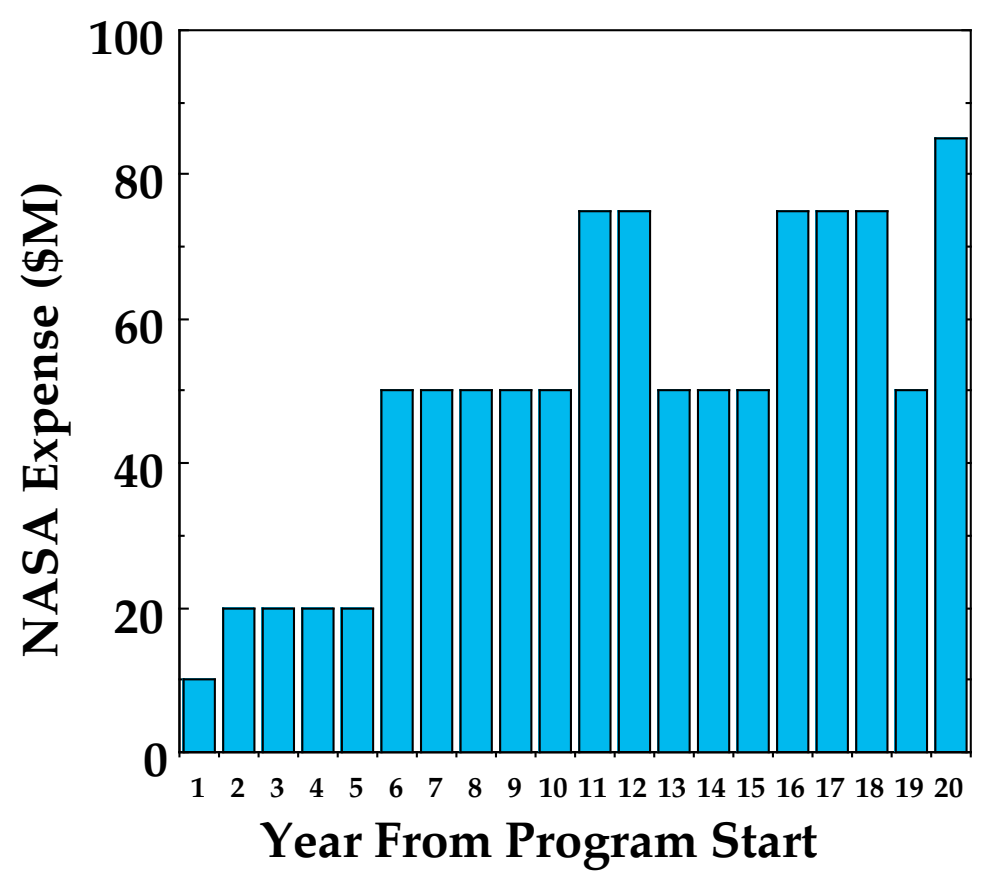

As is evident from Table XIV-5 and Figure XIV-4, the total minimum direct-cost expenditure by NASA is estimated to be $\$ 1 \mathrm{~B}$ in AD 2000 dollars. The actual expense could be considerably more than this if NASA is unable to arrange its planning according to the four assumptions listed above. Nevertheless, we conclude that a Fast-Track approach need not require many billions of dollars to accomplish, if planned and executed appropriately. Any indirect costs must of course be added to these direct-cost estimates. 
C. Orth, UCRL-LR-110500

\section{ADVANCED-TECHNOLOGY CONSIDERATIONS}

\section{XV.1 Near-Future Advancements}

There are many elements of advanced technology that could be considered for future VISTA studies, as evidenced by the following questions:

1. Can the expellant mass be changed during a roundtrip in order to maximize the initial acceleration, further reduce the trip time, etc.?

2. Can the shape of the expellant be adjusted to promote exhausting through the thrust chamber, or to promote rifling of the target?

3. Can at least a small part of the power generated onboard come from the low-temperature waste heat sent to the radiator system?

4. Can more advanced radiator designs be implemented?

5. Can advanced fuels be used without degraded target gains?

If advanced fuels like DD or $\mathrm{D}^{3} \mathrm{He}$ can be used (\#5 above), then VISTA performance would normally be reduced as listed in Table XV-1 below, because of the reduced target gains. These results assume a total vehicle mass of 6,000 metric tons. However, the advanced fuels, ignited with a hot spot of DT, require three orders of magnitude less onboard tritium, and are therefore highly desirable.

Table XV-1 Mars Missions With a 5-MJ Driver and Different Target Fuels

\begin{tabular}{|l|c|c|c|}
\hline \multicolumn{1}{|c|}{ Item } & DT fusion & DD fusion & $D^{3}$ He fusion \\
\hline \hline Minimum Mars roundtrip time & 145 days & 261 days & 241 days \\
\hline Mass of tritium used & $2237 \mathrm{~kg}$ & $2.206 \mathrm{~kg}$ & $2.074 \mathrm{~kg}$ \\
\hline Engine alpha $(\mathrm{kg} / \mathrm{kW})$ & 0.104 & 0.41 & 0.33 \\
\hline Vehicle mass ratio & 3.23 & 3.97 & 3.96 \\
\hline Jet power & $17.8 \mathrm{GW}$ & $3.64 \mathrm{GW}$ & $4.56 \mathrm{GW}$ \\
\hline Thrust & $0.23 \mathrm{MN}$ & $0.092 \mathrm{MN}$ & $0.106 \mathrm{MN}$ \\
\hline Effective specific impulse & $15.5 \mathrm{ks}$ & $8.1 \mathrm{ks}$ & $8.8 \mathrm{ks}$ \\
\hline Total number of emitted neutrons & $1.04 \times 10^{29}$ & $1.08 \times 10^{29}$ & $0.15 \times 10^{29}$ \\
\hline
\end{tabular}

Note that there is only marginal advantage of $\mathrm{D}^{3} \mathrm{He}$ over $\mathrm{DD}$ - there is a significant lowering of the total neutron irradiation of nearby spacecraft, but otherwise, the two advanced fuels have similar performance. Therefore, if ${ }^{3} \mathrm{He}$ proves to be difficult to obtain, as is expected, and if a suitable cryogenic state of $D^{3} H e$ proves to be unattainable, as is expected, there is no need to pursue the use of $\mathrm{D}^{3} \mathrm{He}-\mathrm{DD}$ is sufficient.

Most everyone might think that antimatter fuel would be the ultimate fuel for VISTA, but this is probably not the case. Antimatter-matter annihilations release energy in the form of charged pions and neutral pions, the latter decaying into gamma rays. These pions have energies of $\sim 1 \mathrm{GeV}$, and hence have very long mean free paths in material $\left(\sim 140 \mathrm{~g} / \mathrm{cm}^{2}\right)$. Thus, less than $4 \%$ of the tremendous energy produced can interact in target material to 
produce plasma debris for redirection by the magnetic thrust chamber. Consequently, the performance with antimatter fuel is much worse than that with DT fuel even though the mass-conversion efficiency for antimatter fuel is unity (see Table XV-2). This inability of the antimatter products to interact quickly requires a mass of antimatter $\left(5 \times 10^{27}\right.$ antiprotons) that is enormous in comparison to even extrapolated antimatter production rates. Future studies may want to address how it might be possible to apply antimatter fuels to VISTA.

Table XV-2 Mars Missions With DT or Antimatter Fuel

\begin{tabular}{|l|c|c|}
\hline \multicolumn{1}{|c|}{ Item } & DT fusion & Antimatter \\
\hline \hline Minimum Mars roundtrip time & 145 days & 220 days \\
\hline Fuel mass required (DT or antiprotons) & $3728 \mathrm{~kg}$ & $9.07 \mathrm{~kg}{ }^{*}$ \\
\hline Engine alpha $(\mathrm{kg} / \mathrm{kW})$ & 0.104 & 0.25 \\
\hline Vehicle mass ratio & 3.23 & 3.71 \\
\hline Jet power & $17.8 \mathrm{GW}$ & $6.6 \mathrm{GW}$ \\
\hline Thrust & $0.23 \mathrm{MN}$ & $0.13 \mathrm{MN}$ \\
\hline${\text { Effective } \mathrm{I}_{\text {sp }}}^{*}{ }^{*}$ Plus an equal mass of normal matter. & $15.5 \mathrm{ks}$ & $10.0 \mathrm{ks}$ \\
\hline
\end{tabular}

\section{XV.2 Far-Future Advancements}

Appendix A details how the VISTA concept can be extended for extremely high performance using extremely advanced spacecraft designs. We consider a one-way mission with a stop at the destination, and derive scaling relationships that detail how mission performance depends on the critical parameters. In particular, for minimum trip times, we show the following:

1. The optimum exhaust velocity is about twice the average mission velocity (not the same velocity).

2. At constant radiated power, there is a tradeoff between exhaust velocity and thrust, with higher exhaust velocity leading to lower thrust and vice versa, because their product is proportional to the radiated power through factors that depend on technology. For example, the price of light-speed exhaust is low thrust per unit engine power, and hence low performance for missions that do not approach light-speed.

3. Minimum-time missions always involve a coast period. Loading on more fuel to keep the engine on longer is counterproductive, because the increased mass leads to smaller accelerations and a longer trip time.

4. The ratio of coast to average velocity is exactly $3 / 2$.

5. Every minimum-time mission accelerates, coasts, and decelerates for the same fractions of the total trip time.

6. Every minimum-time mission has the same ratio of peak velocity to mean velocity.

7. There is an optimum radiated power for a given distance, and the trip time increases if the ship's power dissipation is increased above this optimum. This counter-intuitive result occurs because the radiator mass required to dissipate the absorbed power rises faster than the increase of performance with increased available power.

8. The optimum spacecraft design includes planar radiators. The optimum high-performance spacecraft hence looks like an incandescent $(1500 \mathrm{~K})$ planar object. 
For the extreme case of a 3-metric-ton spacecraft (including structure, engine, radiators, micro-meteoroid protection, and payload), Appendix A reveals performances of 16 days to $1 \mathrm{AU}$, less than 1 year to $100 \mathrm{AU}, 4.3$ years to $1,000 \mathrm{AU}$, about 20 years to $10^{4} \mathrm{AU}$, and 180 years to Alpha Centauri. 


\section{COMPARISON WITH OTHER TECHNOLOGIES}

In conducting this study, it has become very evident to us that the accurate assessment of mission durations, and hence the real viability of any concept, rests fundamentally on the degree to which a study addresses the details of the systems involved. The more we investigated each subsystem in depth, the more massive VISTA became. For example, avoiding x-ray ablation of the coil shield doubled the size of the spacecraft. Making sure there was adequate protection from micrometeoroids can cut the thermal efficiency of the heat-pipe radiators in half!

We therefore have to add a large "giggle factor" to anyone suggesting a conceptual design based on a rocket equation and not on a detailed systems analysis, especially if they claim ${ }^{110}$ piloted transport roundtrip missions of fewer than 100 days to Mars without considering the concepts described in Appendix A! Nevertheless, we understand that a concept can be released before a systems analysis is completed, so we make a few general remarks in the following Sections comparing VISTA to alternative technologies.

\section{XVI.1 Magnetic Confinement Fusion (MCF)}

We will assume for this report that MCF will eventually be demonstrated, even though ICF has been shown to work and MCF has not. MCF then faces the following disadvantages, relative to ICF:

1. MCF requires more shielding masses, and will therefore probably offer less performance (the question being, just how much less performance).

2. Most MCF concepts rely on hybrid-plume technologies, in which additional expellant is added to the fusion products to increase mass flow rate and hence thrust, but these technologies will severely reduce the jet efficiency unless the added expellant can be thermodynamically mixed with the fusion products.

There is widespread misunderstanding about item 2, so perhaps an example will help clarify that added expellants must be thoroughly mixed (i.e., mixed to full temperature equilibrium). The jet efficiency is the ratio of the jet power to the input power, or one-half the square of the thrust divided by both the mass flow rate and the input power. If there are two distinct species involved, we obtain the following:

$$
\varepsilon_{j e t}=\frac{\left[\frac{d m_{1}}{d t} v_{1}+\frac{d m_{2}}{d t} v_{2}\right]^{2}}{\left[\frac{d m_{1}}{d t}+\frac{d m_{2}}{d t}\right]\left[\frac{\left.d m_{1} v_{1}{ }^{2}+\frac{d m_{2}}{d t} v_{2}{ }^{2}\right]}{d t}\right.}
$$

Inserting $10 \mathrm{~kg} / \mathrm{s}$ at $10^{3} \mathrm{~m} / \mathrm{s}$ into a stream of $10^{-3} \mathrm{~kg} / \mathrm{s}$ at $10^{6} \mathrm{~m} / \mathrm{s}$ yields a jet efficiency of only 0.012 or $1 \%$. Inserting $1 \mathrm{~kg} / \mathrm{s}$ at $10 \mathrm{~m} / \mathrm{s}$ into a stream of $10^{-3} \mathrm{~kg} / \mathrm{s}$ at $10^{5} \mathrm{~m} / \mathrm{s}$ yields a jet efficiency of only 0.0012 or $0.1 \%$. Thus, because it is usually a very difficult if not impossible job to thermalize added expellant into a hot fast-moving plasma stream in a magnetic environment, ${ }^{111}$ most MCF concepts employing hybrid plumes may not be practical because of the lowered jet efficiencies associated with the dual nature of the 
exhaust. Consequently, it is difficult if not impossible physically for a MCF concept to employ variable specific impulse. This is not to say it cannot be done, but only that it is difficult, and usually impossible because investigators have not realized that the jet efficiency is seriously degraded.

\section{XVI.2 Nuclear Electric Propulsion (NEP) Concepts}

Fission produces neutrons and gamma rays, and these particles are difficult to form directly into an exhaust having any significant mass-flow rate. If an indirect approach is considered whereby centimeters of matter are used to absorb the energy of these particles, then the exhaust temperature is usually limited to the melt temperature of the material used to stop the particles $(<1 \mathrm{eV})$. Fusion, on the other hand, has a charged plasma debris of temperature $\geq 1 \mathrm{keV}$, depending on the amount of extra mass (expellant) the fusion reactions must heat up. Thus, fission-powered engines can provide high power/mass ratios but only low exhaust velocities for significant mass flow rates, and nuclear-electric propulsion usually provides high exhaust velocities but only low power/mass ratios because of inefficient energy conversion — but fusion can theoretically provide both high power/mass ratios and high exhaust velocities. For single-stage self-contained spacecraft that must go roundtrip without using questionable maneuvers (e.g., air braking in the Martian atmosphere), VISTA performance (e.g., roundtrips to Mars in $\sim 5$ months) will necessarily be better than the performance for nuclear-thermal and nuclear-electric fission systems ( $\geq 2 / 3$ year) because the effective exhaust temperature (i.e., specific impulse) is roughly ten times larger for high mass flow rates. In fact, as seen from Fig. XI-3, VISTA performance is unsurpassed ( $<2 / 3$ year) for target gains $\geq 500$.

\section{XVI.3 Antimatter-Initiated Fission/Fusion}

Tom Nugent wrote a very nice report comparing VISTA with the so-called ion compressed antiproton-catalyzed nuclear (ICAN) propulsion system. ${ }^{112}$ Unfortunately, the formula he was given to indicate target gain as a function of driver energy is now known to be grossly in error. With a proper formula, the ICAN performance expectations do not compare favorably with that for VISTA.

In general, when antiprotons are used to initiate fusion through a fission sequence so that DT is heated to ignition temperature through the energy loss of the fission fragments, typically $10^{16}$ to $10^{17}$ antiprotons may be required per target. ${ }^{113}$ This is an excessive number of antiprotons-Fermi Lab can generate only 1 microgram $=6 \times 10^{17}$ antiprotons per year. If $10^{7}$ to $10^{8}$ targets are required for a mission (e.g., to Mars), it could take up to 10,000 years for Fermi Lab to generate enough antiprotons even if Fermi Lab's capacity were increased by two orders of magnitude!

For pure antimatter-matter annihilation, as opposed to antimatter-initiated fission/fusion, the generation, transfer, and storage of antimatter are important but not the only considerations. All pulsed propulsion methods are subject to three constraints if the rocket exhaust is formed by redirection of a plasma by a magnet: (1) the energy in the magnetic field must be at least five times the total energy in the plasma for one pulse, (2) the gyroradii of the individual particles must be considerably smaller than the dimensions of the apparatus, and (3) the dynamics (and primarily the time scale) of the redirection must be short enough to avoid significant Rayleigh-Taylor (interchange) instabilities. To assess 
the impact of these constraints for any concept, a detailed systems analysis is required, especially to determine the optimum extra mass (expellant) to be added per target to minimize trip time. Because antimatter drive can be tailored so as to have roughly the same energy yield per pulse as fusion schemes, and can have particulate gyroradii much smaller than typical magnets even for $\mathrm{MeV}$ energies, the real dynamic limitation for pure antimatter annihilation for space propulsion may arise from the systems analysis and not from the above constraints. In particular, antimatter-matter annihilation products (even with heavy-metal admixtures) can have $\mathrm{MeV}$ energies, which (depending on their type) can be orders of magnitude larger than that which will easily thermalize in expellant masses that might optimize the mission in terms of specific impulse and other parameters. The result is that significantly more antiprotons are needed per target than a first consideration might suggest. For example, for the Mars roundtrip reported in Table XV-2 with 3.6x10 engine pulses, we require $9 \mathrm{~kg}$ of antiprotons, which is three orders of magnitude larger than what we would expect from the numbers in the paragraph immediately above for antimatter-initiated fission/fusion. We therefore emphasize the importance of a detailed systems analysis for any antimatter concept.

\section{XVI.4 Orion-Type Pusher-Plate Concepts Using Nuclear Explosions}

It is not our intent here to analyze concepts employing thermonuclear explosions set off at a rate of up to $10 \mathrm{~Hz}$ behind a pusher plate in order to propel spacecraft forward, such as in the Orion ${ }^{114}$ proposal. Nevertheless, such concepts generally have two problems: 1) any significant vaporization of mass off the pusher plate will create a bimodal distribution of propellant velocities in the plume, which can seriously reduce the jet efficiency; and 2) large-enough explosions can cause material pressures exceeding the plastic-flow rates for typical structural materials (e.g., $\sim 2 \mathrm{kpsi}$ for $\mathrm{Al}$ ), so materials development could be a big factor. It is difficult to say more about these concepts because system studies are lacking.

\section{XVI.5 General Considerations}

It is common today for people to categorize concepts based on their total liftoff (wet) mass. After all, it would of course be more desirable to launch a so-called innovative confinement concept (ICC) system with a wet mass of 40 to 80 metric tons rather than launch VISTA with 6,000 metric tons, especially in terms of the launch costs (which, at about $\$ 10 \mathrm{k}$ per pound, would be over $\$ 130 \mathrm{~B}$ for VISTA). However, the ICCs are relatively new and in a state of infancy in terms of their development, so much physics and engineering information about them is simply unknown. This makes the ICCs high risk even though they may claim low mass and low cost. On the other hand, VISTA is basically a proven technology, and it permits safe interplanetary transport in regards to the zero-gee and cosmic-radiation hazards to the astronauts. There would hence appear to be a general tradeoff between low-mass high-risk systems and the high-mass low-risk systems. This is just an appearance, however, because the wet masses of the ICC systems will turn out to be much larger than proposed once more of the details of their systems have been considered.

For example, the cosmic-radiation threat in space arises from high-energy protons that interact in the shell and structure of any spacecraft, thereby forming a shower of highenergy pions and other particles that can cause leukemias and other illnesses in the crew, especially for exposures longer than one year. The mean free path $(\mathrm{mfp})$ of such strongly interacting hadrons (e.g., charged pions) is approximately $140 \mathrm{~g} / \mathrm{cm}^{2}$ in most spacecraft 
materials (i.e., $123 \mathrm{~g} / \mathrm{cm}^{2}$ in air, 140 in $\mathrm{Al}, 162$ in $\mathrm{Fe}, 215 \mathrm{in} \mathrm{Pb}$, etc.). Because the atmosphere is about $1000 \mathrm{~g} / \mathrm{cm}^{2}$ thick (i.e., 10 meters of water, or 8 pion $\mathrm{mfp}$ ), terrestrial humans near sea level experience no threat from these showers because the hadrons have died out leaving only muons that interact very weakly. But in space, it is nearly impossible physically to protect one person from this cosmic-radiation threat without providing a shield whose thickness is equivalent to at least 2 to 3 hadron $\mathrm{mfp}$ (more than this is of course desirable, but there is a tradeoff between better protection and lower shield mass). A thickness of 2 to 3 pion mfp corresponds to 1.0 to 1.6 meters of aluminum (or 38 to $57 \mathrm{~cm}$ of $\mathrm{Pb}$ )-----that is, at least 3 to $4 \mathrm{~m}$ water-equivalent in terms of the product of thickness and density (which is what determines the interaction probability).

Because the cosmic-radiation threat is isotropic and ever present (unlike a solar-flare threat corresponding to a timed event in a certain direction), the crew quarters must normally be surrounded by a shield for the crew to be protected from this threat.

Consequently, the portion of the shield for each person can be likened to a cylindrical section with a human-size (vertical) circumference of about $2 \pi \mathrm{m}=6 \mathrm{~m}$ and a (horizontal) width of at least $1 \mathrm{~m}$. As explained above, the thickness of this shield must be at least 3 to 4 $\mathrm{m}$ (water-equivalent) so it is at least 2 to 3 pion $\mathrm{mfp}$ thick. This amount of shielding is at least $(6 \mathrm{~m}) \times(1 \mathrm{~m}) \times(3$ to $4 \mathrm{~m}) \times\left(1\right.$ metric ton $\left./ \mathrm{m}^{3}\right)=18$ to 24 metric tons per person. This is one reason why VISTA includes a shield mass of nearly 200 metric tons, but VISTA is also large enough that the crew quarters can be located inside the propellant tanks, and this minimizes the shielding mass being discussed here. And of course, the crew shield is only one item on the mass-breakdown list-----we certainly have to include the engine, payload, fuel, structure, radiators, etc. We may also have to include other shielding to protect the crew from the fusion reactions, depending on the nature of the fusion engine and its proximity to the crew. So, it would seem as though the launch mass must necessarily be much larger than 100 metric tons for a multiple-person crew to be safe. Consequently, any fusion concept claiming a lift-off mass of less than 100 metric tons for a multiple-person crew is obviously forgetting some of the important details regarding crew safety!

There are also many general physics considerations that help us develop proper figures of merit for viable fusion-powered space-propulsion concepts. One such physics consideration is whether a concept can ever serve the propulsion community better than existing technologies (e.g., fission). Specifically, consider a known fission-propulsion system that is economically and environmentally viable for a certain mission. Then, if the ultimate long-range performance for a particular fusion concept cannot exceed that expected for the fission system, which is obviously based on a more mature technology than the fusion system, common sense tells us that we may not want to spend the time and resources required to develop that fusion system.

Consider, for example, a piloted roundtrip to Mars. A nuclear thermal rocket (or very advanced nuclear electric propulsion system) is expected to yield a roundtrip flight duration of about 1.0 to 1.5 years, so fusion, to be useful, must do "better" in order to justify the time and cost to develop the fusion systems. The usefulness of fusion is not merely to provide shorter flight durations, per se, but also to help the crew avoid (1) the physiological deterioration arising from zero-gee exposures lasting more than 100 days, and (2) the significant onset of leukemia and other cancers arising from exposures to the cosmic radiation exceeding one year. We might also consider the environmental advantages of fusion systems (e.g., less radioactivity hazard for material launched through the atmosphere). In short, there are many issues.

Nevertheless, one criterion for down-selection is whether a credible systems analysis (or even a basic physics assessment) can show that the concept could ever reach a level of performance better than what nuclear fission is expected to provide. This criterion can be made quantitative by requiring that the estimated flight duration be shorter than that 
expected for fission systems. Better yet, we can use the primary dynamical figure of merit for flight duration, namely, alpha $(\alpha)$, which we define in this document as the ratio of the dry mass to the jet power in $\mathrm{kg} / \mathrm{kW}$. For example, for a Mars roundtrip, computer calculations indicate that the $\alpha$ corresponding to a total flight duration of roughly one year is about 5 to $10 \mathrm{~kg} / \mathrm{kW}$ if all onboard systems (driver, power, radiator, etc. systems) are $100 \%$ efficient, or about 1 to $2 \mathrm{~kg} / \mathrm{kW}$ for systems with typical inefficiencies (i.e., the inefficiencies necessitate a smaller alpha to achieve the same performance). The alpha for known fission systems for a Mars roundtrip is thus of order unity, $\alpha_{\text {fission }}=O(1)$. Therefore, a goal-oriented criterion for a Mars roundtrip can be stated as requiring a candidate fusion concept to be modified or even eliminated (at some point) if a credible systems analysis shows that its flight system cannot achieve an alpha less than unity, $\alpha<\mathrm{O}(1)$. To support any fusion concept having $\alpha>>1$ would hence be merely of academic interest.

By following this approach further, we can examine the limitations set by certain physical constraints. Such constraints can force a minimum value for $\alpha$ that is larger than unity for certain concepts and hence affect their viability in a down-selection process. For example, an ICC system might be thought to have a dry mass of only tens of metric tons (i.e., between $10^{4}$ and $10^{5} \mathrm{~kg}$ ). One is therefore tempted to believe that getting $\alpha<1$ should be easy because the jet power need only exceed $10^{4}$ to $10^{5} \mathrm{~kW}$. However, getting $\alpha<\mathrm{O}(1)$ is not as easy as one might think, as the following arguments demonstrate.

A system's specific impulse $\mathrm{I}_{\mathrm{sp}}$ is defined by the magnitude of the thrust $\mathrm{F}$ projected along the rearward direction and the mass flow rate $(\mathrm{dm} / \mathrm{dt})$,

$$
g I_{s p}=\frac{|\vec{F}|}{(d m / d t)}
$$

where $g$ is $9.8 \mathrm{~m} / \mathrm{s}^{2}$. The jet power $\mathrm{P}_{\text {jet }}$ is then

$$
P_{j e t}=\frac{|\vec{F}|^{2}}{2(d m / d t)}=\left(\frac{1}{2}\right)(d m / d t)\left(g I_{s p}\right)^{2}
$$

The jet efficiency $\varepsilon_{\text {jet }}$ is defined as

$$
\varepsilon_{\text {jet }}=\frac{P_{\text {jet }}}{P_{\text {input }}}
$$

where $\mathrm{P}_{\text {input }}$ is the power inputted to the jet (not the power inputted to the engine). Through $\mathrm{P}_{\mathrm{jet},}$ the jet efficiency includes degradations in jet power arising from such things as nonmono-energetic exhaust velocity distributions, non-axial exhaust emissions requiring geometric projections of momenta on the thrust axis, non-mono-atomic-weight exhaust distributions, and coupling (drag) effects.

For fusion systems pulsed at a reprate $v \mathrm{~Hz}$ with driver energy $\mathrm{E}_{\mathrm{dr}}$ and fusion energy gain $\mathrm{G}$, the input power in Eq. (27) is

$$
P_{\text {input }}=v E_{d r}(G+1)
$$


where the "plus one" accounts for the driver energy itself. Using Eqs. (27) and (28) to obtain $\mathrm{P}_{\text {jet }}$ and equating the result with the value in Eq. (26), we find that the specific impulse is

$$
g I_{s p}=\sqrt{\frac{2 v E_{d r}(G+1) \varepsilon_{j e t}}{(d m / d t)}}
$$

This result demonstrates that the specific impulse is dependent on the jet efficiency. Even for a system with a mono-energetic axial exhaust velocity $\mathrm{v}_{\mathrm{ex}}$, the $\mathrm{I}_{\mathrm{sp}}$ is still dependent on the jet efficiency because the jet power is, from Eqs. (27) and (28),

$$
P_{j e t}=v E_{d r}(G+1) \varepsilon_{j e t}=\left(\frac{1}{2}\right)(d m / d t) v_{e x}^{2} \varepsilon_{j e t} \quad\left(\text { single } \mathrm{v}_{\mathrm{ex}}\right)
$$

so, making this substitution for the numerator in Eq. (29), we get

$$
g I_{s p}=v_{e x} \sqrt{\varepsilon_{j e t}} \quad\left(\text { single } \mathrm{v}_{\mathrm{ex}}\right)
$$

A concept's $I_{\text {sp }}$ should thus include the jet-efficiency factor. It is for this reason that we report specific impulse in this document in terms of Eq. (31), which we define to be the "effective specific impulse."

For the general case, the quantity $\alpha$ can be defined using Eqs. (26) and (29) as

$$
\alpha=\frac{m_{d r y}}{P_{j e t}}=\frac{m_{d r y}}{\frac{1}{2}\left(\frac{d m}{d t}\right)\left(g I_{s p}\right)^{2}}=\frac{m_{d r y}}{v E_{d r}(G+1) \varepsilon_{j e t}}
$$

where $E_{d r}$ is in $\mathrm{kJ}$ and $\mathrm{m}_{\mathrm{dry}}$ is in $\mathrm{kg}$. The criterion mentioned above requiring $\alpha$ to be less than of order $1 \mathrm{~kg} / \mathrm{kW}$ thus demands the fusion gain $\mathrm{G}$ to be larger than a minimum $\mathrm{G}_{\min }$ that can be established from Eq. (32):

$$
(G+1)>\left(G_{\min }+1\right)=\frac{m_{d r y}}{v E_{d r} \varepsilon_{j e t}}
$$

This is a key equation, because differing technologies place different restrictions on the maximum gain achievable.

At this point, we might be tempted to insert a dry mass of $10^{4}$ to $10^{5} \mathrm{~kg}$ for some ICC having (e.g.) a reprate of $30 \mathrm{~Hz}$, a driver energy of $10^{3} \mathrm{~kJ}$, and a jet efficiency of $40 \%$. We would then obtain a result for $\left(G_{\min }+1\right)$ of 0.8 to 8 , which is well within the capability of many ICC systems. However, this is an incorrect result-we cannot independently choose the above numbers because they violate another constraint. To see this, and correctly apply Eq. (33), we must first examine this other constraint.

Let $\mathrm{f}$ be the recycled power fraction. Obviously, we need $\mathrm{f}<<1$; for example, the power $\mathrm{fP}_{\text {jet }}$ recycled to operate a driver with efficiency $\varepsilon_{\mathrm{dr}}$ (as well as other equipment) must be rather small compared to the jet power itself. Considering a Mars trip and using $\alpha_{\text {fission }}=$ 1 in the first part of Eq. (32), we find that this constraint means the following for the fusion driver: 


$$
P_{d r}=\frac{v E_{d r}}{\varepsilon_{d r}} \ll P_{j e t}=\frac{m_{d r y}}{\alpha}>\frac{m_{d r y}}{1 k g / k W} \quad \text { (Mars trip) }
$$

Ignoring the power recycled for other equipment, we can calculate $\mathrm{f}$ by taking the ratio of the terms at the ends of Eq. (34) and obtain

$$
f=\frac{v E_{d r}}{\varepsilon_{d r} m_{d r y}}<<1 \quad \text { (Mars trip) }
$$

where again $\mathrm{E}_{\mathrm{dr}}$ is in $\mathrm{kJ}$ and $\mathrm{m}_{\mathrm{dry}}$ is in $\mathrm{kg}$, so Eq. (33) becomes

$$
(G+1)>\left(G_{\min }+1\right)=\frac{1}{f \varepsilon_{j e t} \varepsilon_{d r}}>>1 \quad \text { (Mars trip) }
$$

Jet efficiencies for fusion systems are typically about $1 / 3$ from geometric considerations alone (i.e., from projections of the exhaust velocities on the thrust axis). Driver efficiencies (at least for ICF systems) are near 10\% (but might be as much as $40 \%$ for some ICCs), and f should really be less than roughly a few percent. Therefore, according to Eq. (36), the fusion gain for any viable concept must be at least $\sim 250$ to just match the performance for fission systems. Computer calculations for VISTA agree with this rough assessment, and show that performance doesn't really shine in comparison with that for fission systems until G for VISTA is greater than about 500. In any case, for pulsed fusion systems where target gain is a well-defined quantity, we have

$$
G>\sim 250 \text { for Mars trips }
$$

This is an important result. For example, for magnetized target fusion (MTF) systems, $\mathrm{G}$ is expected to be at most about 70 because the burn is volumetrically ignited. Therefore, to be considered competitive (i.e., viable), a credible systems analysis for a flight system powered by MTF must demonstrate the required performance-the performance cannot be assumed. The reason for this is that, given everything else the same, MTF systems with $G<70$ cannot perform even as well as fission systems. This does not mean that research on MTF should stop, but it does mean that an added burden of proof exists for MTF systems. Such a consideration is over and above those relating to the infancy of MTF in terms of physics understanding and engineering development. VISTA, on the other hand, can have $G$ much above 250 if the advanced target designs can be developed, and VISTA is based on an ICF technology whose basic performance has already been proved. This discussion proves the necessity for credible systems analyses that include all relevant details. 


\section{CONCLUSIONS}

This study is significant for several reasons. It brought together a study team with a broad range of expertise to do a full systems analysis of a single-stage self-powered vehicle for piloted interplanetary transport. This analysis was comprehensive in comparison to mere conceptual studies using the rocket equation; we considered detailed thermal, electrical, and fusion-target systematics as well as waste-heat radiation and micrometeoroid shielding. The result was the VISTA conceptual design and documentation showing that VISTA is indeed a viable concept if target gains of at least 500 can be developed-no other technology, antimatter propulsion included, can then beat VISTA's performance within the solar system. In essence, VISTA brings the earlier configuration of Rod Hyde to the point of reality, and as such, became the first study of its kind for a realistic fusion-powered spacecraft.

In addition, this study identifies the critical issues and technology developments relating to VISTA systems, and estimates the time frame for the required R\&D. It identifies the risks for deployment of DT fusion fuels, specifically regarding tritium transport and neutron emissions, and explains how VISTA can use DD or $\mathrm{D}^{3} \mathrm{He}$ fuels if available. The VISTA study is also the first study showing how to deliver astronauts to their destinations within the interplanetary medium (specifically to Mars) in a functional state free of the deleterious physiological hazards from both zero gravity and cosmic-ray neutron-cascade showers. The VISTA concept even incorporates variable thrust (through variable rep rate) and variable specific impulse (through variable expellant mass per target). Payload masses can be hundreds of tons. Target gains $>1000$ permit exploration anywhere within the interplanetary environment and return in missions under 10 years, and to Mars and back in about 5 months. The VISTA concept thereby opens the solar system to safe piloted transport.

This study, however, is only the beginning. We have identified many areas that need clarification and / or further study to either improve VISTA's performance or increase confidence in the VISTA concept. We invite and encourage other investigators to expand on the notions introduced here. 


\title{
APPENDIX A: HIGH-PERFORMANCE SPACECRAFT SCALING
}

\author{
By John B. Trenholme
}

This section considers the constraints and scaling that apply to any form of highperformance space vehicle. By "high-performance," we mean spacecraft that can accelerate to extremely high velocities (hundreds or even thousands of kilometers per second) and cover solar-system distances in weeks or months. The requirement of high performance leads to a system that produces extremely high power per unit vehicle mass for a long period of time, and to a system that reaches high velocity by expelling a total mass considerably larger than the "dry" ship mass. As a consequence of the inevitable inefficiencies of any physical system, some of the engine power required to execute such a mission becomes waste heat absorbed by the spacecraft. This waste heat must be dissipated by radiation into space, and the combined requirements of high radiated power and large fuel mass dictate the overall configuration of the vehicle.

We will consider a simplified model that captures the scaling behavior of highperformance spacecraft without bogging us down in unimportant details. We suppose a mission in free space, and do not consider the effects of planetary or solar gravity. We ignore relativistic effects in both the vehicle mission and the propulsion system. These effects can be studied later, without significant change in the conclusions reached here.

Chemical energy is inadequate to propel the kinds of missions we are contemplating, because exhaust velocities comparable to the mission velocities are required (we shall see that the optimal exhaust velocity is about twice the average mission velocity). Chemical bonds cannot supply the energies per particle that are required to reach such velocities. The expulsion power therefore has to come from nuclear reactions such as fission or fusion, from matter-antimatter reactions, or from some external source (perhaps beamed power of some sort). We do not examine the details of the propulsion system, but instead consider the performance of any high-energy-per-exhaust-particle system. All that we require is that there be enough power available to push mass to the rear at very high velocity.

Some fuel has to be "burned" to supply exhaust power (except for beamed-power systems), but much of the "fuel" mass may well be non-energy-producing material, expelled for propulsion without itself producing power. As we shall see, it may be to our advantage to reduce the exhaust velocity in order to increase the thrust, by mixing in thermalized nonreactive exhaust mass (expellant) and decreasing the energy per particle. We will henceforth refer to the expelled mass as "fuel" even if much of it is inert expellant. The "fuel" used here is hence more properly termed "propellant." The parameters of the spacecraft are as follows:

The "dry" (no-fuel) ship mass is ' $s$ '. Some fraction of this is useful payload, while the rest is engine and auxiliary systems. Fuel mass is assumed to be carried without any tank mass - either the tanks are consumable as inert expulsion mass, or the fuel is solid and requires no tanks. Without tanks, we need not concern ourselves with multi-staged spacecraft.

The rocket force is constant at ' $\mathrm{f}$ '. Some improvement of mission performance is possible by using a variable-thrust engine, but we will ignore that possibility here. The acceleration of the ship is $\mathrm{g}=\mathrm{f} / \mathrm{m}$ while the engine is on, where ' $\mathrm{m}$ ' is the mass of the ship plus fuel at any instant.

The mean rearward component of exhaust expulsion velocity is constant at ' $\mathrm{e}$ '. There 
will inevitably be some distribution of speeds in the exhaust, and some distribution of directions. Perfection is achieved with no variation in either of these factors, and in that ideal case the mean rearward component is equal to the speed of any single exhaust particle.

The mission we consider consists of loading a large quantity of fuel at the origin. The ship starts its engine and accelerates steadily to high speed, burning or expelling more than half the fuel mass. The ship then turns off the engine and coasts at constant velocity for some period. It then reverses direction, restarts the engine, and decelerates until it arrives at the destination with zero velocity. We assume fuel is available at the destination, or that the ship will not be reused after the mission, and so we arrive with no fuel on board. This gives maximum performance. The cases of a roundtrip mission or a one-way non-stop mission are simple extensions, but we do not consider them here. Note that maximum acceleration occurs at the end of the mission with near-zero fuel, since we have assumed constant thrust and the accelerated mass is least at the end of the mission. The final acceleration is equal to $\mathrm{g}_{\mathrm{F}}=\mathrm{f} / \mathrm{s}$.

As mentioned above, the engine is assumed to be imperfect, in that it sprays its exhaust over some range of angles, and in that the exhaust products have a variety of speeds. Consider the expulsion of some small mass ' $\delta \mathrm{m}^{\prime}$ ' of exhaust during a short interval of time. We write the mean momentum of this mass element (in a frame moving with the ship) as $\left\langle\delta \mathrm{m} \cdot \phi \cdot \mathrm{s}_{\mathrm{E}}\right\rangle=\mathrm{m} \cdot \mathrm{e}$. Here ' $\mathrm{s}_{\mathrm{E}}$ ' is the speed of some portion of the exhaust, ' $\phi$ ' is the fraction of that portion's speed directed rearward, and <angle brackets $>$ indicate the mean of a quantity over its distribution. The mean force is then

$$
f=\frac{d(m e)}{d t}=-e \frac{d m}{d t}
$$

We have used the convention that a negative sign (mass loss) indicates a push on the ship as mass decreases. The rate of fuel mass loss is thus seen to be

$$
\frac{d m}{d t}=-\frac{f}{e}
$$

The power in the exhaust is the rate at which kinetic energy is added to the exhaust

$$
p_{E}=\frac{d}{d t} \frac{m\left\langle s_{E}^{2}\right\rangle}{2}=\frac{e^{2}}{2} \frac{\left\langle s_{E}^{2}\right\rangle}{e^{2}} \frac{d m}{d t}=\frac{\alpha e^{2}}{\beta^{2}} \frac{d m}{d t}=\frac{\alpha e f}{\beta^{2}}
$$

Here the factor ' $\alpha$ ' corrects for the difference between the mean of the square speed and the square of the mean speed (and is not to be confused with the $\alpha$ in $\mathrm{kg} / \mathrm{kW}$ in the main text of this report). For a single-speed exhaust, $\alpha$ has its lowest possible value of $1 / 2$, and any variation of speeds in the exhaust leads to an undesirable increase in ' $\alpha$ '. For a thermal distribution, ' $\alpha$ ' is more than twice its minimum value, and is equal to

$$
\alpha_{T H}=\frac{1}{2} \sqrt{\frac{3 \pi}{2}}=1.085402
$$


The factor ' $\beta$ ' corrects for the fact that not all the velocity that contributes to the kinetic energy is pointed backward to give useful thrust. The "backwardness" or collimation factor ' $\beta$ ' is defined as the mean ratio of force component backward, to total force without regard to direction. It is equal to the cosine of the mean angle away from directly rearward.

Note that we are adopting the convention that Greek letters are used to represent technology or engineering factors. The $p_{\mathrm{E}}$ equation shows that the velocity-force product 'e.f' is constant for a given exhaust power and speed-angle distribution:

$$
e f=\frac{p_{\mathrm{E}} \beta^{2}}{\alpha}
$$

This means that at constant power there is a tradeoff between velocity and thrust, with higher exhaust velocity leading to lower thrust, and vice versa. This tradeoff will turn out to be an important factor in maximizing mission performance, because the rocket power is limited by thermal dissipation in any physical system, and we wish to get the highest possible performance within a constant-power limit.

The specific energy $(\mathrm{J} / \mathrm{kg})$ in the expelled mass, produced by the engine, is equal to

$$
\frac{p_{E}}{\left(-\frac{d m}{d t}\right)}=\frac{\alpha e f}{\beta^{2}} \frac{e}{f}=\alpha\left(\frac{e}{\beta}\right)^{2}
$$

This quantity is a measure of our propulsion technology. Chemical engines are a poor choice for high performance precisely because they cannot put more energy into the exhaust than that available from the breaking of chemical bonds. The result is an exhaust velocity that is well below optimum for high-performance missions. Nuclear reactions can put some fraction of the fuel's rest mass energy into the exhaust. Antimatter reactions can, in principle, put an even larger fraction of the rest mass energy into the exhaust. No matter what technology is used, however, there will be an upper limit on the exhaust velocity ' $\mathrm{e}$ ' at or below the speed of light. Even if we could convert all the fuel mass to photons and somehow collimate them rearward, we shall see that this does not give the proper balance between exhaust velocity and thrust unless we intend to reach a velocity near light-speed. The price of light-speed exhaust is low thrust per unit engine power, and hence low performance for missions that do not approach light-speed (note that momentum is $\mathrm{hv} / \mathrm{c}$ for a photon, but at least $(2 \mathrm{mT})^{0.5} \approx \mathrm{T} /(0.5 \mathrm{v})$ for a particle of speed $\mathrm{v}$ and kinetic energy $\left.\mathrm{T}\right)$.

The most important limitation to performance is that the ship must absorb and dissipate some finite fraction of the rocket power ' $\mathrm{p}_{\mathrm{E}}$ '. Define ' $\gamma$ ' as the fraction of exhaust power absorbed by the ship, so that the power ' $p$ ' that the ship must dissipate is

$$
p=\gamma p_{E}=\frac{\alpha \gamma e f}{\beta^{2}}
$$

The absorbed-power fraction ' $\gamma$ ' is small if the ship is designed to intercept as little as possible of the drive power. The requirement to dissipate the absorbed power is what sets the performance limit of the spacecraft, since otherwise our undefined but arbitrarily powerful engine could increase power without limit to decrease mission time. Advanced engineering will be reflected in smaller values of ' $\alpha$ ' and ' $\gamma$,' and in larger values of ' $\beta$.' A fusion-driven ship (such as VISTA) will absorb heat by a number of routes, including: 
- particle impact due to particle leakage from the magnetic field

- radiation out of the fusion burn region and expelled exhaust

- eddy currents induced by the large magnetic field changes during each fusion microexplosion

- dissipation of vibrational energy caused by the impulsive nature of the drive

- inefficiency of the propulsion and other systems.

As we shall see, we need a refrigeration system to keep the crew and payload cool, and the ship must also dissipate the waste heat from the refrigerator.

As an example suppose that the exhaust velocity ' $\mathrm{e}$ ' is $1,000 \mathrm{~km} / \mathrm{s}$ or about $0.3 \%$ of light speed. The energy per particle in the exhaust is then about $5 \times 10^{-6}$ of its rest mass energy. Assume that the thrust force is 10,000 nt (or about 2,000 lb-f), that ' $\alpha$ ' is unity (thermal exhaust velocity distribution), that the fraction ' $\gamma$ ' of exhaust power absorbed is 0.1 , and that the collimation factor ' $\beta$ ' is 0.8 . Then the power ' $p$ ' that must be dissipated by the ship is

$$
p=\frac{\alpha \gamma e f}{\beta^{2}}=\frac{1.0 \cdot 0.1 \cdot 10^{6} \cdot 10^{4}}{0.8^{2}}=1.56 \times 10^{9} \mathrm{~W}
$$

This is $1.6 \mathrm{GW}-$ the power output of a large electric power plant. We must expel $\mathrm{f} / \mathrm{e}=10$ $\mathrm{g} / \mathrm{s}$ of mass to get this performance $(8640 \mathrm{~kg} /$ day). The e.f product is $10 \mathrm{GW}$.

The only presently known method of long-term, high-power dissipation in space is thermal radiation. If the radiators are black (the best case) and see only empty space, they radiate as $\sigma \theta^{4}$ when at temperature $\theta$ (recall we are using Greek letters for technology factors). Because of the $\theta^{4}$ scaling, the radiator size and mass increase rapidly as we go to lower radiating temperatures. We are strongly motivated to run at the highest possible radiator temperature if we want high performance. As a reference point, $\sigma \theta^{4}$ is about 300 $\mathrm{kW} / \mathrm{m}^{2}$ at the rather hot temperature of $1250 \mathrm{C}\left(1520^{\circ} \mathrm{K}\right.$, or yellow heat). To dissipate our $1.6 \mathrm{GW}$ reference case, we would need about 5,300 square meters of effective radiating area - for example, two sides of a thin square radiator over 50 meters on a side.

We shall return to the question of the radiators later, when we need to relate the ship's mass to its dissipated power. For the moment, we ignore the fact that there is a relation between ' $p$ ' and 's.'

Let us return to the mission parameters. We will review the standard equations of a rocket that expels a large fraction of its initial mass. Recall that the thrust force ' $\mathrm{f}$ ' is

$$
f=-\frac{d(m e)}{d t}=-e \frac{d m}{d t}
$$

and that the equation for the rate of change of total (ship plus fuel) mass ' $\mathrm{m}$ ' is

$$
\frac{d m}{d t}=-\frac{f}{e}
$$

When we integrate this latter equation in time, starting with an initial mass ' $i$ ' for the ship plus its fuel, we get the expected constant-rate mass decrease when thrust is on:

$$
m=i-\int_{0}^{t} \frac{f}{e} d t=i-\frac{f t}{e}
$$


Since we will end our trip when all the fuel is burned and mass equals ship mass ' $s$ ', the total time under power ' $u$ ' (acceleration plus deceleration) must be

$$
u=\frac{e(i-s)}{f}
$$

Let us introduce the ratio ' $\mathrm{R}$ ' of initial mass (ship plus fuel) to final mass (ship only)

$$
R=\frac{i}{S}
$$

The time under power is then given by

$$
u=\frac{e s(R-1)}{f}
$$

Recall we accelerate, coast, and then decelerate. If we introduce the ship-plus-fuel coast mass ' $c$ ' as an intermediate variable, the acceleration time ' $a$ ' is

$$
a=\frac{e(i-c)}{f}
$$

The deceleration time ' $\mathrm{d}$ ' is similarly seen to be

$$
d=\frac{e(c-s)}{f}
$$

By Newton's second law we have

$$
\frac{d \mathrm{v}}{d t}=\frac{f}{m}
$$

Combined with the $\mathrm{dm} / \mathrm{dt}$ equation, we have

$$
\frac{d v}{d m}=-\frac{e}{m}=-\frac{e}{\left(i-\frac{f t}{e}\right)}
$$

This can be integrated to give the well-known equation relating velocity change ' $\delta \mathrm{v}^{\prime}$ to initial ship-and-fuel mass ' $i$ ' divided by remaining ship-plus-fuel mass ' $m$ ' at any specific time

$$
\delta v=\int_{i}^{m}\left(\frac{-e}{m^{\prime}}\right) d m^{\prime}=e \ln \left(\frac{i}{m}\right)
$$


The total velocity change the ship is capable of making is given by

$$
\delta v_{M A X}=e \ln \left(\frac{i}{S}\right)=e \ln (R)
$$

The coast (turnover) velocity ' $\mathrm{v}_{\mathrm{c}}$ ' can be found in terms of the coast mass ' $\mathrm{c}$ ' as

$$
v_{c}=e \ln \left(\frac{i}{c}\right)
$$

We are assuming force-free space, so velocity is constant this value during coast. This is also the maximum velocity reached during the mission.

After coasting for some period of time, we decelerate down to velocity zero at final ship-only mass 's,' so we have

$$
0=v-\int_{c}^{s}\left(\frac{-e}{m}\right) d m=e \ln \left(\frac{i}{c}\right)-e \ln \left(\frac{c}{s}\right)=e \ln \left(\frac{i s}{c^{2}}\right)
$$

This zero-end-velocity condition is satisfied when $\mathrm{c}^{2}=\mathrm{i} \cdot \mathrm{s}$. This lets us solve for the coast mass as the geometric mean of the initial (fuel plus ship) and final (ship only) masses

$$
c=\sqrt{i s}
$$

We substitute this into the equation for coast velocity ' $\mathrm{v}_{\mathrm{c}}$ ' and show the well-known fact that the coast velocity is just half the maximum velocity change

$$
v_{c}=e \ln \left(\frac{i}{\sqrt{i S}}\right)=e \ln \left(\sqrt{\frac{i}{s}}\right)=e \ln (\sqrt{R})=\frac{e \ln (R)}{2}=\frac{\delta v_{M A X}}{2}
$$

This motivates us to introduce the ratio ' $r$ ' of coast fuel-plus-ship mass to bare-ship mass (and also initial to coast fuel-plus-ship mass) as a useful intermediate variable

$$
r=\frac{c}{s}=\frac{i}{c}=\sqrt{\frac{i}{s}}=\sqrt{R} \quad r^{2}=R
$$

We can write a number of trip parameters in terms of ' $r$ ' and get: 


$$
\begin{aligned}
& \text { coast mass: } \quad c=s r=s(r-1) \\
& \text { coast fuel: } \quad=s r^{2}=s R \\
& \text { initial mass: } \quad i==s\left(r^{2}-1\right)=s(R-1) \\
& \text { initial fuel: } \quad \mathrm{v}_{\mathrm{C}}=e \ln (r) \\
& \text { coast velocity: } \\
& \text { initial deceleration: } \quad \mathrm{g}_{\mathrm{D}}=\frac{f}{r s} \\
& \text { final deceleration: } \quad \mathrm{g}_{\mathrm{F}}=\frac{f}{s} \\
& \text { deceleration time: } \quad d=\frac{e s(r-1)}{f} \\
& \text { initial acceleration: } \quad \mathrm{g}_{\mathrm{A}}=\frac{f}{r^{2} s}=\frac{f}{R s} \\
& \text { acceleration time: } \quad a=\frac{e s r(r-1)}{f}=r d \\
& \text { power }- \text { on time: } \quad u=a+d=\frac{e s\left(r^{2}-1\right)}{f}=\frac{e s(R-1)}{f}
\end{aligned}
$$

Note that ' $r$ ' is the ratio of acceleration to deceleration times

$$
r=\frac{a}{d}
$$

This means that acceleration must always last longer than deceleration-much longer if the initial mass is mostly fuel.

The minimum value of ' $r$ ' is unity. If ' $r$ ' is 10 then the initial configuration is 99 parts fuel to 1 part ship (by mass) so large values of ' $r$ ' correspond to a tiny ship attached to a huge fuel mass. The factor 'es / $f$ ' that appears several times above is the time it takes the ship to dispose of an amount of fuel mass equal to its own mass.

We now want to find the distances covered during the acceleration and deceleration phases, so that we can find the coast distance and time. The acceleration distance ' $y$ ' is found by integrating

$$
\frac{d x}{d m}=\frac{d x / d t}{d m / d t}=\frac{-e \mathrm{~V}}{f}
$$

We integrate from initial mass to coast mass to get

$$
y=\int_{s r^{2}}^{s r}\left[\frac{-e^{2} \ln \left(\frac{s r}{m}\right)}{f} \mid d m=\frac{e^{2} s r[r-1-\ln (r)]}{f}\right.
$$


The deceleration distance is most easily found by viewing from a frame moving at the coast velocity ' $\mathrm{v}_{\mathrm{C}^{\prime}}$ ' so the coasting ship is at relative rest when deceleration starts. Then deceleration moves the ship by the moving-frame distance ' $n$,' given by

$$
n=\int_{s r}^{s}\left|\frac{\left.-e^{2} \ln \left(\frac{s r}{m}\right)\right]}{f}\right| d m=-\frac{e^{2} s[r-1-\ln (r)]}{f}
$$

Note that ' $n$ ' is just $y / r$. The total distance ' $z$ ' moved during deceleration is the motion of the frame plus the relative motion, so that

$$
z=\mathrm{v}_{\mathrm{c}} d+n=\frac{e^{2} s[1+r \ln (r)-r]}{f}
$$

The total distance moved under power is the sum of the acceleration and deceleration distances

$$
y+z=\frac{e^{2} s(r-1)^{2}}{f}
$$

We make note here of the wonderful cancellation of the ' $r \cdot \ln (r)$ ' terms.

If we don't coast at all, but immediately turn over and begin decelerating at the end of the acceleration period, the total trip distance ' $x$ ' is minimal and equal to the powered distance $y+z$, and so we have

$$
x_{\min }=\frac{e^{2} s(r-1)^{2}}{f}
$$

We can solve this equation for ' $r$ ' to find

$$
r_{M A X}=1+\sqrt{\frac{x f}{e^{2} s}}
$$

This is the largest sensible ' $r$ ' value for this distance and these ship parameters. The amount of fuel corresponding to ' $\mathrm{r}_{\text {MAX }}$ ' is $\mathrm{s} \cdot\left(\mathrm{r}_{\max }{ }^{2}-1\right)$. Loading on more fuel than this amount makes it impossible to burn all the fuel during the trip, even if power is on continuously, and so leads to lower average acceleration and a longer trip time. If we put on less fuel than this amount, then we must coast for some of the trip. The coast velocity (for infinitesimal coast duration) corresponding to the largest sensible fuel load is

We now determine the total trip duration including a coast period of finite duration. The

$$
v_{\text {MAX }}=e \ln \left(1+\sqrt{\frac{x f}{e^{2} s}}\right)
$$

coast time is the coast distance ' $\mathrm{x}-(\mathrm{y}+\mathrm{z})$ ' divided by the coast velocity ' $v$,' so the coast time is 


$$
\frac{x-\frac{e^{2} s(r-1)^{2}}{f}}{e \ln (r)}
$$

The total trip time ' $\mathrm{t}$ ' is the sum of the power-on and coast times

$$
t=\frac{e s\left(r^{2}-1\right)}{f}+\frac{x-\frac{e^{2} s(r-1)^{2}}{f}}{e \ln (r)}=\frac{e s}{f}\left|\frac{\left[\frac{x f}{e^{2} s}-(r-1)^{2}\right.}{\ln (r)}+r^{2}-1\right|
$$

The trip time is large for values of ' $r$ ' close to unity, and for very large values of ' $r$,' and there is a minimum of ' $t$ ' with respect to ' $r$ ' between these two limits. Thus there is a fuel load value that minimizes trip time. We would like to solve this transcendental equation for the ' $r$ ' value that leads to minimum trip time. No analytic solution is possible, but we can find the minimum numerically and approximate the resulting ' $r$ ' values. During this process, it is useful to use normalized units. Define the normalized trip distance ' $j$ ' as

$$
j=\frac{x f}{e^{2} s}
$$

Define the normalized trip time ' $h$ ' as

$$
h=\frac{t f}{e s}
$$

The normalized trip-time equation is, in terms of ' $r$ ', ' $\mathrm{j}$ ' and ' $\mathrm{h}$ '

$$
h=\frac{j-(r-1)^{2}}{\ln (r)}+r^{2}-1
$$

For future reference, the fraction of the total trip time spent accelerating is

$$
\frac{t_{A}}{t}=\frac{r(r-1)}{\frac{j-(r-1)^{2}}{\ln (r)}+r^{2}-1}
$$

The fraction of the total trip time spent coasting is

$$
\frac{t_{C}}{t}=1-\frac{r^{2}-1}{\frac{j-(r-1)^{2}}{\ln (r)}+r^{2}-1}=\frac{j-(r-1)^{2}}{j-(r-1)^{2}+\left(r^{2}-1\right) \ln (r)}
$$

The fraction of the total trip time spent decelerating is 


$$
\frac{t_{D}}{t}=\frac{r-1}{\frac{j-(r-1)^{2}}{\ln (r)}+r^{2}-1}
$$

For these equations to apply, ' $r$ ' must be less than its maximum sensible value

$$
r \leq r_{\text {MAX }}=1+\sqrt{j}
$$

Define the ' $r$ ' value that minimizes the trip time as ' $\mathrm{r}_{\mathrm{OPT}}$.' It is straightforward to show that the limits for small and large ' $\mathrm{j}$ ' are

$$
\begin{aligned}
r_{\text {opt }}=1+\sqrt{j}=r_{M A X} & h_{\text {opt }}=2 \sqrt{j} & j \rightarrow 0 \\
r_{\text {opt }} \ln \left(r_{\text {opt }}\right)=\sqrt{\frac{j}{2}} & & j \rightarrow \infty
\end{aligned}
$$

We can solve the latter equation in terms of the Lambert " $w$ " function that obeys

$$
w(x) e^{w(x)}=x \quad \text { or } \quad w(x)+\ln (w(x))=\ln (x)
$$

We find that for very large ' $j$ ' we have

$$
r_{\text {opt }}=\frac{\sqrt{\frac{j}{2}}}{w\left(\sqrt{\frac{j}{2}}\right)} \quad j \rightarrow \infty
$$

For large $\mathrm{x}, \mathrm{w}(\mathrm{x})$ is approximated by

$$
w(x) \rightarrow \ln (x)-\ln [\ln (x)]+\ldots
$$

Therefore for very large ' $j$ ' we have

$$
r_{o p t} \rightarrow \frac{\sqrt{\frac{j}{2}}}{\ln \left(\sqrt{\frac{j}{2}}\right)} \quad j \rightarrow \infty
$$

This large-j asymptotic value is reached very slowly, because of the slowly-changing $\ln (\ln (\mathrm{x}))$ and higher terms in $\mathrm{w}(\mathrm{x})$. Fortunately, as we shall see, we are not interested in large values of ' $\mathrm{j}$.'

We can find the values of ' $r$ ' that minimize ' $h$ ' for various ' $j$ ' values by numerical minimization. Here are some representative values (see below for ' $r_{\text {approx }}$ ') 


\begin{tabular}{|c|c|c|c|c|c|c|}
\hline $\mathrm{j}$ & 0.001 & 0.01 & 0.1 & 1 & 10 & 100 \\
\hline $\mathrm{r}_{\mathrm{opt}}$ & 1.03114 & 1.09548 & 1.27858 & 1.74646 & 2.83640 & 5.32036 \\
\hline $\mathrm{h}_{\text {opt }}$ & 0.064238 & 0.209765 & 0.725889 & 2.84425 & 13.4024 & 75.9646 \\
\hline $\mathrm{r}_{\max }$ & 1.03162 & 1.1 & 1.31623 & 2 & 4.16228 & 11 \\
\hline $\mathrm{r}_{\text {approx }}$ & 1.03119 & 1.09591 & 1.28072 & 1.74799 & 2.81863 & 5.30578 \\
\hline
\end{tabular}

We see that the minimum-time fuel ratio ' $r$ ' is always less than the largest sensible value, which means that the minimum-time missions always involve a coast period. Loading on more fuel to keep the engine on longer is counterproductive, because the increased mass leads to smaller accelerations and a longer trip time.

By fitting the time-minimizing values of ' $r$ ' as a function of ' $j$ ' using a form that has the asymptotic behaviors given above, we can get a numerical approximation to ' $\mathrm{r}_{\text {opt }}$ ' with reasonable accuracy (max. relative error $\pm 1 \%$ )

$$
r_{\text {approx }}=1+\frac{\sqrt{j}}{\left\{1+\sqrt{2} \ln \left[\frac{1+0.51192 \sqrt{j}}{1+2.06834 \ln (1+0.096966 \sqrt{j}}\right]\right\}}
$$

If greater accuracy is desired, we can use Newton-Raphson iteration of the equation that sets the derivative of ' $h$ ' with respect to ' $r$ ' equal to zero. By starting with the above approximation, three iterations give 16-digit accuracy for any value of ' $\mathrm{j}$.' The iteration is

$$
r_{N+1}=\frac{2 r_{N}^{2} \ln \left(r_{N}\right)\left[\ln \left(r_{N}\right)+1\right]-\left(r_{N}-1\right)^{2}+j}{2 \ln \left(r_{N}\right)\left[2 r_{N} \ln \left(r_{N}\right)+1\right]}
$$

We will probably not use these minimum-time fuel-loading values in actual missions. Near the optimum, the trip time increases only quadratically as we decrease the fuel loading. It saves us fuel linearly to use less than the optimum, but only takes more time as the square of the deviation from optimum. Therefore trips can be done with less fuel by using ' $r$ ' values below the minimum-time values, without paying much penalty in increased trip time. The exact balance depends on the relative values of fuel and time to the person designing the mission.

Although we can use the above approximation to find the best loading for any specific power-limited values of ' $e^{\prime}$ and ' $f$,' it turns out that we can improve mission performance (decrease mission time) by adjusting ' $\mathrm{e}$ ' and ' $\mathrm{f}$ ' while keeping their product constant to maintain constant engine power. Remarkably, there is a global minimum time and corresponding globally optimum fuel-loading ratio. If at all possible, we should use this fuel loading for any mission distance, and adjust ' $\mathrm{e}$ ' and ' $\mathrm{f}$ ' to minimize trip time. For large distances, we may be constrained by the maximum ' $\mathrm{e}$ ' our propulsion system is capable of providing, but when this is not the case, every minimum-time mission starts with the same fuel load ratio. Every minimum-time mission accelerates, coasts, and decelerates for the same fractions of the total trip time. Every minimum-time mission has the same ratio of peak velocity to mean velocity.

To demonstrate this extraordinary new result, we need to solve the trip equation to find the minimum trip time. It is better to solve the transcendental trip-time equation by proceeding "backwards," by assuming a value for ' $r$ ' and evaluating ' $j$ ' and ' $h$ ' from that value. This gives us a parametric representation for the normalized time and distance, with the parameter being 'r.' 
Given any selected value for ' $r$ ', we can find the value of ' $j$ ' for which that ' $r$ ' gives minimum trip time by taking the derivative of the equation for ' $h$ ' with respect to ' $r$ ' and setting it to zero. Values of ' $j$ ' for which the selected ' $r$ ' value we started with does not minimize trip time will have non-zero slope at the selected ' $r$ ' value, and will be rejected because their minimum-time ' $j$ ' values are not equal to the one we started with. We find that

$$
j_{o p t}(r)=2 r \ln (r)[r \ln (r)-r+1]+(r-1)^{2}
$$

This function varies as $(r-1)^{2}$ for ' $r$ ' near unity, and as $2[r \cdot \ln (r)]^{2}$ for very large ' $r$ '. As expected, these asymptotic $\mathrm{j}(\mathrm{r})$ behaviors correspond to the asymptotic $\mathrm{r}(\mathrm{j})$ behaviors seen above.

By substituting the expression for ' $\mathrm{j}_{\text {opt }}$ ' into the equation for ' $\mathrm{h}$ ' we find the minimum normalized trip time to be

$$
h_{\text {opt }}=2 r^{2} \ln (r)-(r-1)^{2}
$$

We now have a pair of minimum-time ' $j$ ' and ' $h$ ' values parametrically in terms of ' $r$.' We will next replace the normalized distance and time by the un-normalized physical values and determine the minimum possible trip time. Before we do that, however, we need to relate the ship's mass to the power it dissipates.

Recall that we are assuming that power absorbed by the ship is dissipated by radiation into empty space, by radiating surfaces with black-body efficiency at absolute temperature $\theta$. The effective radiator area (the area that looks at empty space and not other radiators or the Sun) must be large enough to radiate all the absorbed power, so the area must be

$$
A_{R A D}=\frac{p}{\sigma \theta^{4}}
$$

The radiators must not see portions of themselves if radiative efficiency is to be maximized. The only geometry that avoids self-irradiation is a near-planar or flat-plate configuration. We conclude that any high-performance spacecraft looks like an incandescent planar object. The radiators must be positioned with the sun and any nearby warm body in their plane, so that the radiator sees only black space. We probably also want to keep ships well separated, to avoid mutual overheating.

For maximum ship performance, we want minimum mass in the radiators. Let's assume them to be two thin, roughly square fins of side length 'L.' The total radiating area (two fins, with two sides each) is $4 \mathrm{~L}^{2}$. The fin side length is then

$$
L=\frac{1}{2 \theta^{2}} \sqrt{\frac{p}{\sigma}}
$$


We want to minimize the fin mass. We'll make the fins as thin as possible, with the lowest possible density, but it is impossible to make them arbitrarily thin compared to their length and width. If ' $\varepsilon$ ' is the ratio of thickness to side (low ' $\varepsilon$ ' means advanced technology) then the volume is $2 \mathrm{~L}^{3} \varepsilon$. The structure might be made of a space-filling mass of small struts (probably pressurized with gas to increase buckling resistance) and wires, with highperformance heat pipes carrying the heat from the engine area to the surfaces. Even with most of the volume empty, we need some fraction of solid density to do the structural and heat transport work. The formula for radiator mass we will use is

$$
m_{R A D}=\frac{\rho \varepsilon}{4 \theta^{6}}\left(\frac{p}{\sigma}\right)^{3 / 2}
$$

where ' $\rho$ ' is the average density of the radiator structure. Advanced technology allows us to decrease radiator mass by building low- $\rho$, low- $\varepsilon$ radiators running at high temperatures, but the square-cube power scaling stays the same. This scaling reflects the basic fact that radiation goes as the square of size, but mass goes as the cube. The dramatic sixth-power decrease of mass with radiator temperature is a strong motivation to run at very high temperatures. The crew and cargo spaces (and perhaps portions of the engine) will have to be refrigerated, but the rest of the ship will run hot. At the low acceleration values we will be using, the ship can be of extremely flimsy construction - for highest performance, we want a ship made of "incandescent tissue paper."

Since we want minimum ship mass per unit exhaust power, we should make the rest of the ship's mass small compared to m $\mathrm{RAD}$. For simplicity, we assume from here on that the entire ship mass ' $\mathrm{s}$ ' scales as $\mathrm{m}_{\mathrm{RAD}}$ above. This is the best case-fixed masses for the engine and payload will make things worse. Adding a fixed mass to the radiator mass is straightforward, but adds unimportant complication. We now have a ship mass of

$$
s=\frac{\rho \varepsilon}{4 \theta^{6}}\left(\frac{p}{\sigma}\right)^{3 / 2}
$$

Let us return to our example ship with $1.6 \mathrm{GW}$ dissipation, and assume a thinness ratio $\varepsilon$ of 0.02 and a density $\rho$ of $100 \mathrm{~kg} / \mathrm{m}^{3}$ (about $1 / 30$ of solid density, depending on the materials). The radiator (and ship) mass is then

$$
s=\frac{100 \cdot 0.02}{4 \cdot 1520^{6}}\left(\frac{1.6 \times 10^{9}}{5.67051 \times 10^{-8}}\right)^{3 / 2}=1.92 \times 10^{5} \mathrm{~kg}=192 \text { tonnes }
$$

With the assumed thrust of 10,000 nt, the final (end-of-trip) acceleration is

$$
g_{F}=\frac{1 \times 10^{4} \mathrm{nt}}{1.92 \times 10^{5} \mathrm{~kg}}=0.052 \frac{\mathrm{m}}{\mathrm{s}^{2}} \cong 0.005 \mathrm{~g}
$$

We now return to the determination of the global optimum fuel mass ratio. We will eliminate the thrust force ' $\mathrm{f}$ ' from the equations. To do so, we first consider the equation for 'j' 


$$
j=\frac{x f}{e^{2} s}
$$

We substitute the radiator-mass-only value for the ship mass ' $s$ ' and write the exhaust velocity ' $\mathrm{e}$ ' in terms of the thrust force ' $f$ ' using the power-limit equation. The result is

$$
j=\frac{4 x f^{3} \sigma^{3 / 2} \theta^{6} \alpha^{2} \gamma^{2}}{p^{7 / 2} \beta^{4} \rho \varepsilon}
$$

We can solve this for ' $\mathrm{f}$ ' to give

$$
f=\frac{p^{7 / 6} \beta^{4 / 3} \rho^{1 / 3} \varepsilon^{1 / 3} j^{1 / 3}}{4^{1 / 3} x^{1 / 3} \sigma^{1 / 2} \theta^{2} \alpha^{2 / 3} \gamma^{2 / 3}}
$$

Next we use the normalization equation for ' $h$ '

$$
h=\frac{t f}{e s}
$$

Substituting the same values for ' $s$ ' and 'e' that we used in the normalization equation for ' $\mathrm{j}$,' we get

This gives us another equation for ' $\mathrm{f}$ '

$$
h=\frac{4 t f^{2} \sigma^{3 / 2} \theta^{6} \alpha \gamma}{p^{5 / 2} \beta^{2} \rho \varepsilon}
$$

$$
f=\frac{p^{5 / 4} \beta \rho^{1 / 2} \varepsilon^{1 / 2} h^{1 / 2}}{2 t^{1 / 2} \sigma^{3 / 4} \theta^{3} \alpha^{1 / 2} \gamma^{1 / 2}}
$$

We can now eliminate ' $f$ ' by equating this equation to the one we got from the ' $j$ ' normalization:

$$
f=\frac{p^{7 / 6} \beta^{4 / 3} \rho^{1 / 3} \varepsilon^{1 / 3} j^{1 / 3}}{4^{1 / 3} x^{1 / 3} \sigma^{1 / 2} \theta^{2} \alpha^{2 / 3} \gamma^{2 / 3}}=\frac{p^{5 / 4} \beta \rho^{1 / 2} \varepsilon^{1 / 2} h^{1 / 2}}{2 t^{1 / 2} \sigma^{3 / 4} \theta^{3} \alpha^{1 / 2} \gamma^{1 / 2}}
$$

We now solve for the trip time ' $t$ ' in terms of the trip distance ' $x$, ' the dissipated power ' $p$,' and technology factors, times a function of the coast fuel mass ratio ' $r$.' We find:

$$
t=\frac{x^{2 / 3} p^{1 / 6}}{2^{2 / 3} \sqrt{\sigma \theta^{4}}}\left(\frac{\alpha \varepsilon \gamma \rho}{\beta^{2}}\right)^{1 / 3} b(r)
$$

where 


$$
b(r)=\frac{h_{o p t}(r)}{\left[j_{o p t}(r)\right]^{2 / 3}}=\frac{2 r^{2} \ln (r)-(r-1)^{2}}{\left[2 r \ln (r)\{r \ln (r)-r+1\}+(r-1)^{2}\right]^{2 / 3}}
$$

We are using the least-trip-time values of ' $h$ ' and ' $j$ ' in the function $b(r)$ to assure that we are always considering an optimum trip. Given this choice, we can still vary ' $r$ ' to change the trip time. It turns out that there is a minimum value of the trip time at one "magic" value of ' $r$.' This particular ' $r$ ' gives the least possible trip time, assuming we can vary ' $\mathrm{e}^{\prime}$ and ' $\mathrm{f}$ ' to their optimal values (shown below). The "magic" value comes at the minimum of $b(r)$ with respect to its argument:

$$
0=\frac{d b(r)}{d r}=\frac{2}{3} \frac{[2 r \ln (r)+1]\left\{2 \ln (r)\left[r^{2} \ln (r)-(r-1)(2 r+1)\right]+3(r-1)^{2}\right\}}{\left\{2 r \ln (r)[r \ln (r)-r+1]+(r-1)^{2}\right\}^{5 / 3}}
$$

The first factor in the numerator is positive in the interval $1<\mathrm{r}<\infty$, and so the "magic" $\mathrm{r}$ value solves the equation

$$
2 \ln (r)\left[r^{2} \ln (r)-(r-1)(2 r+1)\right]+3(r-1)^{2}=0 \quad 1<r<x
$$

There is only one solution, which must be found numerically

$$
r_{M}=2.102526489
$$

The resulting minimum value of $b(r)$ is

$$
b_{M}=2.794210589
$$

We can now find many quantities of interest at this "magic" value of 'r.' The initial mass ratio is

$$
R_{M}=4.420617637
$$

The trip time is

$$
t_{M}=1.760242369 \frac{x^{2 / 3} p^{1 / 6}}{\sqrt{\sigma \theta^{4}}}\left(\frac{\alpha \varepsilon \gamma \rho}{\beta^{2}}\right)^{1 / 3}
$$

Note that the trip time increases if the ship's power dissipation is increased. This counterintuitive result is a consequence of the fact that the radiator mass required to dissipate the absorbed power rises faster than the increase of performance with increased available power. This behavior is a consequence of the square-cube scaling law for thermal radiators. It is better to build lightweight ships of lower power if we want the highest-performance missions. Of course, there is often a minimum size that any power system can be built at, but we should build at or near that minimum size if at all possible. Two smaller ships can always complete a high-performance mission quicker than one larger ship, and so forth. 
The exhaust velocity is

$$
e_{M}=1.146696057 \frac{x^{1 / 3} \sqrt{\sigma \theta^{4}}}{p^{1 / 6}}\left(\frac{\beta^{2}}{\alpha \varepsilon \gamma \rho}\right)^{1 / 3}
$$

The thrust force is

$$
f_{M}=0.8720706711 \frac{p^{7 / 6}}{x^{1 / 3} \sqrt{\sigma \theta^{4}}}\left(\frac{\beta^{4} \varepsilon \rho}{\alpha^{2} \gamma^{2}}\right)^{1 / 3}
$$

The coast velocity is

$$
v_{C M}=0.8521553771 \frac{x^{1 / 3} \sqrt{\sigma \theta^{4}}}{p^{1 / 6}}\left(\frac{\beta^{2}}{\alpha \varepsilon \gamma \rho}\right)^{1 / 3}
$$

Note that the scaling of $\mathrm{v}_{\mathrm{CM}}$ is the same as $\mathrm{e}_{\mathrm{M}}$, and only the coefficient is different. The optimal coast velocity is always $74 \%$ of the optimal exhaust velocity.

The average velocity is

$$
v_{A M}=\frac{x}{t_{M}}=0.5681035847 \frac{x^{1 / 3} \sqrt{\sigma \theta^{4}}}{p^{1 / 6}}\left(\frac{\beta^{2}}{\alpha \varepsilon \gamma \rho}\right)^{1 / 3}
$$

Note that the ratio of coast to average velocity is exactly $3 / 2$. This is true only for the "magic" fuel mass ratio - the velocity ratio is 2 for very low initial fuel loading and decreases steadily as ' $r$ ' increases. The algebra to show that the "magic" value is $3 / 2$ is straightforward but tedious, and so we do not show it here. The optimal average velocity is always $49.5 \%$ of the optimal exhaust velocity.

The accelerations at the beginning of acceleration, at the beginning of deceleration, and at the finish of the trip are

$$
\begin{aligned}
& g_{A M}=0.7890939618 \frac{\sigma \theta^{4}}{x^{1 / 3} p^{1 / 3}}\left(\frac{\beta^{2}}{\alpha \varepsilon \gamma \rho}\right)^{2 / 3} \\
& g_{D M}=1.659090957 \frac{\sigma \theta^{4}}{x^{1 / 3} p^{1 / 3}}\left(\frac{\beta^{2}}{\alpha \varepsilon \gamma \rho}\right)^{2 / 3} \\
& g_{F M}=3.488282685 \frac{\sigma \theta^{4}}{x^{1 / 3} p^{1 / 3}}\left(\frac{\beta^{2}}{\alpha \varepsilon \gamma \rho}\right)^{2 / 3}
\end{aligned}
$$

The normalized values of the trip distance and time are 


$$
\begin{aligned}
& j_{M}=2 r_{M} \ln \left(r_{M}\right)\left[r_{M} \ln \left(r_{M}\right)-r_{M}+1\right]+\left(r_{M}-1\right)^{2}=2.652864291 \\
& h_{M}=2 r_{M}^{2} \ln \left(r_{M}\right)-\left(r_{M}-1\right)^{2}=5.354708373
\end{aligned}
$$

The fractions of the trip time spent accelerating, coasting and decelerating are

$$
\begin{aligned}
\frac{\mathrm{t}_{A M}}{t} & =\frac{r_{M}\left(r_{M}-1\right)}{h_{M}}=0.4329070767 \\
\frac{\mathrm{t}_{C M}}{t} & =1-\frac{r_{M}^{2}-1}{h_{M}}=0.3611944108 \\
\frac{\mathrm{t}_{D M}}{t} & =\frac{r_{M}-1}{h_{M}}=0.2058985125
\end{aligned}
$$

The fractions of the trip distance spent accelerating, coasting and decelerating are

$$
\begin{aligned}
& \frac{x_{A M}}{x}=\frac{r_{M}\left[r_{M}-1-\ln \left(r_{M}\right)\right]}{j_{M}}=0.2848318408 \\
& \frac{x_{C M}}{x}=1-\frac{\left(r_{M}-1\right)^{2}}{j_{M}}=0.5417916162 \\
& \frac{x_{D M}}{x}=\frac{r_{M} \ln \left(r_{M}\right)-r_{M}+1}{j_{M}}=0.1733765430
\end{aligned}
$$

Let us return to our example ship, which we recall has e $=1,000 \mathrm{~km} / \mathrm{s}, \mathrm{f}=10,000 \mathrm{nt}, \alpha=1, \gamma$ $=0.1, \beta=0.8, p=1.6 \mathrm{GW}, \theta=1520 \mathrm{~K}, \rho=100 \mathrm{~kg} / \mathrm{m}^{3,} \varepsilon=0.02$ and $\mathrm{s}=192,000 \mathrm{~kg}$. What mission is this ship best suited for? The best distance, where the parameters are "magic," is given by

$$
x_{M}=\frac{j_{M} e^{2} s}{f}=\frac{2.65286 \cdot\left(10^{6} \mathrm{~m} / \mathrm{s}\right)^{2} \cdot 1.92 \times 10^{5} \mathrm{~kg}}{1 \times 10^{4} \mathrm{nt}} \cong 5.1 \times 10^{13} \mathrm{~m}
$$

This is about 340 A.U. The time is given by $h_{m} \cdot e \cdot s / f$, but we use the full formula

$$
t_{M}=1.760242369 \frac{\left(5.1 \times 10^{13} \mathrm{~m}\right)^{2 / 3}\left(1.6 \times 10^{9} \mathrm{~W}\right)^{1 / 6}}{\sqrt{5.67051 \times 10^{-8} \cdot 1520^{4}}}\left(\frac{1 \cdot 0.02 \cdot 0.1 \cdot 100}{0.8^{2}}\right)^{1 / 3} \cong 10^{8} \mathrm{~s} \cong 3.2 \text { years }
$$

The mission starts by loading on a fuel mass equal to

$$
s\left(r_{M}{ }^{2}-1\right)=192,000 \mathrm{~kg}(4.4206-1) \cong 660,000 \mathrm{~kg}
$$

The ship then starts out with an initial acceleration of about $0.001 \mathrm{~g}$. Applied over a period of 500 days, and increasing as fuel mass is expelled, the acceleration builds up a velocity of

$$
v_{C M}=0.8522 \frac{\left(5.1 \times 10^{13} \mathrm{~m}\right)^{1 / 3} \sqrt{\sigma(1520 \mathrm{~K})^{4}}}{\left(1.6 \times 10^{9} \mathrm{~W}\right)^{1 / 6}}\left(\frac{0.8^{2}}{1 \cdot 0.02 \cdot 0.1 \cdot 100 \mathrm{~kg} / \mathrm{m}^{3}}\right)^{1 / 3} \cong 750 \mathrm{~km} / \mathrm{s}
$$


This is a very high velocity by the standards of today's chemical or ion rockets, but it is "only" $0.25 \%$ of light-speed. The ship will need extremely effective micrometeoroid defenses at this coast velocity.

The ship coasts for 420 days, and then turns over and begins decelerating at $0.002 \mathrm{~g}$. It has already covered $83 \%$ of the total distance. After 240 days of deceleration, it comes to a stop with a final deceleration of $0.005 \mathrm{~g}$. The ship is now about ten times as far from its starting point as the radius of the solar system. Getting this far in a little over three years is indeed a high-performance mission.

How do ship designs vary with the mission distance? Realizing that "small is beautiful" if we want high performance, let's consider ships that dissipate only $100 \mathrm{MW}$ with other technology factors set to the values we have been using:

- Exhaust velocity distribution factor $\alpha=1$ (thermal distribution)

- $\quad$ Exhaust angular distribution factor $\beta=0.8$

- Exhaust power absorption fraction $g=0.1$ (exhaust useful power $1 \mathrm{GW}$ )

- Radiator fin temperature $\theta=1520^{\circ} \mathrm{K}$

- Radiator fin thickness-to-length ratio $\varepsilon=0.02$

- Radiator fin average density $\rho=100 \mathrm{~kg} / \mathrm{m}^{3}$

With these values, the radiator (and ship) mass is

$$
s=\frac{100 \cdot 0.02}{4 \cdot 1520^{6}}\left(\frac{10^{8}}{5.67051 \times 10^{-8}}\right)^{3 / 2}=3002.45 \mathrm{~kg} \cong 3 \text { tonnes }
$$

It will be a considerable challenge to build a $1 \mathrm{GW}$ engine that has a mass small compared to 3 tons, but we will assume it can be done. The payload also has to be small compared to 3 tons. The ship designs will be optimized for a variety of distances from 1 A.U. to 10,000 A.U., giving the following results:

\begin{tabular}{|c|c|c|c|c|c|}
\hline $\begin{array}{c}\text { Distance } \\
(\text { A.U. })\end{array}$ & $\begin{array}{c}\text { time } \\
\text { (one way) }\end{array}$ & $\begin{array}{c}\text { exhaust vel. } \\
(\mathrm{km} / \mathrm{s})\end{array}$ & $\begin{array}{c}\text { thrust } \\
(\mathrm{nt})\end{array}$ & $\begin{array}{c}\text { coast vel. } \\
(\mathrm{km} / \mathrm{s})\end{array}$ & $\begin{array}{c}\text { max. accel. } \\
(\mathrm{g})\end{array}$ \\
\hline 1 & 15.67 days & 222.9 & 2645 & 165.6 & 0.08984 \\
\hline 10 & 72.77 days & 480.2 & 1228 & 356.9 & 0.04170 \\
\hline 100 & 337.8 days & 1035 & 569.9 & 768.9 & 0.01936 \\
\hline 1000 & 4.293 years & 2229 & 264.5 & 1656 & 0.008984 \\
\hline 10000 & 19.93 years & 4802 & 122.8 & 3569 & 0.004170 \\
\hline 270000 & 179.3 years & 14410 & 40.93 & 10710 & 0.00139 \\
\hline
\end{tabular}

The last row corresponds to a mission to Alpha Centauri, and requires us to put almost $0.2 \%$ of their rest mass energy into the exhaust particles. These designs are audacious, but in return for design audacity, they give extremely high performance. They also give us a good feel for the maximum performance we might achieve with advanced nuclear-energy propulsion systems. Improving on the performance in the table will require us to build 
very small ships, run at extremely high radiator temperatures, or create designs that intercept and dissipate very small percentages of the engine power. The largest leverage is in radiator temperature, since trip time goes inversely with the square of this quantity, but running at $1500{ }^{\circ} \mathrm{K}$ is already a very difficult feat. We also see that any high-performance design requires high exhaust velocity, since optimal coast velocity is $74 \%$ of exhaust velocity. The performance drop-off for lower exhaust velocity is not abrupt, however, and there is some flexibility in the choice of this quantity. 
C. Orth, UCRL-LR-110500

\section{ACKNOWLEDGMENTS}

The work at LLNL was performed under the auspices of the U.S. Department of Energy by the University of California, Lawrence Livermore National Laboratory, under contract number W-7405-Eng-48. We thank the 1987 systems-study team consisting of Gail Klein and Joel Sercel of JPL, Nathan Hoffman and Kathy Murray of Rockwell/Rocketdyne/ETEC, and Franklin Chang-Diaz of NASA/JSC, because this document would never have existed without their help. We thank Bill Hogan and Max Tabak of LLNL, and Tien-Fang (Ted) Yang of MIT for helpful suggestions and comments during the early phases of the systems analysis. We thank Hoppy Price of JPL for drawing the VISTA layout, and Stan Krauthamer of JPL for investigating advanced powerconditioning systems. We thank Carl Sauer and Andrey Sergeyevsky of JPL for running the NASTRAN code for Mars missions. We thank Katie Barhydt, Alok Chatterjee, and Jayant Sharma of JPL for helpful suggestions. We thank Tom Trelenberg for investigating tritium hazards, and for computer calculations relating to alpha curves. We thank John Trenholme for writing Appendix A, and Ralph Moir for critically reading the manuscript.

\section{REFERENCES}

${ }^{1}$ R. A. Hyde, "A Laser Fusion Rocket for Interplanetary Propulsion," UCRL-88857, University of California, Livermore, CA (1983), and the 34th International Astronautical Federation, held in Budapest, Hungary, 10-17 October 1983.

${ }^{2}$ Sally K. Ride, "Leadership and America's Future in Space," NASA, Report to the Administrator (August 1987).

${ }^{3}$ Sally K. Ride, ibid.

${ }^{4}$ K. Boyer and J. D. Balcomb, "System studies of fusion powered propulsion systems," AIAA Paper No. 71-636 (June 1971).

5 “Manned Mars Missions: A Working Group Report," ed. by Michael B. Duke and Paul W. Keaton, NASA Report M001 (May 1986); and Nate Hoffman, ETEC, private communication (blood problems in zero gravity).

${ }^{6}$ Ibid.; and Nate Hoffman, ETEC, private communication (leukemias).

${ }^{7}$ Jeffery Kahn, “Cosmic Ray Questions," LBL Research Review, Vol. 16, No. 3, p. 2 (Fall/Winter 1991).

${ }^{8}$ C. Nance, "Nuclear pulse propulsion," IEEE Trans. on Nuclear Sci. NS-12, No. 1, (1965), 177-182; and F. J. Dyson, "Death of a project," Science, 149 (1965), 141-144.

${ }^{9}$ R. Hyde, L. Wood, and J. Nuckolls, "Prospects for rocket propulsion with laser-induced fusion microexplosions," AIAA Paper No. 72-1063 (December 1972).

${ }^{10}$ R. Forward, "Mars-Earth Rapid Interplanetary Tether Transport (MERITT) System: I. Initial Feasibility Analysis," 35 ${ }^{\text {th }}$ AIAA/ASME/SAE/ ASEE Joint Propulsion Conference, 20-24 June 1999, Los Angeles, CA, Paper AIAA 99-2151.

${ }^{11}$ J. D. Balcomb, et al., "Nuclear pulsed space propulsion systems," LA-4541-MS, Los Alamos Scientific Laboratory, Los Alamos, NM (November 1970).

${ }^{12}$ E.g., Bill Wagstaff, "A Spaceship Named Orion," Air \& Space, Smithsonian Institute (Oct./Nov. 1988).

${ }^{13}$ R. Hyde, L. Wood, and J. Nuckolls, "Prospects for rocket propulsion with laser-induced fusion microexplosions," AIAA Paper No. 72-1063 (December 1972).

${ }^{14} \mathrm{~F}$. Winterberg, "Rocket propulsion by thermonuclear micro-bombs ignited with intense relativistic electron beams," Raumfahrtforschung 15 (1971), 208-217; and Proceedings of the International School of Physics "Enrico Fermi," Physics of High Energy Density (NY: Academic Press, 1971), pp. 395-396.

${ }^{15}$ A. R. Martin, ed., "Project Daedalus: The Final Report on the B.I.S. Starship Study," J. British 
Interplanetary Society, Supplement (1978).

${ }^{16}$ R. A. Hyde, "A Laser Fusion Rocket for Interplanetary Propulsion," UCRL-88857, University of California, Livermore, CA (1983), and the 34th International Astronautical Federation, held in Budapest, Hungary, 10-17 October 1983.

${ }^{17}$ B. G. Cour-Palais, "Meteoroid Environment Model -1969 (Near Earth to Lunar Surface)," NASA SP 8013, 1969.

${ }^{18}$ Norman R. Schulze, Fusion Energy for Space Missions in the 21st Century, NASA Technical Memorandum 4298, p. 7-22 (August 1991).

${ }^{19}$ Norman R. Schulze, ibid., p. 7-24.

${ }^{20}$ Norman R. Schulze, ibid., p. 7-24.

${ }^{21}$ J. F. Santarius, "Magnetic Fusion for Space Propulsion," Fusion Technology, Vol. 21, p. 1794 (May 1992).

${ }^{22}$ J. F. Santarius, ibid.

${ }^{23}$ Norman K. Schulze, Fusion Energy for Space Missions in the 21st Century: Executive Summary, NASA Technical Memorandum 4297 (Aug. 1991).

${ }^{24}$ Max Tabak, J. Hammer, M. Glinsky, W. Kruer, S. Wilks, J. Woodworth, E. M. Campbell, M. Perry, and R. Mason, "Ignition and High Gain with Ultra-Powerful Lasers," Physics of Plasmas, Vol. 1 \# 5, pp. 1626-1634 (1994); and Max Tabak, private communication (1998).

${ }^{25}$ R. A. Sacks and D. H. Darling, "Direct Drive Cryogenic Capsules Employing DT Wetted Foam," UCRL94381, University of California, Livermore, CA (April 1986), and "Direct Drive Cryogenic ICF Capsules Employing D-T Wetted Foam," Nuclear Fusion, 27, \#3 (1987) 447-452.

${ }^{26}$ R. A. Sacks and D. H. Darling, ibid.

${ }^{27}$ Sümer Sahin, Haci Mehmet Sahin, and Adnan Sozen,"Evaluation of the Neutron and Gamma-ray Heating in the Radiation Shielding and Magnet Coils of the VISTA Spacecraft," Fusion Technology, 33 (July 1998) 418-434; and Sümer Sahin, Haci Mehmet Sahin, and Eyüp öztürk, "Reduced Radiation Shielding for the Magnet Coils of the VISTA Spacecraft," Ninth International Conference on Emerging Nuclear Energy Systems (ICENES'98), Tel-Aviv, Israel, June 28 - July 2, 1998, 1 (1998) 312-319; and Sümer Sahin and Haci Mehmet Sahin, "Radiation shielding mass saving for the magnet coils of the VISTA spacecraft," Annals of Nuclear Energy, 26 (1999) 509-521; and Sümer Sahin and Haci Mehmet Sahin, "Optimization of the radiation shielding mass for the magnet coils of the VISTA spacecraft," Annals of Nuclear Energy, 28 (2001) 1413-1429; and Sümer Sahin, Haci Mehmet Sahin, and Abdulmuttalip Sahinaslan, "Reduced Shielding Mass for the VISTA Spacecraft," Arabian Journal for Science and Engineering, 27 (2002) 187-196.

${ }^{28}$ Charles D. Hendricks, "Automated Laser Fusion Target Production Concept," UCRL-80209, University of California, Livermore, CA (4 October 1977); C. D. Hendricks and W. L. Johnson, "Power Plant Production of Inertial Confinement Fusion Targets," UCRL-52539, University of California, Livermore, CA (15 February 1979); C. D. Hendricks and W. L. Johnson, "Electrostatic Levitation, Control, and Transport in High-Rate Low-Cost Production of Inertial Confinement Fusion Targets," UCRL-82752, University of California, Livermore, CA (25 May 1979); Wayne L. Johnson and Charles D. Hendricks, "Electrostatic Levitation and Transport of Laser Fusion Targets," UCRL-83981, University of California, Livermore, CA (22 May 1980); J. W. Sherohman and C. D. Hendricks, "A Target Factory in Perspective," UCRL-83470, University of California, Livermore, CA (11 April 1980); and J. W. Sherohman and W. R. Meier, "A Parametric Study of a Target Factory for Laser Fusion," UCRL-84264, University of California, Livermore, CA (8 October 1980).

${ }^{29}$ R. W. Petzoldt, "IFE Target Injection and Tracking Experiment," Fusion Technology, 34 (1998) 831-839; and R. W. Petzoldt, R. W. Moir, "Target Injection and Tracking for Inertial Fusion Energy," University of California, Lawrence Livermore National Laboratory, Report UCRL-JC-122017 and International Symposium on Heavy Ion Inertial Fusion, Princeton, NJ (Sept. 6-9, 1995); also see M. J. Monsler, "Laser Fusion: An Assessment of Target Injection, Tracking, and Beam Pointing," UCRL-80563, University of California, Livermore, CA (5 June 1978), and CONF-780508, Procs. of the 3rd Topical Meeting on the Technology of Controlled Nuclear Fusion, 9-11 May 1978, Santa Fe, NM, Vol. 2, p. 1186.

${ }^{30}$ John H. Pitts, "Heat-Transfer Limitations on Targets Used in ICF Reaction Chambers," UCRL-85975, University of California, Livermore, CA (12 October 1981), and IEEE Pub. No. 81CH1715-2 NPS, 9th Symposium on Engineering Problems of Fusion Research, 26-29 October 1981, Chicago, IL, Vol. I, p. 676.

${ }^{31}$ R. W. Petzoldt, "Membrane Support for Accelerated Fuel Capsules for Inertial Fusion Energy," Fusion Technology, 30 (1996) 73-82. 
${ }^{32}$ R. W. Petzoldt and R. W. Moir, "Membrane Support of Accelerated Fuel Capsules for Inertial Fusion Energy," Fusion Technology, 30 (Sept. 1996) 73-82.

${ }^{33}$ John H. Pitts, ibid.

${ }^{34}$ M. J. Monsler, "Laser Fusion: An Assessment of Target Injection, Tracking, and Beam Pointing," UCRL80563, University of California, Livermore, CA (5 June 1978), and CONF-780508, Procs. of the 3rd Topical Meeting on the Technology of Controlled Nuclear Fusion, 9-11 May 1978, Santa Fe, NM, Vol. 2, p. 1186.

${ }^{35}$ M. D. Rosen, J. D. Lindl, and A. R. Thiessen, "Simple Models of High-Gain Targets--Comparisons and Generalizations," UCRL- 50021-83, University of California, Livermore, CA (June 1984), pp. 3-5 to 3-9. ${ }^{36}$ M. D. Rosen, ibid.

${ }^{37}$ J. D. Lindl, R. O. Bangerter, J. W-K. Mark, and Yu-Li Pan, "Review of Target Studies for Heavy Ion Fusion," UCRL-94382, University of California, Livermore, CA (24 October 1986) and Procs. of the International Symposium on Heavy Ion Fusion, Washington D.C. (Fig. 3).

${ }^{38}$ John Lindl, Physics of Plasmas, Vol. 2, No. 11 (1995), p. 3969, Eq. (100).

${ }^{39}$ Max Tabak, private communication, July 1998.

${ }^{40}$ Max Tabak, private communication, July 1998.

${ }^{41}$ M. Tabak, "What is the Role of Tritium-Poor Fuels in ICF?", Nuclear Fusion, 36, No. 2 (1966), p. 147.

${ }^{42}$ M. Tabak, Nuclear Fusion, ibid.

${ }^{43}$ Energy from Inertial Fusion (Vienna: International Atomic Energy Agency, 1995), p. 430.

${ }^{44}$ Sümer Sahin, et al., ibid.

${ }^{45}$ R. O. Bangerter, Lawrence Livermore National Laboratory, private communication (1986).

${ }^{46}$ J. Swingle, Lawrence Livermore National Laboratory, private communication (1986).

${ }^{47}$ D. Harris, Los Alamos National Laboratory, private communication (1986).

${ }^{48}$ J. H. Nuckolls, Lawrence Livermore National Laboratory, private communication (1987).

${ }^{49}$ C. D. Orth, S. A. Payne, and W. F. Krupke, "A Diode Pumped Solid State Laser Driver for Inertial Fusion Energy," Nuclear Fusion, 36, \#1 (1996) 75-116.

${ }^{50}$ C. D. Marshall, L. K. Smith, R. J. Beach, M. A. Emanuel, K. I. Schaffers, and S. A. Payne, "Diode pumped ytterbium-doped $\mathrm{Yb}: \mathrm{Sr}_{5}\left(\mathrm{PO}_{4}\right)_{3} \mathrm{~F}$ laser performance," IEEE J. Quantum Electronics, 32, \#4 (1996) 650 (and ibid., 1995 ASSL Conference Proceedings).

${ }^{51}$ C. D. Marshall, L. K. Smith, S. Sutton, M. A. Emanuel, K. I. Schaffers, S. Mills, S. A. Payne, W. F. Krupke, and B. H. T. Chai, "Diode-pumped gas-cooled-slab laser performance," OSA Trends in Optics and Photonics on ADVANCED SOLID STATE LASERS, 1, Ed. S. A. Payne and C. R. Pollock, Topical Meeting January 31 - February 2, 1996, San Francisco, CA, Optical Society of America (1996) 208-212.

${ }^{52}$ C. Orth, R. Beach, C. Bibeau, E. Honea, K. Jancaitis, J. Lawson, C. Marshall, R. Sacks, K. Schaffers, J. Skidmore, S. Sutton, "Design modeling of the 100-J diode-pumped solid-state laser for Project Mercury," SPIE Proceedings, Solid State Lasers VII, 23-30 January 1998, San Jose, CA, 3265, 114-129.

${ }^{53}$ C. D. Orth, S. A. Payne, and W. F. Krupke, ibid.

${ }^{54}$ Charles D. Orth, Joshua E. Rothenberg, Stephen A. Payne, Howard T. Powell, "Progress at LLNL Toward DPSSL-Driven Inertial Fusion Energy," Current Trends in International Fusion Research-Proceedings of the Third Symposium Edited by E. Panarella. NRC Research Press, National Research Council of Canada, Ottawa, ON K1A OR6 Canada (1999).

${ }^{55}$ C. Orth, R. Beach, C. Bibeau, E. Honea, K. Jancaitis, J. Lawson, C. Marshall, R. Sacks, K. Schaffers, J. Skidmore, S. Sutton, "Design modeling of the 100-J diode-pumped solid-state laser for Project Mercury," SPIE Proceedings, Solid State Lasers VII, 23-30 January 1998, San Jose, CA, 3265, 114-129.

${ }^{56}$ C. D. Marshall, L. K. Smith, R. J. Beach, M. A. Emanuel, K. I. Schaffers, and S. A. Payne, “Diode pumped ytterbium-doped $\mathrm{Yb}: \mathrm{Sr}_{5}\left(\mathrm{PO}_{4}\right)_{3} \mathrm{~F}$ laser performance," IEEE J. Quantum Electronics, 32, \#4 (1996) 650 (and ibid., 1995 ASSL Conference Proceedings).

${ }^{57}$ Eric C. Honea, Jay A. Skidmore, Barry L. Freitas, Everett Utterback, and Mark A. Emanuel, “Modeling chirp and sag effects in high-peak laser-diode-bar pump sources for solid-state lasers," ADVANCED SOLID STATE LASERS (2-4 Feb. 1998, Coeur d'Alene, Idaho), OSA Trends in Optics and Photonics, 19 (1998) 326-332.

${ }^{58}$ R. A. Hyde, "A Laser Fusion Rocket for Interplanetary Propulsion," UCRL-88857, University of California, Livermore, CA (1983), and the 34th International Astronautical Federation, held in Budapest, Hungary, 10-17 October 1983.

${ }^{59}$ D. Milam, Lawrence Livermore National Laboratory, private communication (July 1986). 
${ }^{60}$ D. Milam, ibid.

${ }^{61}$ D. Milam, ibid.

${ }^{62}$ Robert Bieri, "Grazing Incidence Metal Mirrors as a Solution to the Problem of Neutron Damage to Final Optics for a Laser Driver," University of California, Lawrence Livermore National Laboratory memo ICFR-LLNL-89-7 (April 4, 1989); W. R. Meier, R. L. Bieri, et al., "OSIRIS and SOMBRERO Inertial Confinement Fusion Power Plant Designs, Volume 2, Designs, Assessments, and Comparisons," W. J. Schafer Associates Report WJSA-92-01, DOE/ER/54100-1 (March 1992) esp. pp. 3-142 to 3-144; and "Inertial Fusion Energy Reactor Design Studies, PROMETHEUS-L, PROMETHEUS-H, Final Report," McDonnell Douglas Aerospace Report MDC 92E0008, DOE/ER-54101 (March 1992) esp. pp. 4-85 to 4-86 and 6.5.1-46 to 6.5.1-62.

${ }^{63}$ R. W. Moir, "Grazing incidence liquid metal mirrors (GILMM) as the final optics for laser inertial fusion energy power plants," University of California, Lawrence Livermore National Laboratory Report UCRLID-134612; and R. W. Moir, "Grazing incidence liquid metal mirrors (GILMM) for radiation hardened final optics for laser inertial fusion energy power plants," University of California, Lawrence Livermore National Laboratory Report UCRL-JC-132875 and the Fifth International Symposium on Fusion Nuclear Technology (ISFNT-5), Rome, Italy (September 19-24, 1999).

${ }^{64}$ R. A. Hyde, "A Laser Fusion Rocket for Interplanetary Propulsion," UCRL-88857, University of California, Livermore, CA (1983), and the 34th International Astronautical Federation, held in Budapest, Hungary, 10-17 October 1983.

${ }^{65}$ R. A. Hyde, ibid.

${ }^{66}$ R. A. Hyde, ibid.

${ }^{67}$ E.g., M. K. Wu, et al., "Superconductivity at $93 \mathrm{~K}$ in a New Mixed-Phase Y-Ba-Cu-O Compound System at Ambient Pressure," Phys. Rev. Lett. 58, No. 9 (2 March 1987) 908 .

${ }^{68}$ R. A. Hyde, "A Laser Fusion Rocket for Interplanetary Propulsion," UCRL-88857, University of California, Livermore, CA (1983), and the 34th International Astronautical Federation, held in Budapest, Hungary, 10-17 October 1983.

${ }^{69}$ Sümer Sahin, et al., ibid.

${ }^{70}$ Yu. P. Zakharov, A. G. Ponomarenko, H. Nakashima, Y. Nagamine, S. A. Nikitin, J. Wolowski, E. Woryna, "Direct Energy Conversion of Inertial Confinement Fusion and Experiments with Laser-Produced Plasma in Magnetic Fields," Ninth International Conference on Emerging Nuclear Energy Systems (ICENES'98), Tel-Aviv, Israel, June 28 - July 2, 1 (1998 ) 384-391 (esp. p. 388-389).

${ }^{71}$ F. R. Chang and J. L. Fisher, "A Supersonic Gas Target for a Bundle Divertor Plasma," Nuclear Fusion 22, No. 8, (1982) 1003; and F. R. Chang, W. A. Krueger, and T. F. Yang, "Numerical Modeling of the Hybrid Plume Plasma Rocket," AIAA/DGLR/JSASS 18th International Propulsion Conference (30 Sept. 1985).

${ }^{72}$ R. A. Hyde, ibid.

${ }^{73}$ R. A. Hyde, ibid.

${ }^{74}$ R. A. Hyde, ibid.

${ }^{75}$ A. R. Martin, ed., "Project Daedalus: The Final Report on the B.I.S. Starship Study," J. British Interplanetary Society, Supplement (1978).

${ }^{76}$ R. A. Hyde, ibid.

${ }^{77}$ W. R. Meier, et al., "Inertial Fusion Power for Space Applications," UCRL-94138, University of California, Livermore, CA (1986).

${ }^{78}$ C. Moyers, "ALKACYCL, A Basic Computer Program for the Analysis of Alkali Metal Rankine Power Cycles," Report ORNL/TM 9693, Oak Ridge National Laboratory, Oak Ridge, TN (1985).

${ }^{79}$ G. L. Yoder and R. L. Graves, "Analysis of Alkali Liquid Metal Rankine Space Power Systems," Transactions of the Second Symposium on Space Nuclear Power Systems, held in Albuquerque, NM, January 1985.

${ }^{80}$ J. H. Pitts and M. H. L. Jester, "Rankine Cycle Systems Studies for Nuclear Space Power," Proceedings of the Third IECEC Conference, Boulder, CO, 1968; and J. H. Pitts and C. E. Walter, "Conceptual Design of a $10 \mathrm{MW}_{\mathrm{e}}$ Nuclear Rankine System for Space Power," J. Spacecraft 7 (3) 259-265.

${ }^{81}$ J. Sercel and S. Krauthamer, "Multi-Megawatt Nuclear Electric Propulsion; First-Order System Design and Performance Evaluation," Report AIAA-86-1202, Proceedings AIAA Space Systems Technology Conference, held in San Diego, CA, 9-12 June 1986.

${ }^{82}$ J. H. Pitts and C. E. Walter, "Conceptual Design of a $10 \mathrm{MW}_{\mathrm{e}}$ Nuclear Rankine System for Space Power," J. 
Spacecraft 7 (3) 259-265.

${ }^{83}$ R. A. Hyde, ibid.

${ }^{84}$ R. A. Hyde, ibid.

${ }^{85}$ R. A. Hyde, ibid.

${ }^{86}$ Private communication, Hoppy Price, JPL (1987).

${ }^{87}$ H. L. Kelley and C. R. Phillips, "Review of Tritium Monitoring Devices", Tritium, Messenger Graphics, Phoenix, Arizona and Las Vagas, Nevada, pp. 133 (1973).

${ }^{88}$ H. Gote Ostlund, "Tritium in the Atmosphere and Ocean", Tritium,-Messenger Graphics, Phoenix, Arizona and Las Vagas, Nevada, pp. 385 (1973).

${ }^{89}$ K. Y. Wong, T. A. Khan, and F. Guglieimi, Canadian Tritium Experience, Ontario Hydro, pp 96 (1984).

${ }^{90}$ Y. M. Shtukkenberg, "Physical Properties of Isotopes of Hydrogen", Tritium Oxide, Atomizdat, Moscow, pp. 11 (1968).

${ }^{91}$ Private communication, Sandra Brereton, Hazards Control Dept., LLNL (7 Nov. 1991).

${ }^{92}$ T. E. McKone and W. E. Kastenberg, "Calculation of Radiological Dose due to Release of Tritium Oxides from Controlled Thermonuclear Reactors", Nuclear Technology, Vol. 40, pp. 174 (Sept. 1978).

${ }^{93}$ T. E. McKone and W. E. Kastenberg, "Calculation of Radiological Dose due to Release of Tritium Oxides from Controlled Thermonuclear Reactors", Nuclear Technology, Vol. 40. pp. 174, (Sept. 1978).

${ }^{94}$ J. R. Johnson, E. S. Lamothe, J. S. Jackson, and R. G. C. McElroy, "Metabolism and Dosimetry of Tritium from Tritium Gas Contaminated Surfaces", Fusion Technology, Vol.14, pp. 1147 (Sept. 1988).

${ }^{95}$ Private communication, Jim Ellis, ARAC, LLNL (15 Nov. 1991).

${ }^{96}$ Private communication, Hans Kruger, D-Division, LLNL (21 Nov. 1991).

${ }^{97}$ Harold Johnston, "Evaluation of Excess Carbon 14 and Strontium 90 Data for Suitability to Test TwoDimensional Stratospheric Models", Journal of Geophysical Research, Vol. 94, pp. 18492 (20 Dec. 1989).

${ }^{98}$ Private communication, Jim Ellis, ARAC, LLNL (15 Nov. 1991).

${ }^{99}$ Private communication, Jim Ellis, ARAC, LLNL (15 Nov. 1991).

${ }^{100}$ HOT SPOT PC Health Physics Codes, S. G. Homann, UCRL - MA - 106315 (1992).

${ }^{101}$ Brown, Logram, and Spenser, "Field Studies of HT Behavior in the Environment: Dispersion and Oxidation in the Atmosphere", Fusion Technology, Vol.14, pp. 1165 (Sept. 1988).

${ }^{102}$ Environmental Protection Agency, Manual of Protective Action Guidelines and Protective Actions for Nuclear Incidents, EPA-520 / 1 - 75 - 001, 1975 (revised 1980).

${ }^{103}$ Private communication, John Nasstrom, ARAC, LLNL (4 Dec. 1991).

${ }^{104}$ Carl Sauer, Andrey Sergeyevsky, and Joel Sercel, Jet Propulsion Laboratory, private communication (1987).

${ }^{105}$ C. D. Orth, W. J. Hogan, G. Klein, N. Hoffman, K. Murray, F. Chang-Diaz, “Interplanetary Propulsion Using Inertial Fusion," LLNL Report UCRL-95275 Rev. 1, 4th Symposium on Space Nuclear Power Systems, Albuquerque, NM, 12-16 Jan. 1987 (1987); C. Orth, G. Klein, J. Sercel, N. Hoffman, K. Murray, F. ChangDiaz, "Transport Vehicle for Manned Mars Missions Powered by Inertial Confinement Fusion," AIAA/SAE/ASME/ ASEE $23^{\text {rd }}$ Joint Propulsion Conference, San Diego, CA, June 29 - July 2, 1987, Paper AIAA-87-1904 (1987); C. Orth, "Interplanetary Space Transport Using Inertial Fusion Propulsion," Ninth International Conference on Emerging Nuclear Energy Systems (ICENES'98), Tel-Aviv, Israel, June 28 July 2, 1, 253-263 (1998).

${ }^{106}$ C. Orth, "Interplanetary Space Transport Using Inertial Fusion Propulsion," Ninth International Conference on Emerging Nuclear Energy Systems (ICENES'98), Tel-Aviv, Israel, June 28 - July 2, 1, 253-263 (1998).

${ }^{107}$ K. A. Murray, “The Gevaltig: An Inertial Fusion Powered Manned Spacecraft Design for Outer Solar System Missions," Report ETEC-89-7 (NASA-CR-185163), Energy Technology Engineering Center, Rocketdyne Division, Rockwell International, Canoga Park, CA (October 1989).

${ }^{108}$ C. Orth, "Interplanetary Space Transport Using Inertial Fusion Propulsion," ibid.

${ }^{109}$ Ralph Moir, LLNL, private communication (1999).

${ }^{110}$ E.g., Marsha Freeman, "Two Days to Mars With Fusion Propulsion," 21 $1^{\text {st }}$ Century Science E Technology, 26 (March-April 1988).

${ }^{111}$ Art Molvik, LLNL magnetic fusion program, LLNL, private communication (1998).

${ }^{112}$ T. J. Nugent, "A Performance Evaluation of the Ion Compressed Antiproton-Catalyzed Nuclear (ICAN) Propulsion System," Jet Propulsion Laboratory Report JPL \#D-8572 (July 1991). 
${ }^{113}$ L. John Perkins, Charles D. Orth, and Max Tabak, “On the Utility of Antiprotons as Drivers for Inertial Confinement Fusion," submitted to Nuclear Fusion (2003).

${ }^{114}$ Bill Wagstaff, "A Spaceship Named Orion," Air \& Space, Smithsonian Institute (Oct./Nov. 1988). 